\title{
Modularity in Belief Change of Description Logic Bases
}

\author{
Ricardo Ferreira Guimarães
}

\author{
THESIS SUBMITTED \\ TO THE \\ Institute of Mathematics And Statistics \\ OF THE \\ University OF SÃO PAUlO \\ IN FULFILLMENT OF THE REQUIREMENTS \\ FOR THE DEGREE \\ $\mathrm{OF}$ \\ Doctor OF SCIENCE
}

Program: Computer Science

Supervisor: Prof ${ }^{\mathrm{a}}$. Dr ${ }^{\mathrm{a}}$. Renata Wassermann

This study was financed in part by the Coordenação de Aperfeiçoamento de Pessoal de Nível Superior - Brasil (CAPES) - Finance Code 001 and by FAPESP through grant 2017/04410-0

São Paulo, December 2019 


\section{Modularity in Belief Change of Description Logic Bases}

This is the original version of the thesis written by the candidate Ricardo Ferreira Guimarães as submitted to the examination committee. 


\section{Acknowledgements}

This thesis would not be the same without the support I had from many people and organisations. First, I would like to thank my supervisor, professor Renata Wassermann. Not only her knowledge aided me considerably, but also her wise and patient nature helped me to remain calm during stressful moments. A good deal of my positive experience in academia is due to her.

In this sense, I also give special thanks for the friends and colleagues that I met at LIAMF. They provided feedback and support when I needed. Indeed, one of the best places I found to discuss subjects related to the $\mathrm{PhD}$, be it a purely academic topic or not.

This work would surely not be the same without my internship at the University of Manchester, where I met professor Uli Sattler who gave me crucial guidance as my supervisor and helped me to see my work in a different light. Further, I would like to extend my thanks to the friends and colleagues that I made there too; professors, students and researchers at the IMG in particular. The discussions, especially at the DL lunch and in the office, proved to be very fruitful. Moreover, they made my experience of living abroad much more rich and pleasant.

For sure, I would not be here without my family. I have no words to thank them enough for all the support they provided me, for believing in my potential and also for the immense effort to make sure I had a good education. They are one of the main reasons I consider myself a very fortunate person.

I also cannot forget to mention the friends that I made through my life, and that stuck around. In particular, I would like to thank those that I meet almost a decade ago when starting my undergraduate course in Computer Science at IME. Even though I have not been very present due to the hardships of the $\mathrm{PhD}$, I still feel very welcome among them.

Also, I acknowledge the support from CAPES and FAPESP for the funding provided. Without the scholarships, I would not have been able to keep studying this far. As such, I also thanks those that, like me, believe that good education (especially if public, accessible for everyone) is a powerful tool for changing society.

Finally, I thank everyone who taught something, notably the professors, which provided me with knowledge and advice on multiple occasions, and that inspired me to follow this path. 


\section{Resumo}

\section{GUIMARÃES, R. F. Modularidade em Revisão de Crenças em Lógicas de Descrição. 2019.}

147 pp. Tese (Doutorado) - Instituto de Matemática e Estatística, Universidade de São Paulo, São Paulo, 2019.

Ontologias escritas em OWL e OWL 2 são uma das ferramentas mais importantes em Representação do Conhecimento atualmente. Elas permitem o compartilhamento de conhecimento de um domínio sem ambiguidade e operar com conhecimento implícito usando motores de inferência. No entanto, as ontologias podem se tornar grandes e muito complexas, dificultando sua manutenção e evolução. Um fator complicador é que uma pequena mudança pode desencadear consequências inesperadas e indesejadas.

Soluções para manutenção correta surgiram paralelarmente em Revisão de Crenças e em Reparo de Ontologias. Apesar de terem visões distintas, as propostas em ambos os campos se baseiam nas Lógicas de Descrição, que sustentam OWL e OWL 2. Portanto, as abordagens de ambos os campos para reparar ontologias são muito semelhantes no nível algorítmico. Consequentemente, ambas as áreas precisam lidar com a alta complexidade do problema de depuração e com o número exponencial de resultados válidos.

Há estudos em Reparo de Ontologias que usam de técnicas de modularisação para extrair subconjuntos menores da ontologia, suficientes para corrigir uma consequência específica. Ainda assim, os efeitos dos módulos no arcabouço de Revisão de Crenças são pouco estudados: tanto os postulados quanto os mecanismos que selecionam o resultado final podem mudar quando um módulo substitui a entrada. Além disso, o impacto no desempenho computacional foi avaliado apenas em corpora pequenos e com pouca variação de parâmetros. E mais, o número de resultados ainda é exponencial e as soluções existentes raramente fornecem meios para mitigar esse problema.

Nesse sentido, esta tese provê uma visão mais clara dos efeitos da modularidade no arcabouço teórico que garante mudanças racionais (consistentes). Também avalia-se empiricamente o impacto da modularidade no desempenho usando módulos baseados em localidade em um cenário mais amplo. Adicionalmente, investiga-se como os módulos podem ajudar os usuários a filtrar e selecionar os melhores resultados com eficiência. Identifica-se uma categoria de módulos para os quais os postulados de Revisão de Crenças permanecem os mesmos e, em condições moderadas, o resultado permanece inalterado. A análise dos dados experimentais mostra que os módulos são benéficos para o desempenho, muitas vezes exibindo ganhos de ordens de magnitude. Além disso, os métodos propostos para auxiliar na seleção de reparos provaram ser competitivos com os métodos existentes.

Palavras-chave: Reparo de Ontologias, Revisão de Crenças, Modularisação de Ontologias. 


\section{Abstract}

GUIMARÃES, R. F. Modularity in Belief Change of Description Logic Bases. 2019. 147 pp. Thesis (PhD) - Institute of Mathematics and Statistics, University of São Paulo, São Paulo, 2019.

Ontologies written in OWL and OWL 2 are one of the most prominent tools in Knowledge Representation nowadays. They allow the sharing of knowledge of a domain unambiguously and operate with implicit knowledge using reasoning algorithms. However, ontologies can become large and very complex, difficulting their maintenance and evolution. One complicating factor is that a small change can trigger unexpected and unwanted consequences.

Solutions to sound maintenance have emerged separately in Belief Change and Ontology Repair. Despite having distinct views, proposals in both fields often rely on the Description Logics, which underpin OWL and OWL 2. Hence, the approaches from both fields for repairing ontologies are very similar at the algorithmic level. Consequently, both areas need to address the high complexity of the debugging problem and cope with the exponential number of correct outcomes.

There are studies in Ontology Repair which use modularity techniques to extract smaller subsets of the ontology which are sufficient to fix a particular consequence. Still, the effect of modules on the Belief Change framework is poorly understood: either the postulates or the mechanisms which select the final result might change when a module replaces the input. Also, the impact on computational performance was only assessed in small corpora and with few variations in parameters. Further, the number of outcomes is still exponential, and existing solutions rarely provide means to mitigate this issue.

In this direction, this thesis provides a clearer understanding of the effects of modularity in the theoretical framework that guarantees rational (sound) changes. Also, it evaluates the performance impact of modularity empirically using locality-based modules in a broader setting. Additionally, it also investigates how modules can aid users to filter and select the best results efficiently. A category of modules is identified for which the postulates from Belief Change remain the same, and under mild conditions, the result is unchanged. The analysis of experimental data shows that modules are beneficial for performance, often displaying gains of orders of magnitude. Further, the methods proposed to aid in the selection of repairs are shown to be competitive with existing methods.

Keywords: Ontology Repair, Belief Change, Ontology Modularisation. 


\section{Contents}

List of Figures $\quad$ xi

List of Tables $\quad$ xiii

1 Introduction $\quad 1$

1.1 Notation . . . . . . . . . . . . . . . . . . . . . . . . . 5

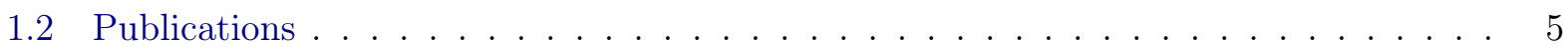

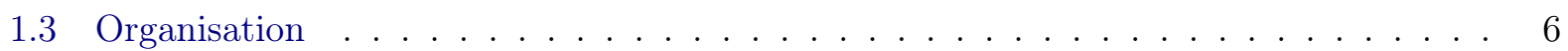

$\begin{array}{lll}2 & \text { Preliminaries } & 7\end{array}$

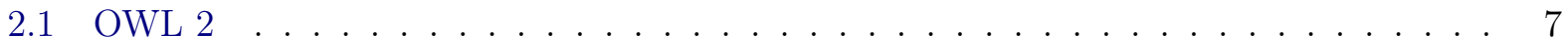

2.2 Description Logics $\ldots \ldots \ldots \ldots \ldots \ldots \ldots \ldots$

2.3 Belief Base Change in Description Logics . . . . . . . . . . . . . . . . . . . 10

2.3 .1 The AGM Paradigm . . . . . . . . . . . . . . . . . . 10

2.3.2 From AGM to Description Logic Belief Bases . . . . . . . . . . . . . . . . 10

2.3.3 Bridging Belief Change and Ontology Repair . . . . . . . . . . . . . . . . 11

2.3.4 Black-box Algorithms for Base Change of DL Bases . . . . . . . . . . . . . 19

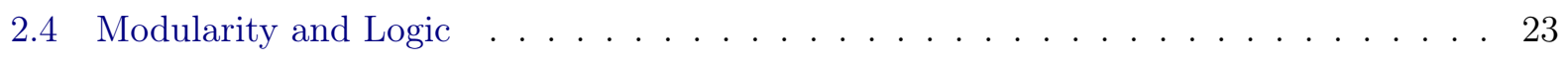

2.4 .1 Local Change . . . . . . . . . . . . . . . . . . . . 25

2.4 .2 Modularity in Ontologies . . . . . . . . . . . . . . . 26

2.4 .3 Syntactic Locality-Based Modules _ . . . . . . . . . . . . . . . . . 27

2.4 .4 Atomic Decomposition . . . . . . . . . . . . . . . . 33

3 Modularity in Ontology Repair $\quad 37$

3.1 State-of-art . . . . . . . . . . . . . . . . . . . 37

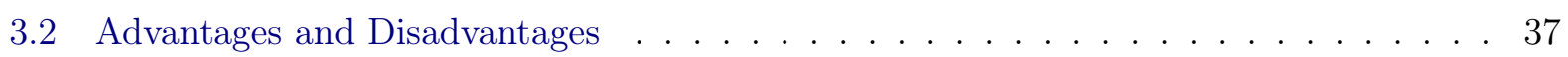

3.2 .1 Arguments in Favour . . . . . . . . . . . . . . . . . . . . . . 38

3.2 .2 Arguments Against . . . . . . . . . . . . . . . . . . . . . . . . . . . . . . . . . . . . . . . .

3.3 Research Agenda . . . . . . . . . . . . . . . . . . . . . . . . . . . . . . . 39

4 Modularity-Aware Belief Base Change in Description Logics 41

4.1 Justification-Preservation in Belief Change of Description Logics Bases . . . . . . . . 41

4.2 Locality-Based Modules Incremental Modularity Bounds . . . . . . . . . . . . . . . 47 
5 Experimental Framework

5.1 Corpus Generator for Belief Change and Ontology Repair . . . . . . . . . . . . . . 53

5.1 Case Specification . . . . . . . . . . . . . . . . . . . . 54

5.1 .2 Instantiation . . . . . . . . . . . . . . . . . 57

5.1 .3 Limitations . . . . . . . . . . . . . . . . . . . . . . . 58

5.2 Measurements . . . . . . . . . . . . . . . . . . . . . 58

5.2 .1 Running Time . . . . . . . . . . . . . . . . . . . . 58

5.2 .2 Memory . . . . . . . . . . . . . . . . . . . . . 60

5.2 .3 Reasoner Calls . . . . . . . . . . . . . . . . . . . . . . . . . 60

5.3 Benchmark Considerations . . . . . . . . . . . . . . . . . . . 60

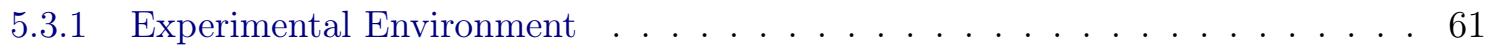

5.4 Other Experimental Parameters . . . . . . . . . . . . . . . . . . . . 63

5.4 .1 Dataset Choice . . . . . . . . . . . . . . . . . . . . . 63

5.4 .2 OWL Reasoner Choice . . . . . . . . . . . . . . . . . . . . . . . . . 64

5.5 Supporting Material . . . . . . . . . . . . . . . . . . . . . 64

$6 \quad$ Empirical Evaluation $\quad 65$

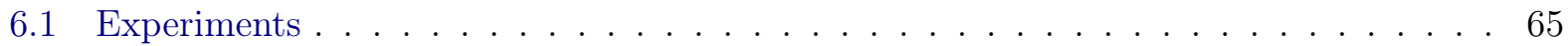

6.2 Results . . . . . . . . . . . . . . . . . . . . . 67

6.2.1 Preliminary Considerations . . . . . . . . . . . . . . . . 68

6.2 .2 Construction of Complete Sets _ . . . . . . . . . . . . . . . 68

6.2 .3 Construction of Single Solutions _ . . . . . . . . . . . . . . . . . . . 83

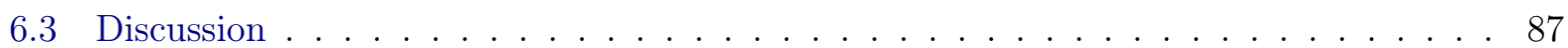

7 Modularity-based Stratification $\quad 103$

7.1 Stratification for Belief Change in Description Logics . . . . . . . . . . . . . . . . 103

7.2 Stratification Methods . . . . . . . . . . . . . . . . . 104

7.2 .1 Axiom Specificity . . . . . . . . . . . . . . . . . . . . . . 104

7.2 .2 Concept Name Specificity . . . . . . . . . . . . . . . . 107

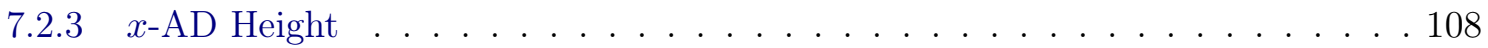

7.3 Computing Stratified Constructions . . . . . . . . . . . . . . . 109

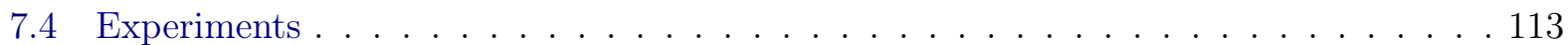

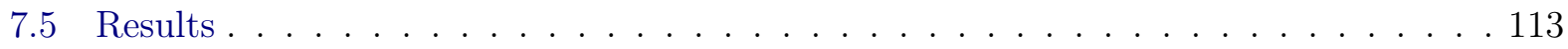

7.5.1 Axiom Dispersion . . . . . . . . . . . . . . . . . . . . 113

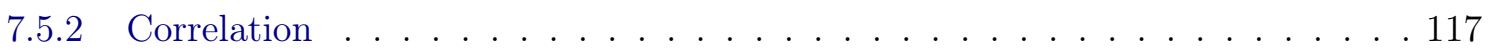

7.5 .3 Filtering on Outcomes . . . . . . . . . . . . . . . . . . 118

7.5 .4 Discussion . . . . . . . . . . . . . . . . . . . . . . . . . . . 119

8 Conclusion $\quad 121$

8.1 Future Works . . . . . . . . . . . . . . . . . . . . . . . . . . . . . . . . . . . . .

$\begin{array}{ll}\text { Bibliography } & 125\end{array}$ 


\section{List of Figures}

1.1 Illustrative ontology MovieEx . . . . . . . . . . . . . . . . . . . . 1

2.1 Relationship between concepts in Belief Change and Ontology Repair [MGSW19] . . 12

2.2 Syntactic locality conditions . . . . . . . . . . . . . . . . . . 28

$2.3 T \perp^{*}$-AD of the example ontology AccountEx . . . . . . . . . . . . 34

6.1 Distribution of inputs according to ontology size and input signature size . . . . . . 69

6.2 Frequency histogram of input cases according to relative module size . . . . . . . . 70

6.3 Distribution of input according to MinImps size and average number of axioms per MinImp . . . . . . . . . . . . . . . . . . . 71

6.4 Distribution of input according to MaxNons size and average number of axioms per

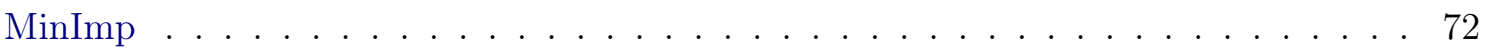

6.5 Distribution of $\log 2 \mathrm{FC}$ for reasoner calls spent in average by Basic-MI and SME-MI in Full-M2 . . . . . . . . . . . . . . . . . . . . . . . . 73

6.6 Distribution of $\log 2 \mathrm{FC}$ for reasoner calls spent in average by Basic-MN and SME-MN in Full-M2 . . . . . . . . . . . . . . . . . . . . . . . . . . 74

6.7 Distribution of $\log 2 \mathrm{FC}$ for reasoner calls spent in average by Basic-MI and Basic-MN in Full-M2 . . . . . . . . . . . . . . . . . . . . . . . . 74

6.8 Distribution of $\log 2 \mathrm{FC}$ for reasoner calls spent in average by SME-MI and SME-MN in Full-M2 . . . . . . . . . . . . . . . . . . . . . . . . 75

6.9 Distribution of $\log 2 \mathrm{FC}$ for CPU time spent in average by Basic-MI and SME-MI in

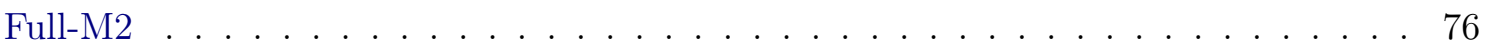

6.10 Distribution of $\log 2 \mathrm{FC}$ for CPU time spent in average by Basic-MN and SME-MN in Full-M2 . . . . . . . . . . . . . . . . . . . . . . . 77

6.11 Distribution of $\log 2 \mathrm{FC}$ for CPU time spent in average by Basic-MI and Basic-MN in

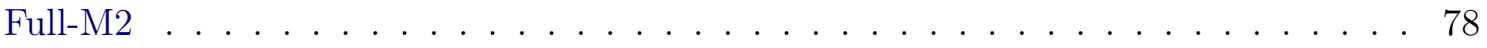

6.12 Distribution of log2FC for CPU time spent in average by SME-MI and SME-MN in

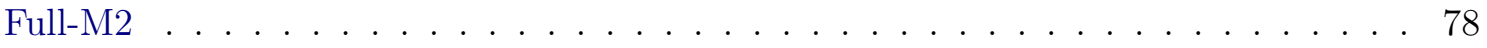

6.13 Distribution of maximum memory (VmHWM) consumed in average by Basic-MI and

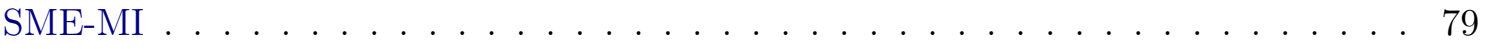

6.14 Distribution of maximum memory (VmHWM) consumed in average by Basic-MN and $\mathrm{SME}-\mathrm{MN} \ldots \ldots \ldots \ldots$

6.15 Distribution of maximum memory (VmHWM) consumed in average by Basic-MI and Basic-MN in Full-M2 . . . . . . . . . . . . . . . . . . . . . . . 90 
6.16 Distribution of maximum memory (VmHWM) consumed in average by SME-MI and SME-MN in Full-M2 . . . . . . . . . . . . . . . . . . . . . . . . 90

6.17 Distribution of $\log 2 \mathrm{FC}$ for reasoner calls spent in average by Basic-MI and MU-MI in Single-M2 . . . . . . . . . . . . . . . . . . . . . . . . . 91

6.18 Distribution of log2FC for reasoner calls spent in average by Basic-MN and SME-MN in Single-M2 . . . . . . . . . . . . . . . . . . . . . . . . 92

6.19 Distribution of log2FC for reasoner calls spent in average by Basic-MI and Basic-MN in Single-M2 . . . . . . . . . . . . . . . . . . . . . . . . 93

6.20 Distribution of $\log 2 \mathrm{FC}$ for reasoner calls spent in average by MU-MI and SME-MN in Single-M2 . . . . . . . . . . . . . . . . . . . . . . . . 94

6.21 Distribution of $\log 2 \mathrm{FC}$ for CPU time spent in average by Basic-MI and MU-MI in

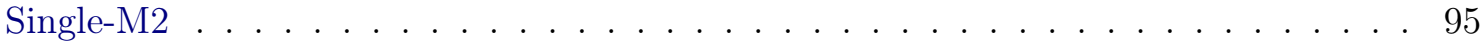

6.22 Distribution of $\log 2 \mathrm{FC}$ for CPU time spent in average by Basic-MN and SME-MN in Single-M2 . . . . . . . . . . . . . . . . . . . . . 96

6.23 Distribution of $\log 2 \mathrm{FC}$ for CPU time spent in average by Basic-MI and Basic-MN in

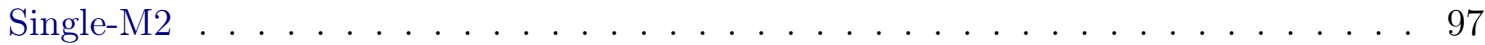

6.24 Distribution of $\log 2 \mathrm{FC}$ for CPU time spent in average by MU-MI and SME-MN in

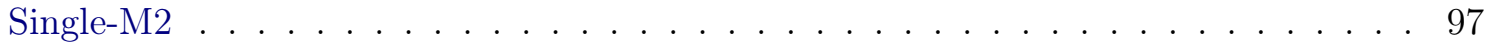

6.25 Distribution of maximum memory (VmHWM) consumed in average by Basic-MI and MU-MI in Single-M2 . . . . . . . . . . . . . . . . . . . . . . . . 98

6.26 Distribution of maximum memory (VmHWM) consumed in average by Basic-MN and SME-MN in Single-M2 f . . . . . . . . . . . . . . . . . . . 99

6.27 Distribution of maximum memory (VmHWM) consumed in average by Basic-MI and Basic-MN in Single-M2 . . . . . . . . . . . . . . . . . . . . 100

6.28 Distribution of maximum memory (VmHWM) consumed in average by MU-MI and SME-MN in Single-M2 . . . . . . . . . . . . . . . . . . . . . . . 101

7.1 Atomic decompositions and inferred concept hierarchy of the KoalaS ontology (dashed lines illustrate possible stratifications $) \ldots \ldots$. . . . . . . . . . . . . . . . . . . . . .

7.2 Cumulative density histograms of stratum relative sizes over the corpus. . . . . . . 115

7.3 Distribution of the indistinguishable axioms rates . . . . . . . . . . . . 116

7.4 Distribution of correlation between the methods (for p-value $<0.05$ ) . . . . . . . 117

7.5 Scatter plot relating the number of best MaxNons according to AxS and the original size . . . . . . . . . . . . . . . . . . . . . . . . . 119

7.6 Scatter plot relating the number of best MaxNons according to BAH and the original size . . . . . . . . . . . . . . . . . . . . . . . . . 119 


\section{List of Tables}

2.1 Complex concepts in $\mathcal{S R O I} \mathcal{Q} \ldots \ldots \ldots \ldots \ldots \ldots \ldots$

2.2 Axioms in $\mathcal{S R O I} \mathcal{O} \ldots \ldots \ldots \ldots \ldots \ldots \ldots \ldots$

5.1 Experimental machines' specification . . . . . . . . . . . . . . . . . . 62

6.1 Parameters of each sub-experiment for research question Q2 $\ldots \ldots 6$

6.2 Statistics of the coefficient of variation in each combination of parameters, for each experiment and each quantity measured f . . . . . . . . . . . . 68

6.3 Number of considered ontologies per OWL 2 profile (note that each category excludes the others, e.g. EL+RL means that the ontology is in OWL 2 EL and OWL 2 RL but not in OWL 2 QL). . . . . . . . . . . . . . . . . 70

6.4 Statistics of the $\log 2 \mathrm{FC}$ from SME-MI to MU-MI Full-M1 and Full-M2 . . . . . . . 70

6.5 Summarised statistics of reasoner calls $\log 2 \mathrm{FC}$ per method pair in Full-M1 and Full-M2 75

6.6 Summarised statistics of processing time log2FC per method pair in Full-M1 and

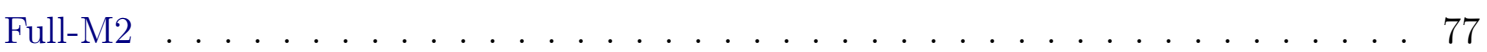

6.7 Number of group of executions per timeout status and method (2224 groups) in Full-M1 80

6.8 Number of group of executions per timeout status and method (3328 groups) in Full-M2 80

6.9 Number and percentage of executions per method that achieve the smallest (best) value in each quantity within a margin of $5 \%$ from the actual minimum in Full-M1 . 81

6.10 Number and percentage of executions per method that achieve the smallest (best) value in each quantity within a margin of $5 \%$ from the actual minimum in Full-M2 . 81

6.11 Correlations between the fold changes and ontology size, value of the quantities measured and the relative size of the module for Full-M1 (largest p-value: $1.7999 \times 10^{-5}$ ) 82

6.12 Correlations between the fold changes and ontology size, value of the quantities measured and the relative size of the module for Full-M2 (largest p-value: $5.0993 \times 10^{-3}$ ) 82

6.13 Statistics of the log2FC from Queue to Stack for each method in Single-M2 . . . . . 83

6.14 Statistics of the $\log 2 \mathrm{FC}$ from SME-MI to MU-MI in Full-S2 . . . . . . . . . . . . 84

6.15 Number of group of executions per timeout status and method (2224 groups) in

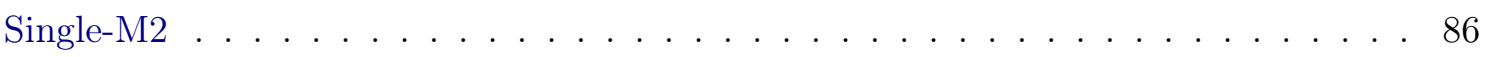

6.16 Number and percentage of executions per method that achieve the smallest (best) value in each quantity within a margin of $5 \%$ from the actual minimum in Full-S2 . . 86

6.17 Correlations between $\log 2 \mathrm{FC}$ between methods using queue as HST structure and ontology size, average value of the quantities measured and the relative size of the module for Single-M2 (values in grey had p-value $>0.01) \ldots . . \ldots$. . . . . 87 
6.18 Correlations between the fold changes and ontology size, average value of the quantities measured and the relative size of the module for Single-M2 (values in grey had

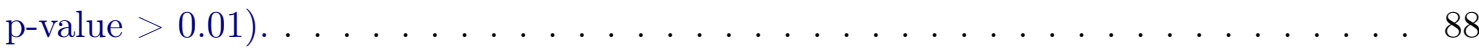

7.1 Mapping of OWL 2 DL logical axioms to axiom specificity categories . . . . . . . 105

7.2 Conversion of some OWL 2 DL axioms to concept inclusions . . . . . . . . . . . . 106

7.3 The KoalaS ontology and the rankings of its axioms according to the five methods considered . . . . . . . . . . . . . . . . . . . . . . . . 109

7.4 Specification of machine 3 (M3) employed in the stratification comparison experiments115

7.5 Corpus correlation values between stratification methods (largest p-value: $2.8208 \times$

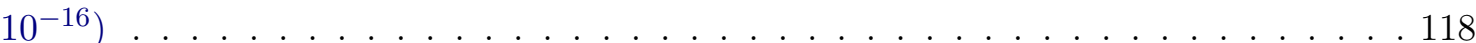




\section{Chapter 1}

\section{Introduction}

Having clear definitions is perhaps one of the first steps towards devising reliable, intelligent systems. In this sense, Artificial Intelligence and Knowledge Representation can provide tools to develop such agents, in particular via approaches which can formalise these definitions unambiguously. Studies in these (and other) areas led to the creation of the ontologies, which are nowadays one of the most prominent tools in formalising the knowledge of a group about a domain. They allow users to define concepts and relationships between them in a precise way that can be shared and understood.

Although ontologies can be defined, and worked with, in a variety of ways one of the most common definitions states that an ontology is a formal representation of accorded knowledge about a domain, made to be shared and also written in a computer accessible language. This definition is an extension of Gruber's [Gru93].

Figure 1.1 illustrates what type of knowledge ontologies commonly represent. Each node corresponds to a concept, they can be atomic (drawn with full lines) or constructed with operators (those with dashed borders). Each arrow corresponds to an axiom, a relationship between concepts. Note that the graphical notation is used only for informative purposes and it is not bound to any established standard.

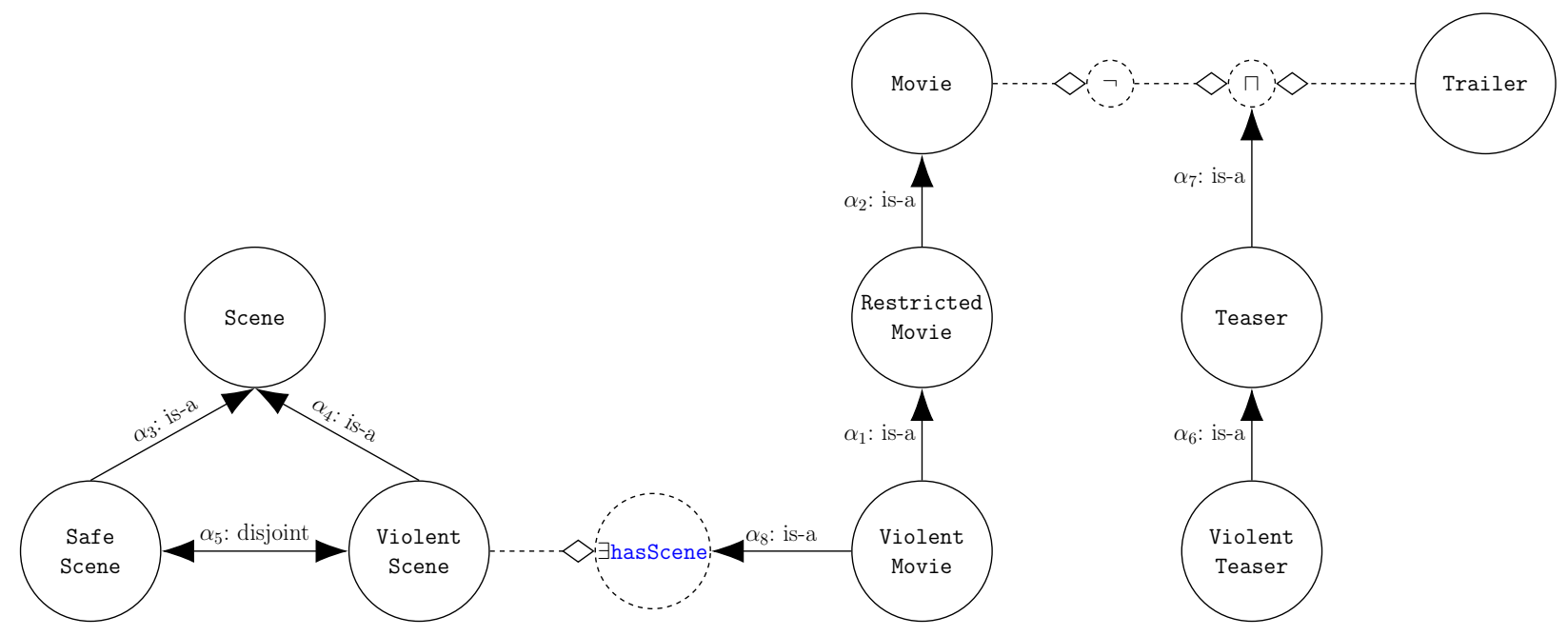

Figure 1.1: Illustrative ontology MovieEx

Ontology repositories such as Ontohub ${ }^{1}$ and NCBO BioPortal ${ }^{2}$, large scale ontologies (e.g. SNOMED $\mathrm{CT}^{3}$, the Gene Ontology $\left.(\mathrm{GO})^{4}\right)$ and the growth of the studies in this area attest for their success. Today, applications using ontology enjoy benefits of representation of concepts with

\footnotetext{
${ }^{1}$ https://ontohub.org/

${ }^{2}$ https://bioportal.bioontology.org/

${ }^{3}$ http://www.snomed.org/

${ }^{4}$ http://geneontology.org/
} 
a formal semantics which facilitates collaboration across different groups, both in academia and in the industry.

Part of this progress is due to the need for guarantees that several people are using the same definitions. As remarked by Matentzoglu [Mat16], this is particularly true in areas rich in assertional knowledge such as Biology and Medicine. Another decisive factor is the creation of a standard language, more specifically the W3C recommendation of OWL, and more recently OWL 2 , as the ontological languages for their Semantic Web stack.

Large portions of both OWL and OWL 2 are founded on the family of the Description Logics (DLs), namely the profiles OWL DL and OWL 2 DL, respectively. These logics are decidable fragments of First Order Logic (FOL), this allows the development of programs, called reasoners, which extract implicit information from the formulas expressed in this logic. The features of this family of logics allow reasoners to be sound, complete and terminating procedures.

It is possible to map any DL ontologies, i.e. those in OWL DL or OWL 2 DL, to a set of logical formulas (or axioms) in some DL. This mapping provides users with the ability to query the ontology for information that, albeit not expressed explicitly, entails from the formalisation at hand. For instance, consider the example of Figure 1.1, the information that a ViolentMovie is a Movie is implicit: it is a consequence of the relationships expressed.

Ontologies have, traditionally, two parts, the TBox which holds terminological knowledge (the relationships between concepts). The ABox corresponds to assertional information (information about individuals). Figure 1.1 denotes only terminological knowledge (empty ABox), however, if one includes information about a specific individual, that is a particular Movie, or Scene, or any other entity, that knowledge is held in the ABox. Such differentiation facilitates comprehension, although both parts are mapped to formulas in DLs. Baader et al. [BHLS17] compare the TBox with a database schema and the ABox to the set of instances.

The ability to operate with tacit knowledge is one of the most powerful features of this technology. However, this capacity comes with a cost: deciding entailment in $\mathcal{S} \mathcal{R O} \mathcal{I} \mathcal{Q}(\mathcal{D})$, the DL underpinning OWL 2 DL, is a problem in N2ExPTime [Kaz08]. The high complexity of reasoning often makes it prohibitive for many use-cases.

Even with this restriction, the field progresses to circumvent this problem. Further, there are lightweight DLs, such as $\mathcal{E} \mathcal{L}++$, whose reasoning problem is in PTime [BBL05], and that is sufficient to express the knowledge in well-known ontologies such as SNOMED CT. There are many Description Logics other than $\mathcal{E} \mathcal{L}$ and $\mathcal{S} \mathcal{R O} \mathcal{I} \mathcal{Q}(\mathcal{D})$, each in a different point tradeoff between expressivity and reasoning complexity. Further, even for complex ontologies some reasoning tasks do not require the worst case complexity.

Besides the complexity and costs of reasoning per se, there are still other obstacles to the adoption of ontologies. One of them is that ontologies, principally the larger and complex ones, are difficult to maintain. As a consequence of being able to provide implied relationships between concepts via logical inference, even a simple modification might introduce changes unforeseen to the user or designer. Ontology editors such as Protégé ${ }^{5}$ have some support for debugging and repairing ontologies (in Protégé's case via the plugins OntoDebug ${ }^{6}$ and OWLExplanation ${ }^{7}$ ), but there are still shortcomings concerning efficiency and aid in the decision process.

For instance, adding a new piece of knowledge that contradicts some previous (explicit or implicit) information may turn the whole ontology inconsistency. Inconsistencies render all consequences meaningless, as they make the ontology entail everything. Another common example of faulty behaviour is incoherence: when one term in the ontology can only assume empty interpretations.

Inconsistencies and incoherences are not the only types of error in ontologies. A famous example of faulty behaviour that is neither incoherence nor inconsistency is that a version of the SNOMED $\mathrm{CT}$ entailed that the amputation of a finger implied in the amputation of the whole hand, even

\footnotetext{
${ }^{5}$ https://protege.stanford.edu/

${ }^{6}$ http://isbi.aau.at/ontodebug/

${ }^{7}$ http://owl.cs.manchester.ac.uk/research/explanation/
} 
though the formalisation was logically consistent. After identifying an erroneous consequence like that, pinpointing its causes is an NP-hard problem even for lightweight DLs [BPS07, Peñ09]. Further, after determining the problem's source, there is still a repair to be made.

There are two notable fields in Knowledge Representation whose topics include debugging and repairing ontologies. One of them is the aptly named Ontology Repair; the other is called Belief Change. The first studies how to debug and fix ontologies, usually relying on the logical background provided by the Description Logics. The field of Belief Change [FH11] addresses the broader problem of rational change of beliefs performed by intelligent agents, although more general, it has been adapted to ontologies by managing them as belief bases of DL formulas [Han91, Han94b, Flo06, Rib13].

The two fields have addressed the same problem with different views. Ontology Repair being more pragmatic and closer to the ontologies and Description Logics, while Belief Change solutions tend to use a foundational approach sustained by logical postulates and characterisations which have survived the test of time with small modifications.

Research in Ontology Repair often focus on single description logic (e.g. $\mathcal{E} \mathcal{L}, \mathcal{A L C}$ ) and almost always try to perform a repair by looking at the minimal subsets of the ontology that cause the undesired consequence (known as justifications [Kal06, Hor11], among other names). Further, most of these studies search only for maximal repairs, in a set-inclusion sense. Also, in this area, the methods proposed often explicit the differences when repairing the TBox or the ABox [DQ15, BBPW17]. Many also use the hierarchy of concepts, and other features specific to the ontologies [QLB06].

Belief Change historically focused on computing the maximal subsets of the ontology that do not imply a certain entailment. This field began with the AGM theory [AGM85], which only worked for classical logics and for sets that needed to be inference-closed. Fortunately, authors such as Hansson, Wassermann, Flouris and Ribeiro [Han91, HW02, Flo06, Rib13] adapted this other formalisms and representations, for instance, sets of DL formulas which are not (necessarily) closed under consequence, that is, DL ontologies.

Some aspects of the traditional theory remain such as the definition of operations which represent the possible ways an agent might change its beliefs, the presentation of postulates to rule them, and the mapping of the postulates to mathematical constructions. In this field, the two main constructions are defined with minimal implying (faulty) subsets or maximal subsets that are correct. The postulates do not determine a single function as possible and the maximality of a repair is not a constant requirement.

Thus, in Belief Change ontology repair is mapped to the problem of changing a set of formulas in some DL according to one the operations defined, using only general properties of these logics such as monotonicity and compactness.

Even with views primarily distinct, similar proposals to ontology maintenance emerged in both fields. These solutions involve either finding the minimal subsets responsible by the undesired entailments or finding the maximal subset of the ontology which does not display the faulty behaviour. It is important to remark that repair algorithms in both areas appeared using either minimal implying subsets or maximal non-implying subsets though few studies connect the advancements in both fields.

The solutions, however, still suffer from the complexity of the debugging problem. Both the number of minimal implicants and that maximally correct subsets can be exponential on the number of axioms of an ontology [BPS07]. Consequently, there can be an exponential number of repairs on the number of formulas expressed. Further, most of the alternatives provided to aid a user in deciding the best outcome, out of the correct ones, assume that an importance ordering of the formulas (or axioms) given. However, such an order is hardly available beforehand.

Therefore, the algorithms for maintenance of these knowledge bases still need to be improved to allow more systems to enjoy the semantic capabilities accrued by ontologies. Otherwise, both the cognitive and computational costs will hamper their usage in applications which would be more reliable and well-understood. Ontologies and reasoning are particularly interesting in tasks which need explainable conclusions: the reasoning itself can provide the logical proofs which underpin the 
entailments given, also, to allow the user to query the model represented.

To handle large ontologies, with thousands of axioms (as is the case of SNOMED CT) it is possible to extract portions of them that are sufficient for a specific task. These subsets are called modules, and the whole area of Ontology Modularisation focuses on developing new types of modules and studying their properties. For instance, for the example of Figure 1.1, a module could omit information about scenes whenever the application is only interested in trailers.

In this direction, this thesis investigates the effectiveness of modularity on addressing the shortcomings of current approaches to debug and fix ontologies. Namely, these issues correspond to the high computational costs of repairing ontologies and the lack of tools aiding the decision process. As mentioned earlier, modules are (in a rough sense) subsets of the ontology that suffice for a specific task. Modularity approaches have been used on reasoning and even on pinpointing causes for undesired consequences [SQJH08, Hor11, Mat16].

There are approaches in the theory of Belief Change that propose similar improvements as those provide by modularity. Still, the types of modules suitable for Belief Change and their impact on the theoretical framework are open questions.

Although the usage of modules in Ontology Repair is not novel, the actual impact in terms of computational performance is still not well documented. Most studies restrict themselves to very few ontologies or do not measure the effect of modularity alone. Further, most of the studies only use modules with minimal implying subsets. Hence, the impact on approaches which use the maximally correct subsets is still unknown.

Additionally, both in Belief Change and Ontology Repair, there are methods to diminish the number of choices that a user has to make among the set of possible repairs. Often the solution is to assume the existence of partial order between the axioms and use it to filter the best outcomes. What is missing from these proposals is how to obtain these orderings.

The objective of this thesis is to provide a clearer understanding of the impact of modularity in Ontology Repair and Belief Change methods. In particular, it extends previous studies in this subject with theoretical and empirical results. The first part consists of putting modules into the framework of Belief Change. The second is to assess the impact of modularity on algorithms in both fields using a broader experiment. The third corresponds to the proposal and evaluation of modularity-inspired orderings to mitigate the choice problem.

There are two main challenges when linking modules and the theory of Belief Change (more specifically its version dedicated to DL ontologies). One is the characterisation of suitable approaches, which preserves the postulates as much as possible. The other is to identify whether the result changes with modularity, and if so, how to recover the original.

Before proceeding to the theoretical results, some effort is spent on closing the gap between Ontology Repair and Belief Change. This bridging attempt aids the presentation of the results to readers familiar with either field, which is also a minor contribution and continuation of previous work.

As a theoretical contribution, a category of modules that guarantees seamless integration into the operations defined in Belief Change is identified, and under mild conditions, the outcome is unaltered after modularisation. Following the modifications in the theoretical framework, the algorithms are also revisited to assess their compatibility.

Concerning the empirical evaluation of the impact of modularity, the experiment design aims to broaden the results of existing research and measure the effect of modules in terms of the number of reasoner calls, computational time spent and the peak of memory consumed.

Before the experiments mentioned, there is also a minor contribution which is the preparation of the actual corpus. Currently, there is no corpus for ontology repair and the solutions provided in the literature are ad hoc. Thus, a prototype generator is built, receiving two datasets: an ontology and a set of template issues. The output is a set of erroneous ontologies to be repaired together with supporting files.

Besides increasing the size of the dataset used to measure effects of modules, the experiment also varies reasoners and runtime environments. Furthermore, measures were taken to reduce the noise 
in the executions, guaranteeing the stability of the results. Based on the literature, the expectation is that modules have an impact of "orders of magnitude" [Hor11] in all quantities considered. The results show that this expectation is not always fulfilled, but that modules are still worthwhile, particularly in the most costly situations.

The results also highlight the similarities and differences of performance between two standard constructions, via minimal implying subsets, and maximally correct subsets. Moreover, a smaller experiment also attests that using depth-first search and depth-first search is not likely to produce significant changes.

The contributions of this thesis finish with the proposal and evaluation of the modularity-based stratification methods. These methods aim to mitigate the multiple outcome problem giving an ordering of the axioms that induces a ranking on the possible repairs. The results are positive, indicating that such strategies are competitive considering existing alternatives.

\subsection{Notation}

In this thesis, the symbol $\mathfrak{L}$ represents a generic logic (or language). $\mathrm{Cn}_{\mathfrak{L}}$ is the consequence operator of that logic which is assumed to be Tarskian (satisfy monotonicity, inclusion and idempotence), compact and satisfy the deduction theorem. The subscript $\mathfrak{L}$ in $\mathrm{Cn}_{\mathfrak{L}}$ is omitted whenever the particular logic $\mathfrak{L}$ is irrelevant or clear from context. Lowercase Greek letters indicate single formulas (or axioms) and uppercase Latin sets of formulas. For some sets of formulas with special meanings, other symbols will be used; in particular, calligraphic letters will denote input ontologies and the symbols $\Omega$ and $\Phi$ will also have a special role (discussed in Chapter 2). Also, due to historical reasons, the letter $\Sigma$ refers to set of terms (signatures).

If $X$ is a set, $\mathcal{P}(X)$ is the power set of $X$ and $\mathcal{P}_{\text {fin }}(X)=\{P \in \mathcal{P}(X) \mid P$ is finite $\}$. Further, the symbol $\amalg$ will represent the disjoint union of sets.

Additional notation concerning Belief Change, modules, and Description Logics and OWL will be introduced in specific sessions in Chapter 2. Other symbols will be presented on a case-by-case basis.

\subsection{Publications}

This thesis includes portions of studies presented as papers in international workshops and in parts of books:

- Ricardo Guimarães and Renata Wassermann. Local change in ontologies with atomic decomposition. In Proceedings of the 3rd Joint Ontology Workshops (JOWO 2017), Bozen-Bolzano, Italy, September 21-23, 2017, volume 2050 of CEUR Workshop Proceedings. CEUR-WS.org, 2017. 87, 92. Preliminary observations that now are part of Chapter 7.

- Ricardo Guimarães, Uli Sattler, and Renata Wassermann. Ontology stratification methods: A comparative study. In Kristijonas Cyras, Tiago Oliveira, Matthew Williams, Loris Bozzato, Martin Homola, Till Mossakowski, and Antoine Zimmer- mann, editors, Proceedings of the Joint Proceedings of Reasoning with Ambiguous and Conflicting Evidence and Recommendations in Medicine (MedRACER 2018) and the 3rd International Workshop on Ontology Modularity, Contextuality, and Evolution (WOMoCoE 2018) co-located with the 16th International Conference on Principles of Knowledge Representation and Reasoning (KR 2018), Tempe, Arizona, USA, Octo- ber 29th , 2018., volume 2237 of CEUR Workshop Proceedings, pages 51-62. CEUR- WS.org, 2018. 87, 98 [GSW18]. An earlier version of Chapter 7.

- Vinícius Bitencourt Matos, Ricardo Ferreira Guimarães, Yuri David Santos, and Renata Wassermann. Pseudo-contractions as gentle repairs. In Carsten Lutz, Uli Sattler, Cesare Tinelli, Anni-Yasmin Turhan, and Frank Wolter, editors, Description Logic, Theory Combination, and All That - Essays Dedicated to Franz Baader on the Occasion of His 60th 
Birthday, volume 11560 of Lecture Notes in Computer Science, pages 385-403. Springer, 2019 [MGSW19]. Subsection 2.3.3 extends on the notation proposed and builds on bringing Ontology Repair and Belief Change together.

\subsection{Organisation}

Chapter 2 contains the preliminaries need to define the research problem (and the solutions proposed). In particular, it presents briefly OWL 2 and Description Logics, gives an overview of the theory of Belief Change with a focus on its adaptation to DL ontologies and a discusses modularity approaches based in Logic. This chapter introduces the modularity techniques that this thesis focus on: the syntactic locality-based modules (LBMs) [CHKS08] and the atomic decomposition (AD) [DPSS11]. It also contains a minor contribution consisting of bridging Ontology Repair and Belief Change concepts via new notation and secondary observations.

Next, Chapter 3 focuses on the state-of-art of modularity in Ontology Repair. It briefly describes the current panorama and discusses the pros and cons of using modules. Further, it also defines the research questions that guide the remaining of the study.

Chapters 4 to 6 focus on the effect of modules to solve the challenge of computational costs. Chapter 4 concentrates most of the theoretical efforts on linking modules and the Belief Change framework. While most of the results apply to a broad category of modularisation approaches, there are remarkable results and algorithms tailored to syntactic LBMs.

After that comes the empirical part. Chapter 5 presents the experimental framework used to assess the impact in practice, including a description and justification of the datasets and corpus generator, reasoners and the methodology adopted. Then, Chapter 6 discusses the results obtained with the experiments, assessing the differences in resources consumed after modularisation. The two standard repair strategies are compared too. Further, it verifies the hypotheses that explain the gains via correlation tests.

Chapter 7 is dedicated to the second challenge, that, is the choice problem. It has both the definitions of the methods and empirical evaluation, although in a smaller scale than the experiments of Chapter 6.

Finally, Chapter 8 contains the summary of contributions, closing remarks and also highlights the next steps envisioned as continuation of this thesis. 


\section{Chapter 2}

\section{Preliminaries}

This chapter establishes the fundamental concepts needed to understand the contributions made. It also presents notation used for Description Logic formulas and Belief Change operations. Further, it describes the adaptation Belief Change's results from propositional logic to Description Logics and relates the theory proposed with concepts in Ontology Repair. Finally, it introduces the theme of Modules and Logic and the approaches in this area that this thesis employs.

\section{$2.1 \quad$ OWL 2}

The ontological language of most relevance to this work is OWL 2, which is endorsed by the $\mathrm{W}_{3} \mathrm{C}^{1}$ as the official language for ontologies on the Semantic Web. There are two variants OWL 2: OWL 2 Full, which undecidable and has its semantics based on RDF graphs and OWL 2 DL which can be translated into the DL $\mathcal{S R O \mathcal { I }}(\mathcal{D})$. OWL 2 DL has also profiles, subsets of the language designed for specific applications: OWL 2 RL (based on Description Logic Programs, DLPs), OWL 2 QL (based on DL-Lite) and OWL 2 EL (based on the language $\mathcal{E L}++$ ) $\left[\mathrm{HKP}^{+} 09\right]$. Each profile has different expressiveness and computational characteristics. Even so, they are not disjoint: an ontology might belong to more than one profile at the same time.

An OWL 2 ontology consists basically of a document which might include references to other ontologies (imports) and a set of axioms written using the language's constructs and terms defined by the user. For reasoning purposes, the ontologies imported are considered part of the ontology. The terms can be either individuals (elements of the ontologies'domain), classes (similar to those in object-oriented programming) or object properties (represent relations between individuals). There are also additional elements such as annotations which do not affect reasoning.

The next section introduces the Description Logics which underpin OWL DL and OWL 2 DL, in particular, $\mathcal{S} \mathcal{R O} \mathcal{I} \mathcal{Q}(\mathcal{D})$, the most expressive DL this thesis considers.

\subsection{Description Logics}

The term Description Logics (DLs) refers to a class of logics used in Knowledge Representation which replaced frame systems and semantic networks. In general, these languages are decidable fragments of First Order Logic (FOL), although there are variants which do not satisfy these constraints [BHLS17]. All the DLs in this thesis are regarded as decidable fragments of FOL that are at most as expressive as $\mathcal{S} \mathcal{R O} \mathcal{I} \mathcal{Q}(\mathcal{D})$. Hence, satisfying monotonicity and compactness.

When describing the knowledge of a domain in a Description Logic, the terms in the signature are partitioned in three sets:

- Individual names $\left(N_{I}\right)$ : elements of the domain, corresponding to FOL's constants.

\footnotetext{
${ }^{1}$ www.w3c.org
} 
- Concept names $\left(N_{C}\right)$ : represent subsets of the domain, corresponding to unary predicates in FOL.

- Role names $\left(N_{R}\right)$ : determine sets of pairs of elements of the domain (subsets of $\Delta^{2}$ ), corresponding to binary FOL predicates.

Following the notation employed by Del Vescovo [Del13], concept names are represented by symbols such as A, B; arbitrary role names by: $\mathrm{r}, \mathrm{s}$ and individuals by $a, b$. Any subset of the vocabulary is called a signature. The signature of an axiom $\alpha$, denoted by $\operatorname{sig}(\alpha)$, corresponds to terms of the vocabulary used to write $\alpha$. Also, if $X$ is a set of axioms (or formulas), the signature of $X$ is defined as: $\operatorname{sig}(X)=\bigcup_{\alpha \in X} \operatorname{sig}(\alpha)$.

The semantics is given by an interpretation $\mathcal{I}=\left(\Delta^{\mathcal{I}},{ }^{\mathcal{I}}\right)$, where $\Delta^{\mathcal{I}} \neq \emptyset$ is the interpretation domain and ${ }^{\mathcal{I}}$ the interpretation function which maps:

- Each individual $a$ to an element $a^{\mathcal{I}} \in \Delta^{\mathcal{I}}$.

- Each concept name $\mathrm{A}$ to a subset $\mathrm{A}^{\mathcal{I}} \subseteq \Delta^{\mathcal{I}}$.

- Each role name $r$ to a subset $r^{\mathcal{I}} \subseteq \Delta^{\mathcal{I}} \times \Delta^{\mathcal{I}}$.

In the same way as Del Vescovo [Del13], this thesis assumes that the domain of all interpretation functions is precisely the vocabulary of interest (terms outside the vocabulary have undefined interpretation). With this requirement, we can define the concepts below, which will appear later in section.

- Given an interpretation $\mathcal{I}$ and with domain signature $\Sigma_{\mathcal{I}}$, and an arbitrary signature $\Sigma \subseteq \Sigma_{\mathcal{I}}$, the $\Sigma$-projection $\left.\mathcal{I}\right|_{\Sigma}$ of $\mathcal{I}$ is the interpretation such that $\mathrm{t}^{\left.\mathcal{I}\right|_{\Sigma}}=\mathrm{t}^{\mathcal{I}}$ for each term $\mathrm{t} \in \Sigma$. The function $\left.\cdot{ }^{\mathcal{I}}\right|_{\Sigma}$ is undefined for terms not in $\Sigma$.

- Two interpretations $\mathcal{I}$ and $\mathcal{J}$ coincide on a signature $\Sigma$, if $\Delta^{\mathcal{I}}=\Delta^{\mathcal{J}}$ and $\mathrm{t}^{\mathcal{I}}=\mathrm{t}^{\mathcal{J}}$ for all $X \in \Sigma$. In symbols: $\left.\mathcal{I}\right|_{\Sigma}=\left.\mathcal{J}\right|_{\Sigma}$.

Each description logic provides two sets of operators. One of these sets correspond to the constructors used to combine the terms of the signature into complex concepts or roles, the other has the symbols used to write actual formulas, expressing relationship between the atomic (parts of the signature) or constructed entities. These sets determine the expressivity of the logic [BHLS17].

Since this thesis focuses on OWL 2 DL ontologies, $\mathcal{S R O I Q}(\mathcal{D})$ will be the most expressive description logic considered. The syntax and semantics of this language are describe next.

First, a $\mathcal{S R O I} \mathcal{Q}$ concept is either simple or complex. The simple concepts are those in the signature $\left(N_{C}\right)$ or those with predetermined definitions: $T$ (whose interpretation in the whole domain) and $\perp$ (whose interpretation is the emptyset). Table 2.1 describes the complex (or compound) ones,

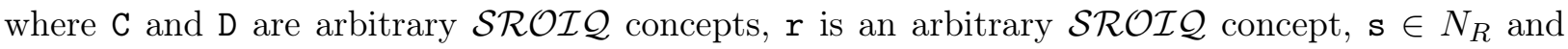
$a \in N_{I}$ [BHLS17].

A $\mathcal{S R O \mathcal { I } Q}$ role is either a term in $\mathrm{r} \in N_{R}$ or its inverse $\mathrm{r}^{-}$.

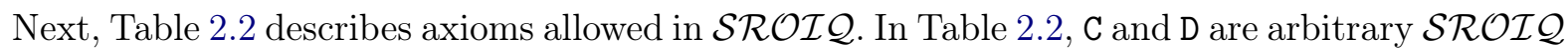

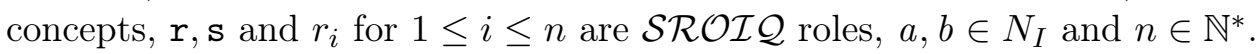

In addition to the elements presented, $\mathcal{S} \mathcal{R O} \mathcal{I} \mathcal{Q}(\mathcal{D})$ (which underpins OWL 2 DL) also includes datatypes, whose interpretation is domain specific. For more details on datatypes and their interpretation, the reader is referred to [BHLS17]. Further, concepts in DLs correspond to OWL 2 classes, roles to object properties and individuals to individuals. Throughout this thesis, the DL and OWL 2 nomenclature will be used interchangeably.

Finally, we consider that an ontology is a set of axioms on a given Description Logic. The $\mathcal{O}$ corresponds to the simplest DL (using as few extensions as possible) on which all the axioms of $\mathcal{O}$ can be expressed. 


\begin{tabular}{|c|c|c|}
\hline Name & Syntax & Semantic \\
\hline Conjunction & $\mathrm{C} \sqcap \mathrm{D}$ & $\mathrm{C}^{\mathcal{I}} \cap \mathrm{D}^{\mathcal{I}}$ \\
\hline Disjunction & $\mathrm{C} \sqcup \mathrm{D}$ & $\mathrm{C}^{\mathcal{I}} \cup \mathrm{C}^{\mathcal{I}}$ \\
\hline Negation & $\neg \mathrm{C}$ & $\Delta^{\mathcal{I}} \backslash C^{\mathcal{I}}$ \\
\hline Existential Restriction & $\exists r . C$ & $\left\{a \in \Delta^{\mathcal{I}} \mid \exists b .(a, b) \in \mathrm{r}^{\mathcal{I}} \wedge b \in \mathrm{C}^{\mathcal{I}}\right\}$ \\
\hline Value Restriction & $\forall \mathrm{r} . \mathrm{C}$ & $\left\{a \in \Delta^{\mathcal{I}} \mid \forall b .(a, b) \in \mathrm{r} \rightarrow b \in \mathrm{C}^{\mathcal{I}}\right\}$ \\
\hline Self Restriction & $\exists \mathrm{s} . \mathrm{Self}$ & $\left\{\right.$ ind $\left.a \in \Delta^{\mathcal{I}} \operatorname{mid}(a, a) \in s^{\mathcal{I}}\right\}$ \\
\hline \multirow[t]{2}{*}{ Qualified Number Restriction } & $\geq n \mathrm{~s} . \mathrm{C}$ & $\left\{a \in \mathrm{C}^{\mathcal{I}} \mid \#\left\{b \in \Delta^{\mathcal{I}} \mid(a, b) \in \mathrm{r}^{\mathcal{I}}\right\} \geq n\right\}$ \\
\hline & $\leq n \mathrm{~s} . \mathrm{C}$ & $\left\{a \in \mathrm{C}^{\mathcal{I}} \mid \#\left\{b \in \Delta^{\mathcal{I}} \mid(a, b) \in \mathrm{r}^{\mathcal{I}}\right\} \leq n\right\}$ \\
\hline Nominals & $\{a\}$ & $\{a\}^{\mathcal{I}} \subseteq \Delta^{\mathcal{I}}$ with $\#\{a\}^{\mathcal{I}}=1$ \\
\hline
\end{tabular}

Table 2.1: Complex concepts in $\mathcal{S R O I \mathcal { Q }}$

\begin{tabular}{lll}
\hline Name & Syntax & Semantics \\
\hline Concept inclusion & $\mathrm{C} \sqsubseteq \mathrm{D}$ & $\mathrm{C}^{\mathcal{I}} \subseteq \mathrm{D}^{\mathcal{I}}$ \\
Concept equality & $\mathrm{C} \equiv \mathrm{D}$ & $\mathrm{C}^{\mathcal{I}}=\mathrm{D}^{\mathcal{I}}$ \\
Concept assertion & $\mathrm{C}(a)$ & $a^{\mathcal{I}} \in \mathrm{C}^{\mathcal{I}}$ \\
Role assertion & $\mathrm{r}(a, b)$ & $\left(a^{\mathcal{I}}, b^{\mathcal{I}}\right) \in \mathrm{r}^{\mathcal{I}}$ \\
Complex role inclusion & $\mathrm{r}_{1} \circ \cdots \circ \mathrm{r}_{\mathrm{n}} \sqsubseteq \mathrm{s}$ & $\mathrm{r}_{1}^{\mathcal{I}} \circ \cdots \circ \mathrm{r}_{n}^{\mathcal{I}} \sqsubseteq \mathrm{s}^{\mathcal{I}}$ \\
Disjoint roles & $\operatorname{Disj}(\mathrm{r}, \mathrm{s})$ & $\mathrm{r}^{\mathcal{I}} \cap \mathbf{s}^{\mathcal{I}}=\emptyset$ \\
Transitivity & $\operatorname{Trans}(\mathrm{r})$ & $\left(\mathrm{r}^{+}\right)^{\mathcal{I}}=\mathrm{r}^{\mathcal{I}}$ \\
Reflexivity & $\operatorname{Refl}(\mathrm{r})$ & $\forall a \in \Delta^{\mathcal{I}},(a, a) \in \mathrm{r}^{\mathcal{I}}$ \\
Irreflexivity & $\operatorname{Irref}(\mathrm{r})$ & $\forall a \in \Delta^{\mathcal{I}},(a, a) \notin \mathrm{r}^{\mathcal{I}}$ \\
Symmetry & $\operatorname{Sym}(\mathrm{r})$ & $\left(a^{\mathcal{I}}, b^{\mathcal{I}}\right) \in \mathrm{r}^{\mathcal{I}} \Longrightarrow\left(b^{\mathcal{I}}, a^{\mathcal{I}}\right) \in \mathrm{r}^{\mathcal{I}}$ \\
Antisymmetry & $\operatorname{ASym}(\mathrm{r})$ & $\left(a^{\mathcal{I}}, b^{\mathcal{I}}\right) \in \mathrm{r}^{\mathcal{I}} \Longrightarrow\left(b^{\mathcal{I}}, a^{\mathcal{I}}\right) \notin \mathrm{r}^{\mathcal{I}}$ \\
\hline
\end{tabular}

Table 2.2: Axioms in $\mathcal{S R O I Q}$

Finally, in this thesis, an ontology $\mathcal{O}$ is a set of axioms in a description logic not more expressive than $\mathcal{S R O I Q ( D )}$. The language of the ontology $\mathcal{O}$ corresponds to the smallest description logic that can express all axioms in $\mathcal{O}$.

Example 2.2.1 illustrates how to represent ontology from Figure 1.1 in $\mathcal{S R O I \mathcal { ~ }}$

Example 2.2.1. The ontology MovieEx below represents the same concepts as Figure 1.1.

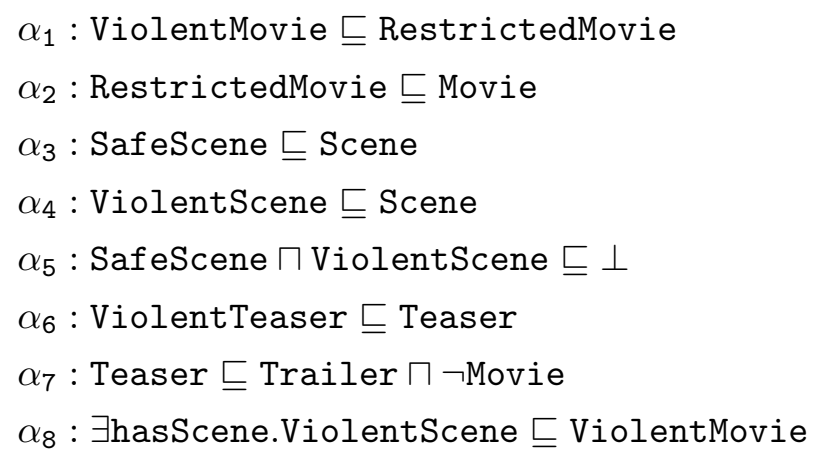




\subsection{Belief Base Change in Description Logics}

The subject matter of Belief Revision is the acquisition of new information by rational agents and how these entities update their beliefs in consequence. In presence of new information, intelligent entities often have to review their current belief state (their view of the world), possibly discarding previous beliefs given new evidence. It is not a trivial task to determine the principles and exact characteristics of such process (especially about which of the previous beliefs should be kept or to given up). Some questions arise about what can be deemed rational, how to represent a belief state of an agent and how to effectively devise rational procedures of change.

Thus, many researchers tried to formalize the process of belief revision taking into account different standpoints about rationality, using distinct approaches to represent belief states (notably belief bases) and each attacking a different facet about it (iteration, multiple revision, underlying logics) [Han99, FH11]. However, the seminal work in this area is due to Alchourrón, Gärdenfors and Makinson [AGM85] and it is discussed in the next subsection.

\subsubsection{The AGM Paradigm}

The formalization proposed by Alchourrón, Gärdenfors and Makinson [AGM85], the AGM paradigm, is used as a foundation for many theories in Belief Change. In their framework, the beliefs are represented by belief sets: sets of sentences in some logic $\mathfrak{L}$ (each sentence corresponds to a single belief) closed under a consequence operation $(\mathrm{Cn})$.

The AGM paradigm requires a consequence operation that is Tarskian (satisfies monotonicity, idempotence and inclusion), compact, satisfies the deduction theorem and is supraclassical (such requirements over the consequence operation are also known as AGM assumptions) [RW09].

The model defines three operations of change over belief sets: expansion, contraction and revision. The simplest one is expansion $(K+\varphi)$, where a new formula is added directly to the belief set, and then the set is closed under Cn. Contraction $(K-\varphi)$ removes from $K$ some formula $\varphi$, in such way that the remaining belief set does not imply $\varphi$. Finally, in revision $(K * \varphi)$, the resulting set must imply a given sentence $\varphi$ that may be inconsistent with $K$, so anything that conflicts with $\varphi$ must be removed from $K$ beforehand.

Of the three basic operations, only expansion is uniquely determined. Contraction and revision instead are constrained via sets of postulates [AGM85]. In both operations, some formulas might be removed, and thus, rarely a single plausible option appears. Even so, this opens the way to arguably one of the most defining aspects of the AGM theory: the rationality postulates.

Although there can be multiple ways to construct contraction and revision functions, not all of them can be considered rational. The AGM theory constrains the behaviour of these functions via sets of rationality postulates that represent the restrictions on the reasoning that an intelligent should adhere when adapting his knowledge to a particular input. These postulates relate to actual mathematical constructions that generate such functions via representation theorems (these show that a function satisfies the postulates iff it is built using a given construction) [AGM85]. Not only that, there is a close relation too with constructions defined via models (e.g. systems of spheres) and orderings over formulas and belief sets [Han99, FH11].

The original paradigm has evolved in different directions, being adapted to different forms of change (such as multiple and iterated), representations of epistemic states (e.g. belief bases), and logics [Pep08, FH11].

Among the ramifications of the AGM theory, two are crucial in this study: the Belief Bases as proposed by Hansson [Han94b] and its adaptation for Description Logics as presented by Ribeiro [Rib13]. Both variants are the focus of Subsection 2.3.2.

\subsubsection{From AGM to Description Logic Belief Bases}

Hansson [Han91, Han94b] advocates the use of belief bases instead of belief sets to, among other benefits, differentiate explicit and implicit beliefs and have a more feasible representation of 
epistemic states. Belief bases are a generalisation of belief sets in that they drop the need to be closed under $\mathrm{Cn}$. As with belief sets, given a belief base $B, \varphi$ is believed in $B$ if $\varphi \in \operatorname{Cn}(B)$. Regarding belief bases, this thesis follows the formalization proposed in [Han93], where an operation of contraction and two kinds of revision (internal and external) are defined for these sets. Both operations are also characterised via a set of postulates and mapped to corresponding mathematical formulations via representation theorems.

Continuing the developments in Belief Bases, Hansson and Wassermann [HW02] pruned the number of properties that a logic must have to be compatible with the belief base operations initially proposed by Hansson [Han94b]. In particular, for contraction the logics needs only to satisfy monotonicity and compactness, for revision (both internal and external), the logic must also satisfy $\alpha$-local non-contravention. A logic $\mathfrak{L}$ satisfies $\alpha$-local non-contravention iff for every $\alpha \in \mathfrak{L}$ and every $A \subseteq \mathfrak{L}$, it holds that $\alpha \notin \operatorname{Cn}(A)$, then $\alpha \notin \operatorname{Cn}(A \cup\{\neg \alpha\})$ [Was00].

The Description Logics do not satisfy $\alpha$-local non-contravention in general. This is mostly due to the lack of a classical negation of formulas. For instance, the meaning of a negation of a subsumption (such as $\mathrm{A} \sqsubseteq \mathrm{B}$ ) is still unclear. To circumvent these restrictions, new operations have been proposed, both belief sets and belief bases [Flo06, RW09, Rib13]. Hence, studies such as this cases can operate with ontologies as finite belief bases of DL formulas (general belief bases need not to be finite, nor restricted to DLs). In Subsection 2.3.3 we introduce these operations, related constructions and a good portion of the notation used throughout this thesis.

\subsubsection{Bridging Belief Change and Ontology Repair}

The operations considered in this study are the expansion, which is simply the addition of formulas via set-union, the contraction as formalised by Hansson and Wassernamnn [HW02] and the internal and external revision operations as proposed by Ribeiro [Rib13]. Before going to the mathematical definitions and postulates, this subsection discusses the notation adopted, which differs from the traditional notation found Belief Change's studies.

In Belief Change, there are two main methods of generating contraction and revision functions satisfying the rationality postulates: the partial meet and the kernel approach. In the partial meet approach, a contraction or revision depends on a selection function, which selects a subset of the maximal subsets of a belief base (or set) that do not imply a particular formula (called remainder set) [AGM85]. Dually, in the kernel approach the functions rely instead on an incision function that removes at least one formula from each element in the set of minimal subsets of the belief base (or set) that imply a certain formula (called kernel set) [Han94a].

In Ontology Repair, both kernel sets and remainder sets also play an important role, although they assume other names [MGSW19]. Figure 2.1 exhibits the relationship between terms in Belief Change and Ontology Repair with the sets of minimal implicants (MinImp) and maximal nonimplicants (MaxNon) as defined in [MGSW19].

This thesis extends the notation presented by Matos et al. [MGSW19] instead of adopting a more traditional approach because of problems with the usual notation in Belief Change and lack of a commonly agreed naming scheme in Ontology Repair.

The symbols for contraction and revision are not standardised; there is a considerable variation in the literature. Also, while compact, some of these symbols (e.g. $\downarrow, \Perp$ ) do not aid in recalling the operation or construction they refer to and are hard to communicate. Moreover, contraction and revision symbols are often accompanied by a subscript of ambiguous interpretation. This subscript can be used either to tell if the contraction is based on partial meet (subscript $\gamma$ ) or kernel (subscript $\sigma$ ); or to indicate the actual function that constitutes in its definition (e.g. $\sigma_{1}, \gamma_{f u l l}$ ).

Another aggravating factor is that the notation sometimes omits parameters that are part of the function definition. While this might help to keep the notation clean, sometimes it can make the meaning of operations unclear, especially to non-experts in the area. For instance, Ribeiro [Rib13] defines an arbitrary but fixed set of unwanted sentences $\Omega$ that figures in the definition of the versions of MinImp and MaxNon. Such parameter, however, is not made explicit in the notation of this construction, here such parameter is made explicit, and this fact aid us in showing that revision 


\section{Ontology Repair}

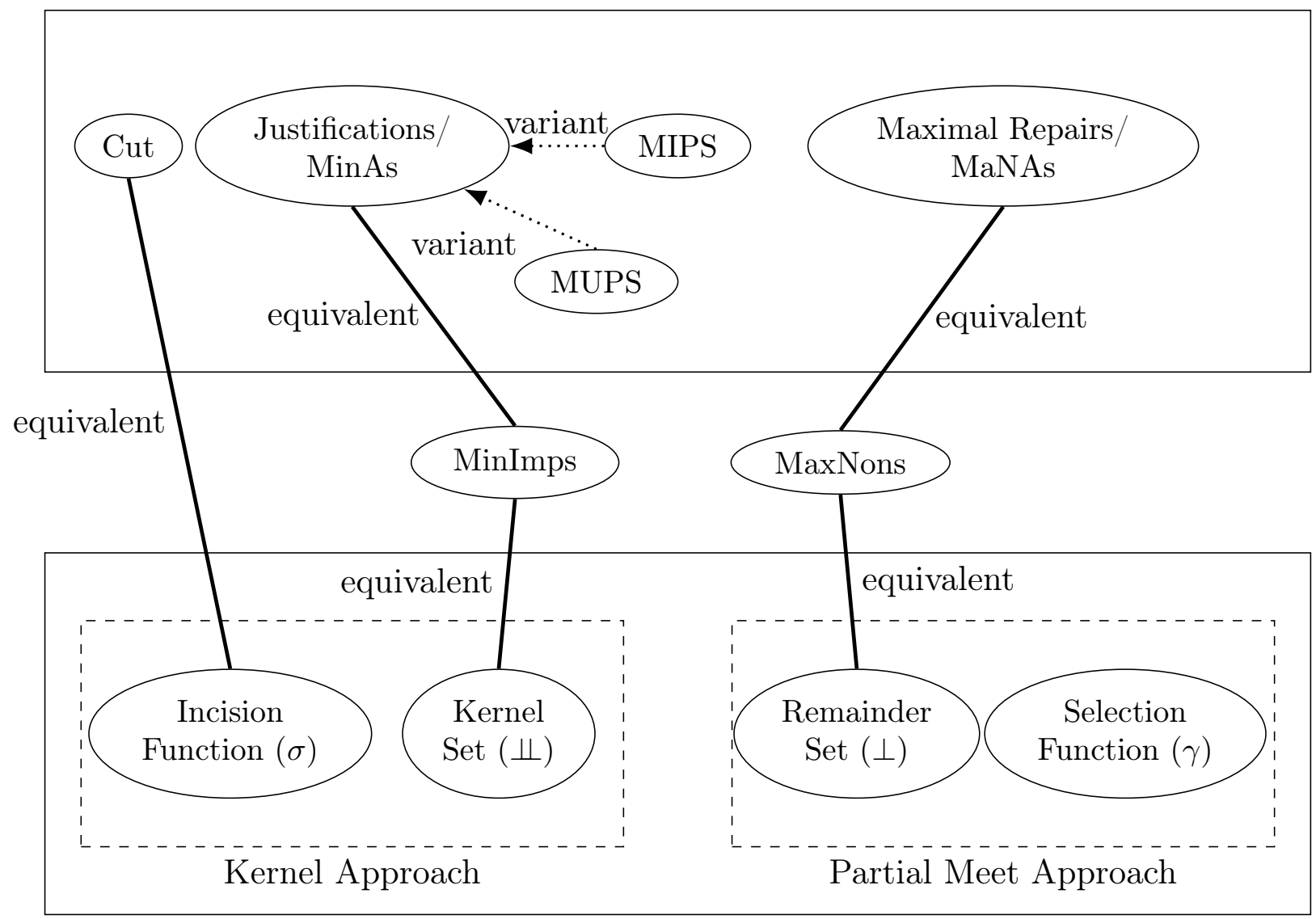

Belief Change

Figure 2.1: Relationship between concepts in Belief Change and Ontology Repair [MGSW19]

is a generalisation of contraction in this setting. As a bonus, moving from the infix notation to the prefixed makes the notation closer to the one employed in Ontology Repair [SC03, Hor11].

As for the terminology and notation for Ontology Repair two issues stand out: the multiplicity of names and the usage of acronyms (with a few exceptions). While simply choosing one of the many names could solve the problem locally, the acronyms ask the reader, potentially new to the area, to remember whether "M" means minimal or maximal, if "S" means set or subsets. Moreover, while the name "justifications" is adequate when the purpose is to explain an entailment, it loses its precise meaning when the objective is a repair.

The first component that will appear in many of future definitions is the setting (Definition 2.3.1), which is merely a succinct way to encapsulate the arguments of a Belief Change operation or goal in an Ontology Repair while at the same time making the restrictions on the arguments explicit.

Definition 2.3.1 (Setting). A setting is a tuple $(\mathfrak{L}, \mathcal{O}, \Omega, \Phi)$ where $\mathfrak{L}$ is a logic, $\mathcal{O} \in \mathcal{P}(\mathfrak{L})$ and $\Omega, \Phi \in \mathcal{P}_{\text {fin }}(\mathfrak{L})$. A setting is a DL-setting if $\mathfrak{L}$ is a DL. Also, the setting is finite if $\mathcal{O} \in \mathcal{P}_{\text {fin }}(\mathfrak{L})$.

In Definition 2.3.1, $\mathfrak{L}$ defines the scope of the other parameters, $\mathcal{O}$ is the starting point, $\Omega$ is a set of forbidden entailments and $\Phi$ is the set of formulas taken as input. Ribeiro [Rib13] proposed the usage of $\Omega$ to represent the unwanted formulas as a way to define Belief Change constructions in logics without classical negation. Further, the main purpose of such set was to represent inconsistency or incoherence as faulty consequences. However, instead of parametrising the operations in $\Omega$, the author assumes that $\Omega$ is fixed and omits it from the notation. In this thesis, not only $\Omega$ will be an arbitrary, but finite set of sentences, but will also be a parameter for contraction and revision operations, adding another degree of flexibility. 
The fourth argument of a setting, $\Phi$, may also contain the formulas in $\mathcal{O}$ which must be preserved. This idea is explored by Matos et al. [MGSW19] to capture the Gentle Repairs from Baader et al. [BKNP18]. The same strategy can be applied to other works which assume a set of protected formulas such as the multiple revision approach via core beliefs due to Yuan, Ju and Feng [YJW14].

Throughout this thesis, the language $\mathfrak{L}$ is assumed to satisfy compactness and monotonicity, unless stated otherwise. The consequence operation $\mathrm{Cn}_{\mathfrak{L}}$ also must satisfy idempotence and inclusion.

As mentioned, the notation employed in this study is a variation from the one proposed by Matos et al. [MGSW19]. The first change consists in putting MaxNon and MinImp in their plural form, making it easier to remember that MaxNons and MinImps are sets of sets. The original "singular" form will be used to refer to individual elements (e.g. a single minimal subset of the ontology that implies an entailment). The second is the generalisation of constructions that accept only a single formula as input to allow a set of formulas instead. The set of input formulas is handled as a single formula, in the same way as done by Ribeiro [Rib13]. Definitions 2.3.2 and 2.3.4 present the resulting definitions.

Definition 2.3.2 (Minimal Implying Subsets [MGSW19]). Let $(\mathfrak{L}, \mathcal{O}, \Omega, \Phi$ ) be a setting (as in Definition 2.3.1). The set of minimal $\Omega$-implying subsets of $\mathcal{O}$ with respect to $\Phi$, denoted by $\operatorname{MinImps}(\mathcal{O}, \Omega, \Phi)$, is the set such that $X \in \operatorname{MinImps}(\mathcal{O}, \Omega, \Phi)$ iff:

1. $X \subseteq \mathcal{O}$.

2. $\operatorname{Cn}(X \cup \Phi) \cap \Omega \neq \emptyset$.

3. If $X^{\prime} \subset X$, then $\operatorname{Cn}(X \cup \Phi) \cap \Omega=\emptyset$.

Example 2.3.3 contains a small example of MinImps.

Example 2.3.3. Consider the ontology $\mathcal{O}_{e x 1}$ below.

$$
\begin{aligned}
\mathcal{O}_{\text {ex1 }}=\{ & \\
& \alpha_{1}: \mathrm{A} \sqsubseteq \mathrm{B}, \quad \alpha_{2}: \mathrm{A} \sqsubseteq \mathrm{C}, \\
& \alpha_{3}: \mathrm{D} \sqsubseteq \mathrm{E}, \quad \alpha_{4}: \mathrm{D} \equiv \exists \mathrm{r} . \mathrm{Y}, \\
& \left.\alpha_{5}: \mathrm{X} \sqsubseteq \mathrm{Y}, \quad \alpha_{6}: \mathrm{r} \sqsubseteq \mathrm{s}\right\}
\end{aligned}
$$

Now, suppose that a user wants to add the set of formulas $\Phi=\{\mathrm{B} \sqsubseteq \exists \mathrm{s} . \mathrm{X}, \mathrm{C} \sqsubseteq \mathrm{D}\}$ to this ontology but, at the same time, does not want the entailments in $\Omega=\{\mathrm{A} \sqsubseteq \mathrm{D}, \mathrm{D} \sqsubseteq \perp\}$. Then, the subsets of the ontology which will cause entailments in $\Omega$ with the addition of $\Phi$ are $\operatorname{MinImps}\left(\mathcal{O}_{e x 1}, \Omega, \Phi\right)=$ $\left\{\left\{\alpha_{2}\right\},\left\{\alpha_{1}, \alpha_{4}, \alpha_{5}, \alpha_{6}\right\}\right\}$.

When $\Phi$ is a singleton, this definition matches the traditional "MinImp" [MGSW19], and thus the equivalences to other terms in Belief Change and Ontology Repair can be seen in Figure 2.1. In particular, the $\alpha$-kernel set of $\mathcal{O}$ (in the traditional sense) corresponds to $\operatorname{Min} \operatorname{Imps}(\mathcal{O},\{\alpha\}, \emptyset)$. This set also corresponds to the set of all justifications of $\mathcal{O}$ for $\alpha$ [MGSW19]. Definition 2.3.4 introduces its dual construction.

Definition 2.3.4. Let $(\mathfrak{L}, \mathcal{O}, \Omega, \Phi)$ be a setting (as in Definition 2.3.1). The set of maximal $\Omega$ non-implying subsets of $\mathcal{O}$ with respect to $\Phi$, denoted by $\operatorname{MaxNons}(\mathcal{O}, \Omega, \Phi)$, is the set such that $X \in \operatorname{MaxNons}(\mathcal{O}, \Omega, \Phi)$ iff:

1. $X \subseteq \mathcal{O}$.

2. $\operatorname{Cn}(X \cup \Phi) \cap \Omega=\emptyset$.

3. If $X \subset X^{\prime} \subseteq \mathcal{O}$, then $\operatorname{Cn}(X \cup \Phi) \cap \Omega \neq \emptyset$. 
Example 2.3.5. Consider the ontology $\mathcal{O}_{\text {ex } 1}$ from Example 2.3.3. Using the same sets $\Phi$ and $\Omega$, the MaxNons obtained are:

$$
\operatorname{MaxNons}\left(\mathcal{O}_{e x 1}, \Omega, \Phi\right)=\left\{\mathcal{O}_{e x 1} \backslash\left\{\alpha_{2}, \alpha_{1}\right\}, \mathcal{O}_{e x 1} \backslash\left\{\alpha_{2}, \alpha_{4}\right\}, \mathcal{O}_{e x 1} \backslash\left\{\alpha_{2}, \alpha_{5}\right\}, \mathcal{O}_{e x 1} \backslash\left\{\alpha_{2}, \alpha_{6}\right\}\right\}
$$

Again, when $\Phi$ is a singleton, this definition matches the traditional "MaxNon" [MGSW19], and thus the equivalences to other terms are shown in Figure 2.1. In particular, the $\alpha$-remainder set of $\mathcal{O}$ (also in the traditional sense) corresponds to $\operatorname{MaxNons}(\mathcal{O},\{\alpha\}, \emptyset)$. This set also corresponds to the set of optimal classical repair of $\mathcal{O}$ for $\alpha$ [MGSW19].

Next comes the presentation of the contraction and revision functions. Before each, however, another component must be defined, which takes the form of a incision function for MinImps-based (kernel) approaches or a selection function for MaxNons-based (partial meet approaches). The postulates are presented even though they already appear many times in the literature not only for self-containment but also to avoid misunderstandings when considering the notation employed.

Definition 2.3.6 (Incision function (without protection) [Han94a, Rib13]). Let $(\mathfrak{L}, \mathcal{O}, \Omega, \Phi)$ be a setting (as in Definition 2.3.1), a function $\mathbf{f}: \mathcal{P}(\mathcal{P}(\mathfrak{L})) \times \mathcal{P}_{\text {fin }}(\mathfrak{L}) \times \mathcal{P}_{\text {fin }}(\mathfrak{L}) \rightarrow \mathcal{P}(\mathcal{O})$ is an incision function (without protection) for the setting $(\mathfrak{L}, \mathcal{O}, \Omega, \Phi)$ iff:

1. $\mathbf{f}(\operatorname{MinImps}(\mathcal{O}, \Omega, \Phi), \Omega, \Phi) \subseteq \bigcup \operatorname{MinImps}(\mathcal{O}, \Omega, \Phi)$.

2. $\mathbf{f}(\operatorname{MinImps}(\mathcal{O}, \Omega, \Phi), \Omega, \Phi) \cap X \neq \emptyset$ for all $X \in \operatorname{MinImps}(\mathcal{O}, \Omega, \Phi)$ such that $X \neq \emptyset$.

Definition 2.3.6 corresponds to the classical formulation of an incision function, and the primary requisite is that it must remove at least one element from each MinImp. Incision function as such form the kernel contraction of Definition 2.3.7.

Definition 2.3.7 (Kernel contraction [Han94a, HW02]). Let $\mathbf{f}$ be a incision function (without protection) as in Definition 2.3.6 for a setting $(\mathfrak{L}, \mathcal{O}, \Omega, \Phi)$, then:

$$
\operatorname{kc}_{\mathbf{f}}(\mathcal{O}, \Omega)=\mathcal{O} \backslash \mathbf{f}(\operatorname{MinImps}(\mathcal{O}, \Omega, \emptyset), \Omega, \emptyset)
$$

is a kernel contraction for $(\mathfrak{L}, \mathcal{O}, \Omega, \Phi)$.

Kernel contraction is characterised by a set of postulates via the following theorem.

Theorem 2.3.8 (Representation theorem for kernel base contraction [HW02]). Let $\mathfrak{L}$ be a monotonic and compact logic and $(\mathfrak{L}, \mathcal{O}, \Omega, \Phi)$ a setting. Then con is a kernel contraction for the setting $(\mathfrak{L}, \mathcal{O}, \Omega, \Phi)$ iff it satisfies the following postulates:

inclusion: $\operatorname{con}(\mathcal{O}, \Omega) \subseteq \mathcal{O}$.

success: $\operatorname{Cn}(\operatorname{con}(\mathcal{O}, \Omega)) \cap \Omega=\emptyset$.

uniformity: If for every subset $\mathcal{O}^{\prime}$ of $\mathcal{O}$ it holds that $\operatorname{Cn}\left(\mathcal{O}^{\prime}\right) \cap \Omega \neq \emptyset$ iff $\operatorname{Cn}\left(\mathcal{O}^{\prime}\right) \cap \Omega \neq \emptyset$, then $\operatorname{con}(\mathcal{O}, \Omega)=\operatorname{con}\left(\mathcal{O}, \Omega^{\prime}\right)$

core-retainment: If $\alpha \in \mathcal{O} \backslash \operatorname{con}(\mathcal{O}, \Omega)$ then there is an $\mathcal{O}^{\prime}$ such that $\mathcal{O}^{\prime} \subseteq \mathcal{O}$ and $\operatorname{Cn}\left(\mathcal{O}^{\prime}\right) \cap \Omega=\emptyset$, but $\operatorname{Cn}\left(\mathcal{O}^{\prime} \cup\{\alpha\}\right) \cap \Omega \neq \emptyset$.

Matos et al. [MGSW19] prove the relation of Definition 2.3.6 with justification-based ontology repair. In particular, they show that optimal (maximal) repairs are simply kernel contractions with minimal incision functions (Definition 2.3.9).

Definition 2.3.9 (Minimal incision function [FFK06]). Let $\mathbf{f}$ be an incision function for the setting $(\mathfrak{L}, \mathcal{O}, \Omega, \Phi)$. Then $\mathbf{f}$ is minimal if no other incision function $\mathbf{f}^{\prime}$ for $(\mathfrak{L}, \mathcal{O}, \Omega, \Phi)$ is such that: $\mathbf{f}^{\prime}(\operatorname{MinImps}(\mathcal{O}, \Omega, \Phi), \Omega, \Phi) \subseteq \mathbf{f}(\operatorname{MinImps}(\mathcal{O}, \Omega, \Phi), \Omega, \Phi)$. 
For the dual of kernel contractions, that is partial meet contractions, instead of an incision function, the formulation depends on a selection function as in Definition 2.3.10.

Definition 2.3.10 (Selection function (without protection) [AGM85, Rib13]). Let $(\mathfrak{L}, \mathcal{O}, \Omega, \Phi)$ be a setting (as in Definition 2.3.1), a function $\mathbf{g}: \mathcal{P}(\mathcal{P}(\mathfrak{L})) \times \mathcal{P}_{\text {fin }}(\mathfrak{L}) \times \mathcal{P}_{\text {fin }}(\mathfrak{L}) \rightarrow \mathcal{P}(\mathcal{P}(\mathfrak{L})$ ) is a selection function (without protection) for the setting $(\mathfrak{L}, \mathcal{O}, \Omega, \Phi)$ iff:

1. $\emptyset \neq \mathbf{g}(\operatorname{MaxNons}(\mathcal{O}, \Omega, \Phi), \Omega, \Phi) \subseteq \operatorname{MaxNons}(\mathcal{O}, \Omega, \Phi)$, if $\operatorname{MaxNons}(\mathcal{O}, \Omega, \Phi) \neq \emptyset$.

2. Otherwise, $\mathbf{g}(\operatorname{MaxNons}(\mathcal{O}, \Omega, \Phi), \Omega, \Phi)=\{\mathcal{O}\}$.

Definition 2.3.10 corresponds to the classical formulation of selection functions, and the primary requirement is that it must return a non-empty subset of the MaxNons, if possible, and the ontology itself otherwise. Selection functions are essential components of the partial meet contractions from Definition 2.3.11.

Definition 2.3.11 (Partial meet contraction [AGM85]). Let $\mathbf{g}$ be a selection function (without protection) as in Definition 2.3.10 for a setting $(\mathfrak{L}, \mathcal{O}, \Omega, \Phi)$, then:

$$
\operatorname{pmc}_{\mathbf{f}}(\mathcal{O}, \Omega)=\bigcap \mathbf{g}(\operatorname{MaxNons}(\mathcal{O}, \Omega, \emptyset), \Omega, \emptyset)
$$

is a partial meet contraction for $(\mathfrak{L}, \mathcal{O}, \Omega, \Phi)$.

Partial meet contraction is characterised by a set of postulates via the following theorem.

Theorem 2.3.12 (Representation theorem for partial meet base contraction [HW02]). Let $\mathfrak{L}$ be a monotonic and compact logic and $(\mathfrak{L}, \mathcal{O}, \Omega, \Phi)$ a setting. Then con is a partial contraction for the setting $(\mathfrak{L}, \mathcal{O}, \Omega, \Phi)$ iff it satisfies the following postulates:

inclusion: $\operatorname{con}(\mathcal{O}, \Omega) \subseteq \mathcal{O}$.

success: $\operatorname{Cn}(\operatorname{con}(\mathcal{O}, \Omega)) \cap \Omega=\emptyset$.

uniformity: If for every subset $\mathcal{O}^{\prime}$ of $\mathcal{O}$ it holds that $\operatorname{Cn}\left(\mathcal{O}^{\prime}\right) \cap \Omega \neq \emptyset$ iff $\operatorname{Cn}\left(\mathcal{O}^{\prime}\right) \cap \Omega \neq \emptyset$, then $\operatorname{con}(\mathcal{O}, \Omega)=\operatorname{con}\left(\mathcal{O}, \Omega^{\prime}\right)$

relevance: If $\alpha \in \mathcal{O} \backslash \operatorname{con}(\mathcal{O}, \Omega)$ then there is an $\mathcal{O}^{\prime}$ such that $\operatorname{con}(\mathcal{O}, \Omega) \subseteq \mathcal{O}^{\prime} \subseteq \mathcal{O}$ and $\operatorname{Cn}\left(\mathcal{O}^{\prime}\right) \cap \Omega=$ $\emptyset$, but $\operatorname{Cn}\left(\mathcal{O}^{\prime} \cup\{\alpha\}\right) \cap \Omega \neq \emptyset$.

Since relevance implies core-retainment, it becomes clear from the postulates in Theorems 2.3.8 and 2.3.12 that kernel contractions are more general than their partial meet counterparts. However, in a similar way as Falappa, Fermé and Kern-Isberner [FFK06] prove for classical logics, Matos et al. prove that for DLs, the partial meet functions are equivalent to the kernel contractions built using regular incision functions [MGSW19]. Regular incision functions are the union of minimal incision functions, and this is sufficient to guarantee relevance. Moreover, an optimal (maximal) repair can also be attained via partial meet functions using maxichoice selection functions, that is, selection functions $\mathbf{g}$ such that $|\mathbf{g}(\operatorname{MaxNons}(\mathcal{O}, \Omega, \Phi), \Omega, \Phi)|=1$.

Up to now, the definitions ignore the third argument of MinImps and MaxNons. This is because contraction only needs one set of formulas as input. However, in revision, they play a central role. In the traditional notation for revision there was no specification of $\Omega$, in logics with negation it simply corresponded to a fixed inconsistent formula, the only argument in addition to the initial base was the set of formulas to be added. Ribeiro [Rib13] defined it as fixed set that could represent either inconsistencies or formulas that represent unsatisfiable concepts (e.g. A $\sqsubseteq \perp$ for a atomic concept A). As mentioned before, the version presented here generalises this notion, allowing it to represent inconsistencies, incoherences and any other formula that is deemed unwanted at will. In this way, revision can be understood in some cases as a generalisation of contraction.

The first type of revision discussed in the internal kernel revision as in Definition 2.3.13. 
Definition 2.3.13 (Internal kernel revision [Rib13]). Let $\mathbf{f}$ be a incision function (without protection) as in Definition 2.3.6 for a setting $(\mathfrak{L}, \mathcal{O}, \Omega, \Phi)$, then:

$$
\operatorname{ikr}_{\mathbf{f}}(\mathcal{O}, \Omega, \Phi)=\Phi \cup(\mathcal{O} \backslash \mathbf{f}(\operatorname{MinImps}(\mathcal{O}, \Omega, \Phi), \Omega, \Phi))
$$

is a internal kernel revision for $(\mathfrak{L}, \mathcal{O}, \Omega, \Phi)$.

Internal kernel revision is also characterised by a set of postulates as shown in Theorem 2.3.14.

Theorem 2.3.14 (Representation theorem for internal kernel base revision [Rib13]). Let $\mathfrak{L}$ be a monotonic and compact logic and $(\mathfrak{L}, \mathcal{O}, \Omega, \Phi)$ a setting. Then rev is a internal kernel revision for the setting $(\mathfrak{L}, \mathcal{O}, \Omega, \Phi)$ iff it satisfies the following postulates:

inclusion: $\operatorname{rev}(\mathcal{O}, \Omega, \Phi) \subseteq \operatorname{ex}(\mathcal{O}, \Phi)$.

strong success: $\Phi \subseteq \operatorname{rev}(\mathcal{O}, \Omega, \Phi)$.

weak consistency: If $\operatorname{Cn}(\Phi) \cap \Omega=\emptyset$, then $\operatorname{Cn}(\operatorname{rev}(\mathcal{O}, \Omega, \Phi)) \cap \Omega=\emptyset$.

uniformity: If for every subset $\mathcal{O}^{\prime}$ of $\mathcal{O}$ it holds that $\operatorname{Cn}\left(\mathcal{O}^{\prime} \cup \Phi\right) \cap \Omega \neq \emptyset$ iff $\operatorname{Cn}\left(\mathcal{O}^{\prime} \cap \Phi^{\prime}\right) \cap \Omega \neq \emptyset$, then $\operatorname{rev}(\mathcal{O}, \Omega, \Phi)=\operatorname{rev}\left(\mathcal{O}, \Omega, \Phi^{\prime}\right)$.

core-retainment: If $\alpha \in \mathcal{O} \backslash \operatorname{rev}(\mathcal{O}, \Omega$, $\Phi)$ then there is an $\mathcal{O}^{\prime}$ such that $\mathcal{O}^{\prime} \subseteq \mathcal{O} \cup \Phi$ and $\operatorname{Cn}\left(\mathcal{O}^{\prime}\right) \cap$ $\Omega=\emptyset$, but $\operatorname{Cn}\left(\mathcal{O}^{\prime} \cup\{\alpha\}\right) \cap \Omega \neq \emptyset$.

Definition 2.3.15 presents the analogous partial meet version (MaxNons-based).

Definition 2.3.15 (Internal partial meet revision [Rib13]). Let $\mathbf{f}$ be a selection function (without protection) as in Definition 2.3.6 for a setting $(\mathfrak{L}, \mathcal{O}, \Omega, \Phi)$, then:

$$
\operatorname{ipmr}_{\mathbf{g}}(\mathcal{O}, \Omega, \Phi)=\Phi \cup \bigcap \mathbf{g}(\operatorname{MaxNons}(\mathcal{O}, \Omega, \Phi), \Omega, \Phi)
$$

is a internal partial meet revision for $(\mathfrak{L}, \mathcal{O}, \Omega, \Phi)$.

Internal partial meet revision is also characterised by a set of postulates as shown in Theorem 2.3.16.

Theorem 2.3.16 (Representation theorem for internal partial meet base revision [Rib13]). Let $\mathfrak{L}$ be a monotonic and compact logic and $(\mathfrak{L}, \mathcal{O}, \Omega, \Phi)$ a setting. Then rev is a internal kernel revision for the setting $(\mathfrak{L}, \mathcal{O}, \Omega, \Phi)$ iff it satisfies the following postulates:

inclusion: $\operatorname{rev}(\mathcal{O}, \Omega, \Phi) \subseteq \operatorname{ex}(\mathcal{O}, \Phi)$.

strong success: $\Phi \subseteq \operatorname{rev}(\mathcal{O}, \Omega, \Phi)$.

weak consistency: If $\operatorname{Cn}(\Phi) \cap \Omega=\emptyset$, then $\operatorname{Cn}(\operatorname{rev}(\mathcal{O}, \Omega, \Phi))=\emptyset$.

uniformity: If for every subset $\mathcal{O}^{\prime}$ of $\mathcal{O}$ it holds that $\operatorname{Cn}\left(\mathcal{O}^{\prime} \cup \Phi\right) \cap \Omega \neq \emptyset$ iff $\operatorname{Cn}\left(\mathcal{O}^{\prime} \cap \Phi^{\prime}\right) \cap \Omega \neq \emptyset$, then $\operatorname{rev}(\mathcal{O}, \Omega, \Phi)=\operatorname{rev}\left(\mathcal{O}, \Omega, \Phi^{\prime}\right)$.

relevance: If $\alpha \in \mathcal{O} \backslash \operatorname{rev}(\mathcal{O}, \Omega, \Phi)$ then there is an $\mathcal{O}^{\prime}$ such that $\operatorname{rev}(\mathcal{O}, \Omega, \Phi) \subseteq \mathcal{O}^{\prime} \subseteq \mathcal{O} \cup \Phi$ and $\operatorname{Cn}\left(\mathcal{O}^{\prime}\right) \cap \Omega=\emptyset$, but $\operatorname{Cn}\left(\mathcal{O}^{\prime} \cup\{\alpha\}\right) \cap \Omega \neq \emptyset$.

As with contraction functions, the kernel variant more general than its partial meet counterpart. Both internal revisions originate from the same intuitive idea of first removing anything that conflicts with the input before its addition. In contrast, the external revision operations are closer to semirevision (revision without the success postulate) in which the input is added and only after the conflicts are solved. 
Due to the nature of the external revisions, there are two categories, orthogonal to the kernel versus partial meet axis. One type with strong success (the input is always added) and weak consistency (the result does not entail anything from $\Omega$ only if the input also does not). The other satisfies weak success (the input is only accepted if it does not entail anything from $\Omega$ ) but has strong consistency (the result never entails a formula in $\Omega$ ) [Rib13].

Following the same ordering as the previous operations, the external kernel revisions are introduced first. The variant with strong success needs a different type of selection function, which is detailed in Definition 2.3.17.

Definition 2.3.17 (Incision function that protects any input [Rib13]). Let $(\mathfrak{L}, \mathcal{O}, \Omega, \Phi)$ be a setting (as in Definition 2.3.1), a function $\mathbf{f}: \mathcal{P}(\mathcal{P}(\mathfrak{L})) \times \mathcal{P}_{\text {fin }}(\mathfrak{L}) \times \mathcal{P}_{\text {fin }}(\mathfrak{L}) \rightarrow \mathcal{P}(\mathcal{O})$ is an incision function that protects any input for the setting $(\mathfrak{L}, \mathcal{O}, \Omega, \Phi)$ iff:

1. $\mathbf{f}(\operatorname{MinImps}(\mathcal{O}, \Omega, \Phi), \Omega, \Phi) \subseteq \bigcup \operatorname{MinImps}(\mathcal{O}, \Omega, \Phi)$.

2. If $\operatorname{Cn}(\Phi) \cap \Omega=\emptyset$, then $\mathbf{f}(\operatorname{MinImps}(\mathcal{O}, \Omega, \Phi), \Omega, \Phi) \cap X \neq \emptyset$ for all $X \in \operatorname{MinImps}(\mathcal{O}, \Omega, \Phi)$ such that $X \neq \emptyset$.

3. $\Phi \cap \mathbf{f}(\operatorname{MinImps}(\mathcal{O}, \Omega, \Phi), \Omega, \Phi)=\emptyset$.

The incision functions from Definition 2.3.17 provide strong success. However, as such, they cannot guarantee strong consistency: even if the input already entails formulas from $\Omega$ its formulas will never be removed, hence the result will also entail formulas from $\Omega$.

The other variant enforces consistency at all costs, even if that means rejecting the input. Still, if the input is consistent, it will be protected. Definition 2.3.18 formalises this intuition.

Definition 2.3.18 (Incision function that protects consistent inputs [Rib13]). Let $(\mathfrak{L}, \mathcal{O}, \Omega, \Phi)$ be a setting (as in Definition 2.3.1), a function $\mathbf{f}: \mathcal{P}(\mathcal{P}(\mathfrak{L})) \times \mathcal{P}_{\text {fin }}(\mathfrak{L}) \times \mathcal{P}_{\text {fin }}(\mathfrak{L}) \rightarrow \mathcal{P}(\mathcal{O})$ is an incision function that protects consistent inputs for the setting $(\mathfrak{L}, \mathcal{O}, \Omega, \Phi)$ iff:

1. $\mathbf{f}(\operatorname{MinImps}(\mathcal{O}, \Omega, \Phi), \Omega, \Phi) \subseteq \bigcup \operatorname{MinImps}(\mathcal{O}, \Omega, \Phi)$.

2. $\mathbf{f}(\operatorname{MinImps}(\mathcal{O}, \Omega, \Phi), \Omega, \Phi) \cap X \neq \emptyset$ for all $X \in \operatorname{MinImps}(\mathcal{O}, \Omega, \Phi)$ such that $X \neq \emptyset$.

3. If $\operatorname{Cn}(\Phi) \cap \Omega=\emptyset$ then, $\Phi \cap \mathbf{f}(\operatorname{MinImps}(\mathcal{O}, \Omega, \Phi), \Omega, \Phi)=\emptyset$.

The same formulation defines both types of the external kernel revision, but one with an incision function that protects any input and the other with an incision function that protects consistent inputs.Definition 2.3.19 formalises them at the same time for the MinImp-based construction (kernel), highlighting their similarities.

Definition 2.3.19 (External kernel revision [Rib13]). Let $\mathbf{f}$ be an incision function that protects any input (as in Definition 2.3.17) or an incision function that protects consistent inputs (as in Definition 2.3.18) for a setting $(\mathfrak{L}, \mathcal{O}, \Omega, \Phi)$, then:

$$
\operatorname{ekr}_{\mathbf{f}}(\mathcal{O}, \Omega, \Phi)=(\mathcal{O} \cup \Phi) \backslash \mathbf{f}(\operatorname{MinImps}(\mathcal{O} \cup \Phi, \Omega, \emptyset), \Omega, \Phi)
$$

is an external kernel revision with strong success for $(\mathfrak{L}, \mathcal{O}, \Omega, \Phi)$ if $\mathbf{f}$ protects any input or an external kernel revision with strong consistency for $(\mathfrak{L}, \mathcal{O}, \Omega, \Phi)$ if $\mathbf{f}$ protects consistent inputs.

The representation theorems for both types of external kernel revision are combined into Theorem 2.3.20.

Theorem 2.3.20 (Representation theorem for external kernel base revision [Rib13]). Let $\mathfrak{L}$ be a monotonic and compact logic and $(\mathfrak{L}, \mathcal{O}, \Omega, \Phi)$ a setting. Then rev is an external kernel revision for the setting $(\mathfrak{L}, \mathcal{O}, \Omega, \Phi)$ iff it satisfies the following postulates:

inclusion: $\operatorname{rev}(\mathcal{O}, \Omega, \Phi) \subseteq \operatorname{ex}(\mathcal{O}, \Phi)$. 
weak success: If $\operatorname{Cn}(\Phi) \cap \Omega=\emptyset$, then $\Phi \subseteq \operatorname{rev}(\mathcal{O}, \Omega, \Phi)$.

weak consistency: If $\operatorname{Cn}(\Phi) \cap \Omega=\emptyset$, then $\operatorname{Cn}(\operatorname{rev}(\mathcal{O}, \Omega, \Phi))=\emptyset$.

pre-expansion: $\operatorname{rev}(\mathcal{O}, \Omega, \Phi)=\operatorname{rev}(\mathcal{O} \cup \Phi, \Omega, \Phi)$

core-retainment: If $\alpha \in \mathcal{O} \backslash \operatorname{rev}(\mathcal{O}, \Omega, \Phi)$ then there is a $\mathcal{O}^{\prime}$ such that $\mathcal{O}^{\prime} \subseteq \mathcal{O} \cup \Phi$ and $\operatorname{Cn}\left(\mathcal{O}^{\prime}\right) \cap \Omega=$ $\emptyset$, but $\operatorname{Cn}\left(\mathcal{O}^{\prime} \cup\{\alpha\}\right) \cap \Omega \neq \emptyset$.

and at least one among:

strong success: $\Phi \subseteq \operatorname{rev}(\mathcal{O}, \Omega, \Phi)$.

strong consistency: $\operatorname{Cn}(\operatorname{rev}(\mathcal{O}, \Omega, \Phi)) \cap \Omega=\emptyset$.

Finally, the last group of operations considered in this work, the external partial meet revisions are presented in what follows. They also have the same subtypes as the external kernel revision: the functions satisfying strong success and the ones with strong consistency. Hence, they need adapted selection functions, as it was the case with the incision ones. Definition 2.3.21 describes the selection functions that yield strong success.

Definition 2.3.21 (Selection function that protects any input [Rib13]). Let $(\mathfrak{L}, \mathcal{O}, \Omega, \Phi)$ be a setting (as in Definition 2.3.1), a function $\mathbf{g}: \mathcal{P}(\mathcal{P}(\mathfrak{L})) \times \mathcal{P}_{\text {fin }}(\mathfrak{L}) \times \mathcal{P}_{\text {fin }}(\mathfrak{L}) \rightarrow \mathcal{P}(\mathcal{P}(\mathfrak{L})$ ) is a selection function that protects any input for the setting $(\mathfrak{L}, \mathcal{O}, \Omega, \Phi)$ iff:

1. If $\operatorname{Cn}(\Phi) \cap \Omega=\emptyset$, then $\emptyset \neq \mathbf{g}(\operatorname{MaxNons}(\mathcal{O}, \Omega, \Phi), \Omega, \Phi) \subseteq \operatorname{MaxNons}(\mathcal{O}, \Omega, \Phi)$ and $\Phi \subseteq$ $\bigcap \mathbf{g}(\operatorname{MaxNons}(\mathcal{O}, \Omega, \Phi), \Omega, \Phi)$.

2. Otherwise, $\mathbf{g}(\operatorname{MaxNons}(\mathcal{O}, \Omega, \Phi), \Omega, \Phi)=\{\mathcal{O}\}$.

Next, Definition 2.3.22 gives the counterpart of the previous definition, that is, modified selection functions that only protect inputs if they do not entail an undesired formula.

Definition 2.3.22 (Selection function that protects consistent inputs [Rib13]). Let $(\mathfrak{L}, \mathcal{O}, \Omega, \Phi)$ be a setting (as in Definition 2.3.1), a function $\mathbf{g}: \mathcal{P}(\mathcal{P}(\mathfrak{L})) \times \mathcal{P}_{\text {fin }}(\mathfrak{L}) \times \mathcal{P}_{\text {fin }}(\mathfrak{L}) \rightarrow \mathcal{P}(\mathcal{P}(\mathfrak{L})$ ) is a selection function that protects consistent inputs for the setting $(\mathfrak{L}, \mathcal{O}, \Omega, \Phi)$ iff:

1. $\emptyset \neq \mathbf{g}(\operatorname{MaxNons}(\mathcal{O}, \Omega, \Phi), \Omega, \Phi) \subseteq \operatorname{MaxNons}(\mathcal{O}, \Omega, \Phi)$ and $\mathbf{g}(\operatorname{MaxNons}(\mathcal{O}, \Omega, \Phi), \Omega, \Phi)=$ $\{\mathcal{O}\}$.

2. If $\operatorname{Cn}(\Phi) \cap \Omega=\emptyset$, then $\Phi \subseteq \bigcap \mathbf{g}(\operatorname{MaxNons}(\mathcal{O}, \Omega, \Phi), \Omega, \Phi)$.

External partial meet revision functions can be constructed with the same formulation, but one with a selection that protects any input and the other with an incision function that protects consistent inputs. Definition 2.3.23 formalises both.

Definition 2.3.23 (External partial meet revision [Rib13]). Let $\mathbf{g}$ be a selection function that protects any input (as in Definition 2.3.21) or a selection function that protects consistent inputs (as in Definition 2.3.22) for a setting $(\mathfrak{L}, \mathcal{O}, \Omega, \Phi)$, then:

$$
\operatorname{epmr}_{\mathbf{g}}(\mathcal{O}, \Omega, \Phi)=\bigcap \mathbf{g}(\operatorname{MaxNons}(\mathcal{O} \cup \Phi, \Omega, \emptyset), \Omega, \Phi)
$$

is an external partial meet revision with strong success for $(\mathfrak{L}, \mathcal{O}, \Omega, \Phi)$ if $\mathbf{g}$ protects any input or an external partial meet revision with strong consistency for $(\mathfrak{L}, \mathcal{O}, \Omega, \Phi)$ if $\mathbf{g}$ protects consistent inputs.

Theorem 2.3.24 combines the representation theorems for external partial meet revision with strong success and for those with strong consistency. 
Theorem 2.3.24 (Representation theorem for external partial meet base revision [Rib13]). Let $\mathfrak{L}$ be a monotonic and compact logic and $(\mathfrak{L}, \mathcal{O}, \Omega, \Phi)$ a setting. Then rev is an external partial revision for the setting $(\mathfrak{L}, \mathcal{O}, \Omega, \Phi)$ iff it satisfies the following postulates:

inclusion: $\operatorname{rev}(\mathcal{O}, \Omega, \Phi) \subseteq \operatorname{ex}(\mathcal{O}, \Phi)$.

weak success: If $\operatorname{Cn}(\Phi) \cap \Omega=\emptyset$, then $\Phi \subseteq \operatorname{rev}(\mathcal{O}, \Omega, \Phi)$.

weak consistency: If $\operatorname{Cn}(\Phi) \cap \Omega=\emptyset$, then $\operatorname{Cn}(\operatorname{rev}(\mathcal{O}, \Omega, \Phi))=\emptyset$.

pre-expansion: $\operatorname{rev}(\mathcal{O}, \Omega, \Phi)=\operatorname{rev}(\mathcal{O} \cup \Phi, \Omega, \Phi)$

relevance: If $\alpha \in \mathcal{O} \backslash \operatorname{rev}(\mathcal{O}, \Omega, \Phi)$ then there is an $\mathcal{O}^{\prime}$ such that $\operatorname{rev}(\mathcal{O}, \Omega, \Phi) \subseteq \mathcal{O}^{\prime} \subseteq \mathcal{O} \cup \Phi$ and $\operatorname{Cn}\left(\mathcal{O}^{\prime}\right) \cap \Omega=\emptyset$, but $\operatorname{Cn}\left(\mathcal{O}^{\prime} \cup\{\alpha\}\right) \cap \Omega \neq \emptyset$.

and at least one among:

strong success: $\Phi \subseteq \operatorname{rev}(\mathcal{O}, \Omega, \Phi)$.

strong consistency: $\operatorname{Cn}(\operatorname{rev}(\mathcal{O}, \Omega, \Phi)) \cap \Omega=\emptyset$.

Note that for contraction and revisions, kernel operations are more general than partial meet ones. Also, while it is not possible to obtain an external revision that satisfies both strong success and strong consistency, it is possible to obtain a function that is at the same time definable as external revision with strong success and as internal revision, thus satisfying both uniformity and pre-expansion.

Also, in the revisions construed as Definitions 2.3 .13 and 2.3 .15 , by setting $\Phi=\emptyset$ the results correspond to the constructions from Definitions 2.3.7 and 2.3.11, respectively. The same occurs with Definitions 2.3.19 and 2.3.23.

The next subsection discusses the methods to compute these operations, in particular, MinImps and MaxNons. Special attention is given to the algorithms that will compose the empirical evaluation in this thesis.

\subsubsection{Black-box Algorithms for Base Change of DL Bases}

This thesis focuses on black-box techniques to compute MinImps and MaxNons [SQJH08, JQH09, Hor11, CW15]. These approaches assume a function which returns a MinImp or MaxNon (according to which set the algorithms computes). Further, they use reasoners as an "oracle" or "black-box" whose only function is to decide whether a set of axioms entails a formula.

There are works which depend on the reasoning method. Usually, these strategies modify the reasoning mechanism to keep track of axioms used in the proof for an entailment. Examples of such proposals are the algorithms to compute MinImps by Schlobach and Cornet [SC03], Kalyanpur et al. [KPSH05], Peñaloza [Peñ09] and Ozaki and Penãloza [OP18]. These methods are generally known as glass-box techniques.

The glass-box algorithms can have better performance since they are specifically designed to the task of building MinImps and MaxNons. However, they are much less flexible: improving one requires to change the reasoning algorithm itself. Black-box algorithms are more flexible than glassbox ones since practically any reasoner is sufficient.

Further, as current reasoners are very optimised, Horridge [Hor11] questions whether the benefits of glass-box approaches justify the additional complexity. In the evaluation of the number of entailment checks performed when computing a single MinImp, glass-box methods surpassed black-box ones by orders of magnitude only in between 1 and $10 \%$ the entailments in 3 out of 72 ontologies tested.

Hence, flexibility and simplicity are two of the factor that led to the choice of black-box approaches. Further, as it is shown later in this subsection, the algorithms for MinImps and MaxNons 
are very similar, thus allowing us to obtain useful (fair) information when comparing the computation of the two constructions.

In this sense, this thesis also employs algorithms based on Reiter's Hitting Set Tree (HST) [Rei87]. Many studies exist providing different heuristics and optimisations of this algorithm in Ontology Repair and Belief Change [Was00, SQJH08, Moo10, Hor11, CW15]. Note that there are works which do not rely on Hitting Set algorithms, a recent example is due to Ji, Boutouhami and Qi [JBQ19] that uses integer linear programming instead.

More specifically, this thesis considers the variant described in Algorithm 1. This version includes optimisations such as node reuse, early path termination and guarantees that at the end, the paths returned are $\subseteq$-minimal [Hor11, Cób14]. The nodes can be either MaxNons or MinImps, the set of closed paths indicates the minimal sets for which no node was found. The set of closed paths are particularly useful for MinImps-based approaches as they correspond to the $\subseteq$-minimal incisions.

For clarity, instead of calling the reasoner directly, it is assumed the existence of an "oracle". This oracle can encode any monotonic property (w.r.t set inclusion), and the algorithms would still work. For this thesis, the oracle implements only the method "entails" which indicates whether a set $X$ is such that $\operatorname{Cn}(X \cup \Phi) \cap \Omega$, where $\Omega$ and $\Phi$ are determined beforehand and correspond to the sets of unwanted and desired formulas, respectively.

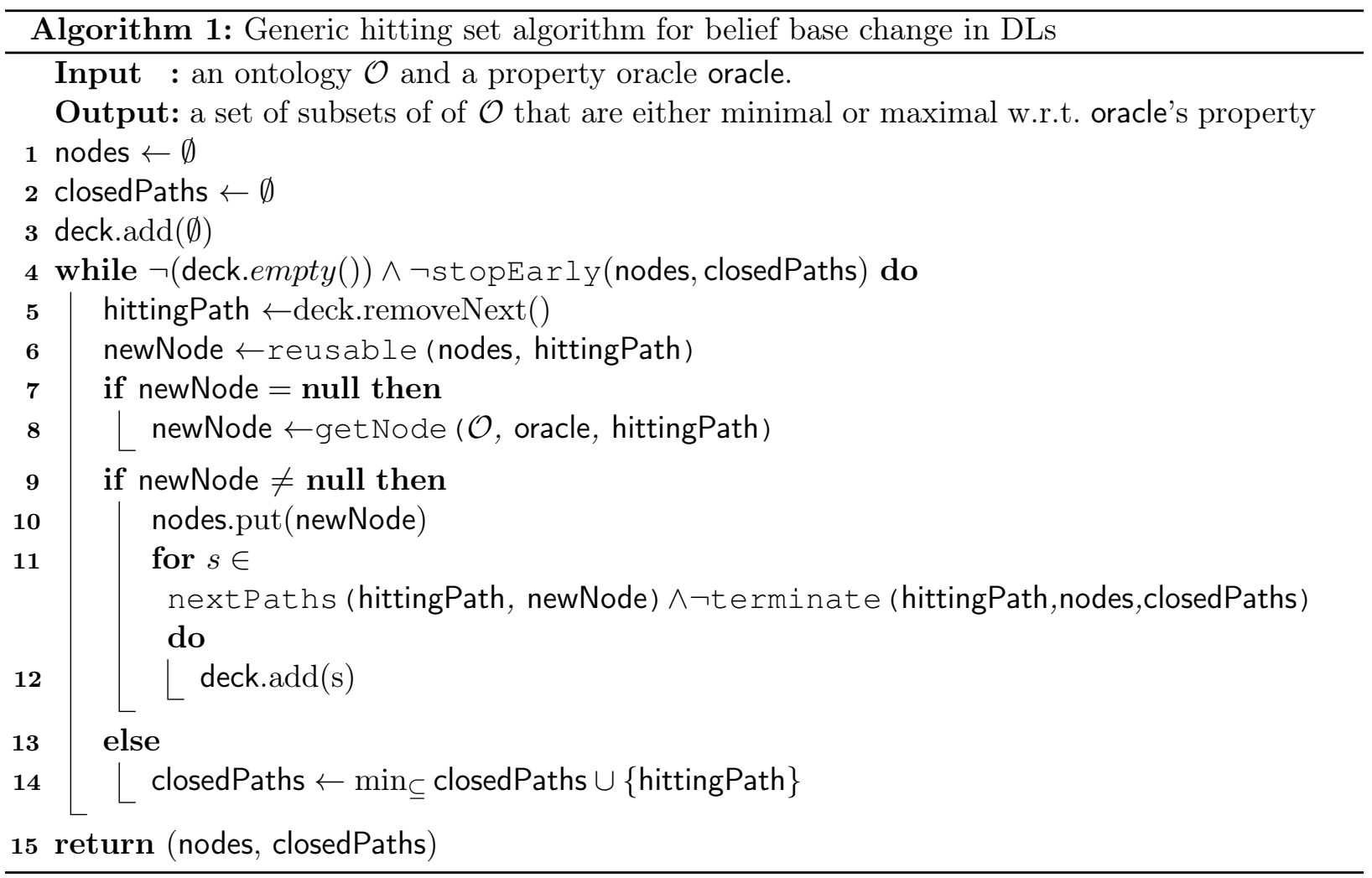

The function getNode returns a set (MinImp or MaxNon) according to the current path being analysed. The method nextPaths generates the successors to be explored from a certain node, terminate checks whether a path can be pruned early, and stopEarly is used to stop the computation when only partial results are sufficient. As for the navigation, the algorithm uses an arbitrary structure which only implements three methods: check for emptiness, add an element and remove an element. The removal order determines how the tree is built, e.g. via depth-first search or breadth-first search.

The functions getNode, nextPaths and reusable are determined according to which set is being computed. Algorithm 2 shows their implementation for MinImps, Algorithm 3 for MaxNons.

As for the functions anyMin Imp and anyMaxNon Horridge [Hor11], Ribeiro [Rib13], and Cóbe and Wassermann [CW15] also employ black-box methods which use an arbitrary reasoner which must only be able to answer whether a set of formulas $Y$ is a subset of the consequences of a set 

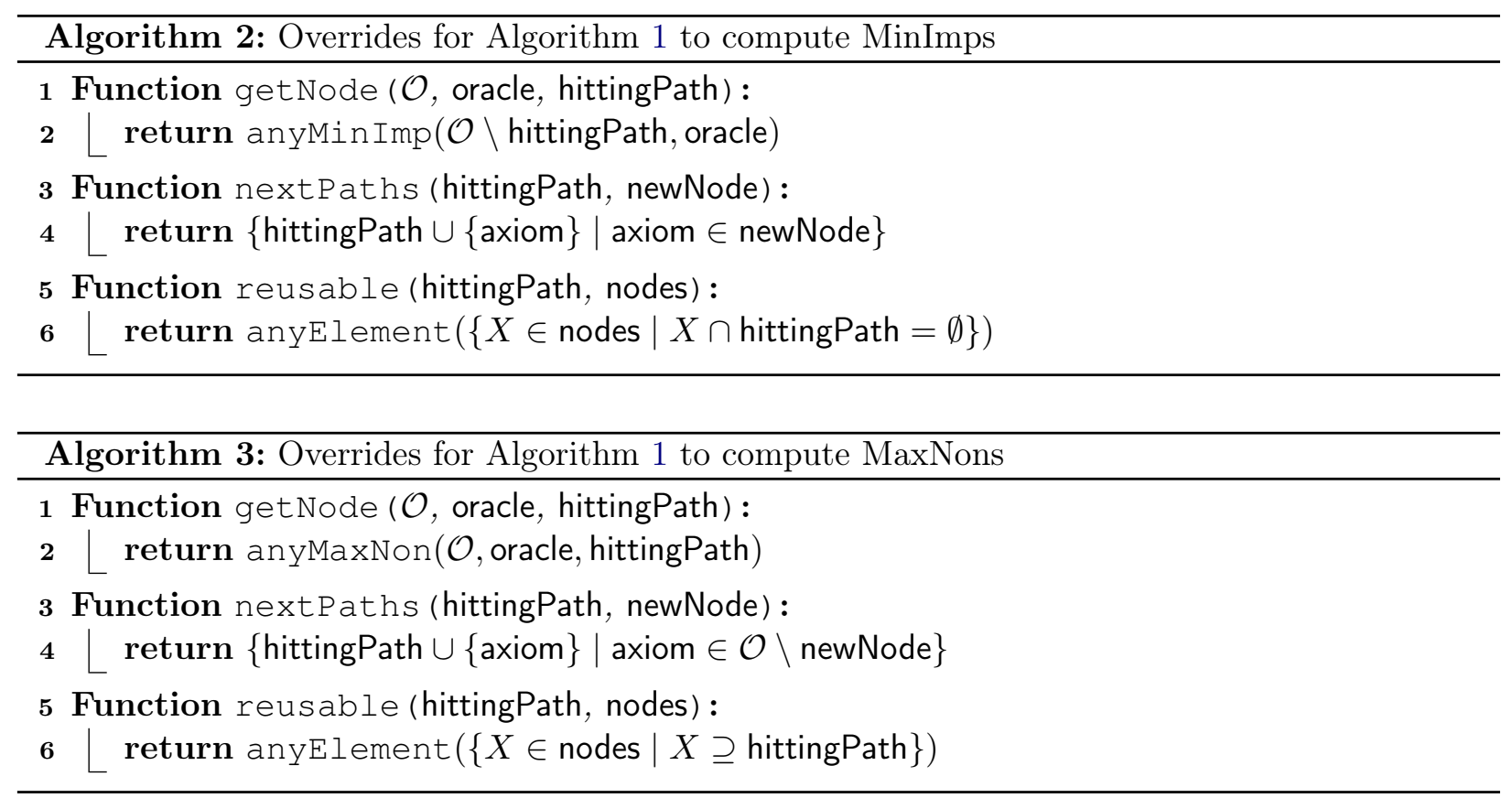

of formulas $X$ (that is, to answer $X \subseteq C n(Y)$ ). The algorithm for computing MinImps enlarges a set until it entails $Y \subseteq \operatorname{Cn}(X)$, then shrinks it by removing unnecessary axioms. For MaxNons, the algorithm shrinks the ontology first getting rid of the unwanted entailment, then it recovers as many axioms as possible. These overarching methods are depicted in Algorithms 4 and 5.

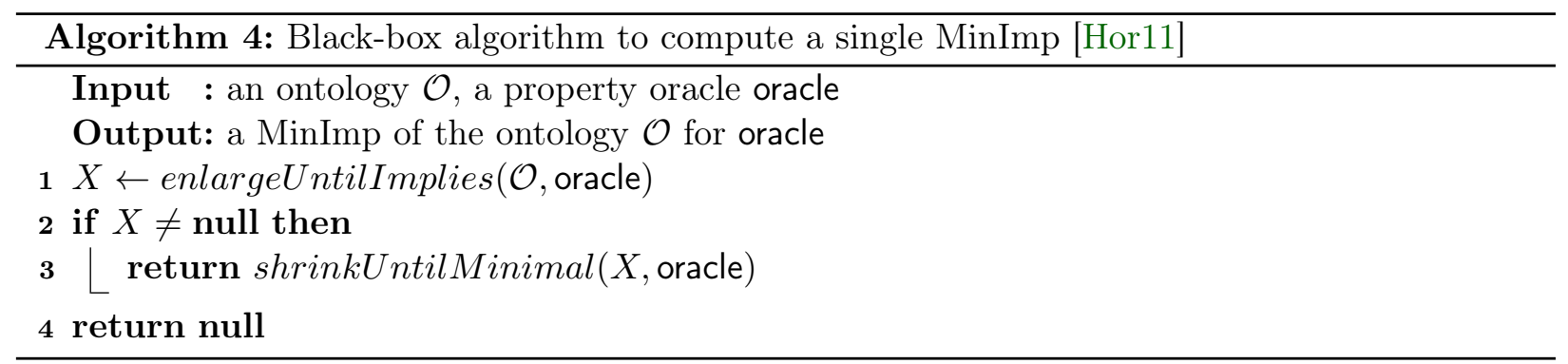

There are many ways to implement the enlargement and shrinking methods for both MinImps and MaxNons. Due to time constraints, instead of comparing all of them when performing the experiments, this thesis relies on the works of Horridge [Hor11] and Cóbe and Wassermann [CW15]. These studies discuss the best heuristics for MinImps and MaxNons, Cobe and Wassermann [Cób14, CW15] even compare then empirically. The next algorithms in this subsection describe the enlargement and shrinking strategies considered.

Algorithm 6 is the simplest method to obtain an underapproximation of a MinImp: the whole ontology can be return as long as it satisfies oracle's criterion. Otherwise, due to monotonicity, there is no MinImp.

Horridge [Hor11] and Cóbe and Wassermann [Cób14, CW15] employ the notion of syntactic relevance or syntactic connectivity in different ways. Cóbe and Wassermann [CW15] make a single pass over the axioms selecting those connected to the input signature, while Horridge implements a strategy that evaluates each axiom differently and iteratively increases the signature with new terms, and thus new axioms.

Instead of choosing one of these approaches, a compromise was made. The strategy devised by Cóbe and Wassermann [CW15] is very simplistic and gets only the immediately relevant axioms, while Horridge's becomes too close to what certain modularity approaches do (as some discussed in Section 2.4) and is much more complex to implement [Hor11]. Algorithm 7 displays the resulting method. 

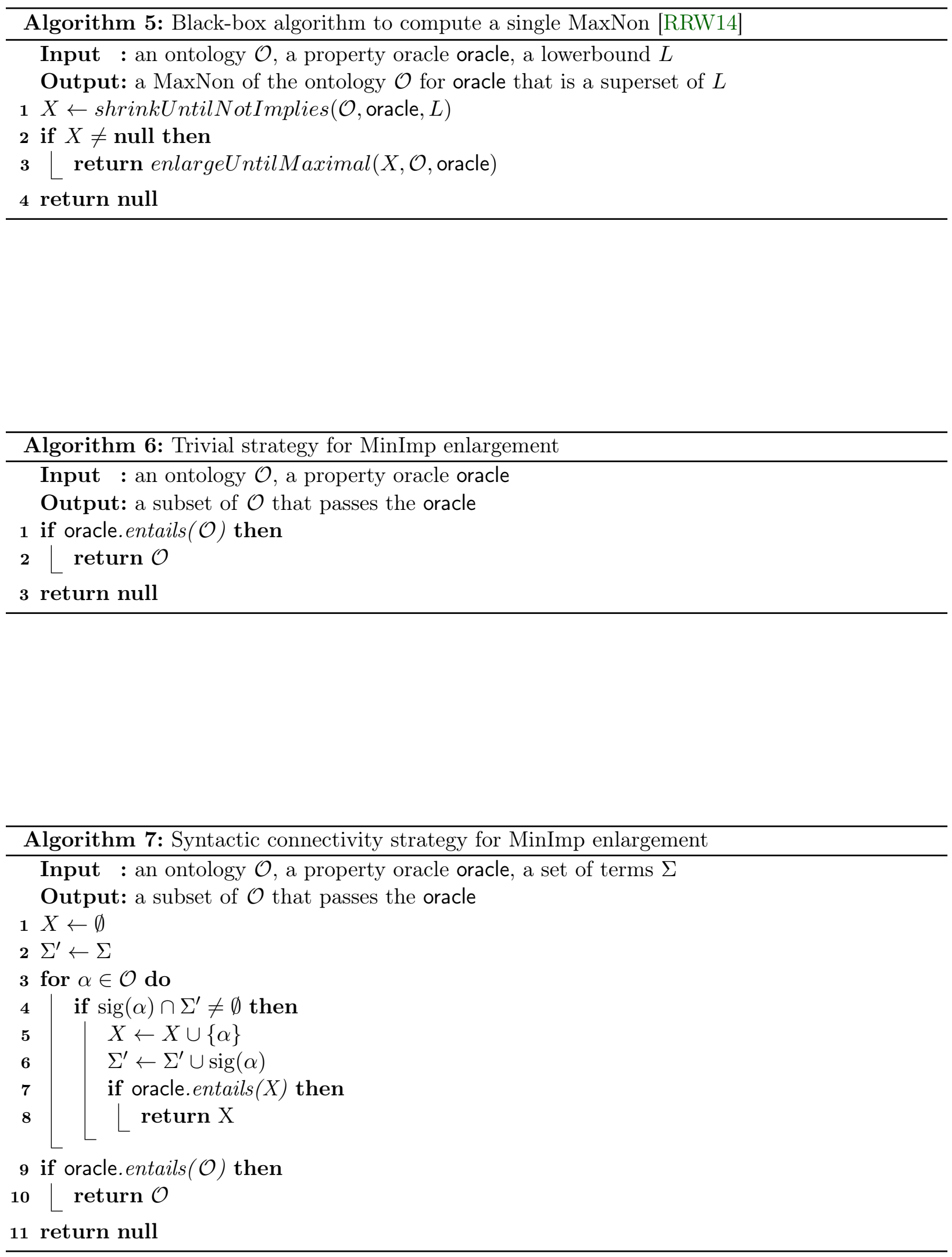
Algorithm 8 shows a strategy proposed by Junker [Jun01] and studied by Horridge [Hor11] and Cóbe and Wassermann [CW15] to shrink MinImps. It splits the base trying to find a MinImp, recombining the parts when it fails to do so.
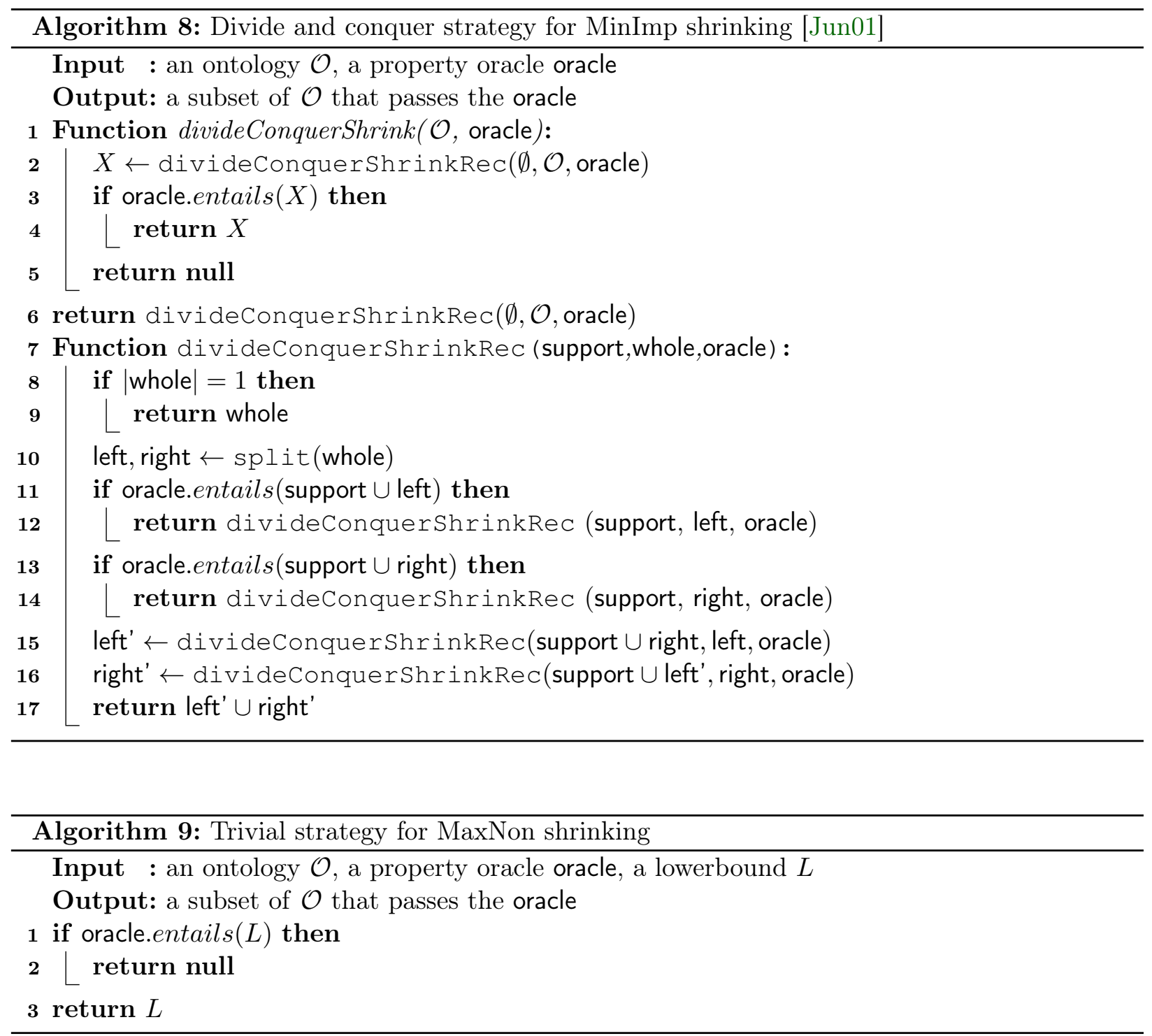

One of the simplest ways to shrink a set until it does not imply a formula is to take the empty set (it works as long as the formula is not tautological). In this direction, Algorithm 9 shows the trivial MaxNon shrinking, which returns the lower bound as long as it does not satisfy the oracle. If even the lower bound passes, there is none of its supersets that is a MaxNon (because the oracle is monotonic).

Algorithm 10 is the dual of Algorithm 7. It has analogous modifications over Cóbe and Wassermann's implementation [CW15].

Finally, Algorithm 11 shows the iterative method to enlarge a MaxNon. It is very simply as the axioms are evaluated one by one, being added as long as the set remains non-implying.

\subsection{Modularity and Logic}

This section briefly discusses the topic of modularity in Logic, in particular, in Belief Change. Further, it also introduces the theme of Ontology Modularity and presents the modules used in the theoretical and empirical contributions. 

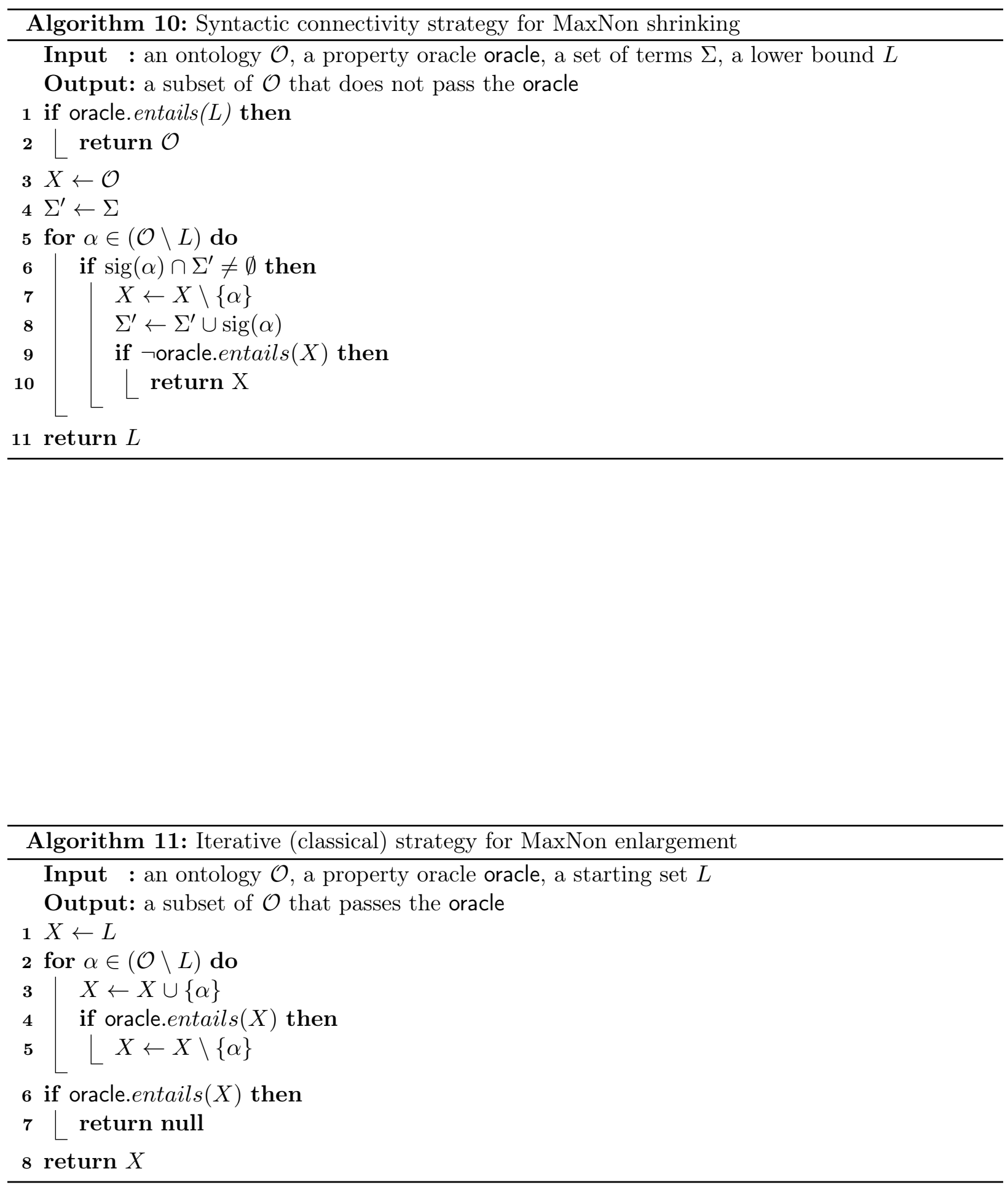
In this section, the term modularity refers to the approaches that, given a set of formulas, try to select relevant subsets for a specific application, or the ones that use some criteria to structure the set of formulas. Note that while this definition includes manual approaches, such as methodologies to develop modular knowledge bases [PS09], this thesis focuses on the automatic (or at least semiautomatic) approaches, particularly those based in Logic.

One of the earliest characterisations of the term "module" in the sense proposed in this thesis is due to Garson [Gar89]. The mentioned definition, which was defined for theories (e.g. belief sets), required not only that the module did not introduce entailments that were not in the original theory and that it must be complete with respect to its signature, i.e., it had all entailments that the original theory had about the terms it included. Already in that study, two prominent motivations for modules become clear: improve computational efficient of algorithms and aid comprehension by exposing a meaningful structure within the theory. About two decades later these criteria would reappear, at least in intuition, in the modularity formalisations proposed by Konev et al. [KLWW09] and by Kontchakov et al. [KPS $\left.{ }^{+} 09\right]$.

One of the earliest characterisations of the term "module" in this sense is due to Garson [Gar89]. Garson defines that modules for theories (e.g. belief sets) must be locally correct and locally complete. A module is locally correct if it does not introduce entailments that were not in the original theory. A module is locally complete if it has all entailments that the original theory had about the terms it includes. Already in that study, two prominent motivations for modules become clear: improve the computational performance of algorithms and aid comprehension by exposing a meaningful structure within the theory. About two decades later the intuitive idea behind these criteria would reappear in the modularity formalisations proposed criteria would reappear, at least in intuition, in the modularity formalisations proposed by Konev et al. [KLWW09] and Kontchakov et al. $\left[\mathrm{KPS}^{+}\right.$09].

In Belief Change, the Splitting Languages [Par99] is one of the first approaches regarding modularity. Parikh's motivation with this model is to address one particular issue with the traditional AGM postulates, which allows what he calls a "trivial update". The trivial update is simply a revision operation that, upon receiving an input that conflicts with its current epistemic state, discards everything it had before. The approach to avoid that is to contain a revision to only a part of the belief set. The intuitive idea is to partition the formulas into connected components determined by the terms shared in the signature. Then, the revision is performed only considering the partitions whose signature intersects that of the input.

Even though the idea of using the signature as a tool to split theories and knowledge bases usually leads to large modules, it comes again in more recent modularity approaches [KLPW10].

Other approaches in modularity appeared in Belief Change [CP00, HW02, Par09], often with the concept of logical relevance [KM07, Mak09, PWCF15] which essentially states that one formula is relevant to another if either aids or hinders its proof. Among these, the most pertinent of these proposals to this thesis is the theory Local Change theory [HW02], discussed in subsection.

\subsubsection{Local Change}

The Local Change theory proposed by Hansson and Wassermann [HW02] proposes the use of sets called compartments as a way to exclude irrelevant axioms when performing Belief Change operations. These sets are obtained via a function that returns the union of the consistent MinImps of a base for a given input formula, as Definition 2.4.1.

Definition 2.4.1 (Compartmentalization Function [HW02]). Let Cn be an inference operation. The function cpart is the compartmentalization function based on $\mathrm{Cn}$ if, for all $B, \Phi \subseteq \mathfrak{L}$ : $\operatorname{cpart}(\Phi, B)=$ $\bigcup_{\varphi \in \Phi} \operatorname{cpart}(\varphi, B)$, where 


$$
\operatorname{cpart}(\varphi, B)= \begin{cases}\emptyset & \text { if } \varphi \in \operatorname{Cn}(\emptyset) \text { or } \neg \varphi \in \operatorname{Cn}(\emptyset) \\ \backslash \operatorname{MinImps}(B, \emptyset,\{\perp\}))) & \text { otherwise }\end{cases}
$$

Also, we refer to $\operatorname{cpart}(\varphi, B)$ as the $\varphi$-compartment of $B$ and to $\operatorname{cpart}(\Phi, B)$ as the $\Phi$-compartment of $B$.

However, there are two issues with compartments. The first is that it relies on computing the whole MinImps (kernels) for a given formula, which is computationally expensive. The second is the dependency on the classical negation of sentences, which is not well-defined in most description logics (for instance, there is no clear meaning for a negation of a concept inclusion such as $\mathrm{A} \sqsubseteq \mathrm{B}$ ).

Furthermore, the compartments might not be sufficient for repairs, since they exclude formulas from the inconsistent MinImps. Still, the definition of compartments relates to modularity via the criterion of local correctness proposed by Garson [Gar89].

As such, the compartments are more suitable to identify relevant formulas for a given input, as their usage for debugging and repairing ontologies is compromised. Still, it is possible to define consequence relations (in the same sense as $\mathrm{Cn}$ ) based on compartments. The inference methods preserve desirable properties from the consequence used in Definition 2.4.1 such as monotonicity and compactness. These localised consequence relations can be employed in contraction and revision functions that satisfy a set of postulates, although weaker ones when compared to the classical approach for Belief Bases [HW02, SMRW18].

Moreover, the Local Change theory provides a construction that is able to identify which axioms are relevant for an entailment. By effect, extracting compartments can help in mitigating the multiple outcome problem, as axioms that do not concern the entailment are completely ignored. This idea was be explored briefly by Guimarães and Wassermann [GW17].

Furthermore, the results in Local Change theory highlight the importance of pinpointing the MinImps for Belief Change, an argument that will underpin our choice of modules later in this chapter.

\subsubsection{Modularity in Ontologies}

In the realm of ontologies and description logics, applications of modules include classification [Sun08, CHWKS10, Mat16], Ontology Debug and Repair [SQJH08, BS08, Moo10, Hor11], Ontology Comprehension [CPSK06, DPSS11] and meta-reasoning heuristics [RCH12, TP12].

Considering the area of Ontology Modularity as whole, there are a number of approaches which differ on whether they concern the design and import of ontologies in a modular way, extracting a sufficient part of the ontology for an application, or aiding users to understand the organisation and relationship between the terms and axioms in the ontology [PS09]. Moreover, the modularity techniques also vary on whether the means to obtain the module, for instance: syntactical analysis, reasoning, graph navigation or statistical methods [PS09].

For this thesis, the first limit on scope is to focus solely on modules tailored for extraction of sufficient parts for reasoning-based applications. Among the techniques that fit into this description are $\mathcal{E}$-connections [CPSK06], Conservativity-based Modules (CBMs) [KLWW09], Locality-based modules (LBMs) [CHKS08], Reachability-based modules (RBMs) [Sun09], Minimal Depleting QueryInseparability modules (MDQMs) [KPS ${ }^{+}$09, KWZ10], $\Delta$-decomposition [KLPW10], the modules from AMEX system [GKW14], Lean-kernels [PMIMS17], best excerpts [CLMW17] and others. Del Vescovo [Del13] compares many of these approaches regarding their ability to represent the modular structure of an ontology concisely and in a meaningful way. This topic will be revisited later in Subsection 2.4.4.

However, not every approach to modularity in ontologies, even the ones founded in Logic, is suitable for integration with Belief Base Change and Ontology Repair. Another requirement imposed 
in this thesis is that the modules produced must be justification-preserving, as in Definition 2.4.2, which is an adaptation of previous definitions by Suntisrivaraporn et al. [SQJH08] and Du, Qi and Ji [DQJ09].

Definition 2.4.2 (Justification-Preserving Sets). Let $\mathcal{O}, A$ be sets of formulas. A set $X$ is:

1. justification-preserving for $\mathcal{O}$ and $A$ : if there is a surjective function $j$ such that

$$
j: \operatorname{MinImps}(X, A, \emptyset) \rightarrow \operatorname{MinImps}(\mathcal{O}, A, \emptyset) .
$$

2. a justification-preserving module (or JPM, for short) of $\mathcal{O}$ for $A$ if $\mathcal{O}^{\prime}$ it is a justificationpreserving subset of $\mathcal{O}$. An arbitrary justification-preserving module of $\mathcal{O}$ for $A$ is expressed as $\bmod (\mathcal{O}, \Omega)$.

Note that in Definition 2.4.2, the MinImps with the empty set as third argument corresponds to the $A$-kernel set of $\mathcal{O}$, i.e., the (root) justifications of $\mathcal{O}$ for $A$ [MGSW19]. Proposition 4.1.1 in Chapter 4 shows that with minor adjustments JPMs can also preserve MinImps in general.

There are already studies in the literature which acknowledge the importance of justification-preserving modules to improve ontology debug and repair approaches [SQJH08, DQJ09, RKCH16]. For instance, Suntisrivaraporn et al. [SQJH08] and Horridge [Hor11] use these modules (more specifically the locality-based modules [CHKS08]) to improve performance of MinImps computation.

Both semantic and syntactic variants of the LBMs, $\mathcal{E}$-connections and $\Delta$-decomposition are examples of strategies that generate JPMs as per Definition 2.4.2.

However, many existing and notable modularity approaches do not fit the definition of JPM proposed. Best excerpts [CLMW17] are not JPMs because they only guarantee that one MinImp is included. RBMs for $\mathcal{S R \mathcal { I } Q}$ [NBM13] and Lean-kernels [PMIMS17] are examples of strategies that produce justification-preserving sets. These latter two do not yield modules according to Definition 2.4.2 because they transform the original ontology losing the subset characteristic (and with no simple way to obtain the original MinImps).

Even so, it is important to note that general justification-preserving sets could be useful for Pseudo-Belief Change. This subarea of Belief Change replaces the inclusion postulate, which forbids normalisations, by a more general one, called logical inclusion, that only constrains the logical closure of the result. Another possible usage, concerns the techniques such as the best excerpts, that although not justification-preserving, could still be employed as expansion strategy when computing a single MinImp as in Algorithm 4.

\subsubsection{Syntactic Locality-Based Modules}

For most of the theoretical results proved in this thesis, it is sufficient to consider an arbitrary JPM strategy. However, for the empirical pursuits and to obtain some additional results, additional properties are required from the modularity approaches. One of them is polynomial extraction since the modules must not be a bottleneck when computing MinImps and MaxNons. Another important criterion is that the module should be small enough to be useful in empirical applications.

The next requisite involves the use of stratification and the modular structure to mitigate the multiple outcome problem. It corresponds to the ability to derive a meaningful structure using such modules, that later can be employed to select among axioms or MaxNons via selection functions. Moreover, such a structure must be computed efficiently.

The last condition is pragmatic: favouring modularity approaches that can be easily integrated into implementations via an API. It is even better if this modularity approach has already been studied in different empirical settings.

The syntactic locality-based modules [CHKS08] (onwards, simply locality-based modules or LBMs) satisfy all of the conditions imposed. They are justification-preserving and work for any DL up to $\mathcal{S R O I} \mathcal{I}(\mathcal{D})$, which is the most expressive logic considered in this thesis [Del13]. Also, they can be extracted in polynomial time on the size of the ontology and its signature [CHKS08, Tsa12]. 
Further, they are good approximations of their semantic counterparts $\left[\mathrm{DKP}^{+} 12\right]$ and have been employed different of studies, including some related to Ontology Repair [SQJH08, Hor 11]. Moreover, they comply with our pragmatic requisite: these modules are implemented in the OWL $\mathrm{API}^{2}$, which is already a dependency on the implementation of the Belief Change operations used in this study.

The LBMs meet the requirement on the modular structure via the atomic decomposition, a method that is covered later in Subsection 2.4.4. The structure obtained is both concise, it has a meaningful interpretation, and it can be computed in polynomial time.

The extraction of locality-based modules needs an ontology $\mathcal{O}$ and an input signature $\Sigma$. The resulting module is a JPM of the ontology $\mathcal{O}$ for any finite set $\Phi$ of DL formulas such that $\operatorname{sig}(\Phi) \subseteq \Sigma$. The intuition behind the extraction procedure is to detect which axioms do not constrain the interpretation of terms in $\Sigma$. Roughly speaking, the algorithm replaces terms not in $\Sigma$ by $\top$ or $\perp$ (the choice of which defines the type of locality) in every axiom, and then only adding to the module the ones that did not become tautologies. At each iteration the new axioms bring new terms to the signature and the process is repeated until a fixed point is reached. The iteration until this fixed point guarantees properties that lead to justification-preservation (such as self-containment which will be defined posteriorly).

Definition 2.4.3 presents syntactic locality formally for the DL $\mathcal{S R O \mathcal { I }}$. Note that the definition that has two parts, the first which describes the "substitution" and the second that regards the detection axioms that become tautologies after the replacement.

Definition 2.4.3 (Syntactic Locality [CHKS08, SSZ09, Del13]). Let $\Sigma$ be a signature, $N_{C}, N_{R}$ and $N_{I}$ partitions of the vocabulary as discussed in Section 2.2. For each concept name A $\in N_{C} \backslash \Sigma$ consider two aliases: $\mathrm{A}^{\perp}$ for $\perp$-locality and $\mathrm{A}^{\top}$ for $T$-locality. Assume the analogous for concept names in $N_{R} \backslash \Sigma$.

With these aliases, consider the sets $\operatorname{Top}(\Sigma)$ and $\operatorname{Bot}(\Sigma)$ as in Figure 2.2:

1. $\perp$-locality (Let $\left.\mathrm{A}^{\perp}, \mathrm{r}^{\perp} \notin \Sigma, \mathrm{C}^{\perp} \in \operatorname{Bot}(\Sigma), \mathrm{C}_{(i)}^{\top} \in \operatorname{Top}(\Sigma), n>0 \in \mathbb{N}\right)$

$\operatorname{Bot}(\Sigma) \quad::=\perp\left|\mathrm{A}^{\perp}\right| \mathrm{r}^{\perp} . \mathrm{C}\left|\neg \mathrm{C}^{\top}\right| \mathrm{C} \sqcap \mathrm{C}^{\perp}\left|\mathrm{C}^{\perp} \sqcap \mathrm{C}\right| \exists \mathrm{r} . \mathrm{C}^{\perp}\left|\leq n \mathrm{r} . \mathrm{C}^{\perp}\right| \exists \mathrm{r}^{\perp} . \mathrm{C} \mid \leq n \mathrm{r}^{\perp} . \mathrm{C}$

$\operatorname{Top}(\Sigma) \quad::=\top\left|\neg \mathrm{C}^{\perp}\right| \mathrm{C}_{1}^{\top} \sqcap \mathrm{C}_{2}^{\top} \mid \leq 0$ r.C

2. T-locality (Let $\mathrm{A}^{\top}, \mathrm{r}^{\top} \notin \Sigma, \mathrm{C}^{\perp} \in \operatorname{Bot}(\Sigma), \mathrm{C}_{(i)}^{\top} \in \operatorname{Top}(\Sigma), n>0 \in \mathbb{N}$ )

$\operatorname{Bot}(\Sigma) \quad:=\perp\left|\neg \mathrm{C}^{\top}\right| \mathrm{C} \sqcap \mathrm{C}^{\perp}\left|\mathrm{C}^{\perp} \sqcap \mathrm{C}\right| \exists \mathrm{r} . \mathrm{C}^{\perp} \mid \leq n$ r. $\mathrm{C}^{\perp}$

$\operatorname{Top}(\Sigma) \quad::=\top\left|\mathrm{A}^{\top}\right| \mathrm{r}^{\top} . \mathrm{C}\left|\neg \mathrm{C}^{\perp}\right| \mathrm{C}_{1}^{\top} \sqcap \mathrm{C}_{2}^{\top}\left|\exists \mathrm{r}^{\top} . \mathrm{C}^{\top}\right| \leq n \mathrm{r}^{\top} . \mathrm{C}^{\top} \mid \leq 0$ r.C

Figure 2.2: Syntactic locality conditions

An axiom $\alpha$ is syntactically $\perp$-local w.r.t. signature $\Sigma$ if it is of the form: $\mathrm{C}^{\perp} \sqsubseteq \mathrm{C}, \mathrm{C} \sqsubseteq \mathrm{C}^{\top}$, $\mathrm{C}^{\perp} \equiv \mathrm{C}^{\perp}, \mathrm{C}^{\top} \equiv \mathrm{C}^{\top}, \mathrm{r}^{\perp} \sqsubseteq \mathrm{r}, \operatorname{Trans}\left(\mathrm{r}^{\perp}\right)$ or $\operatorname{Func}\left(\mathrm{r}^{\perp}\right)$.

An axiom $\alpha$ is syntactically $\top$-local w.r.t. signature $\Sigma$ if it is of the form: $\mathrm{C}^{\perp} \sqsubseteq \mathrm{C}, \mathrm{C} \sqsubseteq \mathrm{C}^{\top}$, $\mathrm{C}^{\perp} \equiv \mathrm{C}^{\perp}, \mathrm{C}^{\top} \equiv \mathrm{C}^{\top}, \mathrm{r} \sqsubseteq \mathrm{r}^{\top}, \operatorname{Trans}\left(\mathrm{r}^{\top}\right), \operatorname{Func}\left(\mathrm{r}^{\top}\right)$ or $\mathrm{C}^{\top}(a)$.

The definition LBMs, depends on following Algorithm 12 which uses the notion of syntactic locality of Definition 2.4.3.

Finally, the syntactic LBMs are defined as follows:

Definition 2.4.4 (Locality-Based Modules [CHKS08, SSZ09]). Let $\mathcal{O}$ be an ontology, $\Sigma$ a signature, $x$ and $y$ locality notions in $\{\top, \perp\}$ such that $x \neq y$. Then:

1. An ontology $\mathcal{M}$ is the $x$-locality-based module of $\mathcal{O}$ w.r.t. $\Sigma$, in symbols $\mathcal{M}=x-\operatorname{LBM}(\mathcal{O}, \Sigma)$, if it is the output of Algorithm 12 when given as input $\mathcal{O}, \Sigma$ and $x$.

\footnotetext{
${ }^{2}$ https://owlcs.github.io/owlapi/
} 


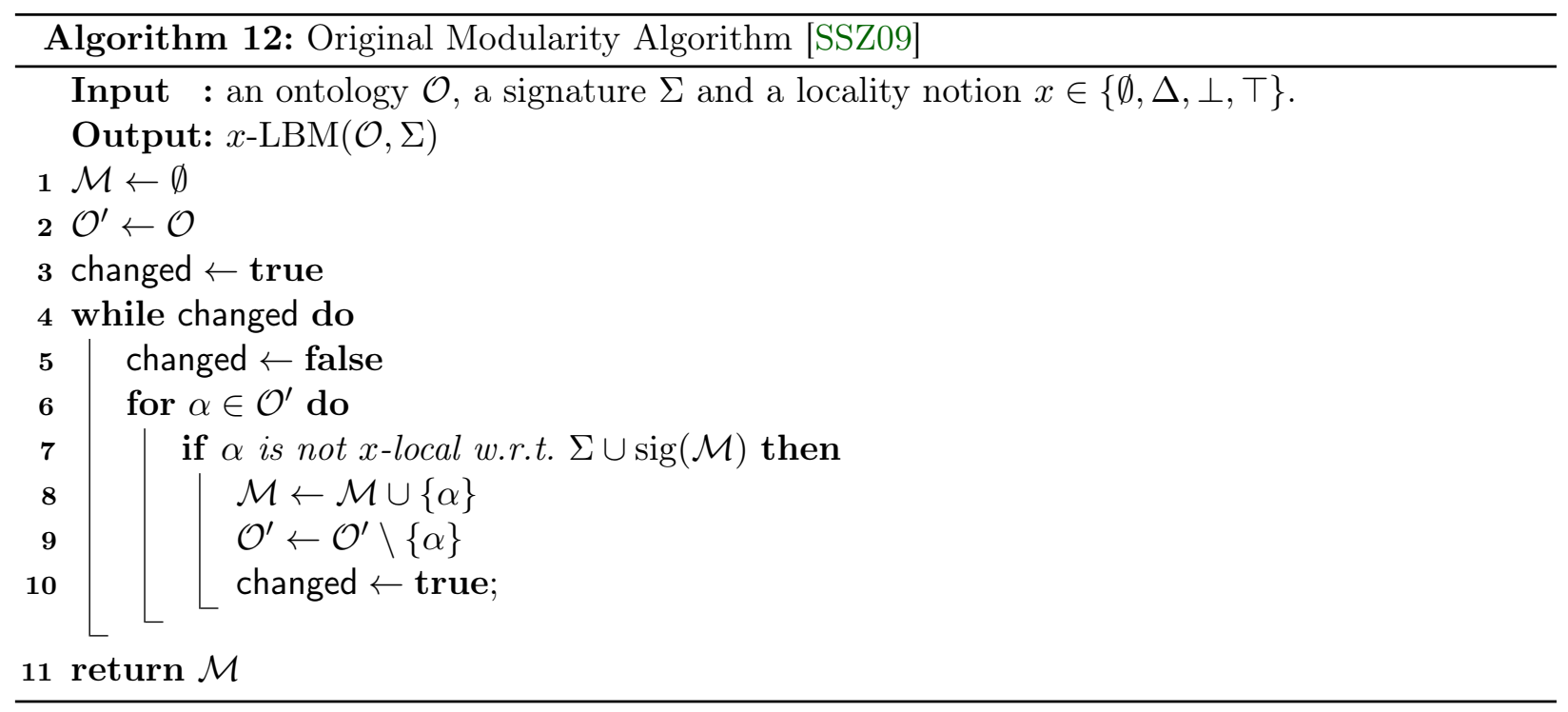

2. An ontology $\mathcal{M}$ is the $x y$-locality-based module of $\mathcal{O}$ w.r.t. $\Sigma$, in symbols $\mathcal{M}=x y$ - $\operatorname{LBM}(\mathcal{O}, \Sigma)$, if $\mathcal{M}=x-\operatorname{LBM}(y-\operatorname{LBM}(\mathcal{O}, \Sigma), \Sigma)$.

3. Let $\left(\mathcal{M}_{i}\right)_{i \geq 0}$ a sequence of ontologies such that $\mathcal{M}_{0}=\mathcal{O}$ and $\mathcal{M}_{i+1}=x y-\operatorname{LBM}\left(\mathcal{M}_{i}, \Sigma\right)$ for every $i \geq 0$. For the smallest $n \geq 0$ with $\mathcal{M}_{n}=\mathcal{M}_{n+1}$, we call $\mathcal{M}_{n}$ the $x y^{*}$-locality-based module of $\mathcal{O}$ w.r.t. $\Sigma$, written $\mathcal{M}=x y^{*}-\operatorname{LBM}(\mathcal{O}, \Sigma)$.

Interestingly, $\top \perp^{*}$-LBMs and $\perp \top^{*}$-LBMs coincide [Del13].

To evaluate the complexity of LBM extraction, let $n$ be the number of axioms in an ontology, $l=$ $\max _{\alpha \in \mathcal{O}} \ell(\alpha)$, where $\ell(\alpha)$ denotes the number of term constructors or axiom symbols in $\alpha$ and $s=$ $\max _{\alpha \in \mathcal{O}}|\operatorname{sig}(\alpha)|$. Also, assume that the number of locality-rules is fixed. Without loss of generality one can consider only those from Definition 2.4.3), which has a constant amount of rules. Then, testing an axiom $\alpha$ for syntactic locality ( $\top$ or $\perp$ ), takes time $O(\ell(\alpha)$ ). Therefore, Algorithm 12 takes time $O\left(n^{2} \cdot l\right)$ [Tsa12]. Tsarkov proposes an improved version whose time complexity for extraction $\top$ and $\perp$-LBMs is in $O(n \cdot s \cdot l)$. Additionally, the fixed point version of the LBMs $\left(\top \perp^{*}\right)$ requires at most $n$ module extractions.

Example 2.4.5 illustrates how modules can aid in the computation of MinImps and MaxNons.

Example 2.4.5. Consider the ontology MovieEx from Example 2.2.1. Now, suppose the user needs to add the information that a violent teaser has a violent scene, that is,

\section{$\varphi:$ ViolentTeaser $\sqsubseteq \exists$ hasScene.ViolentScene.}

The addition of $\varphi$ to MovieEx makes ViolentTeaser unsatisfiable. To identify the root causes one can extract, for example, the $\perp$-LBM for the signature $\{$ ViolentTeaser $\}$ :

$$
\perp-\operatorname{LBM}(\text { MovieEx } \cup\{\varphi\},\{\text { ViolentTeaser }\})=\left\{\varphi, \alpha_{1}, \alpha_{2}, \alpha_{6}, \alpha_{7}, \alpha_{8}\right\} .
$$

The module is closer to the MinImp (justification): $\left\{\varphi, \alpha_{1}, \alpha_{2}, \alpha_{6}, \alpha_{7}, \alpha_{8}\right\}$.

Now, some properties of the LBMs are discussed to make this thesis self-contained and justify the focus on LBMs. However, before presenting them, some intermediate concepts are needed. One of them is the notion of inseparability relation shown in Definition 2.4.6. These relations are used to define and prove properties of modularity strategies such as CBMs [KLWW09], semantic and syntactic LBMs [SSZ09], RBMs [NBM13] and others [KPS ${ }^{+}$09, RKCH16].

Definition 2.4.6 (Inseparability [KLWW09, SSZ09]). Let $\mathfrak{L}$ be a language and let $S$ be a family of equivalence relations defined as: 


$$
S=\left\{\equiv \sum_{\Sigma}^{S} \mid \Sigma \text { is a signature }\right\}
$$

Each relation in $S$ is called an inseparability relation. Two ontologies in an inseparability relation in $S$ w.r.t. a signature $\Sigma$ are said to be $S$-inseparable w.r.t. $\Sigma$.

In Definition 2.4.6 above, the meaning of $S$ varies according to the application. Two of the most common families are deductive and model inseparability. By deductive inseparability, two ontologies $\mathcal{O}$ and $\mathcal{O}^{\prime}$ in $\mathfrak{L}$ are inseparable w.r.t. $\Sigma$ if $\operatorname{Cn}(\mathcal{O}) \cap\{\varphi \in \mathfrak{L} \mid \operatorname{sig}(\varphi) \subseteq \Sigma\}=\operatorname{Cn}\left(\mathcal{O}^{\prime}\right) \cap\{\varphi \in \mathfrak{L} \mid$ $\operatorname{sig}(\varphi) \subseteq \Sigma\}$. Model inseparability means that the set of interpretations restricted of $\Sigma$ of both ontologies coincide, that is, $\left\{\left.\mathcal{I}\right|_{\Sigma} \mid \mathcal{I} \models \mathcal{O}\right\}=\left\{\left.\mathcal{J}\right|_{\Sigma} \mid \mathcal{J}=\mathcal{O}^{\prime}\right\}$.

Now, the following properties, which are satisfied (not exclusively) by the syntactic LBMs:

Definition 2.4.7 ([KPS $\left.\left.{ }^{+} 09\right]\right)$. Let $S$ be an inseparability relation as in Definition 2.4.6, $\mathcal{O}$ an ontology and $\Sigma$ a signature. A subset $\mathcal{M}$ of $\mathcal{O}$ is called:

1. A $S_{\Sigma}$-module of $\mathcal{O}$ if $\mathcal{M} \equiv{ }_{\Sigma}^{S} \mathcal{O}$.

2. A self-contained $S_{\Sigma \text {-module of } \mathcal{O} \text { if } \mathcal{M} \equiv \stackrel{S}{S} \cup \operatorname{sig}(\mathcal{M})} \mathcal{O}$.

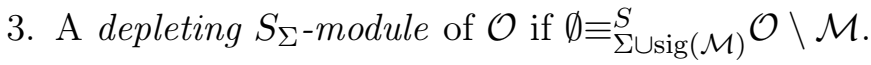

Additionally, a (self-contained, depleting) $S_{\Sigma}$-module $\mathcal{M}$ of $\mathcal{O}$ is called minimal if no proper subset of $\mathcal{M}$ is a (self-contained, depleting) $S_{\Sigma}$-module of $\mathcal{O}$.

Sattler, Schneider and Zakharyaschev [SSZ09] define one inseparability relation for each type of module: two ontologies are inseparable w.r.t. a signature $\Sigma$ if their modules coincide. From these relations, more properties can be inferred. Proposition 2.4.8 presents some useful ones for the proofs in Chapter 4.

Proposition 2.4.8 ([KPS ${ }^{+} 09$, SSZ09]). Let $x$ be a LBM type in $\left\{\top, \perp, \top \perp^{*}\right\}, \mathcal{O}, \mathcal{O}^{\prime}$ ontologies with $\mathcal{O} \subseteq \mathcal{O}^{\prime}$ and $\Sigma, \Sigma^{\prime}$ signatures such that $\Sigma \subseteq \Sigma$. Then:

1. (idempotence) $x-\operatorname{LBM}(\mathcal{O}, \Sigma)=x-\operatorname{LBM}(x-\operatorname{LBM}(\mathcal{O}, \Sigma), \Sigma)$.

2. (monotonicity on ontology enlargements) $x-\operatorname{LBM}(\mathcal{O}, \Sigma) \subseteq x-\operatorname{LBM}\left(\mathcal{O}^{\prime}, \Sigma\right)$.

3. (monotonicity on signature enlargements) $x-\operatorname{LBM}(\mathcal{O}, \Sigma) \subseteq x-\operatorname{LBM}\left(\mathcal{O}, \Sigma^{\prime}\right)$.

Some results proved in this thesis also refer to sets obtained by successive LBM extractions, but that are not modules necessarily. Definition 2.4.9 describes a construction that represents this sequence of extractions.

Definition 2.4.9. Let $\mathcal{O}$ be an ontology, $\Sigma$ a signature, $n \in \mathbb{N}^{*}$, and $x_{i} \in\{\top, \perp\}$ locality notions for $0 \leq i<n$. Then we define the extraction sequence $(x)_{n}-\operatorname{LBM}(\mathcal{O}, \Sigma)$ as:

$$
(x)_{n}-\operatorname{LBM}(\mathcal{O}, \Sigma)= \begin{cases}x_{n}-\operatorname{LBM}(\mathcal{O}, \Sigma) & \text { if } n=1 \\ x_{n}-\operatorname{LBM}\left((x)_{n-1}-\operatorname{LBM}(\mathcal{O}, \Sigma), \Sigma\right) & \text { otherwise }\end{cases}
$$

Note that while every locality-based module is an extraction sequence, the converse does not hold in general, despite the similarities between Definition 2.4.9 and Definition 2.4.4. Extraction sequences make proofs by induction involving $T \perp^{*}$-LBMs simpler. Proposition 2.4.10 lists some properties of extraction sequences (note the similarity with the properties from Proposition 2.4.8).

Proposition 2.4.10. Let $\mathcal{O}, \mathcal{O}^{\prime}$ be ontologies, $\Sigma, \Sigma^{\prime}$ signatures and $(x)_{n}-\operatorname{LBM}(\mathcal{O}, \Sigma)$ an extraction sequence as in Definition 2.4.9. Then,

1. (monotonicity on sequence restriction) If $(y)_{m}$ is a subsequence of $(x)_{n}$, then

$$
(x)_{n}-\operatorname{LBM}(\mathcal{O}, \Sigma) \subseteq(y)_{m}-\operatorname{LBM}(\mathcal{O}, \Sigma) .
$$


2. (monotonicity on ontology enlargements) If $\mathcal{O}^{\prime} \subseteq \mathcal{O}$ then

$$
(x)_{n}-\operatorname{LBM}\left(\mathcal{O}^{\prime}, \Sigma\right) \subseteq(x)_{n}-\operatorname{LBM}(\mathcal{O}, \Sigma) .
$$

3. (monotonicity on signature enlargements) If $\Sigma^{\prime} \subseteq \Sigma$ then

$$
(x)_{n}-\operatorname{LBM}\left(\mathcal{O}, \Sigma^{\prime}\right) \subseteq(x)_{n}-\operatorname{LBM}(\mathcal{O}, \Sigma) .
$$

Proof. They follow directly from properties of the LBMs (Proposition 2.4.8).

Next, Corollary 2.4.11 shows that no extraction sequence will be smaller than the corresponding $\top \perp^{*}$-LBM.

Corollary 2.4.11. Let $\mathcal{O}$ be an ontology, $\Sigma$ a signature and $(x)_{n}-\operatorname{LBM}(\mathcal{O}, \Sigma)$ an extraction sequence with $n \in \mathbb{N}$ as in Definition 2.4.9. Then $\top \perp^{*}-\operatorname{LBM}(\mathcal{O}, \Sigma) \subseteq(x)_{n}-\operatorname{LBM}(\mathcal{O}, \Sigma)$.

Proof. A direct consequence of the definition of $T \perp^{*}$-LBMs (Definition 2.4.4), Proposition 2.4.8(1) and the fact that $x-\operatorname{LBM}(\mathcal{O}, \Sigma) \subseteq \mathcal{O}$ for $x \in\left\{\top, \perp, \top \perp^{*}\right\}$.

Remarks 2.4.12 and 2.4.13 are direct consequences of the properties of modules studied by Cuenca Grau et al. [CHKS08] and Sattler, Schneider and Zakharyaschev [SSZ09]. However, they make demonstrations cleaner and facilitate their understanding (even more for readers who are not familiar with LBMs).

Remark 2.4.12. Let $\mathcal{O}$ be an ontology $\Sigma$ a signature and $x \in\{\perp, \top\}$ a locality type. Then, $x-\operatorname{LBM}(\mathcal{O}, \Sigma)=\{\alpha \in \mathcal{O} \mid \alpha$ is non- $x$-local w.r.t. $\Sigma \cup \operatorname{sig}(x-\operatorname{LBM}(\mathcal{O}, \Sigma))\}$.

Proof. Since $x-\operatorname{LBM}(\mathcal{O}, \Sigma)$ is a self-contained and depleting module, $\mathcal{O} \backslash x-\operatorname{LBM}(\mathcal{O}, \Sigma)$ only has $x$-local axioms w.r.t. $\Sigma \cup \operatorname{sig}(x-\operatorname{LBM}(\mathcal{O}, \Sigma))$. Moreover, these modules are also minimal. Hence they contain no local $x$ axioms for that signature [SSZ09] (otherwise, one could select the subset composed of non- $x$-local ones as the module).

The analogous result for $T \perp^{*}$-LBMs needs only a bit more work.

Remark 2.4.13. Let $\mathcal{O}$ be an ontology, $\Sigma$ a signature. Then:

$\top \perp^{*}-\operatorname{LBM}(\mathcal{O}, \Sigma)=\left\{\alpha \in \mathcal{O} \mid \alpha\right.$ is non- $x$-local for w.r.t. $\Sigma \cup \operatorname{sig}\left(\top \perp^{*}-\operatorname{LBM}(\mathcal{O}, \Sigma)\right)$ for $\left.x \in\{\perp, \top\}\right\}$

Proof. The inclusion of the left-hand side on the right-hand side of Equation (2.1) is a direct consequence of the definition of $T \perp^{*}$-LBMs and self-containment.

For the other direction, let $\alpha$ be as in the right-hand side of Remark 2.4.13. Therefore, as a result of Corollary 2.4.11 and self-containment of $x$-LBMs, $\alpha$ is in any extraction sequence $\left.(x)_{n}-\operatorname{LBM}(\mathcal{O}), \Sigma\right)$, hence $\alpha \in \top \perp^{*}-\operatorname{LBM}(\mathcal{O}, \Sigma)$.

It is also convenient to detach the LBMs from Algorithm 12 to simplify some later proofs. Definition 2.4.14 provides the construction employed for this separation.

Definition 2.4.14. Let $\mathcal{O}$ be an ontology, $\Sigma$ a signature and $x \in\{\perp, \top\}$ a locality-notion. Let $x-\mathrm{NL}_{i}(\mathcal{O}, \Sigma)$, for $i \in \mathbb{N}$, be defined as:

$$
x-\mathrm{NL}_{i}(\mathcal{O}, \Sigma)= \begin{cases}\{\alpha \in \mathcal{O} \mid \alpha \text { is not } x \text {-local w.r.t. } \Sigma\} & \text { if } i=0 \\ \left\{\alpha \in \mathcal{O} \mid \alpha \text { is not } x \text {-local w.r.t. } \Sigma \cup \operatorname{sig}\left(x-\mathrm{NL}_{i-1}(\mathcal{O}, \Sigma)\right)\right\} & \text { if } i>0\end{cases}
$$


It is not sufficient to show Definition 2.4.14; this construction must be linked with the LBMs, and that is precisely the role of Proposition 2.4.17. Before proving this proposition, two other smaller results, namely Remarks 2.4.15 and 2.4.16, are highlighted for clarity.

Remark 2.4.15. Let $\mathcal{O}$ be an ontology, $\Sigma$ a signature and $x \in\{\top, \perp\}$ a locality notion. Since LBMs are never larger than their source ontology for all $i \in \mathbb{N}, x-\mathrm{NL}_{i}(\mathcal{O}, \Sigma) \subseteq x-\mathrm{NL}_{i+1}(\mathcal{O}, \Sigma)$.

Remark 2.4.16. Since $\mathcal{O}$ is finite, as consequence of Remark 2.4.15, there is a $n^{*} \in \mathbb{N}$, such that $x-\mathrm{NL}_{i}(\mathcal{O}, \Sigma)=x-\mathrm{NL}_{n^{*}}(\mathcal{O}, \Sigma), \forall i \geq n^{*}$.

Now, onwards to Proposition 2.4.17.

Proposition 2.4.17. Let $\mathcal{O}$ be an ontology, $\Sigma$ a signature and $x \in\{\perp, \top\}$ a locality-notion. Let $x-\mathrm{NL}_{i}(\mathcal{O}, \Sigma)$ as in Definition 2.4.14. Then $x-\operatorname{LBM}(\mathcal{O}, \Sigma)=x-\mathrm{NL}_{n}^{*}(\mathcal{O}, \Sigma)$ where $n^{*}$ is taken as in Remark 2.4.16.

Proof. Each inclusion has a separate proof:

$x-\mathrm{NL}_{n^{*}} \subseteq x-\operatorname{LBM}(\mathcal{O}, \Sigma)$ : This side applies a proof by induction on $i$ to show that for any $i \in \mathbb{N}$ :

$$
x-\mathrm{NL}_{i}(\mathcal{O}, \Sigma) \subseteq x-\operatorname{LBM}(\mathcal{O}, \Sigma)
$$

Base case: $(i=0)$ Direct consequence of the definition of module.

Induction step: (IH: inclusion holds for $i-1$ ) Let $\alpha \in x-\mathrm{NL}_{i}(\mathcal{O}, \Sigma)$, this means that $\alpha \in \mathcal{O}$ and $\alpha$ is not $x$-local w.r.t. $\Sigma \cup x-\mathrm{NL}_{i-1}(\mathcal{O}, \Sigma)$. Using our induction hyphothesis we obtain $\Sigma \cup \operatorname{sig}\left(x-\mathrm{NL}_{i-1}(\mathcal{O}, \Sigma)\right) \subseteq \Sigma \cup \operatorname{sig}(x-\operatorname{LBM}(\mathcal{O}, \Sigma)$. Therefore, $\alpha \in x-\operatorname{LBM}(\mathcal{O}, \Sigma)$.

Thus $x-\mathrm{NL}_{n^{*}} \subseteq x-\operatorname{LBM}(\mathcal{O}, \Sigma)$.

$x-\operatorname{LBM}(\mathcal{O}, \Sigma) \subseteq x-\mathrm{NL}_{n^{*}}(\mathcal{O}, \Sigma):$ In this case, it is shown by induction that at any execution of Line 8 in Algorithm 12, there will be some $i$ such that $\alpha$ is not $x$-local w.r.t. $\operatorname{sig}\left(x-\mathrm{NL}_{i}(\mathcal{O}, \Sigma)\right)$. In this part of the proof, $\mathcal{M}_{j}$ denotes the value of $\mathcal{M}$ immediately before the $j$-th execution of Line 8. The analagous is done for the corresponding $\alpha: \alpha_{j}$ is the $j$-th axiom added to $\mathcal{M}$ in Algorithm 12.

Base case: $(j=1)$ Before the first execution of Line $8, \mathcal{M}=\emptyset$. Hence, $\alpha_{1}$ is non $x$-local w.r.t. $\Sigma$ and thus $\alpha_{1} \in x-\mathrm{NL}_{0}(\mathcal{O}, \Sigma)$.

Induction step: (IH: for $i<j, \alpha_{i} \in x-\mathrm{NL}_{n^{*}}(\mathcal{O}, \Sigma)$ ) At the $j$-th execution of Line $8, \alpha_{j}$ is not $x$-local w.r.t. $\Sigma \cup \operatorname{sig}\left(\mathcal{M}_{j}\right)$. Using the induction hyphothesis $\mathcal{M}_{j} \subseteq x-\mathrm{NL}_{n^{*}}(\mathcal{O}, \Sigma)$, so $\alpha$ is also non- $x$-local w.r.t. $\Sigma \cup x$ - $\mathrm{NL}_{k}(\mathcal{O}, \Sigma)$, for some $k \in \mathbb{N}$.

Thus, $x-\operatorname{LBM}(\mathcal{O}, \Sigma) \subseteq x-\mathrm{NL}_{n^{*}}$.

Both inclusions together imply the equality $x-\operatorname{LBM}(\mathcal{O}, \Sigma)=x-\mathrm{NL}_{n^{*}}(\mathcal{O}, \Sigma)$.

The last result in this subsection shows that LBMs can be used to repair inconsistent ontologies. Moodley [Moo10] and Horridge [Hor11] claim that LBMs are not useful to find justifications (MinImps) for inconsistencies because the formula that represents inconsistencies in DLs, that is $T \sqsubseteq \perp$, has empty signature. However, Corollary 2.4.18 shows that the module for the empty signature actually preserves the MinImps for inconsistencies. Note that there are multiple ways to prove this, in particular using the inseparability relations and module properties studied by Sattler, Schneider and Zakharyaschev [SSZ09].

Corollary 2.4.18. Let $\mathcal{O}$ be an ontology in a $D L \mathfrak{L}$ and $x \in\left\{\top, \perp, \top \perp^{*}\right\}$ a module type, then $X \in x-\operatorname{LBM}(\mathcal{O}, \emptyset)$ for all $X \in \operatorname{MinImps}(\mathcal{O},\{\top \sqsubseteq \perp\}, \emptyset)$. 
Proof. Suppose that $\mathcal{O}$ is inconsistent, let $\Omega=\{\top \sqsubseteq \perp\}$ and $\Phi=\emptyset$. Then, $\operatorname{MinImps}(\mathcal{O}, \Omega, \Phi) \neq \emptyset$. Let $X \in \operatorname{MinImps}(\mathcal{O}, \Omega, \Phi)$. Since $X$ and $\mathcal{O}$ are inconsistent, for any $\alpha \in \mathfrak{L}$ we have: $X \models \alpha$ and $\mathcal{O}=\alpha$, regardless of $\alpha$ 's signature. Therefore, by justification-preservation $X$ must be part of all $x$-LBMs, in other words, $X \in x-\operatorname{LBM}(\mathcal{O}, \emptyset)$. Since $X$ is arbitrary, the same happens for every element of $\operatorname{MinImps}(\mathcal{O}, \Omega, \Phi)$, and thus $X \in x-\operatorname{LBM}(\mathcal{O}, \emptyset)$ for all $X \in \operatorname{MinImps}(\mathcal{O}, \Omega, \Phi)$.

Otherwise, if $\mathcal{O}$ is consistent, $\operatorname{MinImps}(\mathcal{O}, \Omega, \Phi)=\emptyset$ and the corollary is trivialised.

It is clear from monotonicity on signature enlargements (Proposition 2.4.8(3)) that the axioms in the module for the empty signature are a subset of every other module. These axioms are called global axioms [Del13]. On the other hand, there are also axioms that are not part of any module, they are a subset of the tautologies present in the ontology, called syntactic tautologies [Del13].

Example 2.4.19 explores this use-case to also illustrate how the LBMs are often more useful than simpler signature-based approaches, such as taking all the axioms connected to the input formula (strategy found both in Belief Change [Par99, CW15], as well as in Ontology Repair [HvHtT05, JQH09]).

Example 2.4.19. Consider the ontology MythEx:

$$
\begin{aligned}
\text { MythEx: }=\{ & \alpha_{1}: \text { Divinity } \sqcap \text { Human } \sqsubseteq \perp, \\
& \left.\alpha_{2}: \text { Human }(\text { hercules }), \alpha_{3}: \text { Divinity(hercules }\right), \\
& \left.\alpha_{4}: \text { Dog } \sqsubseteq \text { Animal }, \alpha_{5}: \text { Animal } \sqsubseteq \text { Organism }\right\}
\end{aligned}
$$

The connected component for the formula $\beta$ : Dog $\sqsubseteq$ Organism contains only $\alpha_{4}$ and $\alpha_{5}$. However, due to the inconsistency caused by the fact that Hercules is at the same time a human and a divinity, the connected fails to capture the whole $\operatorname{Min} \operatorname{Imps}(\operatorname{InCon},\{\beta\}, \emptyset)$. In the case of the locality-based modules, the set $\left\{\alpha_{1}, \alpha_{2}, \alpha_{3}\right\}$ is a subset of every LBM for $x \in\left\{\top, \perp, \top \perp^{*}\right\}$. Also, the LBM of MythEx for $\operatorname{sig}(\beta)$ is the whole ontology, larger than the connected component, but manages to capture the complete MinImps.

\subsubsection{Atomic Decomposition}

Del Vescovo [Del13] investigates which modularity methods are suitable to identify a structure in the ontologies concisely. Still, not even the locality-based modules were deemed adequate in this aspect. They fail because the naïve approach to structure ontology via LBMs requires computing an exponential number of locality-based modules [PS10].

The exponential number comes from the existence of redundant modules. These modules (henceforth called fake) can be composed as a union of other incomparable (w.r.t. set inclusion). A module is genuine if it is not fake.

The atomic decomposition, proposed by Del Vescovo et al. [DPSS11], provides a solution to this problem. It consists of a concise representation of every genuine module of an ontology that can be generated in polynomial time. While the atomic decomposition is defined for a more general class of modules, and not only the locality-based ones, for conciseness here onwards this thesis assumes that the $\mathrm{AD}$ is based on LBMs.

Within the atomic decomposition, the axioms are divided into partitions (atoms) according to co-occurrence in modules, that is if two axioms are present exactly in the same modules, then they belong to the same partition. Definition 2.4.20 describes this concept formally.

Definition 2.4.20 (Atom [Del13]). Let $\operatorname{AllMods}_{x}(\mathcal{O})$ be the set of all $x$-locality-based modules of an ontology $\mathcal{O}$ for a fixed module type $x$ in $\left\{\perp, \top, \top \perp^{*}\right\}$. The relation $\approx$ is the binary relation over $\mathcal{O}$ defined to hold between two axioms $\alpha, \beta \in \mathcal{O}$ if, for all $\mathcal{M} \in \operatorname{AllMods}_{x}(\mathcal{O}), \alpha \in \mathcal{M}$ iff $\beta \in \mathcal{M}$.

An $x$-atom $\mathfrak{a}$ of $\mathcal{O}$ is an equivalence class $[\alpha]_{\approx}$ for an axiom $\alpha \in \mathcal{O}$. Further, atoms ${ }_{x}(\mathcal{O})$ denotes the set of $x$-atoms of $\mathcal{O}$. 
Whenever the notion $x$ is irrelevant or clear from context the qualifier $x$ is omitted. In these situations, $x$-atoms are simply called atoms, the set of all LBMs of $\mathcal{O}$ is represented by $\operatorname{AllMods}(\mathcal{O})$, while atoms $(\mathcal{O})$ denotes the set of atoms of $\mathcal{O}$.

Definition 2.4.20 almost partitions the whole ontology: it misses the syntactic tautologies, the axioms that are never part of a module. There are two possible ways to circumvent this shortcoming. The first is to ignore them, as they have no logical impact (they are indeed tautologies), the second is to create an new partition $\mathfrak{t}$ that contains these axioms.

Additionally, the atoms can depend on one another. If an atom $\mathfrak{a}$ belongs to a module whenever $\mathfrak{b}$ also does, then $\mathfrak{a}$ depends on $\mathfrak{b}$. This concept is detailed in Definition 2.4.21.

Definition 2.4.21 (Dependency Between Atoms [Del13]). Let $\mathfrak{a}$ and $\mathfrak{b}$ be two atoms induced by $\approx$ over an ontology $\mathcal{O}$, for a fixed type of LBM $x \in\left\{\perp, \top, \top \perp^{*}\right\}$. An $x$-atom $\mathfrak{a}$ is $x$-dependent of an $x$-atom $\mathfrak{b}$ in $\mathcal{O}$ (in symbols, $\mathfrak{a} \succeq_{x}^{\mathcal{O}} \mathfrak{b}$ ) if, for every $x$-LBM $\mathcal{M} \in \operatorname{AllMods}_{x}(\mathcal{O})$ such that $\mathfrak{a} \subseteq \mathcal{M}$, it hold that $\mathfrak{b} \subseteq \mathcal{M}$.

As in Definition 2.4.20, the subscript indicating the LBM type is omitted whenever it is irrelevant or clear from context.

The strict part of the dependency relation forms a partially ordered set, which in turn determines the $x$-atomic decomposition of $\mathcal{O}$ (or simply $x-\operatorname{AD}(\mathcal{O})$ ) as the pair $\left(\operatorname{atoms}_{x}(\mathcal{O}), \succ_{x}^{\mathcal{O}}\right.$ ). As usual, we omit the specification of LBM type $x$ whenever doing so does not harm the interpretation of the text.

As illustration, consider the ontology AccountEx from Example 2.4.22, its atomic decomposition using $T \perp^{*}$-LBMs (i.e. the $T \perp^{*}$-AD of AccountEx) is shown in Figure 2.3. Each node represents an atom (in this case, each one has a single formula), and the edges represent the dependency relations.

Example 2.4.22 ([GW17]). Consider the ontology AccountEx:
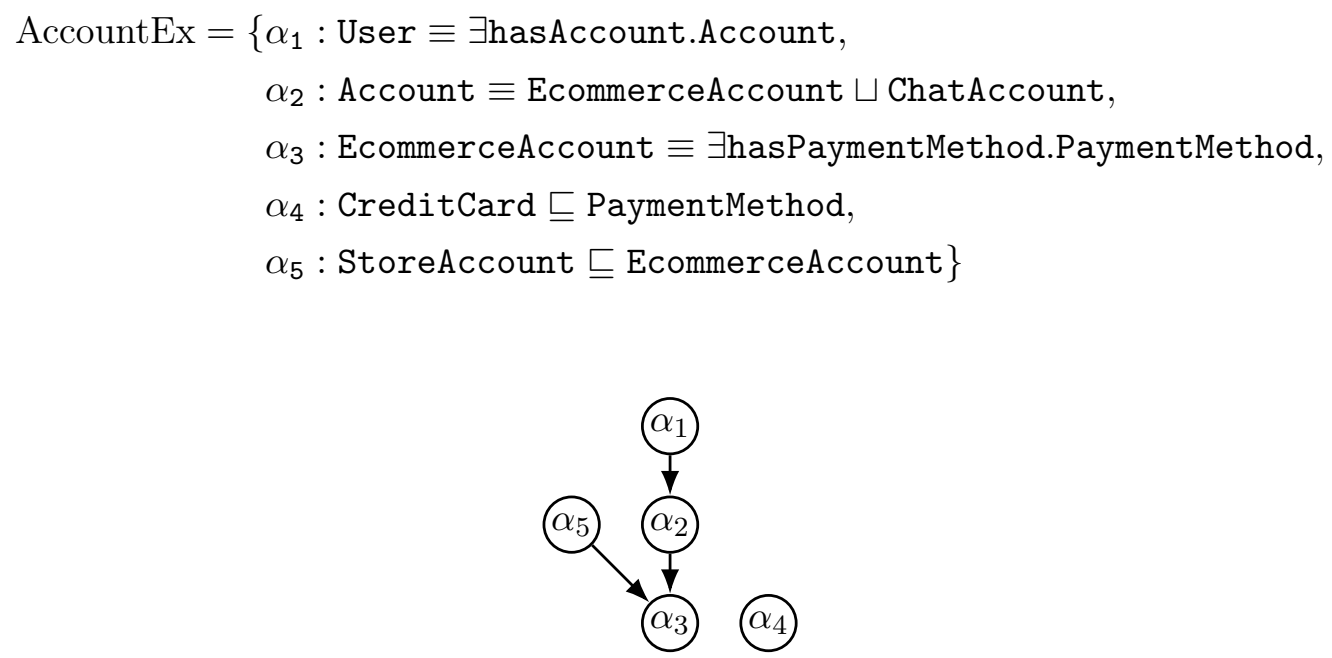

Figure 2.3: $T \perp^{*}-A D$ of the example ontology AccountEx

In the atomic decomposition, each atom represents a genuine module [DPSS11]. The genuine module for an atom contains the atom, and all other atoms that it depends on. Furthermore, the number of atoms is at most the number of axioms of the ontology, since no partition is empty. Besides, the AD can be computed in polynomial time [DPSS11, Tsa12], it requires at most one module extraction for each axiom in the ontology.

Although every module (genuine or fake) is represented in the AD, it is not easy to obtain the fake ones from the structure. The strategies for doing so using labels [Del13] and hypergraphs [MW14] suffer from having to calculate sets of exponential size.

Still, for the purposes of this thesis the AD may prove a convenient tool to mitigate the multiple outcome problem. In the $\mathrm{AD}$, it is easy to estimate which axioms participate in more or fewer 
modules. Moreover, the $\perp$ and T-ADs exhibit a strong relation with the class hierarchy [SQJH08, Del13, Mat16, GSW18], likely a reflection of Proposition 2.4.23.

Proposition 2.4.23 ([Del13, Mat16]). Let $\mathcal{O}$ be an ontology and $\Sigma$ a signature, Then:

1. $\mathcal{M}=\perp-\operatorname{LBM}(\mathcal{O}, \Sigma)$ is subsumer-complete, that is for all concept names $\mathrm{A} \in \Sigma \cup \operatorname{sig}(\mathcal{M})$ and $\mathrm{B} \in \operatorname{sig}(\mathcal{O}): \mathcal{M} \models \mathrm{A} \sqsubseteq \mathrm{B}$ iff $\mathcal{O} \models \mathrm{A} \sqsubseteq \mathrm{B}$.

2. $\mathcal{M}=\top-\operatorname{LBM}(\mathcal{O}, \Sigma)$ is subsumee-complete, that is for all concept names $\mathrm{B} \in \Sigma \cup \operatorname{sig}(\mathcal{M})$ and $\mathrm{A} \in \operatorname{sig}(\mathcal{O}): \mathcal{M} \models \mathrm{A} \sqsubseteq \mathrm{B}$ iff $\mathcal{O} \models \mathrm{A} \sqsubseteq \mathrm{B}$.

The exploration of the $\mathrm{AD}$ as a mean to alleviate the multiple outcome problem is deferred to Chapter 7, including a more comprehensive discussion on the correlation of the AD with the class hierarchy. 


\section{Chapter 3}

\section{Modularity in Ontology Repair}

This chapter discusses the state-of-art concerning the usage of modules in Ontology Repair, highlights the margins for improvements in this area and the gaps in the literature concerning the impact of such techniques. Furthermore, this chapter gathers arguments for and against the usage of modules in Belief Change and Ontology Repair. Ultimately, the discussion guides the research questions and hypotheses, which shape our theoretical and empirical pursuits that follow in the next chapters.

\subsection{State-of-art}

Currently, justification-preserving modules are already in use for Ontology Debug and Repair, especially in MinImp-based approaches [SQJH08, BS08, Moo10, Nor11, Hor11]. These approaches show that modularity is indeed beneficial concerning computational resources consumed. The most straightforward and common approach consists in extracting a JPM for the desired input before computing the actual MinImps [SQJH08, BS08, Hor11].

A finer-grained approach is explored by Moodley [Moo10] that proposes the extraction of the module at the enlargement phase of the black-box computation of a MinImp (as in Algorithm 4). Nortje [Nor11] instead employs modularity in a glass-box solution. The technique devised relies on a particular modularity approach and aims to MinImps in logics from the $\mathcal{E} \mathcal{L}$ family.

Concomitantly to optimisation, there are also modularity-inspired approaches to solve the problem of multiple outcomes. One example is the variant of Algorithm 1 designed by Ji, Qi and Haase [JQH09] which can prioritise the generation of the most important MinImps according to a criterion inspired on Baader and Suntisrivaraporn's reachability-based modules [BS08].

There is little to no information on the effects of modularity on MaxNons-based approaches both in Belief Change and Ontology Repair areas. This scarcity is not surprising if given that MinImps are much more prevalent in Ontology Repair than MaxNons. The significant difference in the number of publications with MinImp-based techniques $\left[\mathrm{BCM}^{+} 03, \mathrm{SC} 03, \mathrm{Sch} 05, \mathrm{BPS} 07\right.$, SQJH08, BS08, JQH09, Hor11, Nor11] versus of those that use MaxNons-based ones [BP10, RRW14, CW15, IMMP17] is evidence of such disparity.

\subsection{Advantages and Disadvantages}

As mentioned in Section 2.4, modules are being used to improve the computational performance of algorithms that rely on reasoning many applications. However, few studies quantify the impact of modularisation in performance. Furthermore, some empirical studies in this sense do not compare the computation of MinImps with and without modularity [BS08], or only evaluate the reduction in the number of axioms, but do not assess the difference in computational resources consumed [Moo10, Nor11]. A notable exception is due to Suntisrivaraporn et al. [SQJH08] where they compare the 
computation of MinImps in large $\mathcal{E} \mathcal{L}$ ontologies (namely, NCI-thesaurus ${ }^{1}$, Galen ${ }^{2}$ and $\mathrm{GO}^{3}$ ) is improved by orders of magnitude using $\perp$-locality modules.

Subsections 3.2.1 and 3.2.2 support the decision of using modules to improve Ontology Repair algorithms and, at the same time, highlight the need for further studies to quantify their actual influence on performance and potential interference with choice mechanisms in Belief Change.

\subsubsection{Arguments in Favour}

Aside from the gains in execution time attested by a few studies in the literature [SQJH08], modules almost always reduce the search space of reasoning algorithms [BS08, Moo10, Hor11]. Tractable approaches to modularity, such as LBMs, end up including more axioms than needed. Even so, such techniques are effective in removing axioms that can be safely ignored and downsizing ontologies to more manageable sizes $\left[\mathrm{DKP}^{+} 12\right]$.

For Ontology Repair, one of the main potential benefits of reducing the number of axioms concerns the way how each MinImp and MaxNon is computed in black-box methods. In black-box implementations based on enlargement and shrinking heuristics (as those introduced in Subsection 2.3.4) smaller ontologies generally incur in fewer reasoner calls per element computed.

Furthermore, Matentzoglu [Mat16] carried out a careful evaluation of the effects of $\perp$-localitybased modules in Ontology Classification. The study shows that, either due to test avoidance (the possibility of doing fewer operations during reasoning) or "easyfication" (turning tests easier to compute by a reasoner), modules reduce time spent during reasoning.

As for the atomic decomposition, many of its benefits, such as tractability, stem from LBMs. As discussed in Subsection 2.4.4, the main benefit of the AD is the ability to represent, in a polynomial way, a structure between all the fundamental (called genuine) modules of an ontology. Further, as with LBMs, there is a well-tested implementation available via the OWL API . Above all, it is the best approach for ontology structuring, according to Del Vescovo [Del13].

Another fact that is given less attention is that $\perp$ and $T$-ADs can establish sequences of interpretations of the terms of the ontology, forming a chain of conservative extensions. Moreover, both types of $\mathrm{AD}$ are closely related to the class hierarchy of an ontology. Chapter 7 investigates this relation, extending previous studies [GSW18].

\subsubsection{Arguments Against}

One of the most persuasive arguments against modularity is the overhead (i.e. the additional resources required) in extracting them [Mat16]. Even polynomial approaches can be deemed expensive for large ontologies. The overhead has to be taken more into account when having modules need to be extracted more than one time during the whole process. However, as the popularity of modules may already hint, the overhead is often compensated by the gains in running time in most cases.

Another argument is that, in a few situations, modules can actually make reasoning more difficult. However, the study carried by Matentzoglu shows that this only occurs in a very small portion of the cases [Mat16] (0.34\% in one approach of sampling, $0.43 \%$ in the other).

Another argument is that, in rare situations, modules can make reasoning more difficult [Mat16]. However, the study carried by Matentzoglu shows that this only occurs in a tiny portion of the cases [Mat16](0.34\% in one approach of sampling, $0.43 \%$ in the other). Still, for Ontology Repair tasks the figures might differ.

The main issue regarding the atomic decomposition is that it may require one module extraction for each axiom in the ontology. This process is polynomial for LBMs, but computationally intensive nonetheless.

\footnotetext{
${ }^{1}$ https://ncithesaurus.nci.nih.gov/

${ }^{2}$ http://geneontology.org/

${ }^{3}$ http://geneontology.org/

${ }^{3}$ https://owlcs.github.io/owlapi/
} 
In Chapter 7, the AD is used as a basis for stratification methods. While Guimarães and Wassermann [GW17] proposed the use of the $T \perp^{*}$-AD for similar purposes, only $T$-AD and $\perp$-AD have meaning relations with the class hierarchy and chains of conservative extensions [Del13]. Also, there is no simple way to update the structure after a modification in the ontology, incurring in further costs to compute it for the resulting ontology if needed [KDS12].

There are also other general problems of the AD that hinder its usage, such as the lack of an efficient way to build arbitrary modules from it. The approaches that address this problem usually depend on labels that can be exponentially large on the length of an axiom, or on hypergraphs transformations which also display the same exponential issue in the general case [MW14].

Another argument against both modules and the AD is that many ontologies end up having an atom that includes the vast majority of the axioms in the ontology. In these cases, the AD is almost useless for distinguishing axioms and the structure also indicates that the modules of the ontology are prone to be large (since atoms do not split across modules) [Del13].

\subsection{Research Agenda}

Among the shortcomings of existing approaches Ontology Repair and Belief Change for DL bases are the high computational costs associated to constructing MinImps and MaxNons, and the exponential size of such sets [BPS07, Peñ09, Cób14]. The first will be referred as the computational problem, the latter as the choice problem.

Based on the state-of-art and arguments presented in this chapter, there are various open questions regarding the use of modules in Ontology Repair and Belief Change to address the issued mentioned earlier. This study focuses on the following three research questions:

Q1 How to integrate modules with the theory of Belief Base Change for Description Logics (discussed in Section 2.3)?

Q2 How significant is the impact of $T \perp^{*}$-LBMs on the efficiency of the black-box computation of MinImps and MaxNons (with the algorithms shown in Subsection 2.3.4)? Specifically, concerning running time, maximum computational memory consumed, and the number of entailment queries to the reasoner.

Q3 Does the modular structure, via atomic decomposition, provides a meaningful and effective way to mitigate the multiple outcome issue?

The first question involves the identification of sufficient properties that modules must have to preserve the postulates in Belief Change. If possible, the postulates should remain unchanged, as they provide a solid basis to describe rational change. This thesis address this question giving special attention to justification-preserving modules, introduced by Definition 2.4.2.

More specially, Chapter 4 investigates whether JPMs also preserve MinImps and MaxNons. Also, it analyses effects on incision functions and selection functions. Finally, it uses preliminary results given in Chapter 2 to define algorithms that update a module while the sets are being computed, providing a better approximation.

Meanwhile, Chapters 5 and 6 are devoted to the second research question (Q2). This question also relates to the arguments concerning the overhead of modularity. If the impact proves to be harmful in a significant amount of cases, then future studies need to be more careful when using modularity. On the other hand, if modules prove to be advantageous, more evidence will be added for their use when computing MinImps, and a solid argument for using them when computing MaxNons. Note that it is even possible that the impact on the calculus of MinImps and MaxNons differ.

It is also important to note that, in terms of methodology, the experiments carried out in this thesis extend the ones published by Suntisrivaraporn et al. [SQJH08]. Here, the experiment involves more ontologies and evaluates two approaches for modularised calculus of MinImps based 
on Algorithm 1 (these approaches will be specified in Chapter 4). Further, it also includes evaluation for MaxNons-based methods.

Chapter 5 presents the experimental framework devised to answer (Q2) and related minor questions, such as the ones we mentioned previously. It describes not only the implementation but also the most important decisions that had to be made during its design. Posteriorly, Chapter 6 details the experiments constructed to assess the impact of modularity. Also, it discusses the results obtained and the conclusions drawn from the data gathered.

Next, Chapter 7 that address the third research question (Q3). Its primary approach consists of extending previous studies in this direction [GSW18]. Still, it has novel experiments, results and discussions. In summary it proposed stratification methods and compares then with existing ones in a general setting and in the particular case of Ontology Repair. Further, correlation between methods is measured using statistical tools.

This thesis does not aim to give a definitive answer with respect to the use of modules in Ontology Repair and Belief Change implementations, but to fill the gaps in knowledge so that researchers and engineers have more material to decide on the use of modules in these areas. 


\section{Chapter 4}

\section{Modularity-Aware Belief Base Change in Description Logics}

This chapter concentrates most of the theoretical results concerning modules, in particular, the justification-preserving ones of Definition 2.4.2 and the constructions and operations introduced in Section 2.3. Further, it also presents a method to compute MinImps that depends on specific properties of the locality-based modules.

Whenever said otherwise the modules considered in this chapter (e.g. denoted as $\bmod (\mathcal{O}, A)$ ) will always be JPMs as in Definition 2.4.2.

\subsection{Justification-Preservation in Belief Change of Description Log- ics Bases}

As mentioned in Subsection 2.4.2, there are already modularity-based approaches to compute $\operatorname{Min} \operatorname{Imps}(\mathcal{O}, \Omega, \emptyset)$, which corresponds to the kernel set or the set of all justifications (among other names as discussed in Subsection 2.3.3). These proposals rely on modules that are justificationpreserving as in Definition 2.4.2.

In what follows, especially when dealing with MaxNons, it is necessary to consider that $\bmod (\mathcal{O} \cup$ $\Phi, \Omega) \subseteq \mathcal{O}$ does not always hold. In this sense, it is more convenient to consider the intersection of the module with the ontology, instead of the module alone. For clarity, more notation is introduced: $\bmod (\mathcal{O}, \Omega, \Phi)=\bmod (\mathcal{O} \cup \Phi, \Omega) \cap \mathcal{O}$ Moreover, $\bmod (\mathcal{O}, \Omega, \Phi)$ denotes a module for a setting $(\mathfrak{L}, \mathcal{O}, \Omega, \Phi)$.

Proposition 4.1.1 (MinImp-preservation). Let $(\mathfrak{L}, \mathcal{O}, \Omega, \Phi)$ be a finite DL-setting. Then:

$$
\operatorname{MinImps}(\mathcal{O}, \Omega, \Phi)=\operatorname{MinImps}(\bmod (\mathcal{O}, \Omega, \Phi), \Omega, \Phi)
$$

Proof. Let $\mathcal{M}=\bmod (\mathcal{O}, \Omega, \Phi)$. One side of the proof is an immediate consequence of monotonicity and justification-preservation: if $X \in \operatorname{MinImps}(\mathcal{M}, \Omega, \Phi)$ then, $X \subseteq \mathcal{O}, \operatorname{Cn}(X \cup \Phi) \cap \Omega \neq \emptyset$ and there is no $X^{\prime} \subset X \subseteq \mathcal{M} \subseteq \mathcal{O}$ that is also a minimal $\Omega$-implying subset of $\mathcal{O}$ w.r.t. $\Phi$. Hence, $X \in \operatorname{MinImps}(\mathcal{O}, \Omega, \Phi)$.

Now, for the other direction, let $X \in \operatorname{MinImps}(\mathcal{O}, \Omega, \Phi)$. Then there is a $Y \subseteq X \cup \Phi$ with $Y \in \operatorname{MinImps}(\mathcal{O} \cup \Phi, \Omega)$. Since $\bmod (\mathcal{O} \cup \Phi, \Omega)$ is a JPM, $Y \subseteq \mathcal{M}$. In this way, $X \subseteq \mathcal{M}$ and $\operatorname{Cn}(X \cup \Phi) \cap \Omega \neq \emptyset$. Moreover, there can be no $X^{\prime} \subset X \subseteq \mathcal{M}$ that is minimal $\Omega$-implying w.r.t. $\Phi$, therefore $X \in \operatorname{MinImps}(\mathcal{M}, \Omega, \Phi)$.

In a certain sense, Proposition 4.1.1 proves that justification-preservation entails MinImppreservating (with a few adjustments). As for MaxNons, Lemma 4.1.2 gives the first result on what happens with this construction upon modularisation.

Lemma 4.1.2. Let $(\mathfrak{L}, \mathcal{O}, \Omega, \Phi)$ be a setting and $\mathcal{M}=\bmod (\mathcal{O}, \Omega, \Phi)$. Then $X \in \operatorname{MaxNons}(\mathcal{O}, \Omega, \Phi)$ iff $X \cap \mathcal{M} \in \operatorname{MaxNons}(\mathcal{M}, \Omega, \Phi)$. 
Proof. The first part of the demonstration shows that if $X \in \operatorname{MaxNons}(\mathcal{O}, \Omega, \Phi)$, then $X \cap \mathcal{M} \in$ $\operatorname{MaxNons}(\mathcal{M}, \Omega, \Phi)$. Let $X$ be any such set:

1. $X \cap \mathcal{M} \subseteq \mathcal{M}$.

2. By monotonicity, and since $\operatorname{Cn}(X \cup \Phi) \cap \Omega=\emptyset$, we get $\operatorname{Cn}((X \cap \mathcal{M}) \cup \Phi) \cap \Omega=\emptyset$.

3. Suppose there is a set $Y$ with $X \cap \mathcal{M} \subset Y \subseteq \mathcal{M}$ and such that $\operatorname{Cn}(Y \cup \Phi) \cap \Omega=\emptyset$. By monotonicity, there is a $Z \in \operatorname{MaxNons}(\mathcal{O}, \Omega, \Phi)$ with $Y \subseteq Z$. Therefore $X \cap \mathcal{M} \subset Y \subseteq Z \cap \mathcal{M}$, from which we derive $X \cap \mathcal{M} \subset Z \cap \mathcal{M}$, and thus $X \subset Z$. This strict set inclusion contradicts our assumption that $X \in \operatorname{MaxNons}(\mathcal{O}, \Omega, \Phi)$ (thus, maximal) showing that such $Y$ cannot exist.

Therefore, $X \cap \mathcal{M} \in \operatorname{MaxNons}(\mathcal{M}, \Omega, \Phi)$.

For the other direction, the demonstration shows that if $Y \in \operatorname{MaxNons}(\mathcal{M}, \Omega, \Phi)$ then $X_{Y}=$ $(\mathcal{O} \backslash \mathcal{M}) \cup Y \in \operatorname{MaxNons}(\mathcal{O}, \Omega, \Phi)$ :

1. $X_{Y} \subseteq \mathcal{O}$.

2. Assume that $\operatorname{Cn}\left(X_{Y} \cup \Phi\right) \neq \emptyset$. Then, let $Z \subseteq\left(X_{Y} \cup Y\right)$ with $Z \in \operatorname{MinImps}\left(X_{Y}, \Omega, \Phi\right)$ (such $Z$ exists due to compactness). It is also known that $Z \subseteq Y$ because $\mathcal{M}$ is a justificationpreserving module. Using monotonicity, one can derive that $\operatorname{Cn}(Y \cup \Phi) \cap \Omega \neq \emptyset$, violating our assumption that $Y \in \operatorname{MaxNons}(\mathcal{M}, \Omega, \Phi)$. In this way, there can be no such $Z$ and thus $\operatorname{Cn}\left(X_{Y} \cup \Phi\right)=\emptyset$.

3. Suppose there is a set $Z \in \operatorname{MaxNons}(\mathcal{O}, \Omega, \Phi)$ with $X_{Y} \subset Z$. This implies that $\operatorname{Cn}((Z \cap \mathcal{M}) \cap$ $\Omega)=\emptyset$ because of monotonicity. Also, it is known that $Z \cap \mathcal{M} \subseteq \mathcal{M}$. These properties of $Z \cap \mathcal{M}$ contradict the hypothesis that $Y \in \operatorname{MaxNons}(\mathcal{M}, \Omega, \Phi)$. In other words, there can be no strict superset of $X_{Y}$ in $\operatorname{MaxNons}(\mathcal{O}, \Omega, \Phi)$.

Observe that the items proved that $X_{Y}=(\mathcal{O} \backslash \mathcal{M}) \cup Y \in \operatorname{MaxNons}(\mathcal{O}, \Omega, \Phi)$. Also, since $Y \subseteq \mathcal{M}: X_{Y} \cap \mathcal{M}=((\mathcal{O} \backslash \mathcal{M}) \cup Y) \cap \mathcal{M}=Y$. Thus, if $X \cap \mathcal{M} \in \operatorname{MaxNons}(\mathcal{M}, \Omega, \Phi)$ then $X \in \operatorname{MaxNons}(\mathcal{O}, \Omega, \Phi)$.

As both directions are valid, the proof is concluded.

Lemma 4.1.2 makes statements only about individual MaxNons, Proposition 4.1.3 shows that there is a similar result for the whole set.

Proposition 4.1.3. Let $(\mathfrak{L}, \mathcal{O}, \Omega, \Phi)$ be a finite $D L$-setting and $\mathcal{M}=\bmod (\mathcal{O}, \Omega, \Phi)$, then

$$
\operatorname{MaxNons}(\mathcal{O}, \Omega, \Phi)=\{X \cup \mathcal{O} \backslash \mathcal{M} \mid X \in \operatorname{MaxNons}(\mathcal{M}, \Omega, \Phi)\}
$$

Proof. Immediate consequence from Lemma 4.1.2 and the equality: $X \cap \mathcal{M}=X \backslash(\mathcal{O} \backslash \mathcal{M})$ for any $X \subseteq \mathcal{O}$.

The results demonstrated, in particular Propositions 4.1.1 and 4.1.3, show that it is possible to compute all the belief base change operations discussed in Subsection 2.3.3 by constructing MinImps and MaxNons using JPMs.

However, besides computing these sets, contraction and revision functions also choose axioms from the MinImps or a subset of the MaxNons. The remaining of this section investigates how justification-preserving modules affect this selection. The focus will be on revision as it has been discussed in Subsection 2.3.3 that contraction operations correspond to the particular case where $\Phi=\emptyset$.

For MinImps-based approaches (using kernel sets, or justifications), the result is straightforward as Corollary 4.1.4 shows. 
Corollary 4.1.4. Let $(\mathfrak{L}, \mathcal{O}, \Omega, \Phi)$ be a finite DL-setting, $\mathcal{M}=\bmod (\mathcal{O}, \Omega, \Phi)$, and $\operatorname{ikr}_{\mathbf{f}_{1}}$ and $\operatorname{ekr}_{\mathbf{f}_{2}}$ be, respectively internal and external kernel revision operations as in Definitions 2.3.13 and 2.3.19. Then:

$$
\begin{aligned}
& \text { 1. } \operatorname{ikr}_{\mathbf{f}_{1}}(\mathcal{O}, \Omega, \Phi)=\Phi \cup\left(\mathcal{O} \backslash \mathbf{f}_{1}(\operatorname{MinImps}(\mathcal{M}, \Omega, \Phi), \Omega, \Phi)\right) \text {. } \\
& \text { 2. } \operatorname{ekr}_{\mathbf{f}_{2}}(\mathcal{O}, \Omega, \Phi)=(\mathcal{O} \cup \Phi) \backslash \mathbf{f}_{2}(\operatorname{MinImps}(\mathcal{M}, \Omega, \emptyset), \Omega, \Phi) \text {. }
\end{aligned}
$$

Proof. As Proposition 4.1.1 guarantees that replacing the ontology by a module does not change the MinImps, then:

$$
\mathbf{f}(\operatorname{MinImps}(\mathcal{O}, \Omega, \Phi), \Omega, \Phi)=\mathbf{f}(\operatorname{MinImps}(\mathcal{M}, \Omega, \Phi), \Omega, \Phi)
$$

and

$$
\mathbf{f}(\operatorname{MinImps}(\mathcal{O}, \Omega, \emptyset), \Omega, \Phi)=\mathbf{f}(\operatorname{MinImps}(\mathcal{M}, \Omega, \emptyset), \Omega, \Phi)
$$

for any incision function $\mathbf{f}$. This holds for any of three types of incision functions (from Definitions 2.3.6, 2.3.17 and 2.3.18) because the only characteristics required are the domain definition, which is the same for all three, and the fact that they are functions. In this way, the equalities in the corollary are valid.

Corollary 4.1.5 shows an analagous result for MaxNons.

Corollary 4.1.5. Let $(\mathfrak{L}, \mathcal{O}, \Omega, \Phi)$ be a finite DL-setting, $\mathcal{M}=\bmod (\mathcal{O}, \Omega, \Phi)$, and ipmr $_{\mathbf{g}_{1}}$ and epmr $_{\mathbf{g}_{2}}$ be, respectively external and internal partial meet revision operations as in Definitions 2.3.15 and 2.3.23.

$$
\begin{aligned}
& \text { 1. } \left.\operatorname{ipmr}_{\mathbf{g}_{1}}(\mathcal{O}, \Omega, \Phi)=\Phi \cup \bigcap \mathbf{g}_{1}(\{X \cup(\mathcal{O} \backslash \mathcal{M})) \mid X \in \operatorname{MaxNons}(\mathcal{M}, \Omega, \Phi)\}, \Omega, \Phi\right) . \\
& \text { 2. } \operatorname{epmr}_{\mathbf{g}_{2}}(\mathcal{O}, \Omega, \Phi)=\bigcap \mathbf{g}_{2}(\{X \cup((\mathcal{O} \cup \Phi) \backslash \mathcal{M}) \mid X \in \operatorname{MaxNons}(\mathcal{M}, \Omega, \emptyset)\}, \Omega, \Phi) .
\end{aligned}
$$

Proof. Analogous to Corollary 4.1.4's proof, using Proposition 4.1.3 and the fact that $\mathbf{g}_{1}$ and $\mathbf{g}_{2}$ are selection functions.

Note that Corollary 4.1.4 allows us to not only use modules to compute MinImps but also to apply the same incision function over the modularised and classical results (since they are the same). Although kernel operations are more general than partial meet ones, Corollary 4.1.5 does not warrant the same property for selection functions.

Proposition 4.1.3 shows that, as opposed to MinImps, the MaxNons of the module can be different from the MaxNons of the whole ontology. Even if we can build the later using the former, the domain of the selection functions is likely to change since the MaxNons differ in general.

A closer look must be taken at the effect of modularisation on selection functions to find a relation for partial meet constructions that resembles the one for kernel constructions. In this direction, Proposition 4.1.3 will be strengthened with a bijection between MaxNons in the ontology and the ones in its module. Proposition 4.1.6 is an intermediate step to prove the existence of this function.

Proposition 4.1.6. Let $\mathcal{M}=\bmod (\mathcal{O}, \Omega, \Phi)$ be a JPM for DL-setting $(\mathfrak{L}, \mathcal{O}, \Omega, \Phi)$. Then: $\mathcal{O} \backslash \mathcal{M} \subseteq$ $\bigcap \operatorname{MaxNons}(\mathcal{O}, \Omega, \Phi)$.

Proof. Clearly $\mathcal{O} \backslash \mathcal{M} \subseteq \mathcal{O}$. Additionally, Proposition 4.1.1 implies that $\operatorname{Cn}(\mathcal{O} \backslash \mathcal{M} \cup \Phi) \cap \Omega=\emptyset$. Due to monotonicity, $\mathcal{O} \backslash \mathcal{M} \subseteq X$, for any $X \in \operatorname{MaxNons}(\mathcal{O}, \Omega, \Phi)$.

In the proof of Proposition 4.1.7, Proposition 4.1.6's role is to show that the mapping Proposition 4.1.3's statement already defines a bijection between the MaxNons in the module and those in the ontology. 
Proposition 4.1.7. Let $\bmod (\mathcal{O}, \Omega, \Phi)$ be a JPM function for a finite $D L$-setting $(\mathfrak{L}, \mathcal{O}, \Omega, \Phi)$. Then, the relation $m(X)=X \backslash(\mathcal{O} \backslash \mathcal{M})$, or equivalently, $m(X)=X \cap \mathcal{M}$ is a bijective function from $\operatorname{MaxNons}(\mathcal{O}, \Omega, \Phi)$ to $\operatorname{MaxNons}(\mathcal{M}, \Omega, \Phi)$.

Proof. It is evident that if $X=X^{\prime} \in \operatorname{MaxNons}(\mathcal{O}, \Omega, \Phi), m(X)=m\left(X^{\prime}\right)$, so $m$ is a function. If $X, X^{\prime} \in \operatorname{MaxNons}(\mathcal{O}, \Omega, \Phi)$ and $m(X)=m\left(X^{\prime}\right)$ we have:

$$
\begin{aligned}
X & =(X \cap \mathcal{M}) \amalg(X \cap(\mathcal{O} \backslash \mathcal{M})) \\
& =(X \cap \mathcal{M}) \amalg(\mathcal{O} \backslash \mathcal{M}) \\
& =\left(X^{\prime} \cap \mathcal{M}\right) \amalg(\mathcal{O} \backslash \mathcal{M}) \\
& =\left(X^{\prime} \cap \mathcal{M}\right) \amalg\left(X^{\prime} \cap(\mathcal{O} \backslash \mathcal{M})\right) \\
& =X^{\prime}
\end{aligned}
$$

(Proposition 4.1.6)

Therefore, $m$ is injective.

The function $m$ is also surjective because Lemma 4.1.2 ensures that if $Y \in \operatorname{MaxNons}(\mathcal{M}, \Omega, \Phi)$, then there is an $X \in \operatorname{MaxNons}(\mathcal{O}, \Omega, \Phi)$ such that $Y=m(X)$. Hence, $m$ is a bijective function as stated.

The question now is to determine when a selection function $\mathbf{g}$ over MaxNons and a selection function $\mathbf{g}^{\prime}$ over $\operatorname{MaxNons}(\mathcal{M}, \Omega, \Phi)$ are such that: $X \in \mathbf{g}(\operatorname{MaxNons}(\mathcal{O}, \Omega, \Phi), \Omega, \Phi)$ iff $X \cap \mathcal{M} \in$ $\mathbf{g}^{\prime}(\operatorname{MaxNons}(\mathcal{M}, \Omega, \Phi), \Omega, \Phi)$. Functions $\mathbf{g}$ and $\mathbf{g}^{\prime}$ satisfying these conditions will be called isomorphic w.r.t. $\mathcal{M}$. The importance of having isomorphic selection functions as such becomes evident when comparing Corollary 4.1.5 and Corollary 4.1.8.

Corollary 4.1.8. Let $\mathcal{M}=\bmod (\mathcal{O}, \Omega, \Phi)$ be a JPM for a setting $(\mathfrak{L}, \mathcal{O}, \Omega, \Phi)$, and let $\mathrm{ipmr}_{\mathbf{g}_{1}}$ and epmr $_{\mathbf{g}_{2}}$ be, respectively internal and external kernel revision operation as in Definitions 2.3.15 and 2.3.23, Then if $\mathbf{g}_{1}^{\prime}$ and $\mathbf{g}_{2}^{\prime}$ are isomorphic to $\mathbf{g}_{1}$ and $\mathbf{g}_{2}$ w.r.t. $\mathcal{M}$, respectively, then:

1. $\operatorname{ipmr}_{\mathbf{g}_{1}}(\mathcal{O}, \Omega, \Phi)=(\mathcal{O} \backslash \mathcal{M}) \cup \Phi \cup \bigcap \mathbf{g}_{1}^{\prime}(\operatorname{MaxNons}(\mathcal{M}, \Omega, \Phi), \Omega, \Phi)$.

2. $\operatorname{epmr}_{\mathbf{g}_{2}}(\mathcal{O}, \Omega, \Phi)=(\mathcal{O} \backslash \mathcal{M}) \cup \bigcap \mathbf{g}_{2}^{\prime}(\operatorname{MaxNons}(\mathcal{M}, \Omega, \emptyset), \Omega, \Phi)$.

Proof.

$$
\begin{array}{rll}
\operatorname{ipmr}_{\mathbf{g}_{1}}(\mathcal{O}, \Omega, \Phi) & =\Phi \cup \bigcap \mathbf{g}_{1}(\operatorname{MaxNons}(\mathcal{O}, \Omega, \Phi), \Omega, \Phi) & \\
= & \Phi \cup \bigcap\left\{X \cup(\mathcal{O} \backslash \mathcal{M}) \mid X \in \mathbf{g}_{1}^{\prime}(\operatorname{MaxNons}(\mathcal{M}, \Omega, \Phi), \Omega, \Phi)\right\} & \text { (isomorphism) } \\
= & \Phi \cup \bigcap(\mathcal{O} \backslash \mathcal{M}) \cup \mathbf{g}_{1}^{\prime}(\operatorname{MaxNons}(\mathcal{M}, \Omega, \Phi), \Omega, \Phi) \\
= & (\mathcal{O} \backslash \mathcal{M}) \cup \Phi \cup \bigcap \mathbf{g}_{1}^{\prime}(\operatorname{MaxNons}(\mathcal{M}, \Omega, \Phi), \Omega, \Phi) & \\
\operatorname{epmr}_{\mathbf{g}_{2}}(\mathcal{O}, \Omega, \Phi) & =\bigcap \mathbf{g}_{2}(\operatorname{MaxNons}(\mathcal{O} \cup \Phi, \Omega, \emptyset), \Omega, \Phi) \\
& =\bigcap\left\{X \cup(\mathcal{O} \backslash \mathcal{M}) \mid X \in \mathbf{g}_{2}^{\prime}(\operatorname{MaxNons}(\mathcal{M}, \Omega, \emptyset), \Omega, \Phi)\right\} \quad \text { (isomorphism) } \\
& =\bigcap(\mathcal{O} \backslash \mathcal{M}) \cup \mathbf{g}_{2}^{\prime}(\operatorname{MaxNons}(\mathcal{M}, \Omega, \emptyset), \Omega, \Phi) \\
& =(\mathcal{O} \backslash \mathcal{M}) \cup \bigcap \mathbf{g}_{2}^{\prime}(\operatorname{MaxNons}(\mathcal{M}, \Omega, \emptyset), \Omega, \Phi) &
\end{array}
$$

Next, Proposition 4.1.9 shows that a selection function always has an isomorphic counterpart w.r.t a JPM (for the same setting). 
Proposition 4.1.9. Let $\mathcal{M}$ and $\mathcal{M}^{\prime}$ be justification-preserving modules for a finite DL-setting $(\mathfrak{L}, \mathcal{O}, \Omega, \Phi)$. If $\operatorname{MaxNons}(\mathcal{M}, \Omega, \Phi)=\operatorname{MaxNons}\left(\mathcal{M}^{\prime}, \Omega, \Phi\right)$ then, $\mathcal{M}=\mathcal{M}^{\prime}$.

Proof. From Lemma 4.1.2 $X \in \operatorname{MaxNons}(\mathcal{M}, \Omega, \Phi)$ iff $X \cup(\mathcal{O} \backslash \mathcal{M}) \in \operatorname{MaxNons}(\mathcal{O}, \Omega$, $\Phi)$. Since $\operatorname{MaxNons}(\mathcal{M}, \Omega, \Phi)=\operatorname{MaxNons}\left(\mathcal{M}^{\prime}, \Omega, \Phi\right)$ :

$$
\begin{aligned}
X \cup(\mathcal{O} \backslash \mathcal{M}) & =X \cup\left(\mathcal{O} \backslash \mathcal{M}^{\prime}\right) & & \\
X \amalg(\mathcal{O} \backslash \mathcal{M}) & =X \amalg\left(\mathcal{O} \backslash \mathcal{M}^{\prime}\right) & & \left(X \subseteq \mathcal{M} \cap \mathcal{M}^{\prime}\right) \\
(\mathcal{O} \backslash \mathcal{M}) & =\left(\mathcal{O} \backslash \mathcal{M}^{\prime}\right) & & \\
\mathcal{M} & =\mathcal{M}^{\prime} & & \left(\mathcal{M} \cup \mathcal{M}^{\prime} \subseteq \mathcal{O}\right)
\end{aligned}
$$

For any selection function $\mathbf{g}$ and setting $(\mathfrak{L}, \mathcal{O}, \Omega, \Phi)$, one can produce an isomorphic $\mathbf{g}^{\prime}$ w.r.t. $\bmod (\mathcal{O}, \Omega, \Phi)$ by defining it as function of $\mathbf{g}$ :

$$
\begin{aligned}
\mathbf{g}_{\mathbf{g}}^{\prime}(\operatorname{MaxNons}(\mathcal{M}, \Omega, \Phi), \Omega, \Phi)=\{ & X \in \operatorname{MaxNons}(\mathcal{M}, \Omega, \Phi) \\
& \mid X \cup(\mathcal{O} \backslash \mathcal{M}) \in \mathbf{g}(\operatorname{MaxNons}(\mathcal{O}, \Omega, \Phi), \Omega, \Phi)\}
\end{aligned}
$$

Note that Proposition 4.1.9 ensures that $\mathbf{g}_{\mathbf{g}}^{\prime}$ from Equation (4.1) is indeed a function because it does not depend on $\mathcal{M}$ at all. However, with this simple method, a selection function still evaluates the MaxNons of the original ontology. In an ideal scenario, the selection functions would only consider those from the module.

In this direction, this section analyses a few cases where one can find isomorphic pairs of selection functions $\mathrm{g}$ w.r.t. $\bmod (\mathcal{O}, \Omega, \Phi)$ easily. In these instances, there is no need for explicit references to $\mathbf{g}$, or $\mathcal{O}$ (unless via the module). The first of these corresponds to the full meet selection, which always selects all the MaxNons [AGM85].

Remark 4.1.10. Let $\mathcal{M}=\bmod (\mathcal{O}, \Omega, \Phi)$ be a JPM for a finite DL-setting $(\mathfrak{L}, \mathcal{O}, \Omega, \Phi)$. Also, for simplicity consider that $\operatorname{Cn}(\Phi) \cap \Omega=\emptyset$, otherwise the three types of selection functions would behave differently. Then:

$$
\mathbf{g}_{\text {full }}(\operatorname{MaxNons}(\mathcal{O}, \Omega, \Phi), \Omega, \Phi)=\operatorname{MaxNons}(\mathcal{O}, \Omega, \Phi)
$$

and

$$
\left.\mathbf{g}_{\text {full }}^{\prime}(\operatorname{MaxNons}(\mathcal{M}, \Omega, \Phi), \Omega, \Phi)=\operatorname{MaxNons}(\mathcal{M}, \Omega, \Phi)\right]
$$

are isomorphic w.r.t. $\mathcal{M}$.

The isomorphism in the previous remark is a direct consequence of Proposition 4.1.3. Additionally, full meet is also related to the smallest MinImp-preserving module of an ontology: $\bigcup \operatorname{MinImps}(\mathcal{O}, \Omega, \Phi)$ because $\bigcap \operatorname{MaxNons}(\mathcal{O}, \Omega, \Phi)=\mathcal{O} \backslash \bigcup \operatorname{MinImps}(\mathcal{O}, \Omega, \Phi)$. Note that an isomorphic counterpart for full meet selection can be obtained straightforwardly for any JPM.

Another interesting function for which one can generate an isomorphic selection function directly is $\mathbf{g}_{\text {card }}$. This function selects the MaxNons of maximum cardinality. The argument for $\mathbf{g}_{\text {card }}$ is that, Proposition 4.1.6 entails that $|X \cap \mathcal{M}|<\left|X^{\prime} \cap \mathcal{M}\right|$ iff $|X|<\left|X^{\prime}\right|$, for $X, X^{\prime} \in \operatorname{MaxNons}(\mathcal{O}, \Omega, \Phi)$, and it is always hold that $\mathcal{M} \subseteq \bigcup \operatorname{MinImps}(\mathcal{O}, \Omega, \Phi)$. Essentially, the isomorphic versions are easy to obtain because the same number of axioms is removed from each module upon modularisation.

More generally, suppose that there is a strict preorder with minimal elements $\prec$ defined over $\operatorname{MaxNons}(\mathcal{O}, \Omega, \Phi)$ and that $\mathbf{g}$ is a selection function defined as $\mathbf{g}(\operatorname{MaxNons}(\mathcal{O}, \Omega, \Phi), \Omega, \Phi)=$ $\max _{\prec}(\operatorname{Max} \operatorname{Nons}(\mathcal{O}, \Omega, \Phi))$, when $\operatorname{Cn}(\Phi) \cup \Omega=\emptyset$. If for $\mathcal{M} \subseteq \bigcup \operatorname{MinImps}(\mathcal{O}, \Omega, \Phi) A \cap \mathcal{M} \prec^{\prime} B \cap \mathcal{M}$ iff $A \prec B$, then $\mathbf{g}_{\prec^{\prime}}^{\prime}(\operatorname{MaxNons}(\mathcal{M}, \Omega, \Phi), \Omega, \Phi)=\max _{\prec^{\prime}}(\operatorname{MaxNons}(\mathcal{M}, \Omega, \Phi))$ is isomorphic to $\mathbf{g}$ w.r.t. $\mathcal{M}$. This definition is useful whenever such $\prec$ is explicit and $\prec^{\prime}$ easily determined. 
Another method to obtain isomorphic functions is via decomposition. If one can decompose $\mathbf{g}$ into smaller selection functions and find an isomorphic version for each w.r.t. the same module, then their union is an isomorphic pair for $\mathrm{g}$ w.r.t. this same module. Lemma 4.1.11 supports this intuitive notion by showing that selection functions over the same domain are closed under union.

Lemma 4.1.11. Let $\left\{\mathbf{g}_{i}\right\}_{i \in I \subseteq \mathbb{N}}$ be a finite set of selection functions of the same type (i.e. all without protection, all protecting any input or all protecting consistent inputs) for $\mathcal{O}, \Omega$ and $\Phi$, then $\mathbf{g}$ defined as: $\mathbf{g}(\mathcal{O}, \Omega, \Phi)=\bigcup_{i \in I} \mathbf{g}_{i}(\mathcal{O}, \Omega, \Phi)$ is also a selection function for $\mathcal{O}, \Omega$ and $\Phi$.

Proof. Let $\mathbf{g}$ be a selection function as stated. If $\operatorname{MaxNons}(\mathcal{O}, \Omega, \Phi)=\operatorname{MaxNons}\left(\mathcal{O}^{\prime}, \Omega\right.$, $\left.\Phi\right)$, then $\mathbf{g}(\operatorname{MaxNons}(\mathcal{O}, \Omega, \Phi), \Omega, \Phi)=\mathbf{g}\left(\operatorname{MaxNons}\left(\mathcal{O}^{\prime}, \Omega, \Phi\right), \Omega, \Phi\right)$ because the same equality holds for each $\mathbf{g}_{i}$ since each is a function. Also, whenever $\operatorname{MaxNons}(\mathcal{O}, \Omega, \Phi) \neq \emptyset$ we have for all $i \in I$ :

$$
\emptyset \neq \mathbf{g}_{i}(\operatorname{MaxNons}(\mathcal{O}, \Omega, \Phi), \Omega, \Phi) \subseteq \operatorname{MaxNons}(\mathcal{O}, \Omega, \Phi)
$$

and thus,

$$
\emptyset \neq \mathbf{g}(\operatorname{MaxNons}(\mathcal{O}, \Omega, \Phi), \Omega, \Phi) \subseteq \operatorname{MaxNons}(\mathcal{O}, \Omega, \Phi) .
$$

Otherwise, since each $\mathbf{g}_{i}(\operatorname{MaxNons}(\mathcal{O}, \Omega, \Phi), \Omega, \Phi)=\{\mathcal{O}\}, \mathbf{g}(\operatorname{MaxNons}(\mathcal{O}, \Omega, \Phi), \Omega, \Phi)=\mathcal{O}$.

As the only properties of selection functions used in this proof are the domains and the fact that they are functions, this result holds for all three types of selection functions (Definitions 2.3.10, 2.3.21 and 2.3.22).

Nevertheless, obtaining a isomorphic restriction for a module is not always straightforward (unless the definition is trivialised as in Equation (4.1)). Example 4.1.12 illustrates this fact with two selection functions based on the signature size of the MaxNons.

Example 4.1.12. Consider the following ontology:

$$
\begin{aligned}
& \mathcal{O}=\{ \\
& \alpha_{1}: \mathrm{A} \sqsubseteq \mathrm{B} \sqcup \mathrm{E}, \quad \alpha_{2}: \mathrm{A} \sqsubseteq \mathrm{C}, \\
& \alpha_{3}: \mathrm{B} \sqsubseteq \mathrm{D}, \\
& \alpha_{5}: \mathrm{C} \sqcap \mathrm{D} \sqsubseteq \perp, \alpha_{6}: \exists \mathrm{r} \cdot \mathrm{A} \sqsubseteq \mathrm{F}, \\
& \alpha_{7}: \forall \mathrm{r} . \mathrm{D} \sqsubseteq \mathrm{E}
\end{aligned}
$$

Let $\Phi=\emptyset$ and $\Omega=\{\mathrm{A} \sqsubseteq \mathrm{D}\}$ and suppose that $\bmod$ is a $\mathrm{JPM}$ such that $\bmod (\mathcal{O}, \Omega, \Phi)=\mathcal{M}=$ $\left\{\alpha_{1}, \alpha_{2}, \alpha_{3}, \alpha_{4}\right\}$. Also let $\mathbf{g}$ and $\mathbf{g}^{\prime}$ be selection functions such that:

$$
\begin{gathered}
\mathbf{g}(\operatorname{MaxNons}(\mathcal{O}, \Omega, \Phi), \Omega, \Phi)=\arg \max _{X \in \operatorname{MaxNons}(\mathcal{O}, \Omega, \Phi)}|\operatorname{sig}(X)| \\
\mathbf{g}^{\prime}(\operatorname{MaxNons}(\mathcal{M}, \Omega, \Phi), \Omega, \Phi)=\arg \max _{X \in \bmod (\mathcal{O}, \Omega, \Phi)}|\operatorname{sig}(X)|
\end{gathered}
$$

Consequently:

$$
\begin{aligned}
\mathbf{g}(\operatorname{Max} \operatorname{Nons}(\mathcal{O}, \Omega, \Phi), \Omega, \Phi) & =\left\{\mathcal{O} \backslash \alpha_{i}\right\}_{1 \leq i \leq 4} \\
\mathbf{g}^{\prime}(\operatorname{MaxNons}(\mathcal{M}, \Omega, \Phi), \Omega, \Phi) & =\left\{\mathcal{M} \backslash \alpha_{j}\right\}_{2 \leq j \leq 4}
\end{aligned}
$$

Therefore, $\mathbf{g}$ and $\mathbf{g}^{\prime}$ are not isomorphic.

Using JPMs one can pass $\bmod (\mathcal{O}, \Omega, \Phi)$ instead of $\mathcal{O}$ to Algorithm 1, cutting the search space of the whole algorithm, including the getNode methods which return a MinImp or a MaxNon at each invocation. The next section details an improvement to this approach. Instead of relying on a single module extraction, it provides a method to reduce or increase the module size efficiently during the computation of MinImps. 


\subsection{Locality-Based Modules Incremental Modularity Bounds}

As discussed in Section 2.4, there are module approaches, such as the syntactic locality-based modules, that provide more logical properties than justification-preservation. This section shows these additional properties allows us to provide even smaller modules while computing MinImps. The same approach is studied for MaxNons, but another asymmetry rises, showing that the result for MaxNon does not improve single module extraction (SME) strategies.

For the reasons pointed out in Subsection 2.4.3, this thesis focuses on LBMs. Moreover, while the three main types of locality-based modules $\left(\perp, T\right.$ and $\left.T \perp^{*}\right)$ satisfy all properties needed throughout this Section 4.2, $\top \perp^{*}$-LBMs receive special attention. These modules are favoured because they are (potentially) smaller than their counterparts. Furthermore, results employ the properties unique to these nested modules. As a drawback, when compared to $\perp$ and T-LBMs, the proofs get more complicated (one reason why Subsection 2.4.3 introduces the notion of extraction sequences via Definition 2.4.9).

Before proceeding, note that there is a mismatch between the definition of MinImp-preserving modules (Proposition 4.1.1) and that of LBMs (Definition 2.4.4). The first defines modules for sets of formulas, while the other defines modules for a signature. However, as mentioned Subsection 2.4.3, such differece is easy to solve: whenever one needs $\bmod (\mathcal{O}, \Omega, \Phi)$, then $x-\operatorname{LBM}(\mathcal{O}, \operatorname{sig}(\Omega \cup \Phi)) \cap \mathcal{O})$ is a suitable module.

The intuitive idea consists in updating the initial module $\bmod (\mathcal{O}, \Omega, \Phi)$ at each call of get Node (Line 8 of Algorithm 1). The naïve approach would be to recompute the module from scratch at each iteration. However that would increase the overhead of modularity significantly.

Thus, the next results aim to determine how to transform the module considering the path of the hitting set tree (henceforth, hitting path) that is being examined in the current iteration. Ideally, this partial reuse would save many locality-check operations considering that, with either FIFO or LIFO policies, subsequent calls get Node are most of the times similar.

Unfortunately, the most important results in this section (e.g. Corollaries 4.2.3 and 4.2.4) only aid MinImps' computation. For MinImps, a hitting path $P$ indicates the axioms that need to be deleted from the ontology before looking for a MinImp, in this way, the larger the hitting path, smaller the module. For MaxNons, the hitting path is a lower bound (the argument $L$ in Algorithm 5): the MaxNon must contain all axioms in the hitting path. Since the initial module corresponds to the hitting path $P=\emptyset$, whenever one forces the module to include all axioms in $P^{\prime} \supset$ $P$ then, as consequence of monotonicity on ontology and signature enlargements (Proposition 2.4.8), the resulting module is bigger than the starting one.

When computing MinImps with Algorithm 1, the hitting path indicates the axioms that have to be removed from the ontology, before looking for the next MinImp. Hence, before calling get Node, the ontology can be replaced by the $\operatorname{module} \bmod (\mathcal{O} \backslash P, \Omega, \Phi)$.

Suppose now, that the $P$ was the hitting path in the previous iteration, and that now $P^{\prime}$ is the hitting path for the current one. Then, the procedure needs to change $\bmod (\mathcal{O} \backslash P, \Omega, \Phi)$ into $\bmod \left(\mathcal{O} \backslash P^{\prime}, \Omega, \Phi\right)$. The transition from one module to the other can be made in two steps, first shrinking the current module, removing unneeded axioms, and later expanding the result to account for potential additions.

Proposition 4.2.1 uses the monotonicity properties from Proposition 2.4.8 to convert $x$ - $\operatorname{LBM}(\mathcal{O} \backslash$ $P, \Sigma)$ into $x$ - $\operatorname{LBM}\left(\mathcal{O} \backslash\left(P^{\prime} \cup P\right), \Sigma\right)$. This transformation shrinks the module, giving a lower approximation of the module $x-\operatorname{LBM}\left(\mathcal{O} \backslash P^{\prime}, \Sigma\right)$, needed for the current path $P^{\prime}$.

Proposition 4.2.1. Let $\mathcal{O}$ be an ontology, $A, B \subseteq \mathcal{O}, \Sigma$ a signature and $x \in\left\{\perp, \top, \top \perp^{*}\right\}$ a $L B M$ type. Then:

$$
x-\operatorname{LBM}(\mathcal{O} \backslash(A \cup B), \Sigma)=x-\operatorname{LBM}(x-\operatorname{LBM}(\mathcal{O} \backslash A, \Sigma) \backslash B, \Sigma)
$$


Proof. $x-\operatorname{LBM}(x-\operatorname{LBM}(\mathcal{O} \backslash A, \Sigma) \backslash B, \Sigma) \subseteq x-\operatorname{LBM}(\mathcal{O} \backslash(A \cup B), \Sigma):$

$$
\begin{aligned}
& x-\operatorname{LBM}(\mathcal{O} \backslash A, \Sigma) \subseteq(\mathcal{O} \backslash A) \\
& x-\operatorname{LBM}(\mathcal{O} \backslash A, \Sigma) \backslash B \subseteq(\mathcal{O} \backslash A) \backslash B \\
& x-\operatorname{LBM}(x-\operatorname{LBM}(\mathcal{O} \backslash A, \Sigma) \backslash B, \Sigma) \subseteq x-\operatorname{LBM}(\mathcal{O} \backslash(A \cup B), \Sigma) \\
& x-\operatorname{LBM}(\mathcal{O} \backslash(A \cup B), \Sigma) \subseteq x-\operatorname{LBM}(x-\operatorname{LBM}(\mathcal{O} \backslash A, \Sigma) \backslash B, \Sigma): \\
& x-\operatorname{LBM}((\mathcal{O} \backslash A) \backslash B, \Sigma) \subseteq x-\operatorname{LBM}(\mathcal{O} \backslash A, \Sigma) \\
& x-\operatorname{LBM}((\mathcal{O} \backslash A) \backslash B, \Sigma) \backslash B \subseteq x-\operatorname{LBM}(\mathcal{O} \backslash A, \Sigma) \backslash B \\
& x-\operatorname{LBM}(x-\operatorname{LBM}((\mathcal{O} \backslash A) \backslash B, \Sigma), \Sigma) \subseteq x-\operatorname{LBM}(x-\operatorname{LBM}(\mathcal{O} \backslash A, \Sigma) \backslash B, \Sigma) \quad \text { (Proposition 2.4.8(2)) } \\
& x-\operatorname{LBM}(\mathcal{O} \backslash(A \cup B), \Sigma) \subseteq x-\operatorname{LBM}(x-\operatorname{LBM}(\mathcal{O} \backslash A, \Sigma) \backslash B, \Sigma) \quad \text { (Proposition 2.4.8(1)) }
\end{aligned}
$$

In this way, one can remove axioms and restore the set as a module simply by doing:

$$
x-\operatorname{LBM}\left(\mathcal{O} \backslash\left(P \cup P^{\prime}\right), \Sigma\right)=x-\operatorname{LBM}\left(x-\operatorname{LBM}(\mathcal{O} \backslash P, \Sigma) \backslash P^{\prime}, \Sigma\right)
$$

Some axioms could be in $P$, but not in $P^{\prime}$. Thus, there could be axioms in the current module that did not belong to the previous one. A straightforward alternative to recover them is to restore initial module because Proposition 4.1.1 and Proposition 4.1.3 already prove that the initial one is sufficient. Still, this would bring back axioms that should have been excluded from the hitting path, and others that would become non-local because of them.

Instead of the solution above, one can start with the reduced module obtained via Proposition 4.2.1 and then add to it just the axioms required to obtain the module for the next call to get Node $\left(\bmod \left(\mathcal{O} \backslash P^{\prime}, \Omega, \Phi\right)\right)$. The intuitive idea consists in extracting a module from the axioms that are not in the reduced module, using a suitable signature.

Theorem 4.2.2 shows a result that is valid for any extraction sequence (as in Definition 2.4.9). This theorem will facilitate the proof of results for LBMs in general, but in particular for $T \perp^{*}$-LBMs.

Theorem 4.2.2. Let $\mathcal{O}$ be an ontology, $\Sigma$ and $\Sigma^{\prime}$ signatures with $\Sigma \subseteq \Sigma^{\prime}$, and $R \subseteq \mathcal{O}$. Then:

$$
\begin{gathered}
(x)_{n}-\operatorname{LBM}(\mathcal{O}, \Sigma)=(x)_{n}-\operatorname{LBM}\left(\left(\mathcal{O} \backslash \top \perp^{*}-\operatorname{LBM}(\mathcal{O} \backslash R, \Sigma)\right), \Sigma \cup \operatorname{sig}\left(\top \perp^{*}-\operatorname{LBM}(\mathcal{O} \backslash R, \Sigma)\right)\right) \\
\cup \top \perp^{*}-\operatorname{LBM}(\mathcal{O} \backslash R, \Sigma)
\end{gathered}
$$

Proof. The proof uses induction on $n$, the length of the extraction sequence. To shorten the formulas in this demonstration, $\mathcal{M}_{(\Sigma, R)}^{*}$ will be used in place of $\top \perp^{*}-\operatorname{LBM}(\mathcal{O} \backslash R, \Sigma)$.

Base case: $n=1$ In this case: $(x)_{n}-\operatorname{LBM}(\mathcal{O}, \Sigma)=x_{1}-\operatorname{LBM}(\mathcal{O}, \Sigma)$ and $(x)_{n}-\operatorname{LBM}\left(\left(\mathcal{O} \backslash \mathcal{M}_{(\Sigma, R)}^{*}\right), \Sigma \cup\right.$ $\left.\operatorname{sig}\left(\mathcal{M}_{(\Sigma, R)}^{*}\right)\right) \cup \mathcal{M}_{(\Sigma, R)}^{*}=x_{1}-\operatorname{LBM}\left(\left(\mathcal{O} \backslash \mathcal{M}_{(\Sigma, R)}^{*}\right), \Sigma \cup \operatorname{sig}\left(\mathcal{M}_{(\Sigma, R)}^{*}\right)\right) \cup \mathcal{M}_{(\Sigma, R)}^{*}$ for $x_{1} \in\{\top, \perp\}$. Note that Corollary 2.4.11 guarantees that $\mathcal{M}_{(\Sigma, R)}^{*} \subseteq x_{1}-\operatorname{LBM}(\mathcal{O}, \Sigma)$. Also, due monotonicity on ontology and signature enlargements (Proposition 2.4.8) and self-containment (Definition 2.4.7) of $x$-LBMs: $x_{1}-\operatorname{LBM}\left(\left(\mathcal{O} \backslash \mathcal{M}_{(\Sigma, R)}^{*}\right), \Sigma \cup \operatorname{sig}\left(\mathcal{M}_{(\Sigma, R)}^{*}\right)\right)$

$$
\begin{aligned}
& \subseteq x_{1}-\operatorname{LBM}\left(\mathcal{O}, \Sigma \cup \operatorname{sig}\left(\mathcal{M}_{(\Sigma, R)}^{*}\right)\right) \\
& \subseteq x_{1}-\operatorname{LBM}\left(\mathcal{O}, \Sigma \cup \operatorname{sig}\left(x_{1}-\operatorname{LBM}(\mathcal{O}, \Sigma)\right)\right) \\
& =x_{1}-\operatorname{LBM}(\mathcal{O}, \Sigma)
\end{aligned}
$$

Thus, $x_{1}-\operatorname{LBM}\left(\left(\mathcal{O} \backslash \mathcal{M}_{(\Sigma, R)}^{*}\right), \Sigma \cup \operatorname{sig}\left(\mathcal{M}_{(\Sigma, R)}^{*}\right)\right) \cup \mathcal{M}_{(\Sigma, R)}^{*} \subseteq x_{1}-\operatorname{LBM}(\mathcal{O}, \Sigma)$.

Now for the converse inclusion. If $\alpha \in x_{1}-\operatorname{LBM}(\mathcal{O}, \Sigma)$ then $\alpha \in x-\mathrm{NL}_{i}(\mathcal{O}, \Sigma)$ for some $i \in \mathbb{N}$. Next, it is proved by induction on $i$ that $x-\mathrm{NL}_{i}(\mathcal{O}, \Sigma) \subseteq x_{1}-\operatorname{LBM}(\mathcal{O}, \Sigma)$. 
Base case: $(i=0)$ If $\alpha \in \mathcal{M}_{(\Sigma, R)}^{*}$ the proof the result is trivially valid. Otherwise, since $\alpha \in x-\mathrm{NL}_{0}(\mathcal{O}, \Sigma), \alpha$ is not $x$-local w.r.t. $\Sigma$. Further, as $\alpha \in\left(\mathcal{O} \backslash \mathcal{M}_{(\Sigma, R)}^{*}\right)$, Remark 2.4.12 implies that $\alpha \in x_{1}-\operatorname{LBM}\left(\left(\mathcal{O} \backslash \mathcal{M}_{(\Sigma, R)}^{*}\right), \Sigma \cup \operatorname{sig}\left(\mathcal{M}_{(\Sigma, R)}^{*}\right)\right)$.

Induction step: (IH: the inclusion holds for $k-1$ ) If $\alpha \in \mathcal{M}_{(\Sigma, R)}^{*}$ the result immediately holds. Otherwise, recall that as $\alpha \in x-\mathrm{NL}_{k}(\mathcal{O}, \Sigma), \alpha$ is not $x$-local w.r.t. the signature $\Sigma \cup \operatorname{sig}\left(x-\mathrm{NL}_{k-1}(\mathcal{O}, \Sigma)\right)$. According to the induction hypothesis, $x-\mathrm{NL}_{k-1} \subseteq x_{1}-\mathrm{LBM}((\mathcal{O} \backslash$ $\left.\left.\mathcal{M}_{(\Sigma, R)}^{*}\right), \Sigma \cup \operatorname{sig}\left(\mathcal{M}_{(\Sigma, R)}^{*}\right)\right) \cup \mathcal{M}_{(\Sigma, R)}^{*}$. Hence $\alpha$ is also non- $x$-local w.r.t. $\operatorname{sig}\left(x_{1}-\operatorname{LBM}((\mathcal{O} \backslash\right.$ $\left.\left.\left.\mathcal{M}_{(\Sigma, R)}^{*}\right), \Sigma \cup \operatorname{sig}\left(\mathcal{M}_{(\Sigma, R)}^{*}\right)\right) \cup \mathcal{M}_{(\Sigma, R)}^{*}\right)=\operatorname{sig}\left(x_{1}-\operatorname{LBM}\left(\left(\mathcal{O} \backslash \mathcal{M}_{(\Sigma, R)}^{*}\right), \Sigma \cup \operatorname{sig}\left(\mathcal{M}_{(\Sigma, R)}^{*}\right)\right)\right)$. Then, Remark 2.4.12 implies that $\alpha \in x_{1}-\operatorname{LBM}\left(\left(\mathcal{O} \backslash \mathcal{M}_{(\Sigma, R)}^{*}\right), \Sigma \cup \operatorname{sig}\left(\mathcal{M}_{(\Sigma, R)}^{*}\right)\right)$.

Thus, it holds that $x_{1}-\operatorname{LBM}\left(\left(\mathcal{O} \backslash \mathcal{M}_{(\Sigma, R)}^{*}\right), \Sigma \cup \operatorname{sig}\left(\mathcal{M}_{(\Sigma, R)}^{*}\right)\right) \cup \mathcal{M}_{(\Sigma, R)}^{*} \subseteq x_{1}-\operatorname{LBM}(\mathcal{O}, \Sigma)$.

Induction step: (IH: the equality holds for $n-1$ ) First, consider the relation below, which will figure in the final steps of the proof.

$$
\begin{aligned}
\mathcal{M}_{(\Sigma, R)}^{*} & \subseteq x_{n^{-}} \operatorname{LBM}\left(\mathcal{M}_{(\Sigma, R)}^{*}, \Sigma\right) \\
& \subseteq x_{n^{-}} \operatorname{LBM}\left((x)_{n-1}-\operatorname{LBM}\left(\left(\mathcal{O} \backslash \mathcal{M}_{(\Sigma, R)}^{*}\right), \Sigma \cup \operatorname{sig}\left(\mathcal{M}_{(\Sigma, R)}^{*}\right)\right)\right. \\
& \left.\cup \mathcal{M}_{(\Sigma, R)}^{*}, \Sigma\right)
\end{aligned}
$$

Now, one can combine the definition of extraction sequence (Definition 2.4.9), the induction hyphothesis and the relation (4.3) to prove the induction step.

$$
\begin{aligned}
(x)_{n}-\operatorname{LBM}(\mathcal{O}, \Sigma)= & x_{n}-\operatorname{LBM}\left((x)_{n-1}-\operatorname{LBM}(\mathcal{O}, \Sigma), \Sigma\right) \\
= & x_{n}-\operatorname{LBM}\left((x)_{n-1}-\operatorname{LBM}\left(\left(\mathcal{O} \backslash \mathcal{M}_{(\Sigma, R)}^{*}\right),\right.\right. \\
& \left.\left.\quad \Sigma \cup \operatorname{sig}\left(\mathcal{M}_{(\Sigma, R)}^{*}\right)\right) \cup \mathcal{M}_{(\Sigma, R)}^{*}, \Sigma\right) \\
= & x_{n}-\operatorname{LBM}\left((x)_{n-1}-\operatorname{LBM}\left(\left(\mathcal{O} \backslash \mathcal{M}_{(\Sigma, R)}^{*}\right),\right.\right. \\
& \left.\left.\quad \Sigma \cup \operatorname{sig}\left(\mathcal{M}_{(\Sigma, R)}^{*}\right)\right) \cup \mathcal{M}_{(\Sigma, R)}^{*}, \Sigma \cup \operatorname{sig}\left(\mathcal{M}_{(\Sigma, R)}^{*}\right)\right) \\
= & x_{n}-\operatorname{LBM}\left((x)_{n-1}-\operatorname{LBM}\left(\left(\mathcal{O} \backslash \mathcal{M}_{(\Sigma, R)}^{*}\right),\right.\right. \\
& \left.\left.\quad \Sigma \cup \operatorname{sig}\left(\mathcal{M}_{(\Sigma, R)}^{*}\right)\right), \Sigma \cup \operatorname{sig}\left(\mathcal{M}_{(\Sigma, R)}^{*}\right)\right) \cup \mathcal{M}_{(\Sigma, R)}^{*} \\
= & (x)_{n}-\operatorname{LBM}\left(\left(\mathcal{O} \backslash \mathcal{M}_{(\Sigma, R)}^{*}\right), \Sigma \cup \operatorname{sig}\left(\mathcal{M}_{(\Sigma, R)}^{*}\right)\right) \cup \mathcal{M}_{(\Sigma, R)}^{*}
\end{aligned}
$$

Hence, theorem holds for any extraction sequence, regards of its length.

Finally, Corollary 4.2.3 provides the main result that will allow us to calculate $T \perp^{*}-\operatorname{LBM}(\mathcal{O} \backslash$ $\left.P^{\prime}, \Sigma\right)$ from $\top \perp^{*}-\operatorname{LBM}\left(\mathcal{O} \backslash\left(P \cup P^{\prime}\right), \Sigma\right)$.

Corollary 4.2.3. Let $\mathcal{O}$ be an ontology, $R \subseteq \mathcal{O}$, and $\Sigma$ and $\Sigma^{\prime}$ signatures. Then

$$
\begin{gathered}
\top \perp^{*}-\operatorname{LBM}(\mathcal{O}, \Sigma)=\top \perp^{*}-\operatorname{LBM}\left(\left(\mathcal{O} \backslash \top \perp^{*}-\operatorname{LBM}(\mathcal{O} \backslash R, \Sigma)\right), \Sigma \cup \operatorname{sig}\left(\top \perp^{*}-\operatorname{LBM}(\mathcal{O} \backslash R, \Sigma)\right)\right) \\
\cup \top \perp^{*}-\operatorname{LBM}(\mathcal{O} \backslash R, \Sigma)
\end{gathered}
$$


Proof. Direct consequence of Theorem 4.2.2 and Corollary 2.4.11.

Interestingly, for $\perp$ and $T$-LBMs one can replace the $T \perp^{*}$-LBM with the $\perp$-LBM or $T$-LBM, respectively, obtaining analogous results (Corollary 4.2.4).

Corollary 4.2.4. Let $\mathcal{O}$ be an ontology, $R \subseteq \mathcal{O}, \Sigma$ and $\Sigma^{\prime}$ signatures and $x \in\{\perp, \top\}$ a locality notion. Then

$$
\begin{gathered}
\top \perp^{*}-\operatorname{LBM}(\mathcal{O}, \Sigma)=x-\operatorname{LBM}((\mathcal{O} \backslash x-\operatorname{LBM}(\mathcal{O} \backslash R, \Sigma)), \Sigma \cup \operatorname{sig}(x-\operatorname{LBM}(\mathcal{O} \backslash R, \Sigma))) \\
\cup x-\operatorname{LBM}(\mathcal{O} \backslash R, \Sigma)
\end{gathered}
$$

Proof. Sketch: similar to the proof of the base case for Theorem 4.2.2, relying on the properties stated in Proposition 2.4.8 and Definition 2.4.7. In an extraction sequence of length 1 , the $x_{1}$-LBM is sufficient where the $T \perp^{*}$-LBM was needed.

Corollaries 4.2.3 and 4.2.4 confirm that one can reuse modules from a previous iteration when axioms need to be added. If one considers $T \perp^{*}-\operatorname{LBM}\left(\mathcal{O} \backslash\left(P^{\prime} \cup P\right), \Sigma\right)$ given, then Corollary 4.2.3 allows the transformation of this module into $\left.\top \perp^{*}-\operatorname{LBM}\left(\mathcal{O} \backslash P^{\prime}\right), \Sigma\right)$ by taking $R=P \backslash P^{\prime}$, while saving locality-checks.

$$
\begin{aligned}
\left.\top \perp^{*}-\operatorname{LBM}\left(\mathcal{O} \backslash P_{\text {cur }}\right), \Sigma\right)=\top & \perp^{*}-\operatorname{LBM}\left(\left(\mathcal{O} \backslash \top \perp^{*}-\operatorname{LBM}\left(\mathcal{O} \backslash\left(P_{\text {prev }} \cup P_{\text {cur }}\right), \Sigma\right)\right),\right. \\
& \left.\Sigma \cup \operatorname{sig}\left(\top \perp^{*}-\operatorname{LBM}\left(\mathcal{O} \backslash\left(P_{\text {prev }} \cup P_{\text {cur }}\right), \Sigma\right)\right)\right) \\
& \cup \top \perp^{*}-\operatorname{LBM}\left(\mathcal{O} \backslash\left(P_{\text {prev }} \cup P_{\text {cur }}\right), \Sigma\right)
\end{aligned}
$$

This equality and the results in this section underpin Algorithm 13. This algorithm computes MinImps using locality-based modules as upper bounds, while avoiding computing them from scratch every time.

Note that the SME approaches and Algorithm 13 still maintain the same complexity in the worst case as the non-modularised versions of MaxNons and MinImps computation. Still, the modularised versions (potentially) make fewer reasoner calls because they reduce the search space considered when building the hitting set tree (HST) and computing each node. 


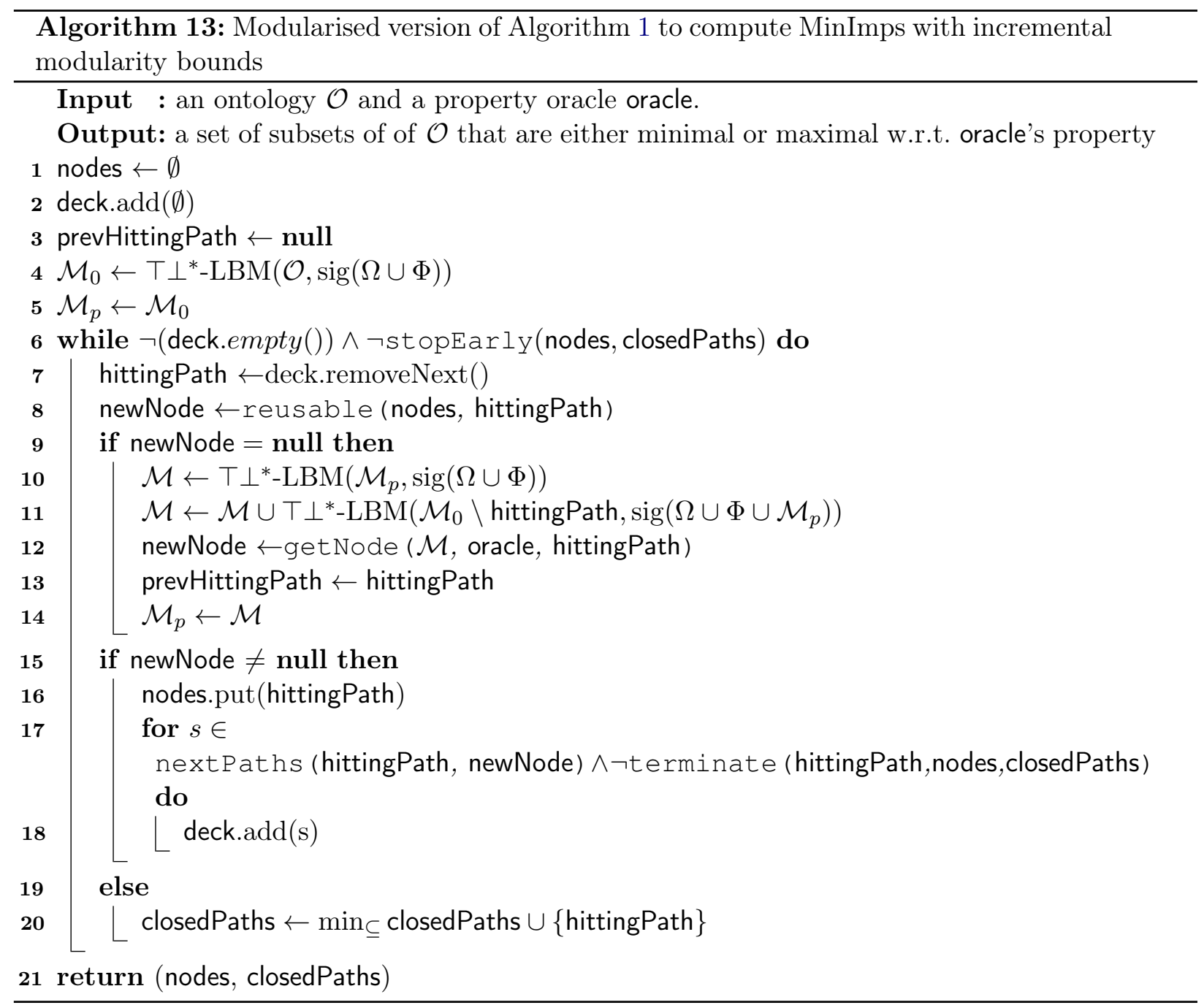




\section{Chapter 5}

\section{Experimental Framework}

This chapter details the empirical framework tailored for the experiments which address the second research question (Q2), presented in Section 3.3. It covers the datasets selected, the quantities evaluated and the methods used to collect the relevant metrics. A whole section is dedicated to a novel (albeit prototypical) tool to generate benchmark inputs for Ontology Repair (and Belief Change) methods. Further, it briefly discusses potential issues and countermeasures when assessing performance in terms of execution time and memory consumed.

\subsection{Corpus Generator for Belief Change and Ontology Repair}

Obtaining a suitable corpus for benchmarking Belief Change operations is a challenge on its own. The same happens with Ontology Debug and Repair as well as related areas such as Nonmonotonic Reasoning. Not only that, the literature shows a range of distinct experimental designs in this area.

When comparing algorithms that process ontologies, some studies [SQJH08, Nor11, MMS14, BFPS15] focus on a few hand-picked or tailored ontologies. Matentzoglu [Mat16] relies on a normalised snapshot of the BioPortal service while Cóbe and Wassermann [CW15] use both BioPortal and artificially generated ontologies (filtering them according to some criteria such as OWL 2 Profile and ability to process within a specified timeout).

The importance of having a good corpus in this area becomes evident when one looks at the corpus assemblage and curation done by Matentzoglu, Bail and Parsia [MBP13a]. In that work, the authors approach the problem of producing an ontology corpus for a wide range of applications, reasoner benchmarking, for instance.

In most studies mentioned before, the ontology was used "as it is", save for a degree of preprocessing. However, using ontologies as they are provided is not always adequate for the task at hand. For instance, to evaluate their defeasible reasoner, Bonatti et al. [BFPS15] introduce axioms in the Gene Ontology to deliberately cause inconsistencies, forcing their reasoner to perform defeasible inferences.

In contrast with classification and inconsistent checking, benchmarking Belief Change or Ontology Repair operations requires an additional set of formulas as input. Horridge [Hor11] and Cóbe and Wassermann [CW15] focus mostly on implicit subsumptions between concept names on each ontology. One argument for this approach is that they are the first kind of entailment that a user looks at when using ontology editors such as Protégé ${ }^{1}$. Moreover, they can be found in nearly every ontology, even the ones in OWL 2 EL. Another common approach is to focus on solving inconsistencies or unsatisfiable concepts which are fairly universal faults [SC03, KPSG06].

Although there are good examples of ontology datasets, there is not a standard methodology on how to obtain inputs from them for Belief Change (or Ontology Repair). Further, applicationspecific solutions are generally harder to extend in case one aims to include more ontologies or more

\footnotetext{
${ }^{1}$ https://protege.stanford.edu/
} 
input patterns. Mainly on the latter case, such extension might require changing the source code of the benchmark (or even of the whole experiment).

The issues mentioned before motivated the development of a tool to aid the development of reproducible, adaptable and well-specified corpus for Ontology Repair and similar areas. The central idea is to provide separation between the generation of test instances from the experiments themselves and also between the ontologies used as input and the test cases planned. Moreover, as a desideratum, users should be able to write test case specifications in a format that is easy to extend and share.

These requirements led to the development of a prototypical corpus generator for Ontology Repair and Belief Base Change of OWL 2 DL ontologies: the OWL2DL-Change Case Creator (or OWL2DL-CCC). The generator takes as input an ontology and a specification of a test case. It produces as output a set of files representing an instantiation of this test case using the ontology given as input. To produce a corpus for Belief Change, Ontology Repair and other areas a user only needs a set of ontologies, a set of test specification files and the generator. The remaining of this section discusses the components of a test case template, the generation process and the elements of a case instance produced by OWL2DL-CCC.

\subsubsection{Case Specification}

A test case specification is a JSON file containing a predetermined structure. In this file, the user may specify constraints on the input ontology, a set of modifications to the ontology and constraints on the modified (output) ontology. It is divided into blocks or sections: header, term declarations, preconditions, actions, postconditions and catalysts. While this subsection follows this logical sequence of presentation, the blocks can appear in any order in the JSON file.

The first part of the specification is a header containing some metadata about the file that serves as documentation and restricts the profile that the input and output ontologies should be. Listing 5.1 shows an example of this block.

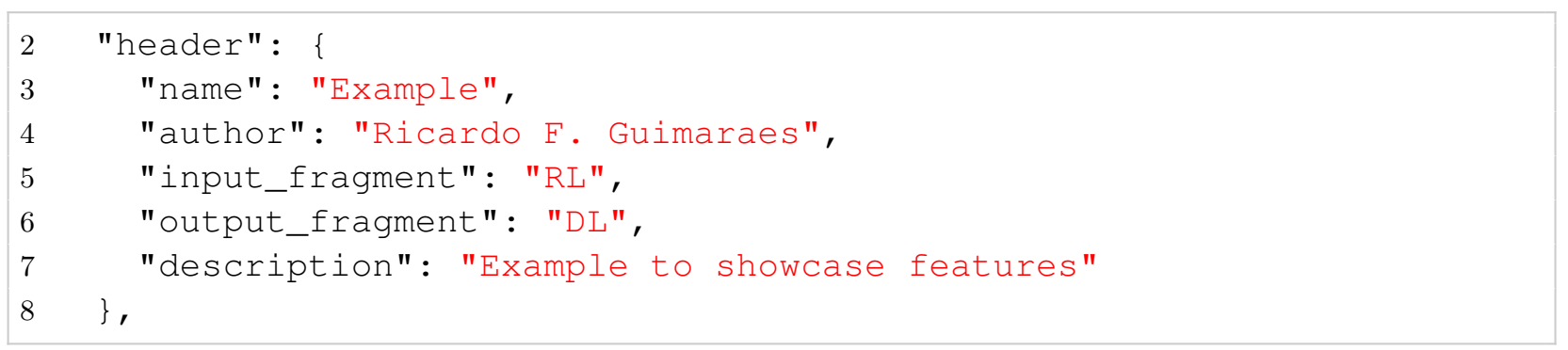

Listing 5.1: Example of a header block

Next comes the term declaration block. This section specifies variables that generator will replace with entities when instantiating the specification for a particular ontology. These variables are used in the remaining blocks to build axioms (which will serve a different purpose in each section they appear in).

These term variables can be constrained according to their type (concept, role, individual) and if they must be fresh or extracted from the ontology's signature. The declaration of terms also has additional parameters for concept variables, including whether they can be $\perp$ or $T$. Moreover, each concept variable can be either primitive, meaning that the generator will replace it with an entity of the ontology, or composite, that is, conjunction or disjunction of primitive concept variables. This block is illustrated with an example in Listing 5.2.

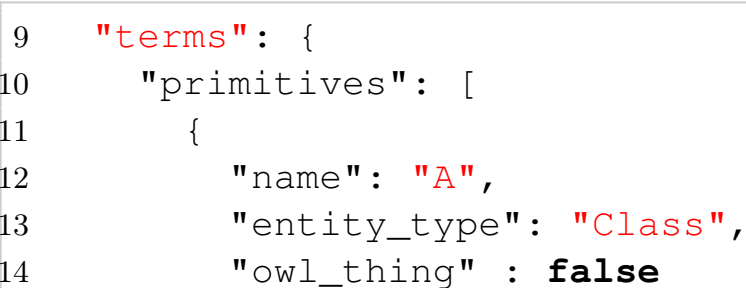




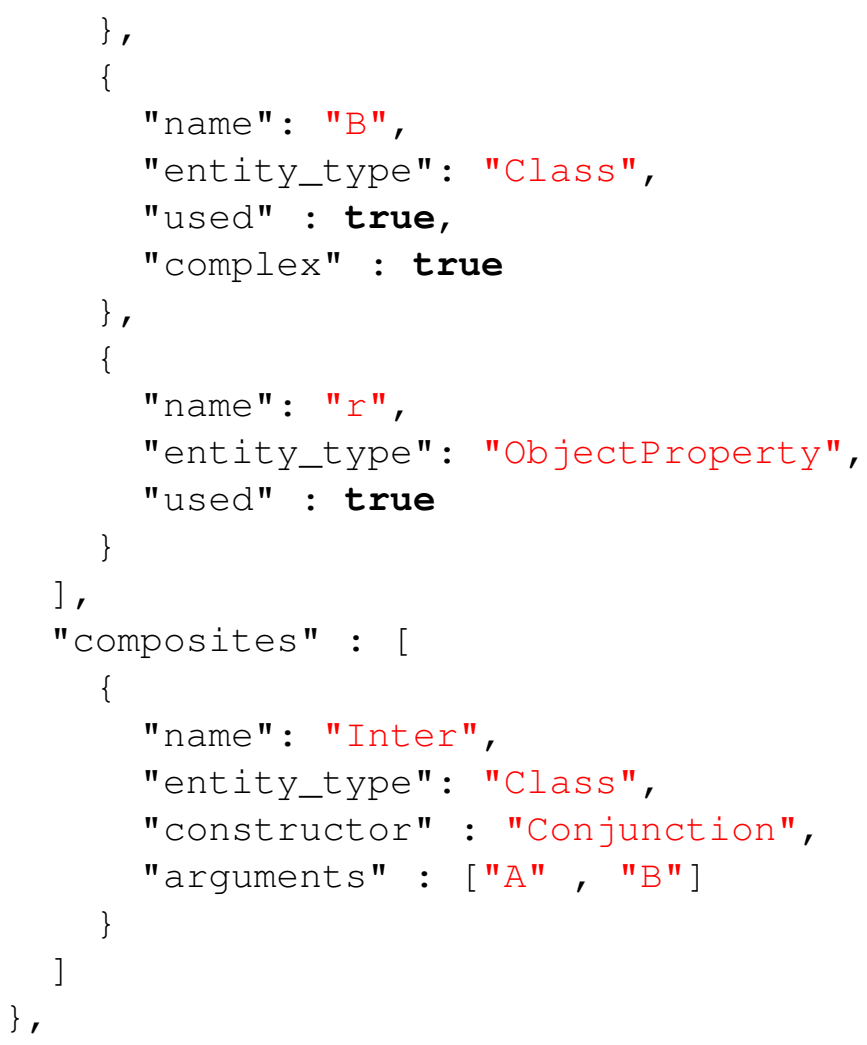

Listing 5.2: Example of a term declaration block

The preconditions block (as illustrated in Listing 5.3) represents the desired constraints on the input ontology. Here the user indicates if it has any restriction on ontology consistency (true or false) or coherence (also true or false). Furthermore, the user can use the variables of the term declarations to write axiom specifications and indicate whether these should be: asserted, entailed, entailed but not asserted or not entailed at all.

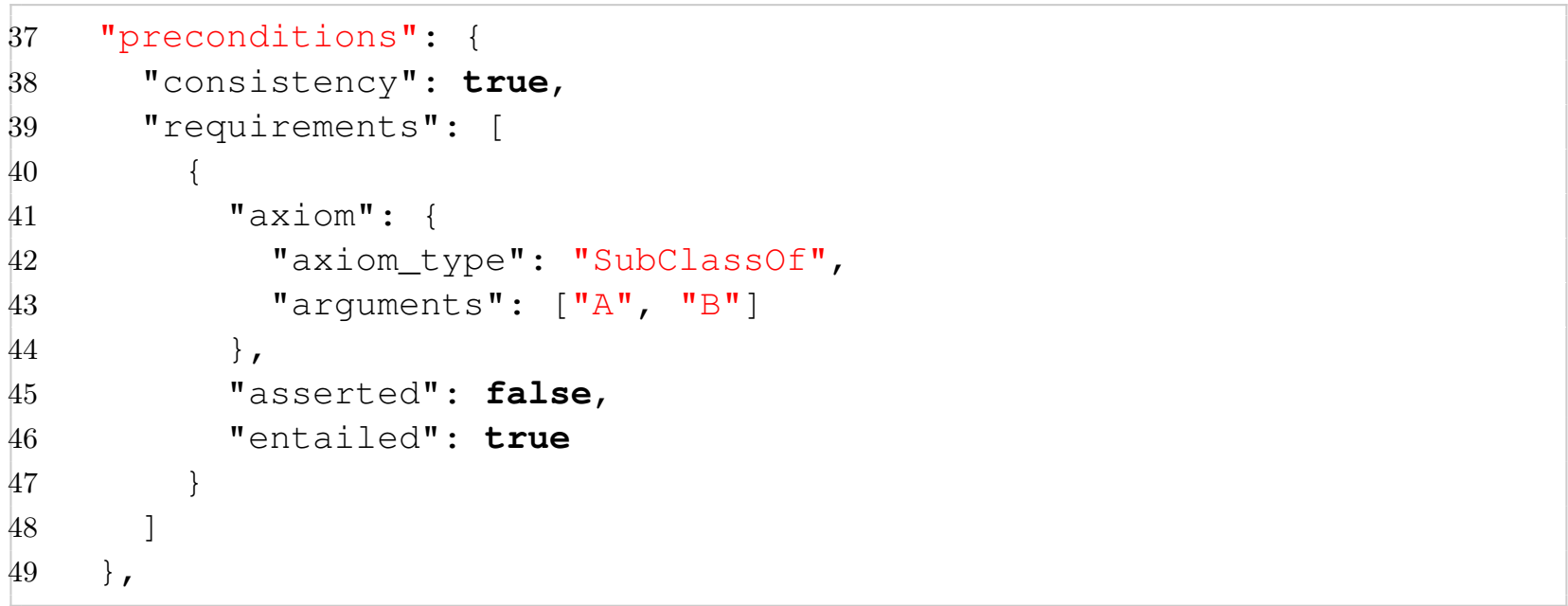

Listing 5.3: Example of a preconditions block

The action block also describes axioms, but instead of placing constraints, these represent either additions or removals of axioms. Currently, the generator can only make explicit inclusions and deletions, that is, only operating with asserted axioms (otherwise there would be circular dependency with the Belief Change problem). For instance, the JSON object in Listing 5.4 forces the addition of an axiom, which states that the domain of the object property attributed to $\mathbf{r}$ is the concept assigned to Inter, to the ontology that will be given as output. 


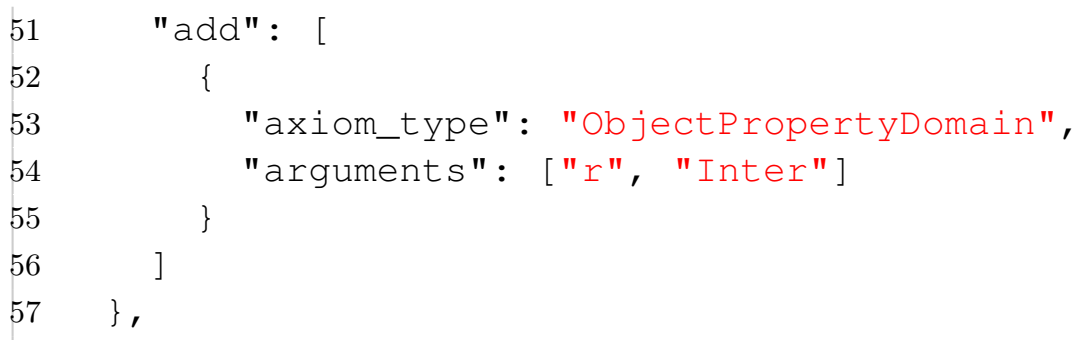

Listing 5.4: Example of an actions block

The postconditions block is analogous to the preconditions one but instead constrains the ontology after the application of the changes in the action block. Listing 5.5 continues the sequence of examples, depicting a simple instance of this block.

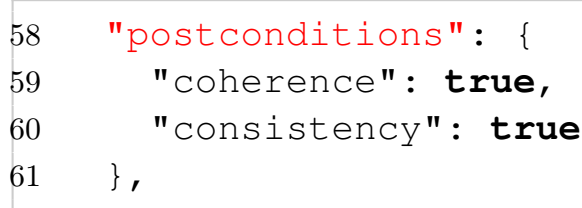

Listing 5.5: Example of a postconditions block

The catalysts section is the most contrived part of the specification. It represents information that is not part of the input ontology nor the output ontology. Still, it contains data that helps to define the input and expectations for each test case. In this part, the user specifies one or more blocks, each with a different name. These blocks may contain the same type of constraints as the preconditions and postconditions blocks. However, the "asserted" information is ignored, and the "entailed" field is used to separate axioms into "positive" or "negative" examples. Listing 5.6 contains an example of this block, which we will discuss next.

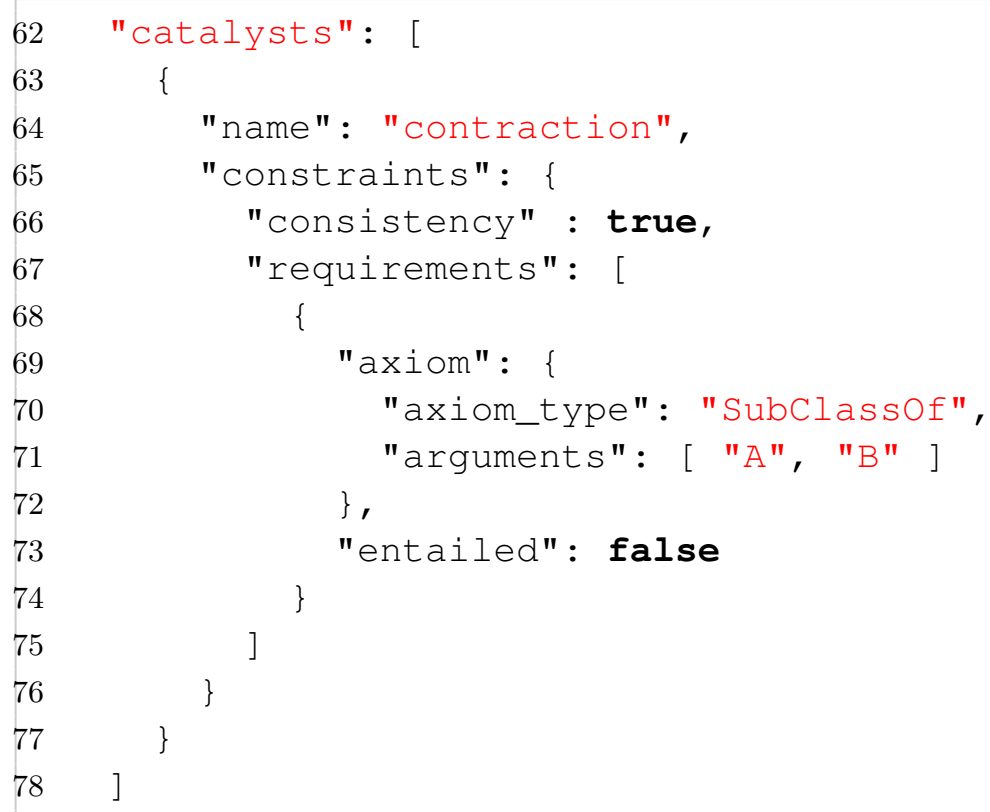

Listing 5.6: Example of a catalysts block

The application which will read the test case is that determines the actual semantics of the elements in the catalyst block that will use the case instance. For example, in the Listing 5.6, the requirement regarding the SubClassOf indicates that the axiom is a negative example. In Belief Change, that may indicate that the axioms should be contracted, or not be present in the output of a contraction. For a reasoning application, it might mean that the inference procedure must not entail such axiom. 


\subsubsection{Instantiation}

Subsection 5.1.1 presented the term declaration block and how this block is used to build the axiom specifications that figure in the other sections of the case specification. This subsection describes how OWL2DL-CCC replaces each term variable that appears in the term declaration block. More specifically, it outlines how the generator searches the ontology's entities for a suitable match to each term variable declared.

The simple answer is that the program uses a brute-force approach: it finds a match for one variable after the other, then it checks all constraints one by one. While OWL2DL-CCC also implements a few heuristics to speed up the search and cut some invalid matches (those that violate the specification) early.

The generator handles primitive term variables first, as once they are determined, so are the composite ones. The primitive term variables are arranged randomly in a list. Then, it iterates over this list, assigning an entity to one term at a time. Whenever one term variable lacks a suitable entity for replacement, the algorithm backtracks to the previous term getting the next entity available to it.

An assignment of an entity to a term variable begins with the selection of candidates among ontology's entities, which is called the domain of the term variable. At first, the domain of each term variable includes every entity of the variable's type (concept, role or individual) in the ontology's signature.

Then, for each axiom requirement in the preconditions block, the generator excludes entities from a term's domain. For instance, if $\mathbf{A}$ is a variable term that must be a subclass of B, its domain is limited to $\mathbf{B}$ 's subclasses, if $\mathbf{B}$ already has a candidate entity. If no candidate is available for A, the algorithm will backtrack until it selects new candidate entities for terms assigned before A (possibly including B). Following, Example 5.1.1 illustrates how the main part of the process instantiation works.

Example 5.1.1. Consider the ontology MovieEx from Example 2.2.1. Let $\mathbf{A}$ and $\mathbf{B}$ be term variables as declared in Listing 5.2. Since $\mathbf{B}$ must be a complex concept, the only options available to replace it are $\exists$ hasScene.SafeScene, SafeScene $\sqcap$ ViolentScene and Trailer $\square \neg$ Movie.

The variable $\mathbf{A}$ can be replaced by any concept as long as it is not $T$, however the precondition in Listing 5.3 will only allow those where $\mathbf{A}$ is an implicit subclass of $\mathbf{B}$.

Note that nothing in instantiation procedures forces the actual ordering of assignment to term variables, so either A or $\mathbf{B}$ could be set first. Without loss of generality, suppose the process assigns Trailer $\sqcap \neg$ Movie to $\mathbf{B}$, then it would constrain the set available options for $\mathbf{A}$ to \{ViolentTeaser\}. Note that Teaser would not be a valid option since the concept inclusion must be implicit.

If OWL2DL-CCC finds a mapping of primitive term variables to entities, it proceeds to the construction entities for the composite term variables (otherwise, the generator exits with a warning to the user). The set of assignments from term variables to entities is called a match. The generator uses these matches is to convert axiom specifications in the preconditions, actions and postconditions blocks into OWL 2 DL axioms. For example, the axiom specification in the action block in Listing 5.4 with a match that replaces $\mathbf{A}$ with ViolentTeaser and $\mathbf{B}$ with Trailer $\sqcap \neg$ Movie would become the axiom ViolentTeaser $\sqsubseteq$ Trailer $\sqcap \neg$ Movie.

With the match computed, the generator verifies whether the input ontology satisfies the axiom requirements in the preconditions. If it does, it creates the axioms from the actions block and applies the required actions. After that, it tests whether the output ontology satisfies the conditions from the postconditions block. Whenever the verification preconditions or postconditions fails, the generator produces a new match, until it is unable to find one.

Finally, if OWL2DL-CCC finds a match that makes the input and output ontologies satisfy all the pre and postconditions, the program generates the output files. A set of output files is assembled for each catalyst, each set includes: the modified ontology, an ontology with axioms 
deemed as positive examples, another with axioms considered negative examples and an JSON file with metadata to aid in reading the test case and reproducing its generation.

\subsubsection{Limitations}

While OWL2DL-CCC still has a considerable margin for improvement, it serves as a proof of concept of what can be achieved towards corpus generation for Belief Change and Ontology Repair.

Devising better heuristics to determine the best order of term variables for assignment is an evident future work. The generator often takes very long to produce its first valid match even for specifications that are not that complex. This issue happens because most of the verifications rely on reasoning, and thus, require time-consuming algorithms.

Moreover, at the moment, not every OWL 2 axiom type can be used to filter domains, while others are only employed in some situations. Another obvious point that deserves more work is the term declaration system that only allows two levels (primitive term variables and composite ones that may only use primitives). In addition to axioms, extending the constructors available to generate composites is also essential to ensure that we can capture more patterns with our specification.

Another limitation is that the generator can work with inconsistent ontologies in a very restricted way, limiting its application in specific scenarios which already have inconsistent bases.

\subsection{Measurements}

One of the fundamental parts to design an empirical framework is to decide what will be measured and how. The second research question (see Section 3.3) that guides this work mentions three quantities (in the physical sense): running time, computational memory and reasoner calls. These concepts, however, need precise definitions and adequate measurement tools.

This section clarifies which quantities will be measured, discussing the most prominent options and justifying the choices made in the experimental design. It also presents the tools used to measure computational time and memory consumed.

\subsubsection{Running Time}

The most problematic of the three quantities considered is running time. First, there is the choice of which type of time to consider: user time or CPU time. The former, also known as "elapsed time" or "wall-clock time" is the time elapsed according to an external reference (e.g. a wall-clock). The later, CPU time refers the time a process spends on the CPU, meaning that when that process is moved out of the CPU (e.g. due to I/O interruptions), no time is accounted for it, but if the process uses more than one core, each of the cores adds to the total CPU time of the process.

The main argument in favour of user time it does not depend on implementation. That is, as long as the timestamps of beginning and end are recorded at the same points of execution. Thus, any tool that marks instants in time and calculates the difference should yield the same result. The problem with this approach is sensitivity to a range of factors. For instance, if the process is interrupted by a higher priority task from the operating system, or if the process is blocked due to $\mathrm{I} / \mathrm{O}$ interruptions.

While CPU time is more robust that user time, its actual meaning varies with hardware, operating system and even actual tools used for measurement. As an example, there is the routine System.nanoTime () avaliable in Java 8's standard library. Its documentation warns: "The value returned represents nanoseconds since some fixed but arbitrary origin time [...]"2. Note that, as the reference is always the same, the time intervals are still meaningful for comparisons.

Matentzoglu [Mat16] discusses both types of time measurements and remarks that more research is needed to determine which one the most informative in ontology-related research.

\footnotetext{
${ }^{2}$ https://docs.oracle.com/javase/8/docs/api/java/lang/System.html\#nanoTime--
} 
In addition to the question of CPU versus user time, the selection of "chronometer" is also important. One method of measuring time is System. nanoTime (), mentioned earlier. Java 8's standard library also includes its counterpart for wall-clock time: System.getCurrentMillis(). However, the System.nanoTime() is recommended over System.getCurrentMilis() by a number of sources [Mat16]. Moreover, other empirical studies in Belief Change and Ontology Repair literature favour System.nanoTime().

In addition to the question of CPU versus user time, the selection of "chronometer" is also important. One method of measuring time is "System.nanoTime()" mentioned earlier. Java 8's standard library also includes its counterpart for wall-clock time: System.getCurrentMillis(). However, the System.nanoTime() is recommended over System.getCurrentMillis() by a number of sources [Mat16].

Instead of relying on these tools, there are external means to evaluate the duration of a program's execution. These methods avoid any changes to the implementation, but also add some overhead in the total running time (though most serious tools account for that and only measure time spent by the program under test).

In this sense, the library Java Microbenchmark Harness, or simply JMH, is a notable option. This library encapsulates a range of instruments to facilitate the development and execution of benchmarks in Java, while avoiding common pitfalls, such as turning off certain features in the Java compiler and in the Java Virtual Machine (JVM) (for instance, dead code elimination, constant folding and loop optimisations). Moreover, it also provides an easy way to change the number of warm-up iterations, time limits per execution, and other execution parameters for the benchmark. It also summarises statistics of execution time of multiple (even forked) runs.

In this sense, the library Java Microbenchmark Harness ${ }^{3}$, or simply JMH, is a notable option. This library encapsulates a range of instruments to facilitate the development and execution of benchmarks in Java, while avoiding common pitfalls, such as turning off certain features in the Java compiler and the Java Virtual Machine (JVM) (for instance, dead code elimination, constant folding and loop optimisations). Moreover, it also provides an easy way to change the number of warm-up iterations, time limits per execution, and other execution parameters for the benchmark. It also summarises statistics of the execution time of multiple (even forked) runs. Its strength lies in the ability to make different measures from different sources via an API that is exposed to the user. In this way, the benchmark designer can select the ideal mechanisms and enjoy the facilities this framework provides.

Other tools can be available depending on the operating system and programs installed. For operating systems with the Linux kernel, one of such tools is perf ${ }^{4}$, which is able to access information from hardware counters to provide statistics of a program's execution. Although the information is somewhat hardware dependent, it is usually able to report CPU time, migrations from CPU cores, wall-clock time, time spent on system calls and other useful information. Further, in most Linux systems it can be integrated directly into JMH's framework.

Due to the nature of the tests this study performs, this thesis favours CPU time over user time. This descision is because the research question Q2 involves a comparision and not the time a user would wait for the result. Each algorithm has its duration measured using perf's task-clock (reported via JMH), which uses arbitrary, but reliable references for time-keeping. Furthermore, $\mathrm{JMH}$ is also employed to avoid common benchmarking issues and caveats.

Unfortunately, the high number of test parameters and methods, summed with the time needed to run a single execution prevent us from using JMH to its full extent. Each test will be performed in "cold runs" (tests where the whole process is run from start to finish), without multiple sequential runs or forks.

This mix of strategies allows us to partially compensate the low number of repetitions per combination of parameters (Section 5.3 elaborates on this issue).

\footnotetext{
${ }^{3}$ https://openjdk.java.net/projects/code-tools/jmh/

${ }^{4}$ https://perf.wiki.kernel.org/index.php/Main_Page
} 


\subsubsection{Memory}

It is also essential to consider how the memory demand changes when modularity is added to the algorithms. In this sense, this study focuses on maximum memory consumed. Keeping track of the maximum is much easier than devising and measuring the average memory consumed during the whole execution, and also introduces less noise. Moreover, measuring average memory consumption requires more sophisticated approaches for which there are few tools.

As for the choice of tools, a possible internal tool is the Java Management interface, via MX Beans ${ }^{5}$. However, in this case, this study favours the information provided by the operating system: the Linux kernel provides a file ${ }^{6}$ with the statistics for each process, it includes, for instance, a metric called virtual memory high watermark. This metric, (abbreviated to VmHWM) reports the peak of resident memory used by a process in all its duration. Recording this value after the process finishes, makes sure that all kinds of overhead are taken into account. Moreover, as JMH will run the program with the methods under test in a separate process (it creates a forked JVM), the benchmarking library will not interfere in the values reported.

The empirical evaluations in Ontology Repair (as similar studies in Belief Change) usually focus on time and do not measure memory. This omission happens even when they mention allocation, garbage collection and other related factors [SQJH08, BS08, Moo10, Nor11, Mat16]. Although there are exceptions [Cób14], there are few studies that establish a careful methodology to measure memory in our setting accurately. Evaluating the best strategy for measuring memory consumption in Ontology Repair applications in Java (and other languages) would benefit the community as a whole, allow researches to make better methodological decisions in empirical evaluations. This task is, however, outside the scope of the thesis and, thus, we will follow the strategy as mentioned earlier, taking into consideration its limitations when discussing the results.

\subsubsection{Reasoner Calls}

The last quantity of interest in this thesis consists in the number of calls made to the reasoner. This measurement is very stable when compared to the other two since it is not hardware-dependent, nor it can be affected by (the usual) external factors. In fact, given a fixed order of iteration of the axioms, the same method would yield the same number of reasoner calls for the same input.

Most of the processing time is typically spent on reasoning, thus reducing the number of reasoner calls is an obvious way to optimise the operations as a whole. In this direction, there is a need to evaluate how the reduction in search space with modules reflects on the number of reasoner calls. That is, to discover the relation, if any, between the number of axioms ignored and the number of reasoner calls performed.

Furthermore, as mentioned in Section 3.3, the effects may vary considerably for MinImps and MaxNons approach, in particular, the results might show that without modularity one of them is better than the other in terms of reasoner calls, but the opposite happen with modularity. The evaluation of this metric will also serve, in conjunction with the other two quantities, to pinpoint causes for performance differences among the approaches and for the impact of modularity.

\subsection{Benchmark Considerations}

To address the second research question (Q2) the implementation of the algorithms, with and without modularity, must be compared empirically. Even though this work does not propose to produce a complete account of the impact of modules on Ontology Repair, we need to guarantee the reliability of the results within the scope established. In particular, the research question inquires about running time and computational memory spent by the modularised versions of the HST-based MinImps and MaxNons computation, when compared to their traditional counterparts. All that for general, existing, OWL 2 DL ontologies.

\footnotetext{
${ }^{5}$ https://docs.oracle.com/javase/8/docs/api/javax/management/MXBean.html

${ }^{6}$ http://man7.org/linux/man-pages/man5/proc.5.html
} 
As commented briefly in Subsections 5.2.1 and 5.2.2, a critical issue with measuring parameters such as time and memory is that both, mostly the former, are sensitive to changes outside the program. Even if one considers a mono-user operating system, whose sole task is to run a single user task at a time, there is still noise that can affect the measurements. Hardware interruptions and high-priority system tasks could influence time measurements (even more if the experiment considers real elapsed time).

Usually, this shortcoming is mitigated by repeating the same iteration many times, interleaved among other ones in a single batch. In this way, by taking a measure of central tendency (e.g. mean) and evaluating the dispersion, the extents of such effects can be determined, and the result still provides useful insights.

The problem in reasoning-dependent algorithms is that they often take too long in a single execution to allow multiple repetitions, and thus mitigate the effect of external factors in the measurements. Often, due to the time that it takes to run these experiments, the authors that are benchmarking different approaches have to limit drastically one parameter, such as the number of ontologies [SQJH08, Moo10], or consider only instances up to a size [CW15].

Another method consists in reducing the number of repetitions and control, as much as possible, the external noise by configuring the environment adequately [Mat16]. This is the option adopted in this thesis. The research question Q2 requires a large number of ontologies, in addition to variations in test type, reasoner and method under test. All these factors, in addition to the execution time of the algorithms, show that it is not feasible to repeat each combination more than a few times.

Next, Subsection 5.3.1 details the actual environments where the experiments discussed in Chapter 6 will run. It also outlines the means used to mitigate influence of external factors on when measuring computational time and memory.

\subsubsection{Experimental Environment}

The experiments to answer the second research question will be performed in two machines whose configuration is summarised in Table $5.1^{7}$.

The machines 1 (M1) and 2 (M2) differ considerably. Both machines will run the main experiment, comparing the approaches for MinImps and MaxNons. What changes between the experiments in each of them is the number of inputs and repetitions per combination of parameters.

In the first machine, only a single process will be run each time. Each execution needs two physical processors, one for the JMH, perf and auxiliary programs (e.g. nice, cgexec), the other for the experiment itself. The other two cores are free to run kernel tasks and other needed applications.

In the second, as it has more of cores than the first, the experiment performs ten executions simultaneously. This parallelism requires additional effort to guarantee that each process does not interfere with the others significantly. Also, the analysis needs to be more cautious when evaluating the computational resources consumed.

Next, this subsection describes briefly some settings enforced on the operating system and JMH as a means to control external interference on the methods being compared. Unless explicitly stated, the action was taken on both machines.

Most system interruptions redirect from the cores running the experiments to a set of "free" cores. Next, security and monitoring components were disabled. Only essential services of the operating system were left running, in addition to a SSH server to allow monitoring and retrieval of the results.

Technologies of frequency scaling (either for saving energy or improving performance in short bursts) were turned off. A fixed frequency is set differently for each machine, on M1 the CPUs were capped at $3.6 \mathrm{GHz}$, fast enough for our experiments while preventing issues due to power and temperature variations. On M2, thanks to the controlled environment, the frequency was set the maximum baseline of $2.0 \mathrm{GHz}$.

\footnotetext{
${ }^{7}$ M1's hardware correponds to an Acer Aspire GX 783-BR13 and M2 to a Hetzner AX 160, with an extra 32GB of RAM
} 


\begin{tabular}{lll}
\hline Component & Machine M1 & Machine M2 \\
\hline CPU & & \\
Model & Intel i7-7700 & AMD EPYC 7401P \\
Physical cores & 4 & 24 \\
Baseline frequency & $4.0 \mathrm{GHz}$ & $2.0 \mathrm{GHz}$ \\
Memory & & \\
Type & DDR 4 & DDR 4 \\
Capacity & $16 \mathrm{~GB}$ & $160 \mathrm{~GB}$ \\
Frequency & $2400 \mathrm{MT} / \mathrm{s}$ & $2400 \mathrm{MT} / \mathrm{s}$ \\
Access type & $\mathrm{SMP}$ & $\mathrm{NUMA}$ \\
Storage & & \\
Type & $\mathrm{HDD}$ & $\mathrm{NVMe}$ \\
Capacity & $1 \mathrm{~TB}$ & $1920 \mathrm{~GB}$ \\
Operating system & & \\
Kernel version & 4.19 & 3.10 \\
Distribution & Debian 10 & Cent OS 7.6 \\
JRE version & 1.11 & 1.8 \\
Java options & & \\
Initial Memory Pool (-Xms) & $2 \mathrm{~GB}$ & $2 \mathrm{~GB}$ \\
Maximum Memory Pool (-Xmx) & $12 \mathrm{~GB}$ & $12 \mathrm{~GB}$ \\
\hline
\end{tabular}

Table 5.1: Experimental machines' specification

Additionally, for each physical core, only one virtual core was left enabled. This device increases the stability by reducing competition for CPU by different tasks and avoiding CPU migration (shifts in which core is processing a program).

Machine 2 also requires other preventive actions due to the parallelism in the benchmark execution. For instance, the maximum amount memory each process can use is limited to $14 \mathrm{~GB}$, to prevent a process from allocating all available memory and forcing the others to wait. With this capacity, there is still enough memory left for the OS and other programs. Further, it also explicitly reduce the likelihood that the memory pages of the process in execution will be swapped to disk.

While in M1 the memory access architecture is symmetrical, in M2 the memory access is not uniform (hence, NUMA - non-uniform memory access). Therefore, in M2 script determines for each CPU core the memory nodes that it can access (the closest ones). The configuration made prevent some cores from having slower memory access than others.

In both machines, when running the benchmarks we use only two forks of JVM, one for JMH and the other for the experiments. The tests are run in cold mode, meaning that the whole process is shut-down at the end of the execution. The runs are generated, randomised (order of execution) and the split among the available cores. Hence, each core runs an independent subset of all iterations.

Before dispatching the processes to each core, the system caches are cleaned. Then each core executes 40 warm-up runs. After that, the accounted runs follows until each process terminates its batch. While there is at least one process executing its effective payload, the others keep running a random instances. All the processes stop once everyone has concluded its assigned (effective, accounted for) iterations. These warm-up and tail executions contribute to keep the measurements uniform regardless of the order and core they are processed.

Finally, the experiments' processes are run with administrator privileges, with a high priority (low niceness value), so that they can stay in the CPU as much as possible without being interrupted by other tasks. 


\subsection{Other Experimental Parameters}

This section details the additional choices undertaken regarding experiments. In particular, Subsection 5.4.1 discusses the dataset used to feed the corpus generator (from Section 5.1) while Subsection 5.4.2 introduces the OWL reasoners employed.

\subsubsection{Dataset Choice}

Section 5.1 introduced the tool developed to create a reproducible corpus for the experiments. The generator needs two datasets: a corpus of ontologies and a set of test case templates (these in the format discussed in Subsection 5.1.1). This subsection exposes the arguments supporting the choices of ontology corpus ontologies and the test templates devised.

One of the most popular sources of ontologies for testing is the BioPortal ${ }^{8}$ repository [Hor11, CW15, Mat16]. Among other curation services, it provides a corpus of ontologies, most of them from the biomedical domain, many of which are used to build semantic applications in their respective fields. BioPortal also implements a convenient API to download its ontologies, facilitating the construction of a corpus.

Even with these resources, often, these ontologies need to be filtered and preprocessed [Hor11, MBP13b, Mat16]. Instead of devising its own preprocessing methods, this thesis employs a carefully prepared snapshot of the BioPortal ${ }^{9}$, produced by Matentzoglu and Parsia [MP17]. The snapshot provided already solves issues regarding ontologies imported by URLs and fixes some minor OWL 2 profile violations. Moreover, it includes metadata that can be used to understand the overall characteristics of the corpus (for instance, it catalogues the number of axioms and profile of each ontology).

Previous studies in Ontology Repair literature inspired the choice of test case templates. Due to the practical limitations, only two templates were applied. The first corresponds to a contraction situation: the generator identifies an implicit subclass relation (not involving $\top$ or $\perp$ ) and requires its removal. Subclass relations are one of the most common types of entailments used for testing reasoning-based applications in Ontology Repair [Hor11, CW15]. Also, they are available even in the OWL 2 EL, the least complex profile (in terms of computational complexity). For conciseness, this template will be referred as case type T1.

The second test template represents one crucial issue with ontologies: inconsistency. Few of the ontologies in the dataset are inconsistent. Moreover, they could be inconsistent due to very different reasons. Also, technical factors limit the ability of the corpus generator to operate with inconsistent ontologies (as discussed in Section X). Hence, this test specification mimics a technique employed by Bonatti et al. [BFPS15]. This method introduces an inconsistency in a controlled way. The picks two concepts $\mathrm{C}$ and $\mathrm{D}$ such that no subclass relation between them is entailed. Then, it selects a superclass of each, respectively $\mathrm{C}_{1}$ and $\mathrm{D} 1$, such that they are not subclass nor superclass of each other. The specification then adds the axiom $\mathrm{C}_{1} \sqcap \mathrm{D} 1 \sqsubseteq \perp$ to the output ontology via the action block. Meanwhile, a catalyst requires the addition of $\mathrm{C} \sqcap \mathrm{D}(a)$, simulating the case of a revision that may cause inconsistency. For conciseness, this template will be referred as case type T2.

The corpus generation and the experiments themselves are computationally intensive tasks. Hence, only three instances of each combination of ontology and test template were attempted to be built. All three instances of an ontology-template pair should be given within one hour in the environment M1 (see Table 5.1).

The generation produced a total of 1664 benchmarking inputs. JFact ${ }^{10}$ was the reasoner used for generation. It was easily integrated with the generation framework via the OWL API (version 5.1.11). Hermit was another candidate, but its performance was much worse on the enriched ontology that OWL2DL- CCC uses to handle complex (but asserted) concepts. The enrichment consists of

\footnotetext{
${ }^{8}$ https://bioportal.bioontology.org/

${ }^{9}$ https://zenodo.org/record/439510

${ }^{10} \mathrm{http}: / /$ jfact.sourceforge.net/
} 
introducing new concept names via equivalent classes axioms so that they represent a complex concept in the original ontology.

As future work, the experiments can be extended number and complexity of test templates. In particular, including a test template involving incoherence, another remarkable faulty behaviour in most cases that is as DL agnostic as inconsistency. The corpus also can be extended using ontologies from other domains and allowing a longer timeout and a higher memory limit.

\subsubsection{OWL Reasoner Choice}

The main criteria on this decision were: compatibility with the OWL API version 5 and the ability to handle OWL 2 DL ontologies. The reason why OWL API 5 was chosen even though fewer reasoners support is due to bugs on the module extraction and atomic decomposition components which were only corrected recently ${ }^{11}$.

Other notable reasoners were excluded for other reasons. For instance, ELK ${ }^{12}$ only handles OWL 2 EL ontologies and Konclude ${ }^{13}$ has a very complicated routine to integrate with the OWL API, and its interface does not allow general entailment queries. After this initial filtering, three reasoners remained: Hermit ${ }^{14}$, Fact $++{ }^{15}$ and JFact. Due to some issues when building Fact ++ with OWL API $5^{16}$, only the other two remained. More specifically the experiments discussed here and in Chapter 6 use Hermit version 1.4.3.517 and JFact version 5.0.3, both via OWL API version 5.1.12. The same versions of JFact and OWL API were employed to produce the benchmark corpus discussed in Subsection 5.4.1.

Another factor that limits the number of reasoners is the time required to perform the experiments. Even with only two reasoners and two test templates and few repetitions per combination of parameters, the whole experiment takes weeks both in M1 and well as in M2.

The results obtained regarding the number of reasoner calls can still be generalised regardless of the reasoner. Nevertheless, as future work, to obtain more reliable conclusions, the experiment should be repeated with a broader range of reasoners.

\subsection{Supporting Material}

Additional supporting material, including the source code for the experiments, data sets and set-up scripts can be found at https://www.ime.usp.br/ ricardof/thesis material.

Moreover, the implementations of Belief Base Change operations for OWL 2 DL ontologies is available as a source code repository: https://gitlab.com/rfguimaraes/owl-change/-/tags/0.0. 4-alpha2.

The code for OWL2DL-CCC is currently hosted at https://gitlab.com/rfguimaraes/owl2dl-ccc. The soruce code repository for OWL2DL-Stratification, the stratification library, can be found at https://gitlab.com/rfguimaraes/owl2dlstratification.

\footnotetext{
${ }^{11}$ see https://github.com/owlcs/owlapi/issues/704 for an example

${ }^{12}$ https://www.cs.ox.ac.uk/isg/tools/ELK/

${ }^{13}$ https://www.derivo.de/en/products/konclude/

${ }^{14} \mathrm{http}: / /$ www.hermit-reasoner.com/

${ }^{15}$ http://owl.cs.manchester.ac.uk/tools/fact/

${ }^{16}$ https://bitbucket.org/dtsarkov/factplusplus/issues/81/error-with-owl-api-510-and-5110
} 


\section{Chapter 6}

\section{Empirical Evaluation}

This chapter details the experiments tailored to answer the research question Q2 presented in Section 3.3. In short, these experiments produce data to allow comparison of five different methods to compute Belief Change and Ontology Repair constructions. Further, this chapter presents and discusses the results obtained, highlighting the most relevant points to the research question at hand.

Due to pragmatical considerations, instead of a single experiment, this thesis considers three. Section 6.1 explains the motives of this separation and describes each sub-experiment.

\subsection{Experiments}

Next, this section lists the three experiments considered in this chapter:

Full-M1 : Runs the methods that compute the whole MinImps and MaxNons in the machine M1 (Table 5.1).

Full-M2 : Runs the methods that compute the whole MinImps and MaxNons in the machine M2 (Table 5.1).

Single-M2 : Runs the methods that compute a single MaxNon or a cut of all MinImps (a single closed path in the HST). Only run in machine M2.

The experiment Full-M1 and Full-M2 serve the same purpose and compare the same methods, but as the environment differ, the parameters and controlling devices in each case also change. In machine M1, only a single process is run at a time, while ten are run concurrently in M2 (Subsection 5.3.1 discusses the measures were taken to reduce the chances that one process interferes with another). Additionally, Full-M1 uses only 1112 out of the 1664 inputs generated (it removes the third pair for each ontology-template combination, if there was one). Even so, the results on each machine should be the same when comparing methods. Otherwise, a more in-depth analysis must be conducted to identify which factors were responsible by the variation.

As for Single-M2, it computes the minimal result need to obtain a Belief Change operation. The methods studies compute a single MaxNon or a cut of all MinImps. The function stopEarly implements this behaviour, forcing Algorithm 1 to stop before the whole sets are computed. In the case of MaxNons, the algorithm stops when the first node is calculated, the result is a maximal repair. For MinImps, stopEarly makes the algorithm stop at the first closed path; it may be the case that the cut is not $\subseteq$-minimal (thus, the outcome would not be optimal). Still, given any cut and the nodes that it intersects, a minimal one can be determined easily. This experiment limits its input to the same 1112 instances of Full-M1.

Moreover, since each execution in Single-M2 requires fewer operations (in general), another parameter was also included: whether Algorithm 1 follows a breadth-first search (using a queue) or 


\begin{tabular}{llll}
\hline Parameter & Full-M1 & Full-M2 & Single-M2 \\
\hline Environment & M1 & M2 & M2 \\
Total number of input files & 1112 & 1664 & 1112 \\
Number of reasoners & 2 & 2 & 2 \\
Number of methods & 5 & 5 & 10 \\
Repetitions per combination & 3 & 5 & 6 \\
Timeout (seconds) & 300 & 480 & 420 \\
Total number of runs & 33360 & 83200 & 133440 \\
\hline
\end{tabular}

Table 6.1: Parameters of each sub-experiment for research question Q2

a depth-first search (using a stack). The choice of implementation should not affect the MaxNonsbased methods, as they only compute the root node. Yet, both implementations can differ considerably in the computational resources spent for MinImps-based ones.

For all three experiments the execution procedure was the same:

1. Prepare the environment (see Section 5.3).

2. Upload the programs and data files.

3. Randomise the executions.

4. Split the number of executions by the number of processes to be run.

5. Launch all processes, each with its subset of warm-up, effective and tail (padding) executions.

6. Wait for all processes to conclude.

7. Collect and parse the result and log files.

In contrast to the uniformity of procedure, the actual parameters of each experiment differs, as summarised in Table 6.1.

The methods compared in Full-M1 and Full-M2 are listed below. They use the algorithms described in Subsection 2.3.4 and Chapter 4.

Basic-MI : Computes the MinImps without modularity. Uses the syntactic connectivity enlargement heuristic (Algorithm 7) and divide and conquer shrinking (Algorithm 8).

SME-MI : Computes the MinImps doing a single module extraction (before calling Algorithm 1). Uses trivial enlargement (Algorithm 6) and divide and conquer shrinking.

MU-MI : Computes the MinImps using Algorithm 1, that is, updating the module during the computation. Utilises the same heuristics as SME-MI.

Basic-MN : Computer the MaxNons without modularity. It computes each MaxNon with syntactic connectivity shrinking (Algorithm 10) and iterative enlargement (Algorithm 11).

SME-MI : Computes the MaxNons doing a single module extraction (before calling Algorithm 1). Uses Algorithm 9 and iterative enlargement (Algorithm 11).

For Single-M2, the same fundamental methods are considered. However, they are accompanied by a suffix (Q) or (S) to indicate whether they use queue or stack implementations, respectively. For instance, SME-MI(Q) denotes the method that computes a single cut of all MinImps using queue as implementation (i.e. it navigates using a breadth-first order). Due to the time required for Full-M1 and Full-M2 to finish, the structure backing the HST in these experiments was always a queue. 
When comparing the methods there are three quantities of interest: the number of reasoner calls, computational time and memory. The number of reasoner calls is easily measured and does not vary across executions with the same parameters; time and memory are different. CPU time is measured with perf's task clock measure (reported via JMH). For memory, only the high watermark of virtual memory is considered (VmHWM). Section 5.2 discusses these quantities and associated tools in more detail.

Section 6.2 displays the results of these experiments. Further, it justifies the choice of metrics and methods of presentation used to effectively compare the methods.

\subsection{Results}

The main purpose of this sectionis to discuss the results obtained from the experiments mentioned in Section 6.1. Before that, it is necessary to explain the main comparison metrics chosen.

First, the most important is the base 2 logarithm of the fold change between two values $(\log 2 \mathrm{FC})$. The fold change between two measurements $m_{1}$ and $m_{2}$ is the ratio $\frac{m_{1}}{m_{2}}$. This metric indicates by how much $m_{1}$ changes in terms of $m_{2}$. For instance, if $m_{1}=4$ and $m_{2}=2$, then $F C\left(m_{1}, m_{2}\right)=2$, if $m_{1}=m_{2}, F C\left(m_{1}, m_{2}\right)=2$. One problem with this metric is that it distorts comparison when $m_{1}<m_{2}$ since $F C\left(m_{1}, m_{2}\right)$ is restricted to the interval $(0,1)$. Matentzoglu [Mat16] circumvents this problem by taking $-F C\left(m_{2}, m_{1}\right)$ instead of $F C\left(m_{1}, m_{2}\right)$ whenever, $m_{1}<m_{2}$. Instead, the evaluation here adopts a more common approach by taking the logarithm base 2 of the actual fold change. In this way, when the $m_{1}=m_{2}, \log 2 F C\left(m_{1}, m 2\right)=0$, indicating no change, when $m_{1}$ is the double of $m_{2}: \log 2 F C\left(m_{1}, m_{2}\right)=1$ and if its the other way around $\log 2 F C\left(m_{1}, m_{2}\right)=-1$. Thus, $\log 2 F C$ provides a straightforward method to understand the variations of the quantities measured across the methods evaluated.

The fold changes computed compare measurements of the same quantity in runs that differ only regarding the method. Instead of pairing repetitions of each run, the fold changes are calculated over means of the repetitions of executions. For instance, in experiment Full-M2, it can be computed the average of reasoner calls for each triple of input, reasoner and method. Then, selecting only two methods, each pair of input and reasoner will have an average number of checker calls for each method. The $\log 2 \mathrm{FC}$ is then calculated over the averaged values. Hence, each pairing of means produces a value of $\log 2 \mathrm{FC}$ that will feed the analysis.

One issue with the mentioned approach is that by taking the mean alone, the information of dispersion per group is lost. This information is vital to attest that the stability of the experiments. Hence, the variation in each group of executions is also quantified using the coefficient of variation $(\mathrm{Cov})$. The coefficient of variation is the quotient of the standard deviation divided by the mean. The distribution of this metric indicates the robustness of the results. Table 6.2 summarises statistics of the coefficient of variation for all experiments and quantities considered, facilitating the presentation of the remaining results.

Although some values of coefficient of variation are large (above 50\%), they are rare. In all cases, the third quartile for the coefficient of variation is below $7 \%$ and the average below $5 \%$. Hence, the results presented later will still be significant, even ignoring the dispersion when analysing the $\log 2 \mathrm{FC}$ distribution.

The last noteworthy metric in this chapter is a correlation coefficient. This section also displays the correlation between $\log 2 \mathrm{FC}$ and other parameters to aid in the explanation of the results, in particular, to identify which factors make modularity more or less effective. Among the existing correlation metrics this study focuses on Kendall's $\tau$ [Ken38]. This metric ranges from -1 to 1 . Positive values indicate a direct monotonic association; negative ones inverse monotonic association. Values closer to 0 indicate a weaker association, while closer to either 1 or -1 indicate stronger ones. As opposed to Pearson's coefficient, it is a non-parametric coefficient, and that can measure association even when the relation is not linear. Also, it is generally more robust than its counterpart Spearman's $\rho[C D 10]$.

For the most notable comparisons, the distribution of the $(\log 2)$ fold change will be displayed 


\begin{tabular}{lclccccccc}
\hline Experiment & \multirow{2}{*}{$\mathrm{N}$} & \multicolumn{1}{c}{ Quantity } & Mean & Std & Min & $25 \%$ & $50 \%$ & $75 \%$ & Max \\
\hline Full-M1 & \multirow{2}{*}{9770} & Reasoner calls & $0.12 \%$ & $0.79 \%$ & $0.00 \%$ & $0.00 \%$ & $0.00 \%$ & $0.00 \%$ & $36.03 \%$ \\
& & Processing time & $1.80 \%$ & $1.80 \%$ & $0.01 \%$ & $0.67 \%$ & $1.24 \%$ & $2.23 \%$ & $16.35 \%$ \\
& & Memory & $3.38 \%$ & $6.20 \%$ & $0.01 \%$ & $0.52 \%$ & $1.55 \%$ & $3.40 \%$ & $65.01 \%$ \\
Full-M2 & \multirow{2}{*}{14515} & Reasoner calls & $0.12 \%$ & $0.67 \%$ & $0.00 \%$ & $0.00 \%$ & $0.00 \%$ & $0.00 \%$ & $22.65 \%$ \\
& & Processing time & $4.54 \%$ & $2.20 \%$ & $0.17 \%$ & $2.99 \%$ & $4.34 \%$ & $5.72 \%$ & $25.22 \%$ \\
& & Memory & $1.96 \%$ & $1.84 \%$ & $0.00 \%$ & $0.58 \%$ & $1.36 \%$ & $3.01 \%$ & $34.20 \%$ \\
Single-M2 & \multirow{2}{*}{20649} & Reasoner calls & $0.18 \%$ & $1.51 \%$ & $0.00 \%$ & $0.00 \%$ & $0.00 \%$ & $0.00 \%$ & $97.98 \%$ \\
& & Processing time & $4.86 \%$ & $2.18 \%$ & $0.04 \%$ & $3.35 \%$ & $4.67 \%$ & $6.01 \%$ & $27.91 \%$ \\
& & Memory & $3.37 \%$ & $1.78 \%$ & $0.03 \%$ & $1.97 \%$ & $3.45 \%$ & $4.50 \%$ & $32.72 \%$ \\
\hline
\end{tabular}

Table 6.2: Statistics of the coefficient of variation in each combination of parameters, for each experiment and each quantity measured

per pair of methods via raincloud plots $\left[\mathrm{APW}^{+} 19\right]$. These sophisticated graphs combine a boxplot, a scatterplot and an estimation of the probability given the observations (via a kernel density function with smoothing factor 0.2). In some cases, the raincloud plot will be omitted for conciseness. For instance, most of the results between Full-M1 and Full-M2 are very similar, hence instead of presenting the raincloud plot for both, Full-M2 is favoured, and the result for Full-M1 is given textually. Later, this section details how each these plots is built. The data and script utilised to build these plots are available with other supporting material at https://www.ime.usp.br/ ricardof/ thesis_material.

\subsubsection{Preliminary Considerations}

It is also important to guarantee the dataset on the experiments has a certain variability with respect to modularity and concerning computation of MaxNons and MinImps. In Figure 6.1, each point represents an case file. In the majority of the cases, the signature is small the problems sizes are well distributed nonetheless: there is no correlation between ontology size and the size of the input signature.

It is also essential to know how the dataset varies concerning modularity and the number and size of MaxNons and MinImps. In Figure 6.1, each point represents an input (ontology-template pair). This figure shows that, in the majority of the cases, the signature is small, and the problems sizes are well distributed. Also, there is no correlation between ontology size and the size of the input signature.

Figure 6.2 shows that in most cases, the module sizes are small when compared to the original ontology size. Thus we have to take this into account when evaluating the results obtained.

Next, Figures 6.3 and 6.4 display the distributions of MinImps and MaxNons, respectively, in quantity and the average size of elements. Although the sizes of MaxNons are considerably larger than those of MinImps, this expected due to the minimality/maximality duality. Moreover, for both constructions, the cardinality of the sets varies considerably, ; this is a good indication of the dataset's diversity.

Also, Table 6.3 shows that the ontologies considered in the experiments differ in complexity. The ontologies are distributed among different combinations of OWL 2 profiles.

\subsubsection{Construction of Complete Sets}

This subsection discusses the results for Full-M1 and Full-M2, with a particular focus on the second since most the results are similar and the input for later is a superset of the input for the first one. Most of the comparisons between methods are made by pairs (that is one method against the other). Note that this chapter does not compare every pair of methods, only the most relevant 


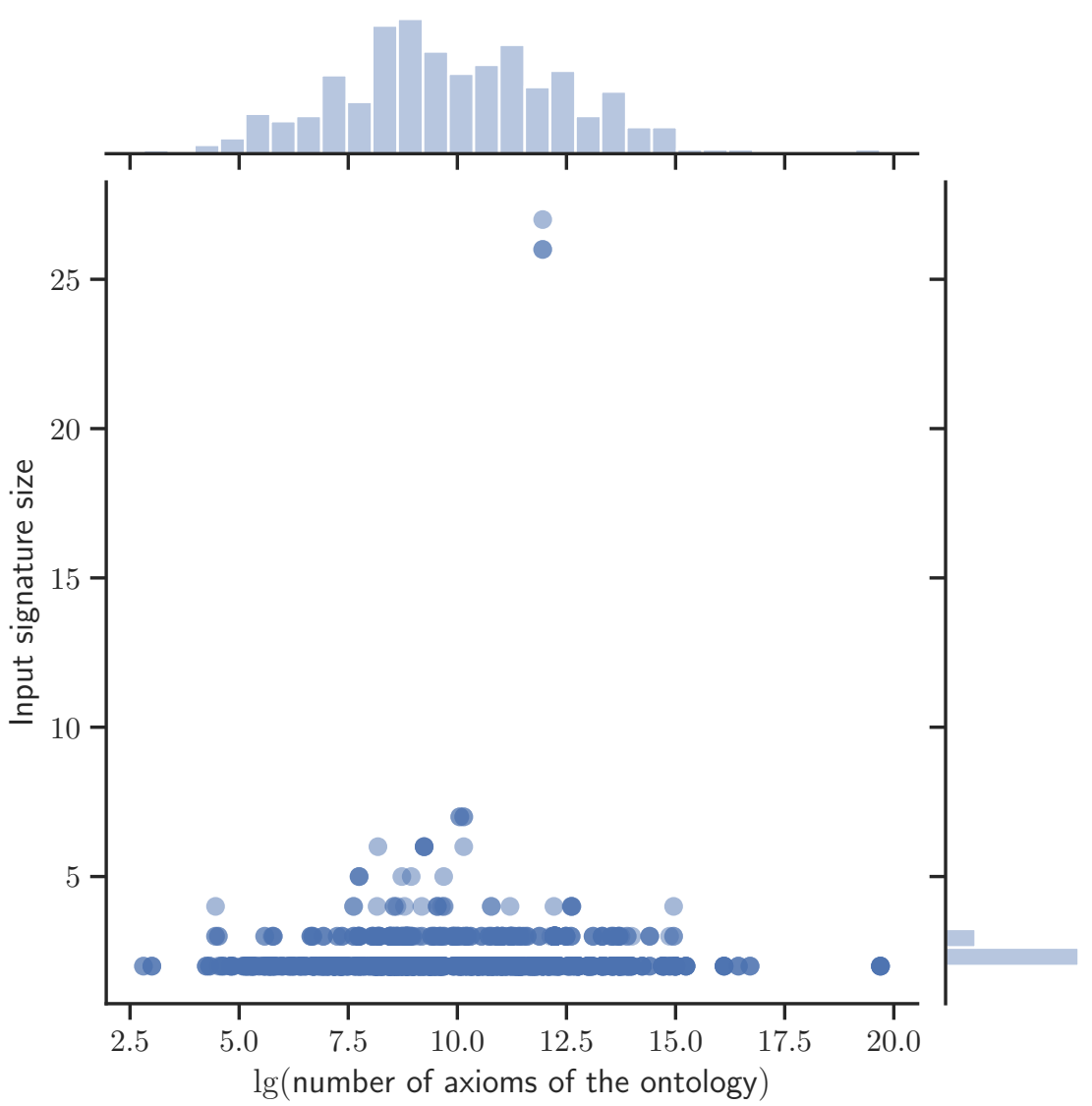

Figure 6.1: Distribution of inputs according to ontology size and input signature size

to the research question Q2.

As discussed in Section 5.2, the computational time is measured using perf's task-clock (which uses a fixed but arbitrary clock) and, which uses hardware counters to account for the time that a process spent in the CPU. In reality, other time measurements were made; they are included in the data that is part of the supporting material (https://www.ime.usp.br/ ricardof/thesis_material).

The finer-grained modularity-based MinImps computation (MU-MI) is ignored for most of this subsection. This omission is because this method offers no significant improvement over the single module extraction (SME-MI). Table 6.4 attests the similarity in of both modularity approaches.

The values in Table 6.4 indicate that there was almost no change in performance when using MU-MI instead of SME-MI, although the positive trend of mean, median and quartiles indicates an improvement. The means and median close to 0 corroborate this fact. So does the symmetry of the first and third quartiles around the median. Further, even these measurements are also close to 0 . While the maximum and minimum values are very distant from the first and third quartiles respectively and but even so, they do not represent drastic changes.

\section{Reasoner Calls}

Figure 6.5 depicts the distribution of the $\log 2 \mathrm{FC}$ comparing MinImps computation with and without modularity in Full-M2. In other words, the raincloud plot indicates relative changes when using SME-MI instead of Basic-MI. Values above 0 indicate that modularity effective in reducing the number of reasoner calls. Since this is the first of many raincloud plots discussed in this chapter, its composition is detailed next as a model to understand the other ones presented.

Let $c$ be a case input (generated using the ontology and test case specification datasets). Without loss of generality suppose that this case is of type T1 (a contraction of an implicit concept inclusion axiom) and that the reasoner used was JFact. Then, in the top left raincloud plot of Figure 6.5, 


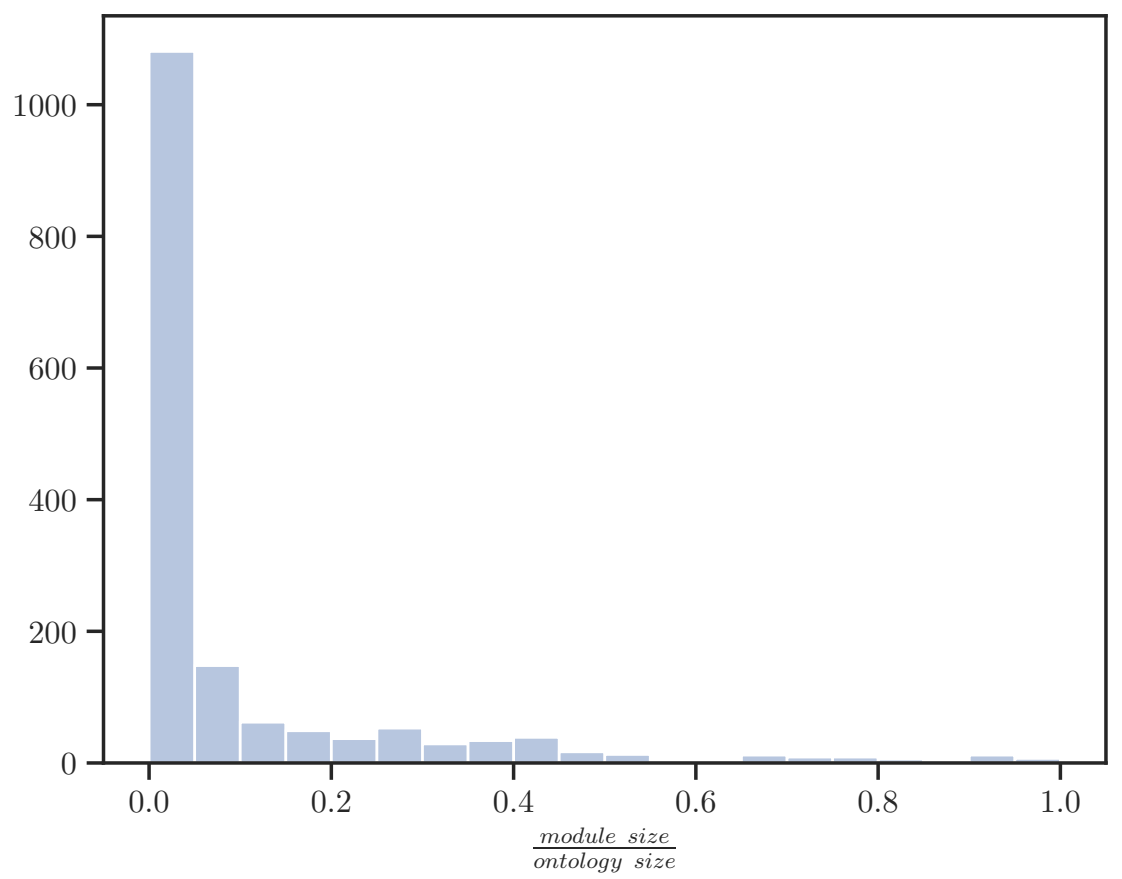

Figure 6.2: Frequency histogram of input cases according to relative module size

\begin{tabular}{lr}
\hline Profile(s) & Ontologies \\
\hline EL & 38 \\
QL & 6 \\
RL & 7 \\
EL + QL & 40 \\
EL + RL & 3 \\
QL + RL & 4 \\
EL + QL + RL & 60 \\
DL & 126 \\
Total & 284 \\
\hline
\end{tabular}

Table 6.3: Number of considered ontologies per OWL 2 profile (note that each category excludes the others, e.g. EL+RL means that the ontology is in $O W L 2 E L$ and $O W L 2 R L$ but not in $O W L 2 Q L)$.

\begin{tabular}{lclccccccc}
\hline Experiment & $\mathrm{N}$ & \multicolumn{1}{c}{ Quantity } & Mean & Std & Min & $25 \%$ & $50 \%$ & $75 \%$ & Max \\
\hline Full-M1 & 2137 & Reasoner calls & 0.0082 & 0.0508 & -0.1701 & 0.0000 & 0.0000 & 0.0000 & 0.8171 \\
& & Processing time & 0.0120 & 0.0696 & -0.3535 & -0.0125 & 0.0038 & 0.0254 & 1.0517 \\
& & Memory & 0.0027 & 0.1450 & -1.2987 & -0.0192 & 0.0018 & 0.0235 & 1.6246 \\
Full-M2 & \multirow{2}{*}{3194} & Reasoner calls & 0.0083 & 0.0469 & -0.1701 & 0.0000 & 0.0000 & 0.0000 & 0.8171 \\
& & Processing time & 0.0099 & 0.0901 & -0.4969 & -0.0362 & 0.0060 & 0.0493 & 1.4773 \\
& & Memory & 0.0103 & 0.1299 & -1.8171 & -0.0122 & 0.0048 & 0.0292 & 1.4075 \\
\hline
\end{tabular}

Table 6.4: Statistics of the log2FC from SME-MI to MU-MI Full-M1 and Full-M2

there is a dot in the scatterplot part (the "rain") which indicates the logarithm base 2 (lg) of the ratio between the average number of reasoner calls spent by Basic-MI for the input $c$ using JFact, repeated five times and the average number of reasoner calls spent by the five repetitions of SME-MI when processing the input $c$ with JFact as reasoner. In symbols, for each input case $c$ of type T1, 


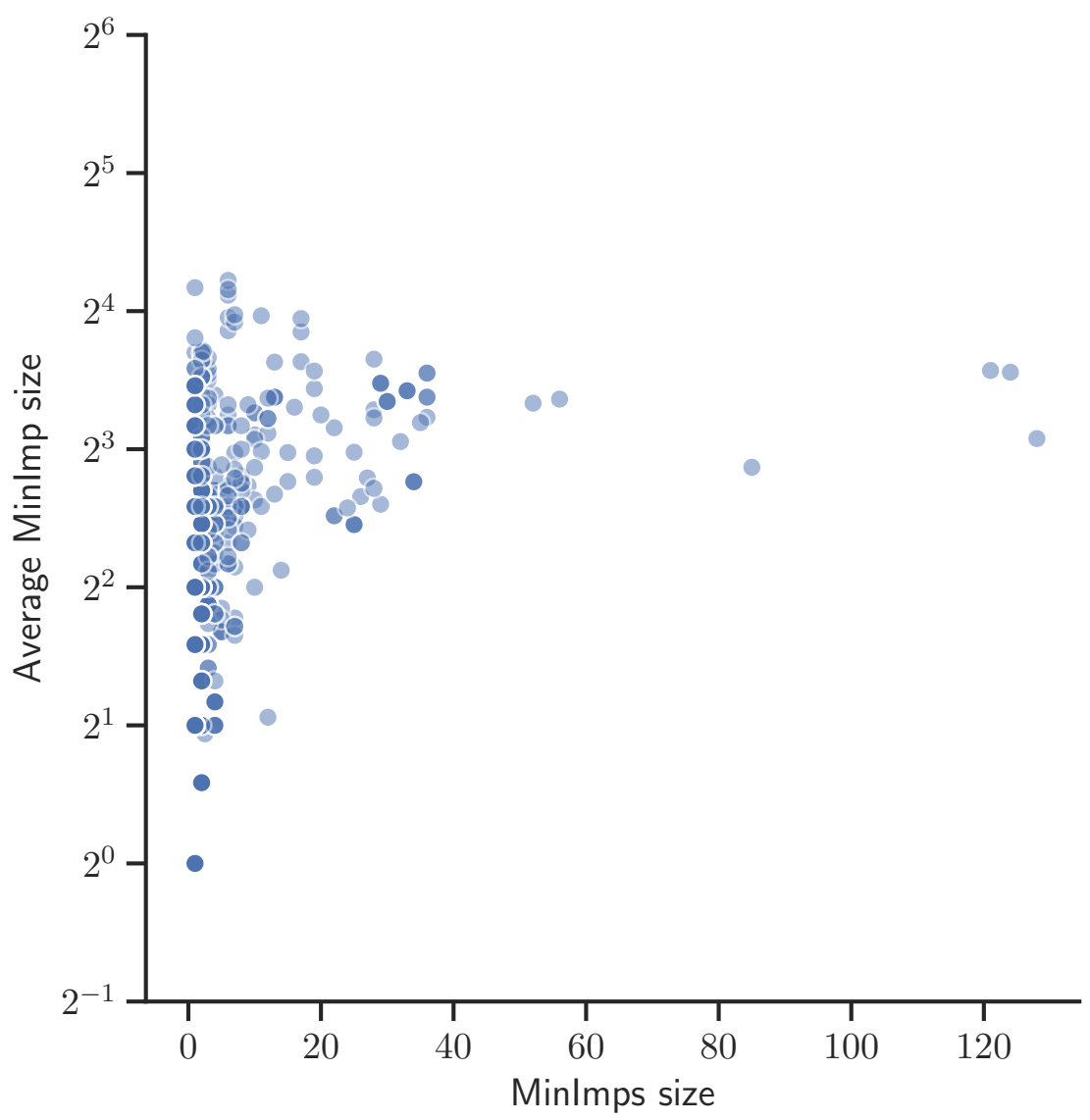

Figure 6.3: Distribution of input according to MinImps size and average number of axioms per MinImp

with reasoner JFact, there will be a dot at $\log 2 \mathrm{FC}$ calculated as:

$\lg \left(\frac{\text { average reasoner calls performed with Basic-MI when processing } c \text { with JFact }}{\text { average reasoner calls performed with SME-MI when processing } c \text { with JFact }}\right)$.

Each figure will have four raincloud plots for the different combinations of reasoners and input types. The dots marking the value of $\log 2 \mathrm{FC}$ for each input compose the scatterplot from which the boxplot and kernel density estimation are derived to indicate the distribution of $\log 2 \mathrm{FC}$ for all inputs considered.

Unsurprisingly, there close to no variation in the fold change when the reasoner changes. In principle, the number of calls to the reasoning mechanism is the same, regardless of the reasoner. In all situations, the number of reasoner calls when computing MinImps was improved with module extraction.

For the use cases of type T1, the reasoner calls log2FC's median and mean are around 4 and 5 , but with a higher estimated probability density with log2FC between 3 and 4 . This means that, in average SME-MI makes only $\frac{1}{16}$ of the calls made by Basic-MI. Further, looking at the raincloud plots in Figure 6.5, values of $\log 2 \mathrm{FC}$ up to 6 are not that unlikely. This fact highlighted by the third quartile in the boxplot, the high estimated probability density until $\log 2 \mathrm{FC} 6$ and the number of observations in the interval between 0 and 6 . As a reminder: a $\log 2 \mathrm{FC}$ of 6 indicates that the modularised approach makes $\frac{1}{2^{6}}=\frac{1}{64}$ of the reasoner calls of the non-modularised one.

As for the second use case type (T2), the improvement was smaller. While the mean is still above 4 in all cases, the probability density distribution is more concentrated at its peak, which is around $\log 2 \mathrm{FC}$ of 3.5. The first quartile and median are lower than before: between 2 and 3 for the first and 3 and 4 for the latter. Moreover, both the estimation of probability density and the 


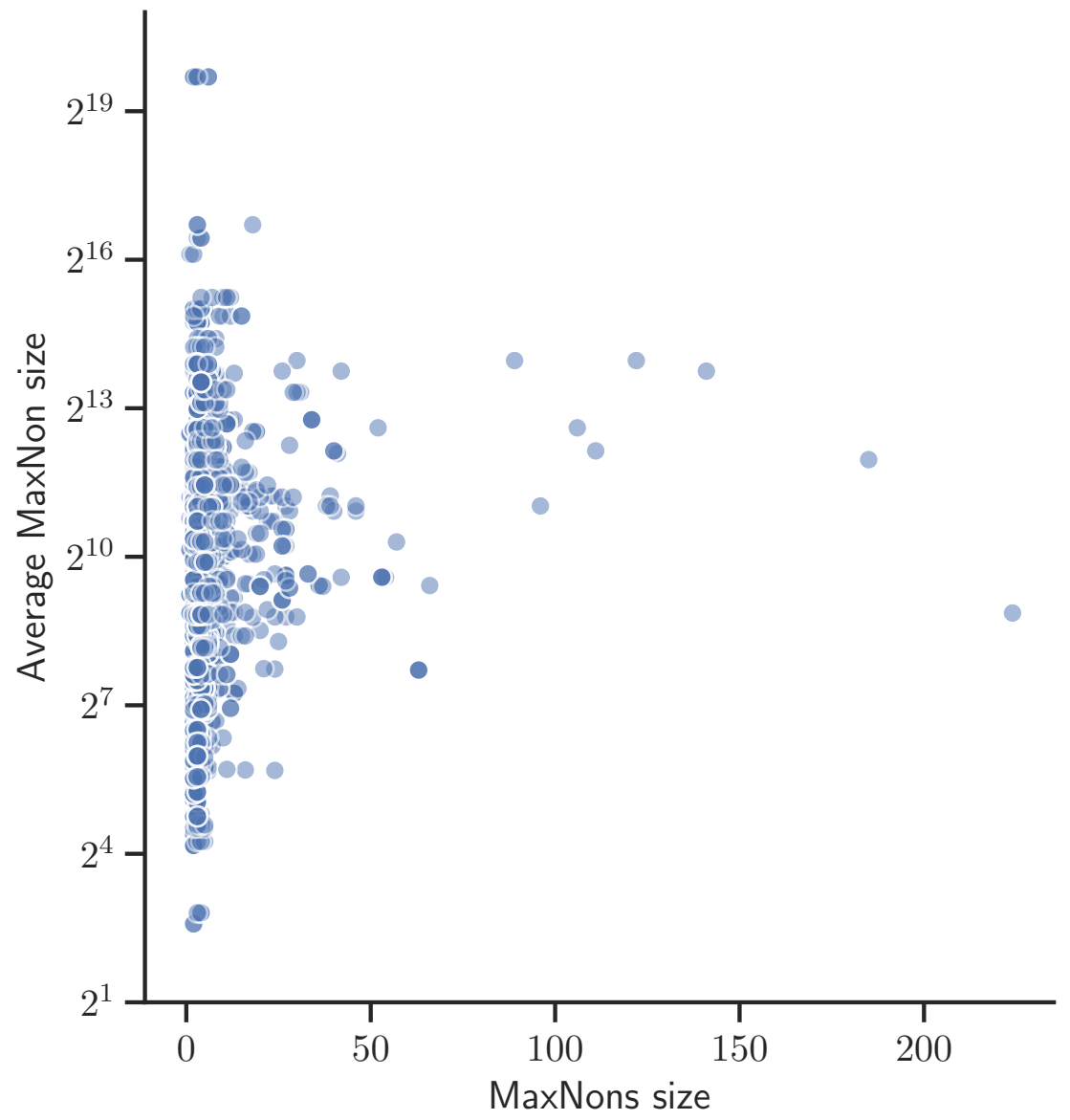

Figure 6.4: Distribution of input according to MaxNons size and average number of axioms per MinImp 

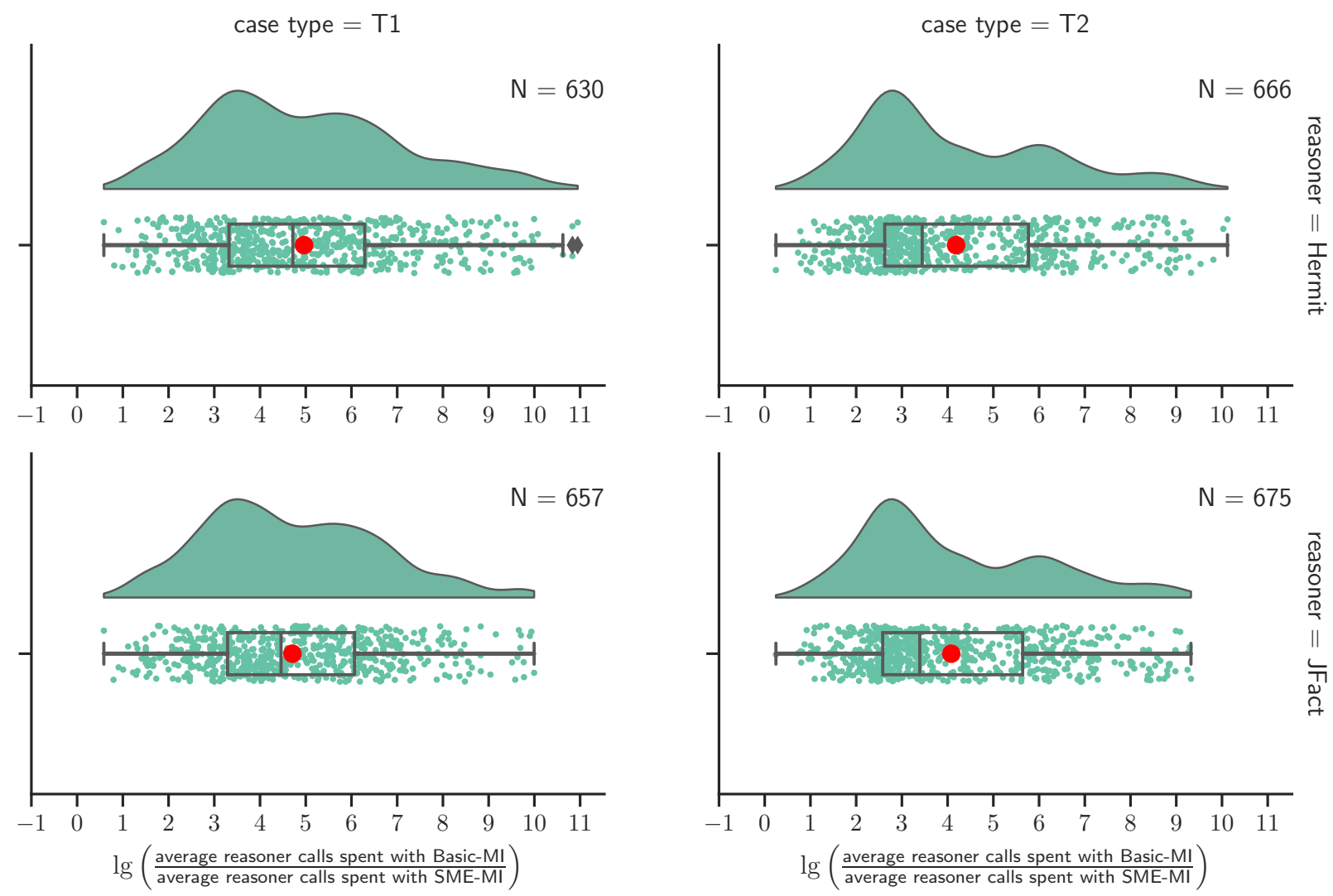

Figure 6.5: Distribution of log2FC for reasoner calls spent in average by Basic-MI and SME-MI in Full-M2

observations plotted indicate fewer occurrences of cases that passed the mean values in each case.

Figure 6.6 displays the impact of modularity on the number of reasoner calls for the MaxNonsbased approach, that is, the change in reasoner calls from using Basic-MN to SME-MN. As with Figure 6.5, there is almost no distinction of $\log 2 \mathrm{FC}$ across the reasoners. However, not only each situation differs considerably from its MinImps-based counterparts, but also there is a more visible difference between the effect on each of the case types. All four raincloud plots contain situations where the modularity made the number of reasoner calls increase. Still, the number of cases this these happens is proportionally small.

In cases of the type T1 (a contraction of subsumption), for both environments and reasoners, the mean and median are between 3 and $4 \log 2 \mathrm{FC}$, slightly worse than the value for MinImps. The first and third quartiles also show similar depreciation. Also, the peak of the kernel density estimation is close to 1 . However, the probability density is still high between $\log 2 \mathrm{FC} 3$ and 7 , similar to the case for MinImps.

As for instances regarding inconsistencies (type T2), the fold change improves instead of worsening when compared to the other class of cases, in contrast to what happens with MinImps. For all reasoners and environments, the mean and median were very close to 4 , and the range between first and third quartile moved from roughly $[1,5]$ to around $[2,6]$. Also, all cases have peaks of estimate probability at 5 (although another closer peak is seen at 1.5). Moreover, there are fewer cases where modularity increased the number of reasoner calls. Further, there is a more significant proportion of cases that display more substantial gains ( $\log 2 \mathrm{FC}$ above 7 ), even when comparing with the MinImps counterparts.

A question related to Q2 consists of determining whether MinImps-based outperform MaxNonsbased ones. Concerning the number of reasoner calls, Figure 6.7 shows the distribution of $\log 2 \mathrm{FC}$ for checker calls of Basic-MN over Basic-MI (higher log2FC indicate improvement when using Basic$\mathrm{MN})$.

For the cases of type T1, even though the median is above 0 , there is a slight concentration of 
case type $=\mathrm{T} 1$
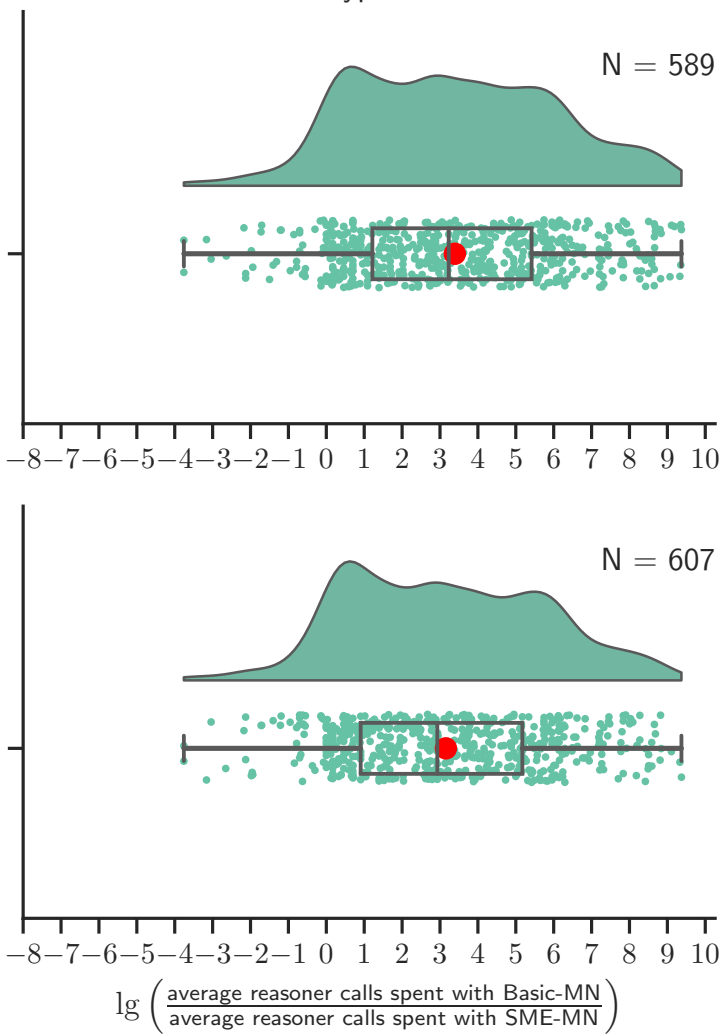

case type $=\mathrm{T} 2$
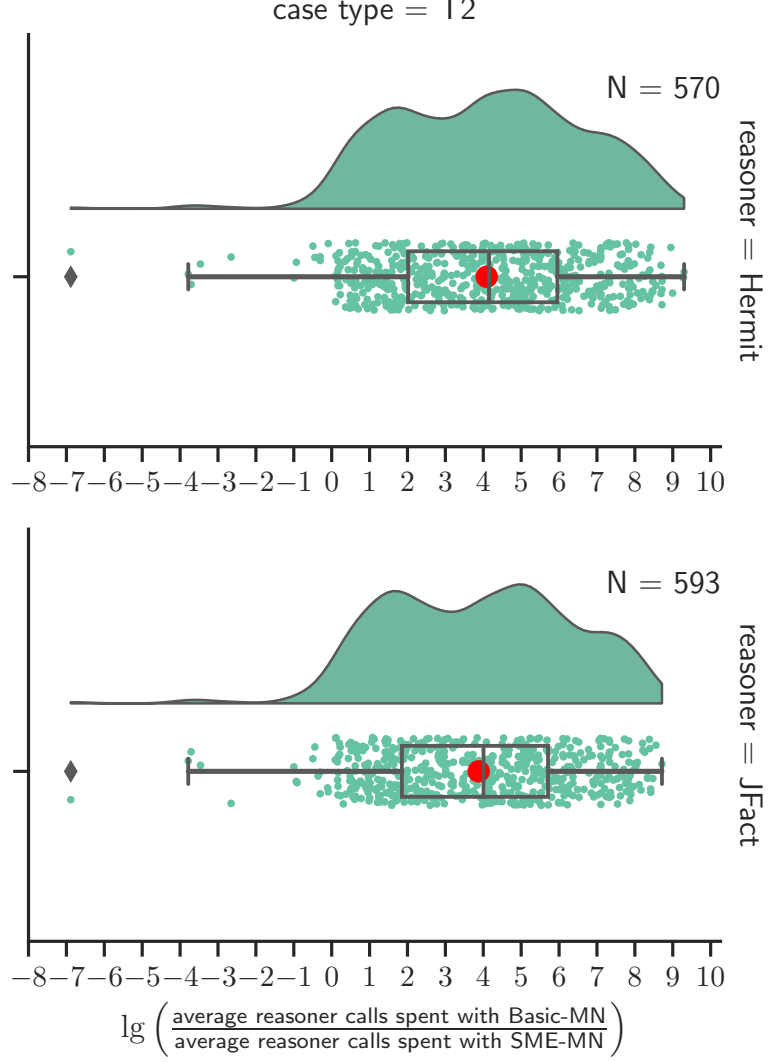

Figure 6.6: Distribution of log2FC for reasoner calls spent in average by Basic-MN and SME-MN in Full-M2

case type $=\mathrm{T} 1$
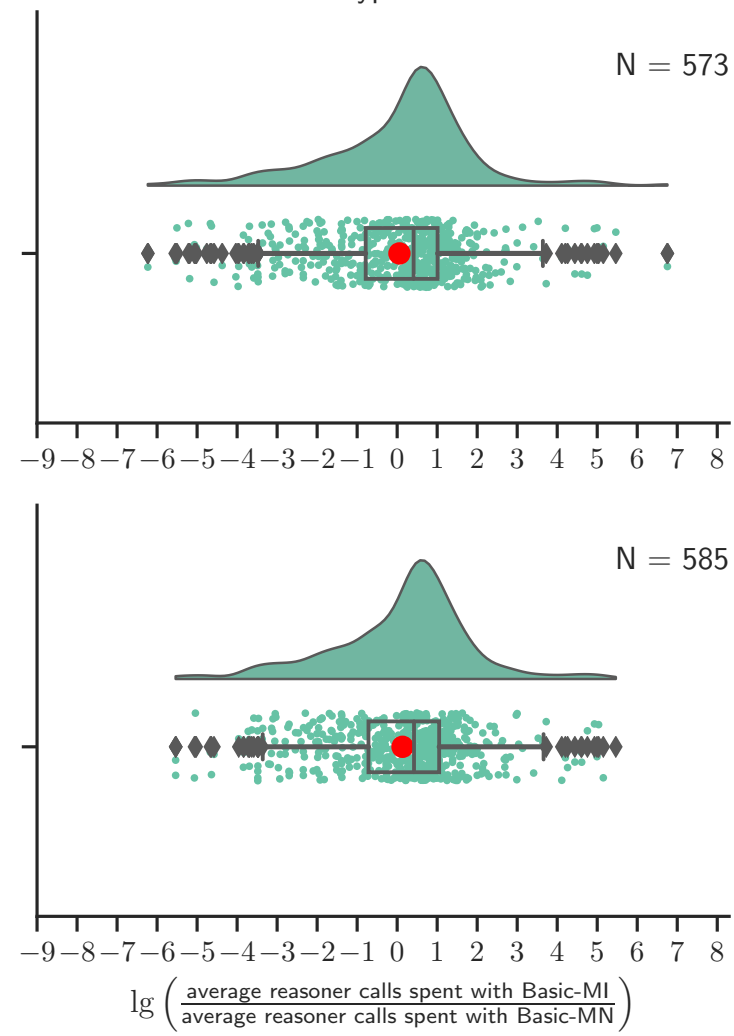
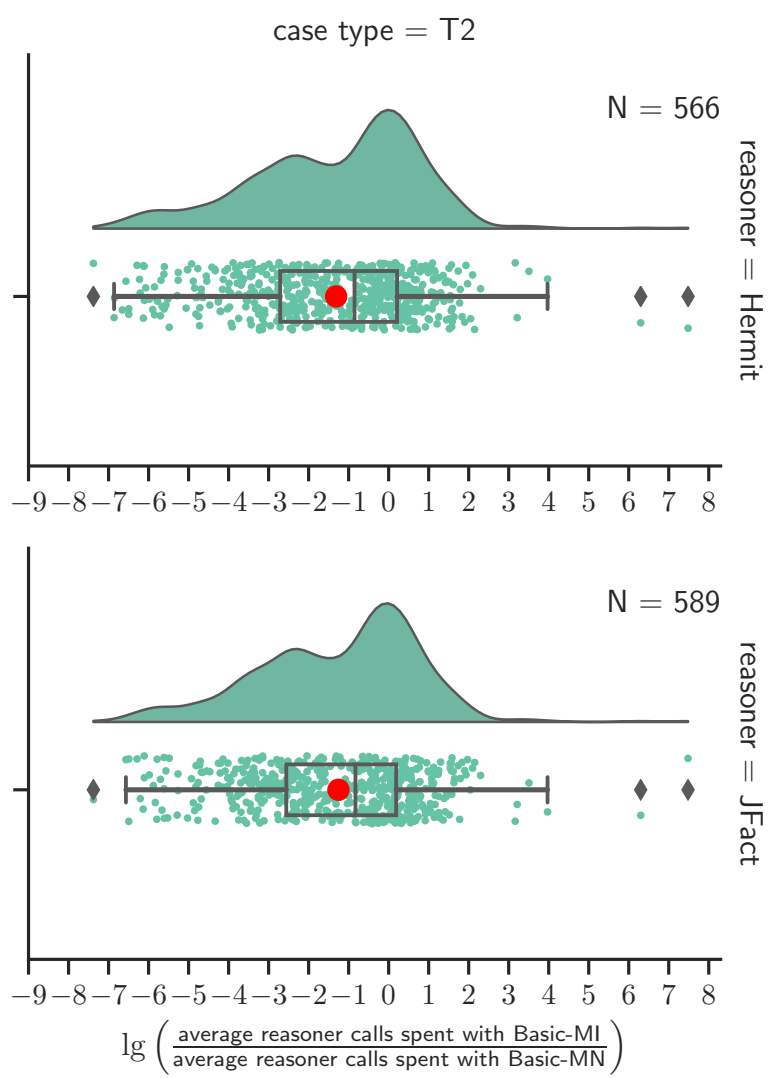

Figure 6.7: Distribution of log2FC for reasoner calls spent in average by Basic-MI and Basic-MN in Full-M2 
observations where Basic-MN fared worse than Basic-MI. The means does not help in the decision since it is almost 0 for both reasoners. In the cases of type T2, Figure 6.7 indicates that Basic-MI is generally better in the number of reasoner calls. The higher expected density at negative values of $\log 2 \mathrm{FC}$ and the fact that both the median and mean are negative support this observation. As expected, no noteworthy differences between reasoners occur.

Figure 6.8 is analogous to Figure 6.7, but with both constructions computed using single module extraction. The effect is that the MaxNons approach loses even more. In all four situations, even the mean and third quartile are negative. In very few observations, SME-MN displayed a better number of checker calls, and more than $25 \%$ of the inputs were four times or more costly with SME-MN than with SME-MI.
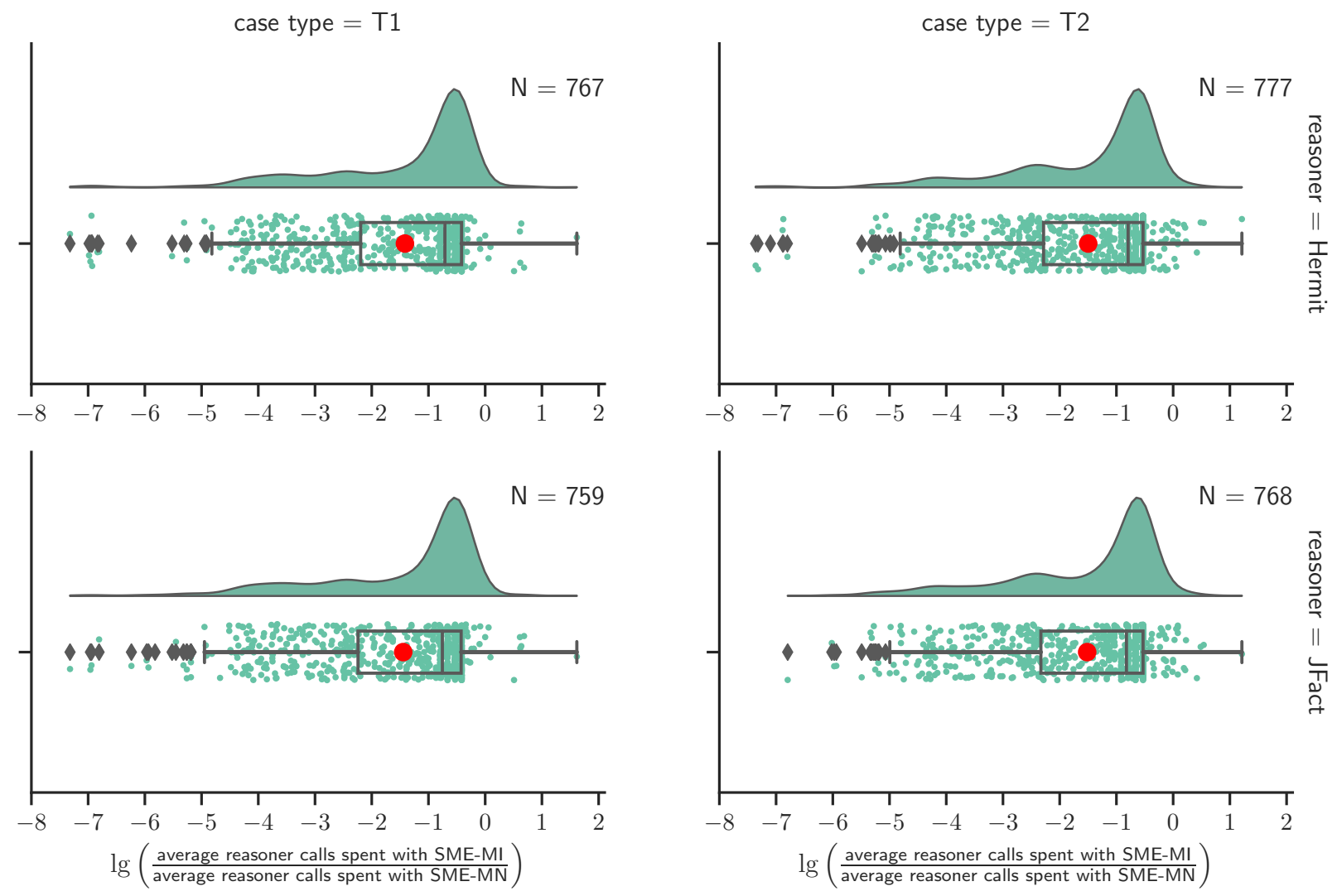

Figure 6.8: Distribution of log2FC for reasoner calls spent in average by SME-MI and SME-MN in Full-M2

Note that Figures 6.5 to 6.8 only refer to Full-M2. Table 6.5 displays the summarised statistics for $\log 2 \mathrm{FC}$ of checker calls between for the pairs of methods compared in Full-M1 and in FullM2. The similarity in values between these two experiments attests that the experiments are fairly stable.

\begin{tabular}{cclcccccccc}
\hline From & To & Experiment & $\mathrm{N}$ & Mean & Std. & \multicolumn{1}{c}{ Min } & $25 \%$ & $50 \%$ & $75 \%$ & Max \\
\hline \multirow{2}{*}{ Basic-MI } & \multirow{2}{*}{ SME-MI } & Full-M1 & 1779 & 4.5091 & 2.1057 & 0.2451 & 2.8524 & 4.1358 & 6.0622 & 10.9477 \\
& & Full-M2 & 2628 & 4.4785 & 2.0585 & 0.2451 & 2.8736 & 4.0732 & 5.9528 & 10.9474 \\
Basic-MN & \multirow{2}{*}{ SME-MN } & Full-M1 & 1630 & 3.6367 & 2.6478 & -6.8844 & 1.5236 & 3.6473 & 5.6473 & 10.4864 \\
& & Full-M2 & 2359 & 3.6260 & 2.5775 & -6.8844 & 1.5850 & 3.6554 & 5.5380 & 9.3710 \\
\multirow{2}{*}{ Basic-MI } & \multirow{2}{*}{ Basic-MN } & Full-M1 & 1590 & -0.5904 & 2.0632 & -7.3791 & -1.8688 & -0.1445 & 0.6804 & 7.4839 \\
& & Full-M2 & 2313 & -0.5934 & 2.0361 & -7.3791 & -1.9208 & -0.1476 & 0.7063 & 7.4842 \\
\multirow{2}{*}{ SME-MI } & \multirow{2}{*}{ SME-MN } & Full-M1 & 2058 & -1.4759 & 1.3713 & -7.5780 & -2.2869 & -0.7935 & -0.5305 & 1.2120 \\
& & Full-M2 & 3071 & -1.4688 & 1.3503 & -7.3607 & -2.2550 & -0.7935 & -0.5305 & 1.6158 \\
\hline
\end{tabular}

Table 6.5: Summarised statistics of reasoner calls log2FC per method pair in Full-M1 and Full-M2 


\section{Processing Time}
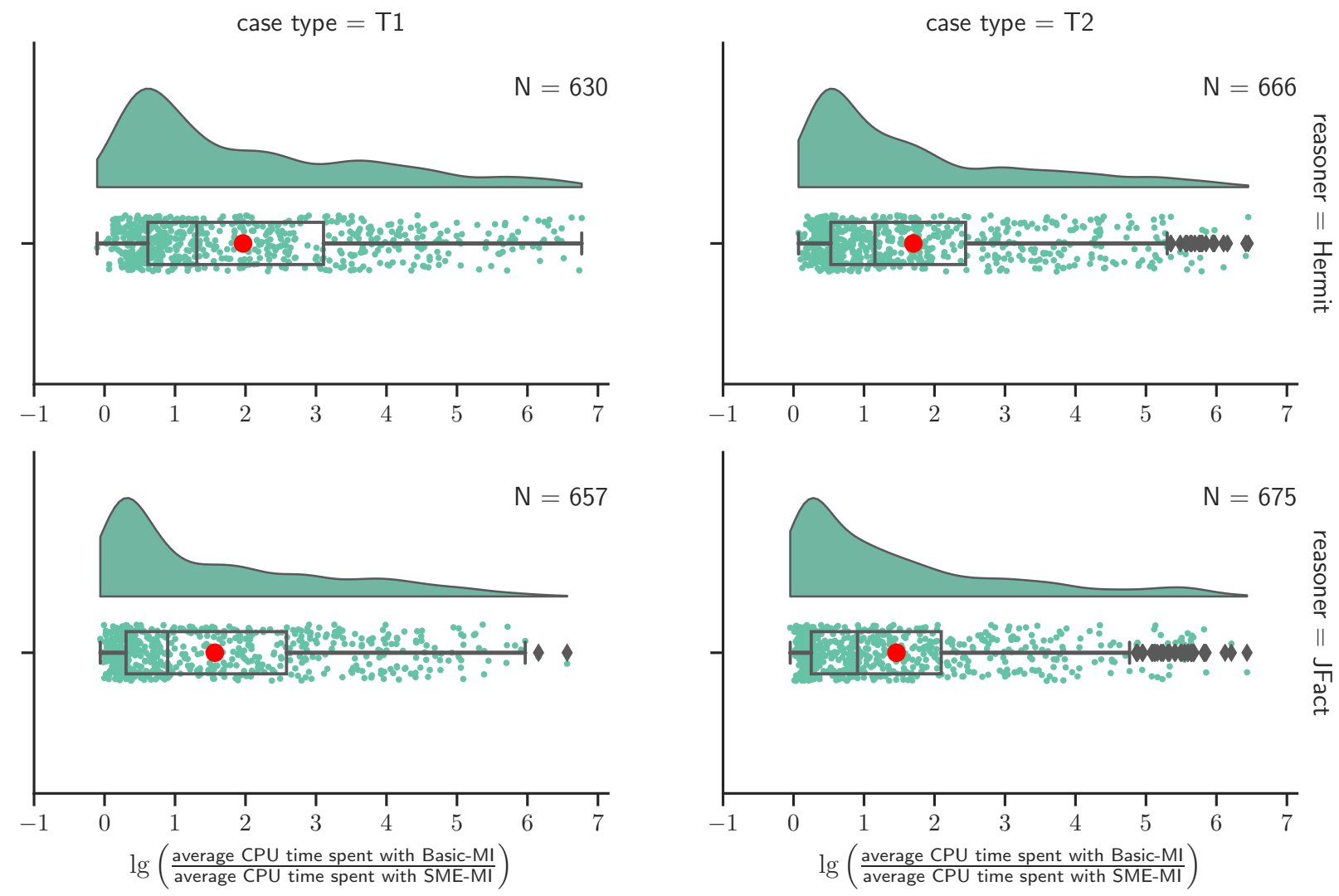

Figure 6.9: Distribution of log2FC for CPU time spent in average by Basic-MI and SME-MI in Full-M2

Figure 6.9 displays the distribution of fold changes in processing time for MinImps computation with and without modularity in Full-M2 (that is the change from going from Basic-MI to SME-MI). In all cases, modularity either improves the time consumption or keeps it the same. A large portion of runs had $\log 2 \mathrm{FC}$ from 0 to 1 , that is, many cases were only up to two times faster when using SME. Moreover, the means in all eight raincloud plots are between $\log 2 \mathrm{FC} 1$ and 2. Particularly for the cases of type 1, Hermit displayed a slight improvement with modularity as seen that the third quartile is higher for this reasoner than for JFact. In summary, the improvement in fold change for processing time is worse than the fold change in the number of reasoners calls; one possible explanation is due to overhead in computing modules.

As Figure 6.10 depicts, the effect of modularisation on processing time for MaxNons is akin to that of MinImps. There is a concentration of observations that stayed the same or were up to two times faster with modularity. Here, similarly to the case for reasoner calls, there are a few situations where the modularised algorithm was worse than the classical one. Another interesting observation is concerning the inputs of case type T2 (the inconsistent): the means of $\log 2 \mathrm{FC}$ was visibly better than in the MinImps case. With Hermit, for instance, the $\log 2 \mathrm{FC}$ was well above 2 . While this fact does not tell anything regarding MinImps versus MaxNons, it indicates that for this case type the impact of modularity was stronger in the MaxNons approach.

Figure 6.11 shows the raincloud plots comparing the classical MaxNons (Basic-MN) approach with the classical MinImps one in Full-M2 (Basic-MI), while Figure 6.12 does the same for the modularised ones (change from SME-MN to SME-MI). In both cases, the effects seen with reasoner calls are dampened, although the trend is the same. In all raincloud plots, the centrality measures and peaks of the estimated probability are in the interval $[-1,0]$. The only notable change between the classical and modularised versions is that, for the latter, the values are even more concentrated around the mean and median indicating a sharper improvement for the MaxNons approach when 

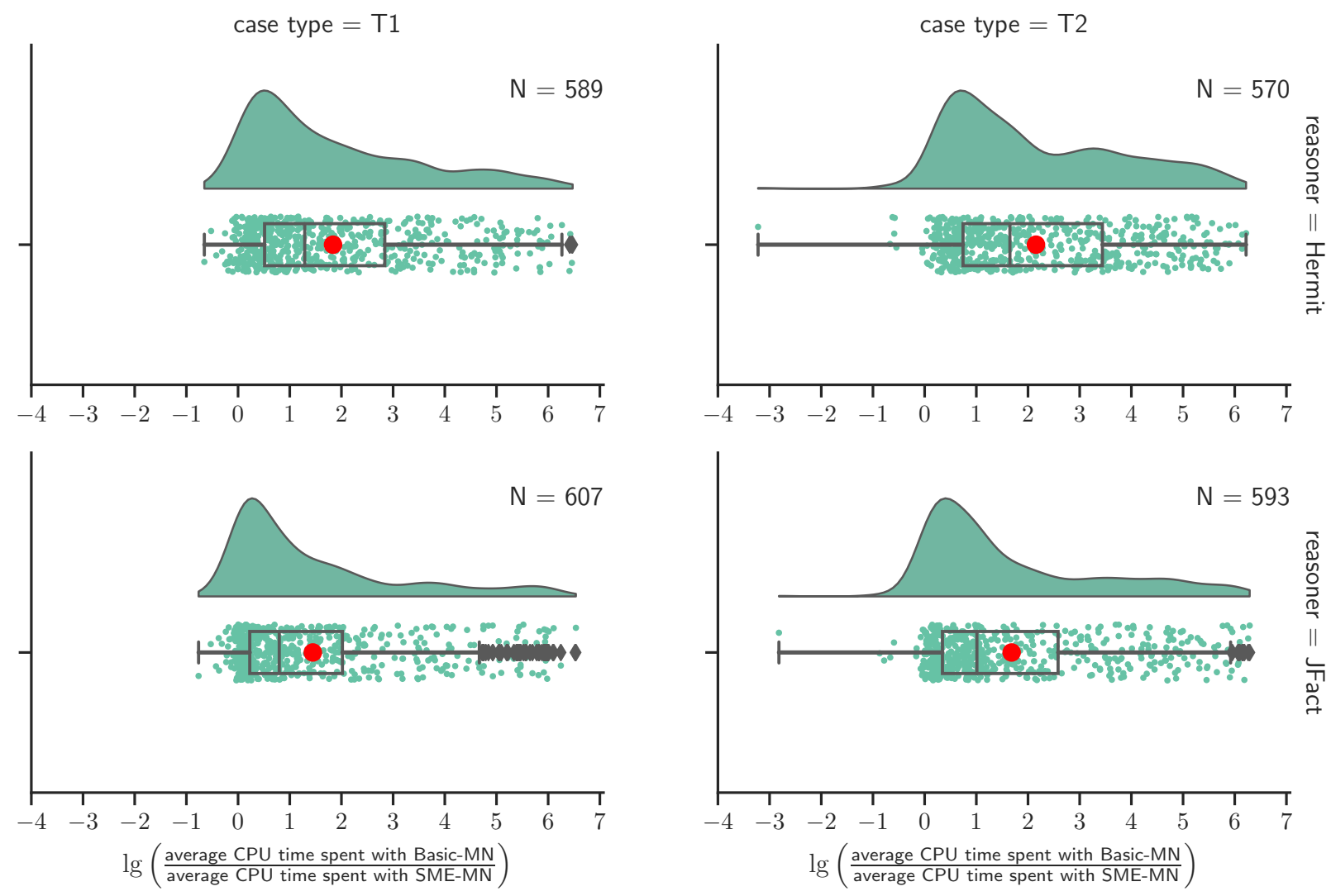

Figure 6.10: Distribution of log2FC for CPU time spent in average by Basic-MN and SME-MN in Full-M2

compared to the MinImps one.

Table 6.6 summarises the fold changes for processing time and includes the results for Full-M1. Again, the similarity between the fold changes in both experiments attests to the effectiveness of the experimental design and environment preparation.

\begin{tabular}{cclcccccccc}
\hline From & To & Experiment & \multicolumn{1}{c}{ N } & Mean & \multicolumn{1}{c}{ Std. } & \multicolumn{1}{c}{ Min } & $25 \%$ & \multicolumn{1}{c}{$50 \%$} & \multicolumn{1}{c}{$75 \%$} & Max \\
\hline Basic-MI & \multirow{2}{*}{ SME-MI } & Full-M1 & 1779 & 1.6626 & 1.6078 & -0.0333 & 0.3655 & 1.0344 & 2.6046 & 6.9167 \\
& & Full-M2 & 2628 & 1.6735 & 1.5682 & -0.1048 & 0.4224 & 1.0832 & 2.5608 & 6.7700 \\
Basic-MN & \multirow{2}{*}{ SME-MN } & Full-M1 & 1630 & 1.8051 & 1.7614 & -2.9736 & 0.3814 & 1.1386 & 2.9556 & 7.0355 \\
& & Full-M2 & 2359 & 1.7822 & 1.6992 & -3.2227 & 0.4447 & 1.1532 & 2.8571 & 6.5366 \\
Basic-MI & \multirow{2}{*}{ Basic-MN } & Full-M1 & 1590 & -0.6828 & 1.1264 & -5.3586 & -0.9748 & -0.3133 & -0.0063 & 2.7772 \\
& & Full-M2 & 2313 & -0.6727 & 1.1119 & -5.2232 & -0.9886 & -0.3292 & -0.0051 & 2.8625 \\
SME-MI & \multirow{2}{*}{ SME-MN } & Full-M1 & 2058 & -0.4691 & 0.7770 & -5.8077 & -0.5584 & -0.1263 & -0.0405 & 1.8114 \\
& & Full-M2 & 3071 & -0.4780 & 0.7739 & -5.3909 & -0.5649 & -0.1472 & -0.0548 & 1.7058 \\
\hline
\end{tabular}

Table 6.6: Summarised statistics of processing time log2FC per method pair in Full-M1 and Full-M2

\section{Memory}

The third quantity evaluated is memory consumption, measured via the highest watermark of virtual memory, as discussed in Section 5.2. Figure 6.13 shows the effect of modularity on MinImps computation. Again, the fold changes shown indicate the transition from Basic-MI to SME-MI. For this metric Full-M1 and Full-M2 differ significantly. Hence the presentation of raincloud plots for both environments.

According to the graphs in Figure 6.13a, many executions in Full-M1 have little variation in memory spent. However, another representative group required $25 \%$ or less memory with modularity. 
case type $=\mathrm{T} 1$
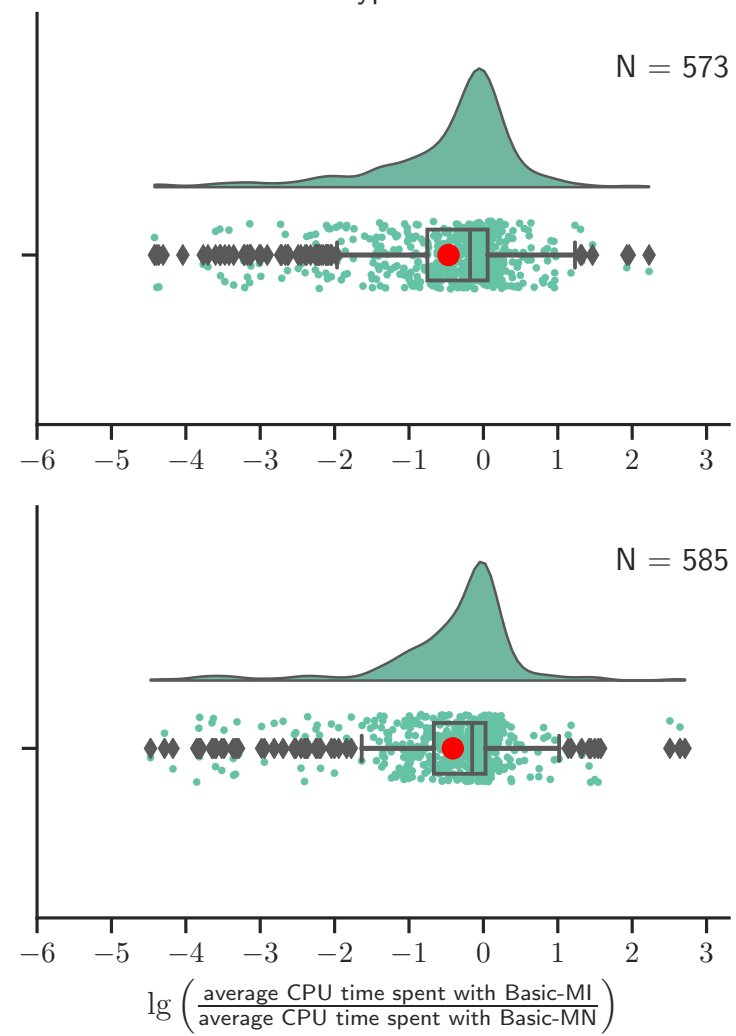

case type $=\mathrm{T} 2$
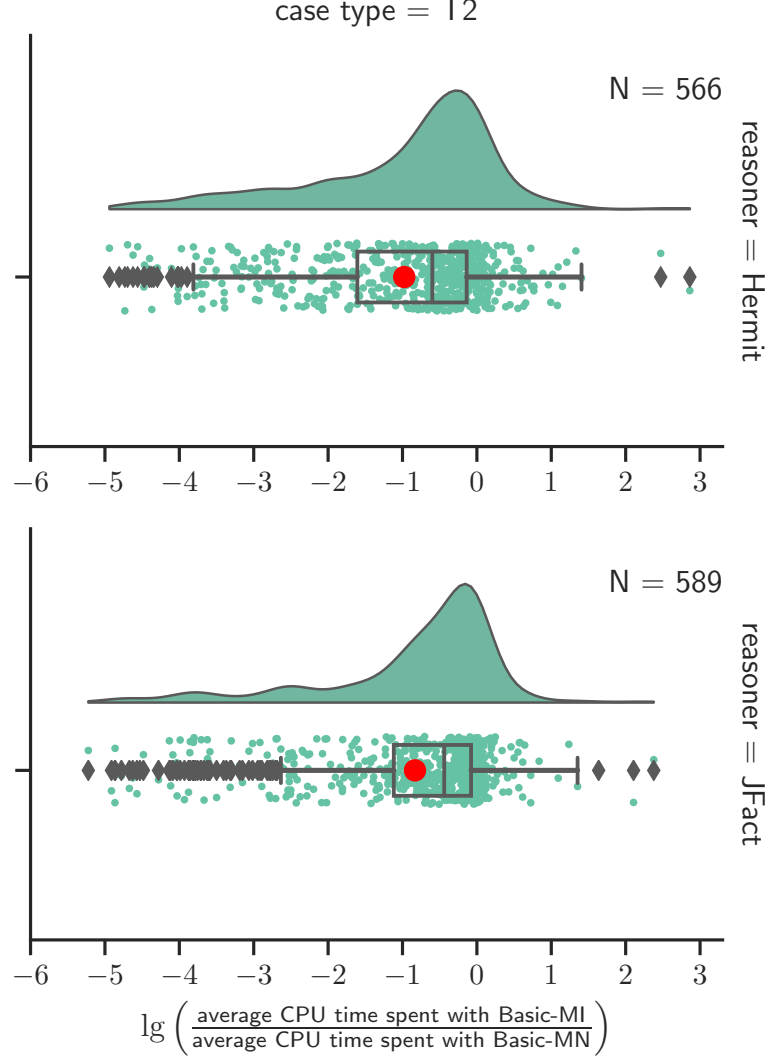

Figure 6.11: Distribution of log2FC for CPU time spent in average by Basic-MI and Basic-MN in Full-M2
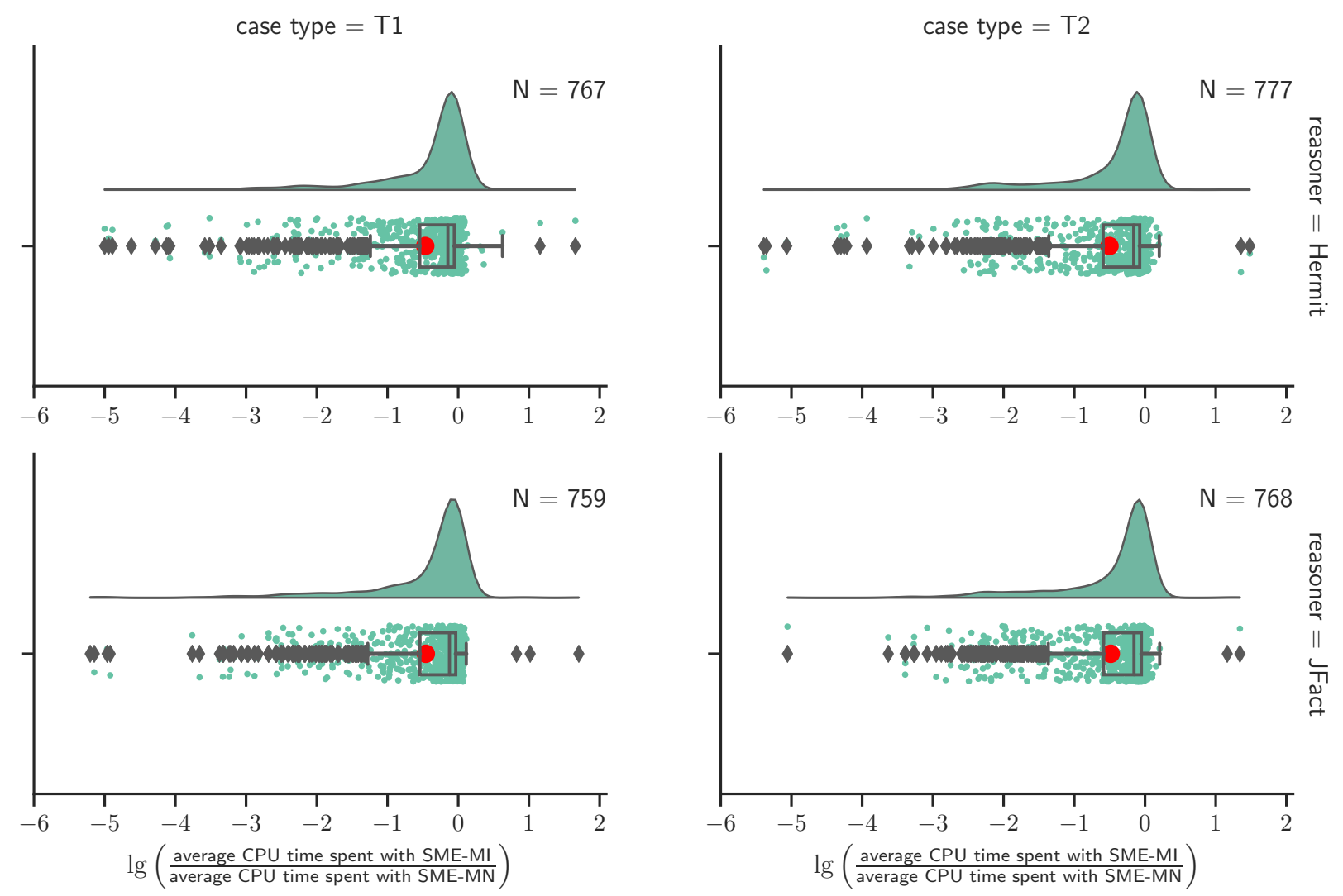

Figure 6.12: Distribution of log2FC for CPU time spent in average by SME-MI and SME-MN in Full-M2 

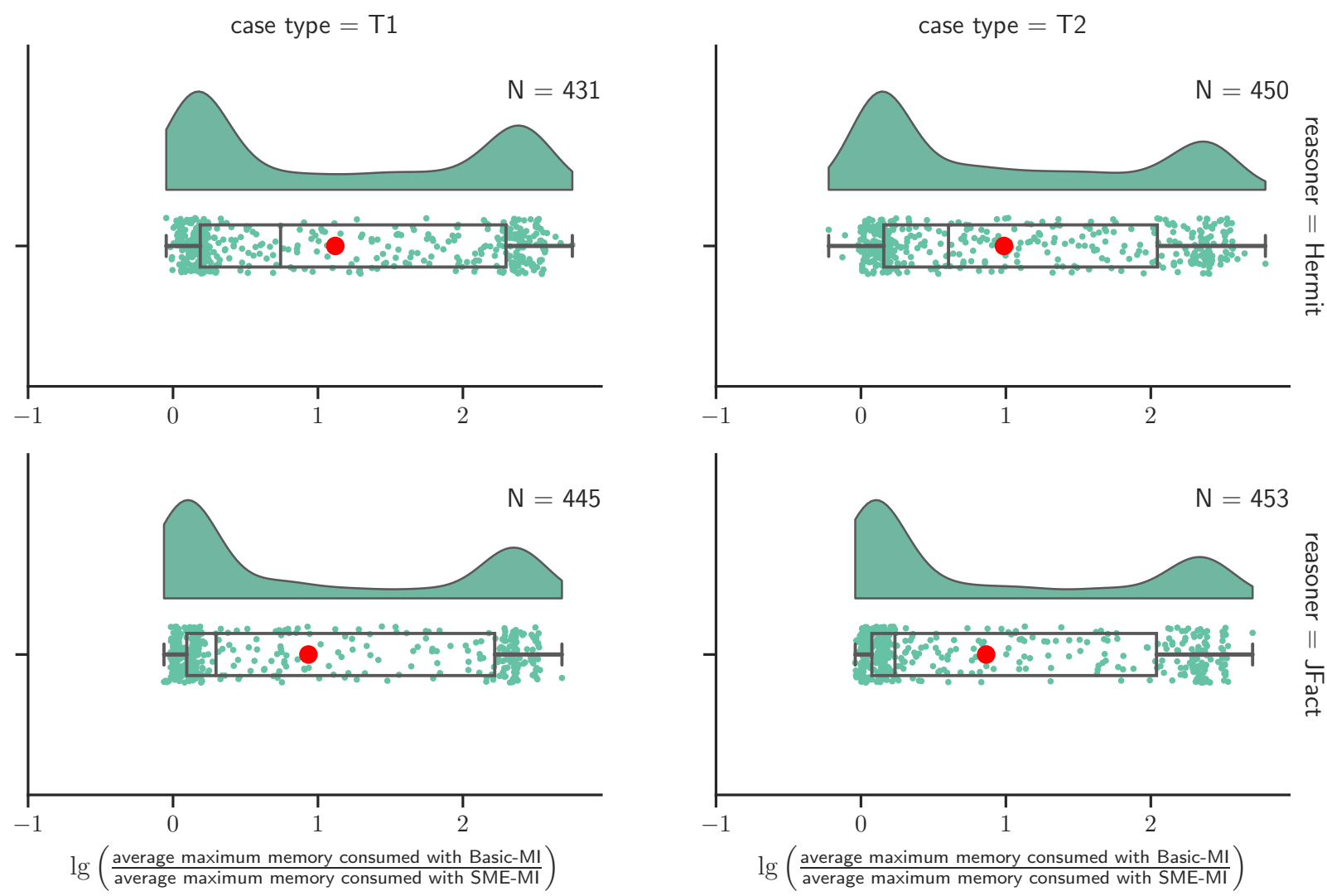

(a) Raincloud plot for Full-M1
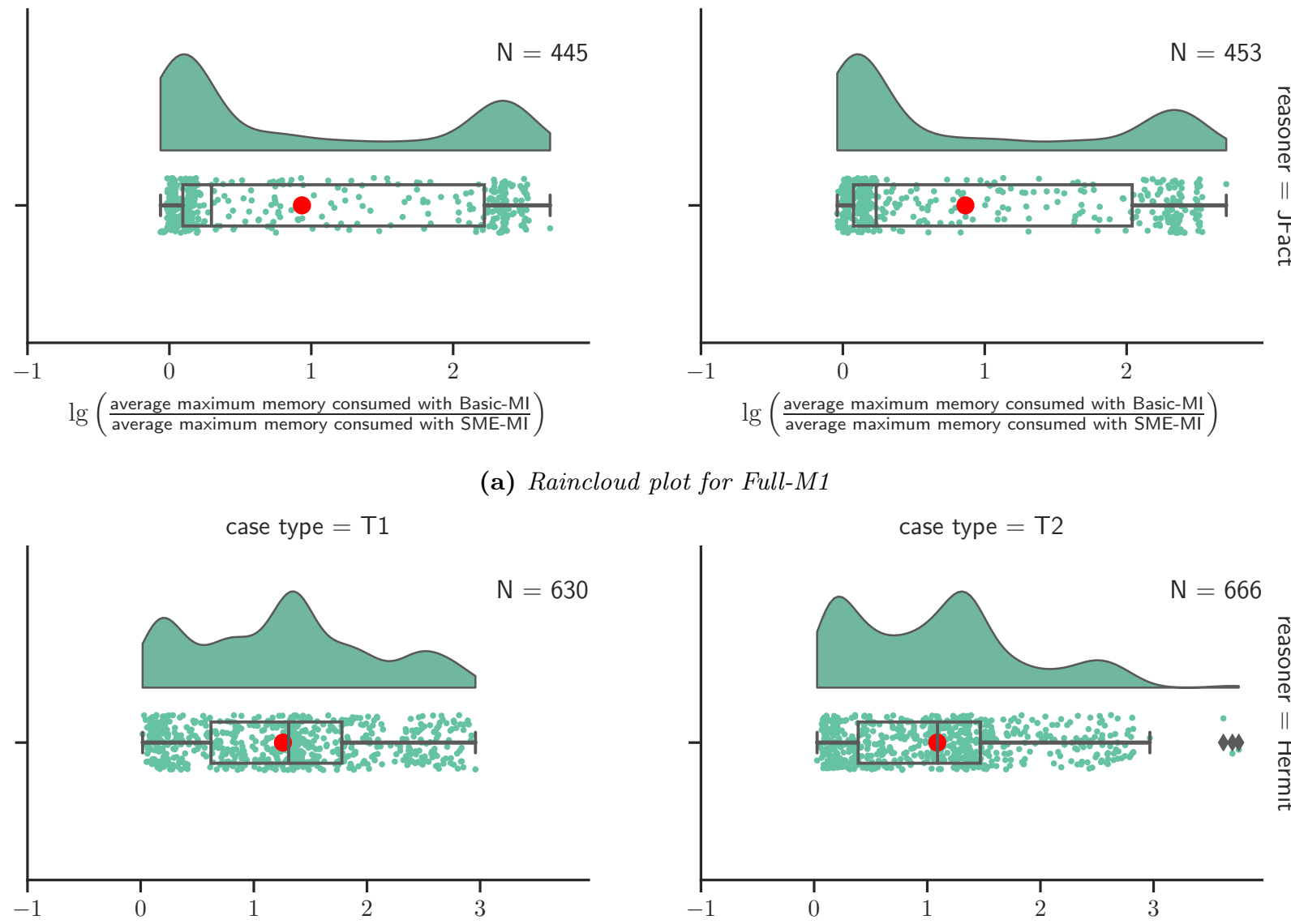

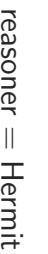
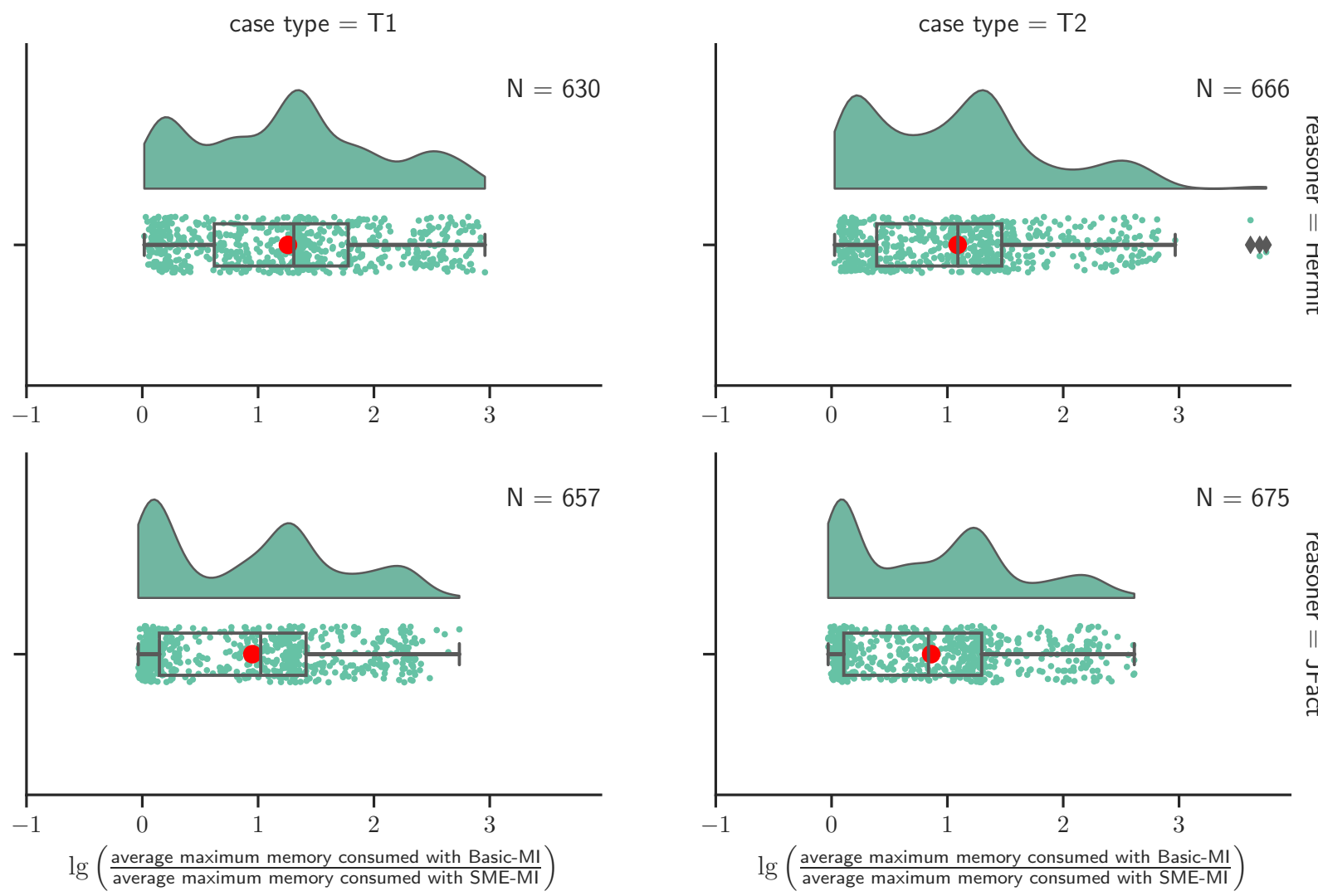

(b) Raincloud plot for Full-M2

Figure 6.13: Distribution of maximum memory (VmHWM) consumed in average by Basic-MI and SME-MI 
For Full-M2, Figure 6.13b shows three main groups: those who had almost no change, those that cut memory spent a bit less than half of the original memory and finally a smaller and more sparse group with $\log 2 \mathrm{FC}$ between 2 and 3 . In all situations, the trends across reasoners and case types are roughly the same.

The same effects can be observed in MaxNons (from Basic-MN to SME-MN) as Figure 6.14 depicts, albeit with more observations where the memory consumption increased with modularity (still a very small portion).

As for the comparison between MaxNons and MinImps-based approaches concerning memory, Figures 6.15 and 6.16 show a similar panorama as in task clock evaluation. MaxNons-based methods generally perform worse than MinImps-bases ones, but most of the observations do not display significant changes (the centrality measures and density are close to 0). As the distributions are similar for Full-M1 and Full-M2, the plot for the former is omitted.

\section{Timeouts}

Up to now, the evaluation only considered inputs where all the executions in a group finished for both methods. Hence, the groups of executions (repetitions which the same combination of parameters) displaying timeouts were overlooked. These groups, however, contain valuable information as it is possible to compare how many failures one method had that another did not. In this sense, Tables 6.7 and 6.8 count the groups of executions per timeout status for each method in Full-M1 and Full-M2, respectively (a group is the set of repetitions of a method for the same case input and reasoner combination).

\begin{tabular}{lrrrr}
\hline Method & Fully successful & Only timeouts & Some timeout & Success rate \\
\hline Basic-MI & 1781 & 393 & 395 & $80.08 \%$ \\
SME-MI & 2137 & 57 & 57 & $98.09 \%$ \\
MU-MI & 2137 & 57 & 57 & $98.09 \%$ \\
Basic-MN & 1635 & 556 & 559 & $73.52 \%$ \\
SME-MN & 2076 & 135 & 136 & $93.35 \%$ \\
\hline
\end{tabular}

Table 6.7: Number of group of executions per timeout status and method (2224 groups) in Full-M1

\begin{tabular}{lrrrr}
\hline Method & Fully successful & Only timeouts & Some timeout & Success rate \\
\hline Basic-MI & 2631 & 617 & 627 & $79.06 \%$ \\
SME-MI & 3195 & 90 & 91 & $96.00 \%$ \\
MU-MI & 3194 & 90 & 92 & $95.97 \%$ \\
Basic-MN & 2368 & 884 & 914 & $71.15 \%$ \\
SME-MN & 3095 & 213 & 215 & $93.00 \%$ \\
\hline
\end{tabular}

Table 6.8: Number of group of executions per timeout status and method (3328 groups) in Full-M2

In both experiments, Basic-MN has the lowest success rate, being followed by Basic-MI. The modularity approaches improved the success rate considerably. After modularisation, the MaxNonsbased method still produces more timeouts than their MinImps counterparts. Concerning termination status, SME-MI and MU-MI can be considered equivalent: in Full-M1 they have the same success rate, and in Full-M2 they differ only by a single group of observations that had some (i.e. some repetitions were successful) timeouts for the module update version. In fact, the actual data for the timeout tables shows that in Full-M1, SME-MI and MU-MI are timeout-free on the same groups of executions and in all but one in Full-M2. The date also shows that there are groups that are timeout-free without modularity, but had timeouts with modularity. Still, the gains seem to compensate for the eventual losses. 
Excluding timeouts other reasons that prevented successful termination include: stack overflow errors (too many nested recursive calls) and failures in parsing ontologies (e.g. due to unsupported datatypes).

\section{Optimality Rate}

Another way to compare the methods is to count, for a specific quantity, how many times each method produced the minimum. Here, this metric is called optimality rate and corresponds, for a given method, to quantity or proportion of the entirely successful execution groups (no timeouts for any method) for which that method was close enough to the minimum. This margin is of $5 \%$ of the minimum to account for potential noise. For instance, in Full-M1, SME-MI had average reasoner calls between the minimum and $1.05 \times$ minimum for 2020 groups of execution, which corresponds to $90.83 \%$ of the groups. Tables 6.9 and 6.10 lists the number of optimal cases and rates per quantity and method in Full-M1 and Full-M2, respectively.

\begin{tabular}{lrrrrrr}
\hline \multirow{2}{*}{ Method } & \multicolumn{2}{c}{ Reasoner calls } & \multicolumn{2}{c}{ Processing time } & \multicolumn{2}{c}{ Memory } \\
& Groups & \multicolumn{1}{c}{$\%$} & Groups & $\%$ & Groups & $\%$ \\
\hline Basic-MI & 0 & $0.00 \%$ & 34 & $1.53 \%$ & 267 & $12.01 \%$ \\
SME-MI & 2020 & $90.83 \%$ & 1907 & $85.75 \%$ & 1941 & $87.28 \%$ \\
MU-MI & 2091 & $94.02 \%$ & 2037 & $91.59 \%$ & 1988 & $89.39 \%$ \\
Basic-MN & 30 & $1.35 \%$ & 51 & $2.29 \%$ & 228 & $10.25 \%$ \\
SME-MN & 52 & $2.34 \%$ & 751 & $33.77 \%$ & 1164 & $52.34 \%$ \\
\hline
\end{tabular}

Table 6.9: Number and percentage of executions per method that achieve the smallest (best) value in each quantity within a margin of $5 \%$ from the actual minimum in Full-M1

\begin{tabular}{lrcrrrr}
\hline \multirow{2}{*}{ Method } & \multicolumn{2}{c}{ Reasoner calls } & \multicolumn{2}{c}{ Processing time } & \multicolumn{2}{c}{ Memory } \\
& Groups & \multicolumn{1}{c}{$\%$} & Groups & $\%$ & Groups & \multicolumn{1}{c}{$\%$} \\
\hline Basic-MI & 0 & $0.00 \%$ & 31 & $0.93 \%$ & 153 & $4.60 \%$ \\
SME-MI & 3016 & $90.63 \%$ & 2636 & $79.21 \%$ & 2795 & $83.98 \%$ \\
MU-MI & 3136 & $94.23 \%$ & 2790 & $83.83 \%$ & 3039 & $91.32 \%$ \\
Basic-MN & 40 & $1.20 \%$ & 54 & $1.62 \%$ & 172 & $5.17 \%$ \\
SME-MN & 72 & $2.16 \%$ & 686 & $20.61 \%$ & 1292 & $38.82 \%$ \\
\hline
\end{tabular}

Table 6.10: Number and percentage of executions per method that achieve the smallest (best) value in each quantity within a margin of $5 \%$ from the actual minimum in Full-M2

In Tables 6.9 and 6.10, the modularised versions dominate the non-modularised ones in all quantities. Basic-MN is better than Basic-MI in every case listed, however, SME-MN always loses to SME-MI and MU-MI. Finally, MU-MI dominates by a small margin over SME-MI indicating that the incremental strategy of Algorithm 13 provides benefits even if small ones.

\section{Correlation}

Now it is interesting to look at the possible factors that imply on the fold changes studied. Tables 6.11 and 6.12 present some correlations between the fold changes presented before and the number of axioms of an ontology, the value of the quantity and the module's relative size. For each comparison they display the degree of association calculated via Kendall's $\tau$ and the p-value. As discussed, the closer the absolute value of correlation to 1 or -1 , the stronger is the association, while the signal indicates whether it is positive or negative. The p-value indicates, roughly, the chance that such degree would be observed due to chance alone (hence, smaller is better). 


\begin{tabular}{|c|c|c|c|c|c|c|}
\hline \multirow[t]{2}{*}{ From } & \multirow[t]{2}{*}{ To } & \multirow[t]{2}{*}{$\mathrm{N}$} & \multirow[t]{2}{*}{ Quantity } & \multicolumn{3}{|c|}{ Correlation (Kendall's $\tau$ ) } \\
\hline & & & & $\begin{array}{l}\text { Ontology size } \\
\text { (axioms) }\end{array}$ & $\begin{array}{l}\text { Average for } \\
\text { "from" }\end{array}$ & $\begin{array}{l}\text { Relative module } \\
\text { size }\end{array}$ \\
\hline \multirow[t]{3}{*}{ Basic-MI } & SME-MI & 1779 & Reasoner calls & 0.3605 & 0.6371 & -0.1872 \\
\hline & & & CPU time & 0.5496 & 0.8628 & -0.1668 \\
\hline & & & Memory & 0.5062 & 0.7808 & -0.1783 \\
\hline \multirow[t]{3}{*}{ Basic-MI } & MU-MI & 1779 & Reasoner calls & 0.3609 & 0.6395 & -0.1865 \\
\hline & & & CPU time & 0.5539 & 0.8622 & -0.1732 \\
\hline & & & Memory & 0.5125 & 0.7862 & -0.1821 \\
\hline \multirow[t]{3}{*}{ Basic-MN } & SME-MN & 1630 & Reasoner calls & 0.3428 & 0.4562 & -0.5728 \\
\hline & & & CPU time & 0.5879 & 0.7623 & -0.3057 \\
\hline & & & Memory & 0.4644 & 0.6289 & -0.3458 \\
\hline \multirow[t]{3}{*}{ Basic-MI } & Basic-MN & 1590 & Reasoner calls & -0.0719 & 0.1518 & 0.1217 \\
\hline & & & CPU time & -0.3042 & -0.1438 & 0.1503 \\
\hline & & & Memory & -0.1140 & 0.0860 & 0.0884 \\
\hline \multirow[t]{3}{*}{ SME-MI } & SME-MN & 2058 & Reasoner calls & -0.1607 & -0.6373 & -0.3316 \\
\hline & & & CPU time & -0.3526 & -0.5548 & -0.1300 \\
\hline & & & Memory & -0.2563 & -0.2669 & -0.1521 \\
\hline
\end{tabular}

Table 6.11: Correlations between the fold changes and ontology size, value of the quantities measured and the relative size of the module for Full-M1 (largest p-value: $1.7999 \times 10^{-5}$ )

\begin{tabular}{|c|c|c|c|c|c|c|}
\hline \multirow[t]{2}{*}{ From } & \multirow[t]{2}{*}{ To } & \multirow[t]{2}{*}{$\mathrm{N}$} & \multirow[t]{2}{*}{ Quantity } & \multicolumn{3}{|c|}{ Correlation (Kendall's $\tau$ ) } \\
\hline & & & & $\begin{array}{l}\text { Ontology size } \\
\text { (axioms) }\end{array}$ & $\begin{array}{l}\text { Average for } \\
\text { "from" }\end{array}$ & $\begin{array}{l}\text { Relative module } \\
\text { size }\end{array}$ \\
\hline \multirow[t]{3}{*}{ Basic-MI } & SME-MI & 2628 & Reasoner calls & 0.3508 & 0.6282 & -0.1886 \\
\hline & & & CPU time & 0.5173 & 0.8222 & -0.1498 \\
\hline & & & Memory & 0.4869 & 0.7133 & -0.2106 \\
\hline \multirow[t]{3}{*}{ Basic-MI } & MU-MI & 2628 & Reasoner calls & 0.3508 & 0.6307 & -0.1878 \\
\hline & & & $\mathrm{CPU}$ time & 0.5193 & 0.8208 & -0.1543 \\
\hline & & & Memory & 0.5030 & 0.7199 & -0.2288 \\
\hline \multirow[t]{3}{*}{ Basic-MN } & SME-MN & 2359 & Reasoner calls & 0.3409 & 0.4524 & -0.5784 \\
\hline & & & CPU time & 0.5704 & 0.7311 & -0.3144 \\
\hline & & & Memory & 0.4508 & 0.5722 & -0.4676 \\
\hline \multirow[t]{3}{*}{ Basic-MI } & Basic-MN & 2313 & Reasoner calls & -0.0667 & 0.1576 & 0.1171 \\
\hline & & & CPU time & -0.2984 & -0.1240 & 0.1551 \\
\hline & & & Memory & -0.1632 & 0.0389 & 0.1382 \\
\hline \multirow[t]{3}{*}{ SME-MI } & SME-MN & 3071 & Reasoner calls & -0.1570 & -0.6355 & -0.3292 \\
\hline & & & CPU time & -0.2905 & -0.4624 & -0.1713 \\
\hline & & & Memory & -0.2688 & -0.3523 & -0.1575 \\
\hline
\end{tabular}

Table 6.12: Correlations between the fold changes and ontology size, value of the quantities measured and the relative size of the module for Full-M2 (largest p-value: $5.0993 \times 10^{-3}$ )

In Tables 6.11 and 6.12, the only strong correlations (absolute value at least 0.7 ) are for the average values for each quantity obtained without modularity. More specifically, for MinImps, there is a strong correlation between the time and memory spent by Basic-MI and the fold change obtained with modularity (both with SME-MI and MU-MI). As for MaxNons, a strong correlation is only 
observed between the processing time spent by Basic-MN and the fold change when using SME-MN instead. For memory, the analogous correlation is only moderate. In all these cases, the association is direct (positive).

The correlation between the number of reasoner calls with Basic-MI and the fold change obtained with modularity is moderate (from 0.5 to 0.7 ). So is the association between the number of axioms in the ontology and time and memory fold changes from Basic-MI to SME-MI or MU-MI. Another moderate correlation is seen between the processing time spent by Basic-MN and the gains with SME-MN (for memory the correlation was only mild). These are all positive (direct) relationships.

There are also interesting negative correlations such as between the fold changes when transitioning from SME-MI to SME-MN, which is moderate for the number of checker calls in both tables, and also for task clock in Table 6.11. Another moderate inverse relationship occurs between the module size and the fold change for reasoner calls from Basic-MN to SME-MN, indicating the possible relation between smaller modules and increased saving in reasoner calls.

\subsubsection{Construction of Single Solutions}

This subsection concerns the seconds part of the research question Q2 which aims to evaluate the impact of modularity when any solution for repair (or for belief change) is enough, regardless of which. To this end, for MinImps approaches, it is only necessary to compute the first closed path in Algorithm 1; for MaxNons approaches only the first node is needed. For the MinImps case, it is also interesting to evaluate whether depth-first (navigation using a stack) or breadth-first approach (navigation using a queue) produces a significant change in performance.

The evaluation of methods and implementations in this subsection uses the same quantities, metrics and presentation devices applied in Subsection 6.2.2.

The MaxNons-based methods should not differ regarding HST implementation since only the root node is computed. However, the experiments evaluate the MaxNons methods using queue and stacks separately. This decision was motivated to guarantee symmetry with the MinImps methods, to observe that in fact queue and stack do not affect MaxNons when computing a single node and to better evaluate the impact of each implementation on the MinImps. The results obtained show that even for MinImps-based approaches, there is very little difference between the implementations.

Table 6.13 shows the basic statistics of the $\log 2 \mathrm{FC}$ when "replacing" a queue by a stack.

\begin{tabular}{lclrrrrrrr}
\hline Method & $\mathrm{N}$ & Quantity & \multicolumn{1}{c}{ Mean } & Std & \multicolumn{1}{c}{ Min } & \multicolumn{1}{c}{$25 \%$} & \multicolumn{1}{c}{$50 \%$} & $75 \%$ & Max \\
\hline Basic-MI & \multirow{2}{*}{1713} & Reasoner calls & -0.0304 & 0.2432 & -2.4809 & -0.0002 & 0.0000 & 0.0000 & 0.7251 \\
& & CPU time & -0.0209 & 0.1809 & -2.4027 & -0.0335 & -0.0002 & 0.0335 & 0.6454 \\
& & Memory & -0.0047 & 0.0596 & -0.5128 & -0.0164 & -0.0004 & 0.0140 & 0.3233 \\
SME-MI & 2132 & Reasoner calls & -0.0381 & 0.2324 & -2.9062 & 0.0000 & 0.0000 & 0.0000 & 1.0004 \\
& & CPU time & -0.0280 & 0.1547 & -1.8194 & -0.0382 & -0.0054 & 0.0270 & 0.6579 \\
& & Memory & -0.0088 & 0.0690 & -0.7895 & -0.0238 & -0.0016 & 0.0181 & 0.4369 \\
MU-MI & \multirow{2}{*}{2192} & Reasoner calls & -0.0991 & 0.5530 & -4.0255 & 0.0000 & 0.0000 & 0.0000 & 2.1584 \\
& & CPU time & -0.0388 & 0.2270 & -2.4365 & -0.0420 & -0.0016 & 0.0316 & 0.7776 \\
& & Memory & -0.0302 & 0.2045 & -1.9434 & -0.0280 & -0.0018 & 0.0211 & 1.1639 \\
Basic-MN & 2090 & Reasoner calls & -0.0003 & 0.0258 & -0.7370 & 0.0000 & 0.0000 & 0.0000 & 0.2996 \\
& & CPU time & 0.0005 & 0.0457 & -0.2084 & -0.0280 & 0.0005 & 0.0303 & 0.1677 \\
& & Memory & -0.0007 & 0.0315 & -0.1741 & -0.0182 & -0.0010 & 0.0165 & 0.1715 \\
SME-MN & 2156 & Reasoner calls & 0.0000 & 0.0000 & 0.0000 & 0.0000 & 0.0000 & 0.0000 & 0.0000 \\
& & CPU time & 0.0012 & 0.0449 & -0.1995 & -0.0288 & 0.0019 & 0.0304 & 0.1910 \\
& & Memory & -0.0009 & 0.0313 & -0.1967 & -0.0184 & -0.0019 & 0.0160 & 0.1464 \\
\hline
\end{tabular}

Table 6.13: Statistics of the log2FC from Queue to Stack for each method in Single-M2 
In Table 6.13, the variations in the rows corresponding to MaxNons methods are entirely due to the inherent variation provoked by external noises and non-deterministic behaviour on reasoners and data structures. For the MinImps-based techniques the distribution of fold change is very close to zero (quartiles and mean). However, the means in all three metrics for these methods is negative. Further, the minimum values are more distant from the mean and median than the maximum, thus indicating a tiny advantage for queue approaches.

As in the results in Subsection 6.2.2, the module update strategy for MinImps does not differ much from the SME one. However, as displayed in Table 6.14, most of the cases exhibited an improvement, for all metrics. Furthermore, the maximum gains attained are higher than in Full-M1 and Full-M2. Hence, in this subsection, SME-MI will be omitted in most of the discussions in favour of MU-MI.

\begin{tabular}{lccccccccc}
\hline Structure & $\mathrm{N}$ & Quantity & Mean & Std & \multicolumn{1}{c}{ Min } & \multicolumn{1}{c}{$25 \%$} & $50 \%$ & $75 \%$ & Max \\
\hline Queue & 22134 & Reasoner calls & 0.6547 & 1.0128 & 0.0000 & 0.2065 & 0.3626 & 0.4475 & 6.7733 \\
& & Processing time & 0.2004 & 0.5729 & -0.3580 & -0.0022 & 0.0417 & 0.1139 & 4.9568 \\
& & Memory & 0.1529 & 0.3374 & -0.2843 & -0.0011 & 0.0314 & 0.1065 & 2.3402 \\
\multirow{2}{*}{ Stack } & \multirow{2}{*}{2132} & Reasoner calls & 0.6231 & 0.9728 & 0.0000 & 0.2065 & 0.3626 & 0.4475 & 7.5346 \\
& & Processing time & 0.2048 & 0.6240 & -0.3616 & -0.0024 & 0.0406 & 0.1129 & 5.1816 \\
& & Memory & 0.1432 & 0.3112 & -0.2363 & -0.0017 & 0.0318 & 0.1132 & 2.3738 \\
\hline
\end{tabular}

Table 6.14: Statistics of the log2FC from SME-MI to MU-MI in Full-S2

\section{Reasoner Calls}

Figure 6.17 describes the distribution $\log 2 \mathrm{FC}$ for checker calls from using a classical approach to a modularised one for computing a single cut of the MinImps. Note that there is almost no change in behaviour between reasoners and HST implementations. For all situations, most of the observations made $50 \%$ or fewer reasoner calls with the modularised approach, in comparison with the traditional approach. For the cases of type 1 the median and mean are around 5, indicating 32 times fewer reasoner calls, for cases of type 2 the mean is around 4.5 and median close to 4 , so an improvement of 16 times or more happens in roughly $50 \%$ of these instances.

The situation for computing a single MaxNon is very different for that of MinImps. Figure 6.18 shows that cases where modularity help or hinders the number of reasoners calls are both likely. In fact, for cases of type $\mathrm{T} 1$, the $\log 2 \mathrm{FC}$ median is very close to 0 , and the mean is smaller than 1 . As for cases of type 2, both the mean and median are negative, although not smaller than -1 .

Figure 6.19 displays the distribution of fold changes in reasoner calls obtained when going from Basic-MI to Basic-MN. In all situations, the number of reasoner calls stayed about the same or improved. In the cases of type 1, the mean and median are around $\log 2 \mathrm{FC} 4$ and for cases of type 2 around 5. These values indicate that often using Basic-MN makes only $\frac{1}{16}$ of the reasoner calls needed by Basic-MI.

The situation changes entirely in Figure 6.20 where MU-MI(Q) and MU-MI(S) are compared to SME-MN(Q) and SME-MN(S), respectively. This shift is likely a consequence of the fold changes observed in Figures 6.17 and 6.18. In all situations, the mean slightly below 0 and the median around 0.5 . Further, the first and third quartiles are within the interval $[-1,1]$ in all reasoner and case type combinations. Therefore, concerning reasoner calls, SME-MN(Q) and SME-MN(S) are slightly better on a higher proportion of the execution groups, however, when they are worse than $\mathrm{MU}-\mathrm{MI}(\mathrm{Q})$ and $\mathrm{MU}-\mathrm{MI}(\mathrm{S})$ they provoke a sharper depreciation.

\section{Processing Time}

As expected due to the overhead when computing modules, the improvement of modularity in processing time is smaller than in reasoner calls. For MinImps, Figure 6.21 shows that the majority 
of the observations improved in time or stayed roughly the same. In all cases the median is close to 1 , thus around half of executions groups were at least two times faster. There is a high concentration of the observations in the interval $[0,1]$, probably since for some cases, the module overhead is not compensated. Still, cases where MU-MI was four times or faster than Basic-MI for the same HST implementation, are not uncommon (more than 25\%).

Figure 6.22 describes the situation for MaxNons. Interestingly, the fold change shows a better trend on the impact of modularity as the means and medians are all above 0 (even though still below 1). Still, in all situations, roughly $25 \%$ of the groups of executions had worse performance with SME-MN(Q) and SME-MN(S) than with their Basic-MN counterparts. However, as the distribution is well concentrated on the interval $[0,1]$, it is possible to deduce that modularity had an overall positive impact, even if not very significant.

Similarly to the case of reasoner calls, the MaxNon approaches without modularity dominate their MinImp analogues in most instances as it can be inferred from Figure 6.23. In the few cases where Basic-MN was worse than Basic-MI, the difference is not very significant.

In the comparison between modularised approaches (Figure 6.24), the distribution of fold changes is even more concentrated around 0 than for reasoner calls (Figure 6.20). Again, the edge that the MaxNons-based methods had without modularity is lost. There is no reliable evidence to conclude whether MU-MI(Q) and MU-MI(S) are better (or worse) than SME-MN(Q) and SME$\mathrm{MN}(\mathrm{S})$ with respect to processing time.

\section{Memory}

For the impact of modularity when computing a single cut of MinImps, Figure 6.25 shows a trend in memory akin to the results of Full-M2 (Figure 6.13b). There is a high concentration of execution groups that had almost no change, and of groups for which MU-MI(Q) and MU-MI(S) only reached $50 \%$ of the maximum values attained by Basic-MI(Q) and Basic-MI(S), respectively.

The distribution of $\log 2 \mathrm{FC}$ for memory from Basic-MN to SME-MN (Figure 6.26) shows that, for many instances, modularity does not have any significant impact on memory. Nevertheless, the kernel density estimation and centrality measures hint that the impact is, overall, positive more often than not.

When confronting Basic-MI and Basic-MN on memory in Single-M2, the overall trend shows an advantage of the MaxNons-based methods (see Figure 6.27), differently from the fold changes for Full-M1 and Full-M2 that indicated no clear winner.

Next, consider the modularised versions of these methods (MU-MI and SME-MN), whose memory fold changes are described in Figure 6.28. The improvement that Basic-MN had in memory consumption also vanishes when introducing modularity, as it happened with reasoner calls and processing time. The mean of the distribution is close to 0 and the first, second (median) and third quartiles are within -1 and 1 . Although the means lean towards negative values, the median is clearly on the positive interval. In summary, the distribution corroborates on the decision of which method is the best for memory consumption.

\section{Timeouts}

As with experiments Full-M1 and Full-M2, it is also important to analyse the timeout rates of each method in Single-S2. Table 6.15 shows the number of groups of executions per timeout status for each method and implementation.

Table 6.15 displays almost no change is between queue and stack implementations for the same method. SME-MI(Q) and SME-MI(S) are the best methods, followed closely by their MU-MI correspondents (probably due to the increased overhead). After these comes the modularised MaxNons approaches (SME-MN(Q) and SME-MN(S)) and then their non-modularised versions, all these have similar success rates. Finally, the worst method in terms of timeout is Basic-MI. These results agree with the processing time fold changes observed throughout this subsection. 


\begin{tabular}{rrrrrr}
\hline Method & Structure & No timeout & Only timeouts & Some timeout & Success rate \\
\hline Basic-MI & Queue & 1744 & 415 & 432 & $78.42 \%$ \\
& Stack & 1715 & 447 & 461 & $77.11 \%$ \\
\multirow{2}{*}{ SME-MI } & Queue & 2192 & 1 & 2 & $98.56 \%$ \\
& Stack & 2193 & 1 & 1 & $98.60 \%$ \\
\multirow{2}{*}{ MU-MI } & Queue & 2134 & 59 & 60 & $95.95 \%$ \\
\multirow{3}{*}{ Basic-MN } & Stack & 2132 & 62 & 62 & $95.86 \%$ \\
\multirow{3}{*}{ SME-MN } & Queue & 2090 & 98 & 104 & $93.97 \%$ \\
& Stack & 2091 & 97 & 103 & $94.02 \%$ \\
& Queue & 2158 & 49 & 54 & $97.03 \%$ \\
& Stack & 2157 & 50 & 55 & $96.99 \%$ \\
\hline
\end{tabular}

Table 6.15: Number of group of executions per timeout status and method (2224 groups) in Single-M2

Also, as in Full-M1 and Full-M2, timeouts other reasons that prevented successful termination include: stack overflow errors (too many nested recursive calls) and failures in parsing ontologies (e.g. due to unsupported datatypes).

\section{Optimality Rate}

The same methodology to compare methods via optimality rate is applied here. Table 6.16 contains the number of groups of executions, per method and per HST implementation, that where not higher than 1.05 times the minimum value observed for each quantity. Note that each observation counted is a mean for a group of executions with identical parameters and input).

\begin{tabular}{llrrrrrr}
\hline Method & Structure & \multicolumn{2}{c}{ Reasoner calls } & \multicolumn{2}{c}{ CPU time } & \multicolumn{2}{c}{ Memory } \\
& & Groups & \multicolumn{1}{c}{$\%$} & Groups & $\%$ & Groups & $\%$ \\
\hline Basic-MI & Queue & 0 & $0.00 \%$ & 11 & $0.49 \%$ & 38 & $1.71 \%$ \\
& Stack & 0 & $0.00 \%$ & 10 & $0.45 \%$ & 38 & $1.71 \%$ \\
SME-MI & Queue & 81 & $3.64 \%$ & 834 & $37.50 \%$ & 1028 & $46.22 \%$ \\
& Stack & 81 & $3.64 \%$ & 823 & $37.01 \%$ & 1025 & $46.09 \%$ \\
MU-MI & Queue & 404 & $18.17 \%$ & 1348 & $60.61 \%$ & 1495 & $67.22 \%$ \\
& Stack & 340 & $15.29 \%$ & 1255 & $56.43 \%$ & 1394 & $62.68 \%$ \\
Basic-MN & Queue & 1164 & $52.34 \%$ & 625 & $28.10 \%$ & 960 & $43.17 \%$ \\
& Stack & 1166 & $52.43 \%$ & 625 & $28.10 \%$ & 950 & $42.72 \%$ \\
SME-MN & Queue & 740 & $33.27 \%$ & 1299 & $58.41 \%$ & 1388 & $62.41 \%$ \\
& Stack & 740 & $33.27 \%$ & 1303 & $58.59 \%$ & 1366 & $61.42 \%$ \\
\hline
\end{tabular}

Table 6.16: Number and percentage of executions per method that achieve the smallest (best) value in each quantity within a margin of $5 \%$ from the actual minimum in Full-S2

The first immediate observation is that, again, the differences between queue and stack versions are effectively non-existent. Nonetheless, the results for queue implementations are marginally better than for stack, agreeing with the data present in Table 6.13.

As with the optimality rate in Subsection 6.2.2, the Basic-MI computation turns out to be the worst method overall. Interestingly, for reasoner calls, modularity helps MinImps-based approaches but hinders MaxNons-based ones. This difference is probably an undesired effect on the heuristics discussed in Subsection 2.3.4. Still, when analysing processing time and memory spent, the modularised versions are superior to their traditional counterparts, more so in the case of MinImps. For 
both processing time and memory, MU-MI wins over the other methods, followed closely by SMEMN. Then, there is almost a tie between Basic-MN and SME-MI: the first is better on the number of reasoner calls, but the later is better for the other quantities. Finally, there is little difference between queue and stack implementations,

\section{Correlation}

Concerning correlation, Tables 6.17 and 6.18 are analogous to Tables 6.11 and 6.12. As the values do not fluctuate much between the HST implementation (as attested by the similarity of correlation values between the two tables), this aspect is omitted in the discussion that follows.

\begin{tabular}{|c|c|c|c|c|c|c|}
\hline \multirow[t]{2}{*}{ From } & \multirow[t]{2}{*}{ To } & \multirow[t]{2}{*}{$\mathrm{N}$} & \multirow[t]{2}{*}{ Quantity } & \multicolumn{3}{|c|}{ Correlation (Kendall's $\tau$ ) } \\
\hline & & & & $\begin{array}{l}\text { Ontology size } \\
\text { (axioms) }\end{array}$ & $\begin{array}{l}\text { Average for } \\
\text { "from" }\end{array}$ & $\begin{array}{l}\text { Relative module } \\
\text { size }\end{array}$ \\
\hline \multirow[t]{3}{*}{ Basic-MI } & SME-MI & 1742 & Reasoner calls & 0.3513 & 0.6343 & -0.1832 \\
\hline & & & CPU time & 0.5141 & 0.8229 & -0.1459 \\
\hline & & & Memory & 0.4866 & 0.7142 & -0.2076 \\
\hline \multirow[t]{3}{*}{ Basic-MI } & MU-MI & 1744 & Reasoner calls & 0.3378 & 0.7306 & -0.1302 \\
\hline & & & CPU time & 0.4934 & 0.8486 & -0.1191 \\
\hline & & & Memory & 0.4813 & 0.7971 & -0.1544 \\
\hline \multirow[t]{3}{*}{ Basic-MN } & SME-MN & 2066 & Reasoner calls & 0.0350 & 0.4834 & -0.2244 \\
\hline & & & CPU time & 0.2862 & 0.4783 & -0.2642 \\
\hline & & & Memory & 0.2459 & 0.4544 & -0.2724 \\
\hline \multirow[t]{3}{*}{ Basic-MI } & Basic-MN & 1743 & Reasoner calls & 0.2493 & 0.4455 & -0.0376 \\
\hline & & & CPU time & 0.4294 & 0.6921 & -0.0876 \\
\hline & & & Memory & 0.3590 & 0.5395 & -0.1208 \\
\hline \multirow[t]{3}{*}{ MU-MI } & SME-MN & 2138 & Reasoner calls & -0.0437 & -0.1862 & -0.3645 \\
\hline & & & CPU time & -0.0078 & -0.1154 & -0.3351 \\
\hline & & & Memory & 0.0297 & -0.0380 & -0.3620 \\
\hline
\end{tabular}

Table 6.17: Correlations between log2FC between methods using queue as HST structure and ontology size, average value of the quantities measured and the relative size of the module for Single-M2 (values in grey had p-value $>0.01$ )

The association is direct strong between the magnitude of the average values for quantities measured for Basic-MI and the fold changes when using MU-MI. The same occurs from Basic-MI to SME-MI for processing time and memory (for reasoner calls the association of reasoner calls was only moderate).

Aside these, there is a moderate positive association between the fold change of processing time from Basic-MI to SME-MI and the size of the ontology. Also, there is a direct, but moderate, correlation between the time and memory spent by Basic-MI and the fold change when switching to Basic-MN. This latter association vanishes in the modularised versions, reflecting the results seen previously in this subsection.

The remaining correlations are either mild or weak, therefore cannot help in identifying the potential causes differences in performance between the methods considered.

\subsection{Discussion}

According to the experiments performed, modularity almost always improves the computation MinImps and MaxNons, especially when the whole sets are needed. This improvement is more evident in reasoner calls, but also significant in memory consumed and processing time spent. The 


\begin{tabular}{|c|c|c|c|c|c|c|}
\hline \multirow[t]{2}{*}{ From } & \multirow[t]{2}{*}{ To } & \multirow[t]{2}{*}{$\mathrm{N}$} & \multirow[t]{2}{*}{ Quantity } & \multicolumn{3}{|c|}{ Correlation (Kendall's $\tau$ ) } \\
\hline & & & & $\begin{array}{c}\text { Ontology size } \\
\text { (axioms) }\end{array}$ & $\begin{array}{l}\text { Average for } \\
\text { "from" }\end{array}$ & $\begin{array}{c}\text { Relative module } \\
\text { size }\end{array}$ \\
\hline \multirow[t]{3}{*}{ Basic-MI } & SME-MI & 1713 & Reasoner calls & 0.3512 & 0.6558 & -0.1798 \\
\hline & & & CPU time & 0.5112 & 0.8266 & -0.1568 \\
\hline & & & Memory & 0.4896 & 0.7314 & -0.2051 \\
\hline \multirow[t]{3}{*}{ Basic-MI } & MUMI & 1715 & Reasoner calls & 0.3441 & 0.7163 & -0.1571 \\
\hline & & & CPU time & 0.4971 & 0.8448 & -0.1398 \\
\hline & & & Memory & 0.4914 & 0.7937 & -0.1756 \\
\hline \multirow[t]{3}{*}{ Basic-MN } & SME-MN & 2067 & Reasoner calls & 0.0355 & 0.4833 & -0.2243 \\
\hline & & & CPU time & 0.2852 & 0.4738 & -0.2619 \\
\hline & & & Memory & 0.2433 & 0.4560 & -0.2705 \\
\hline \multirow[t]{3}{*}{ Basic-MI } & Basic-MN & 1714 & Reasoner calls & 0.2418 & 0.4419 & -0.0450 \\
\hline & & & CPU time & 0.4268 & 0.6863 & -0.1014 \\
\hline & & & Memory & 0.3580 & 0.5451 & -0.1238 \\
\hline \multirow[t]{3}{*}{ MU-MI } & SME-MN & 2138 & Reasoner calls & -0.0393 & -0.0939 & -0.3002 \\
\hline & & & CPU time & 0.0071 & -0.0554 & -0.2822 \\
\hline & & & Memory & 0.0261 & 0.0187 & -0.3036 \\
\hline
\end{tabular}

Table 6.18: Correlations between the fold changes and ontology size, average value of the quantities measured and the relative size of the module for Single-M2 (values in grey had p-value $>0.01$ ).

effects could be seen in different environments, with two distinct reasoners and with two types of test inputs in a decently sized corpus.

Also, for the computation of the whole set, the modularised strategies based on MinImps is generally superior to the other methods. For the computation of only a single solution, MU-MI and SME-MN approaches are tied in performance: some cases are much better with one than the other. Moreover, in all quantities evaluated, many instances had similar performance with both strategies. Interestingly, Basic-MN dominates MU-MI in every aspect. Still, the difference vanishes after modularisation.

Furthermore, the magnitude of the quantities (reasoner calls, memory and time) was often directly strongly related to the gains obtained with modularity. Although correlation does not imply causation, this is good evidence that modularity becomes more worthwhile as the costs without it increase, showing that the overhead is compensated more easily in harder instances.

Only occasionally the number of axioms of an ontology was directly related to gains via modularity. Also, only mild and weak correlations occur between the relative size of a module and the fold changes observed. In this latter case, the trend was of negative association which conforms (although it does not fully support) to the intuition that smaller modules produce better gains.

On a smaller note, when computing a single solution, almost no difference was observed between queue and stack implementations for MinImps-based methods. This similarity in behaviour occurs in fold changes, timeouts and correlations.

Note that the results here, as discussed earlier, are bound to the distribution modules, which are generally very small for all inputs. For different data (either in type or with larger signatures), the modules could be bigger, and thus, the results obtained here would not generalise. Still, as evidence from existing studies concerning LBMs modules are usually small in practice [SSZ09, SQJH08, Moo10, Hor11]. Hence modularity can still be considered a worthwhile optimisation. 

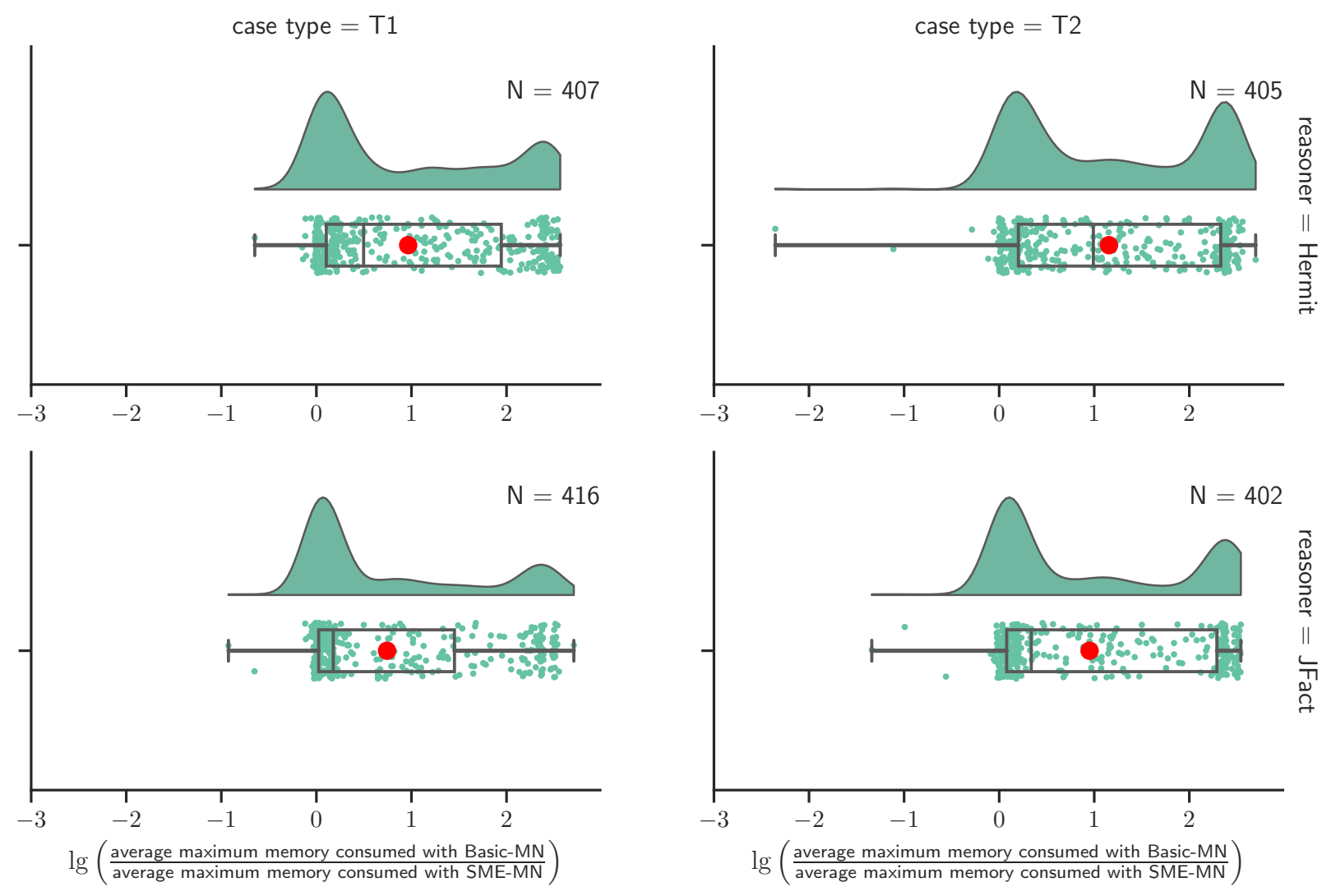

(a) Raincloud plot for Full-M1
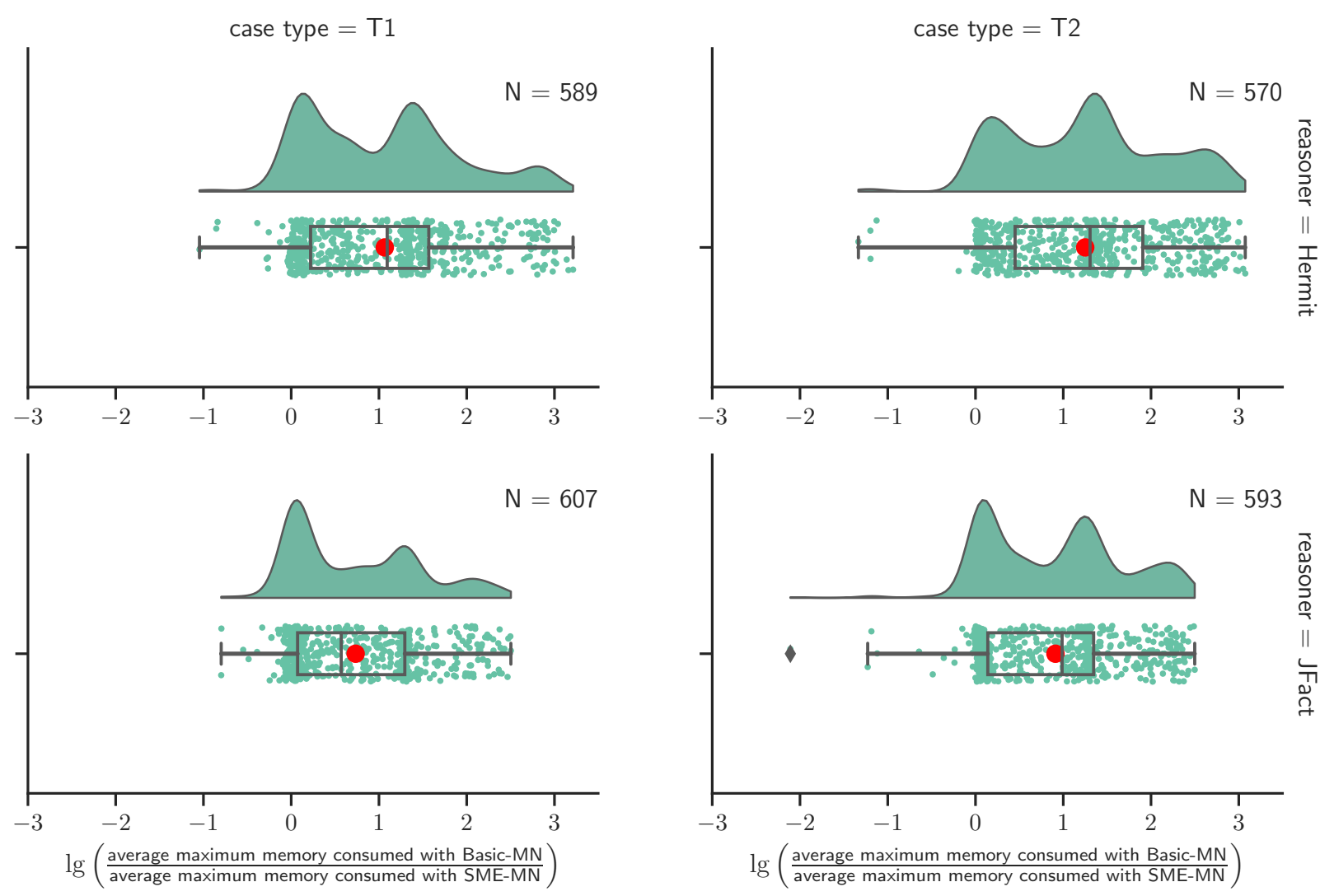

(b) Raincloud plot for Full-M2

Figure 6.14: Distribution of maximum memory (VmHWM) consumed in average by Basic-MN and SME$M N$ 

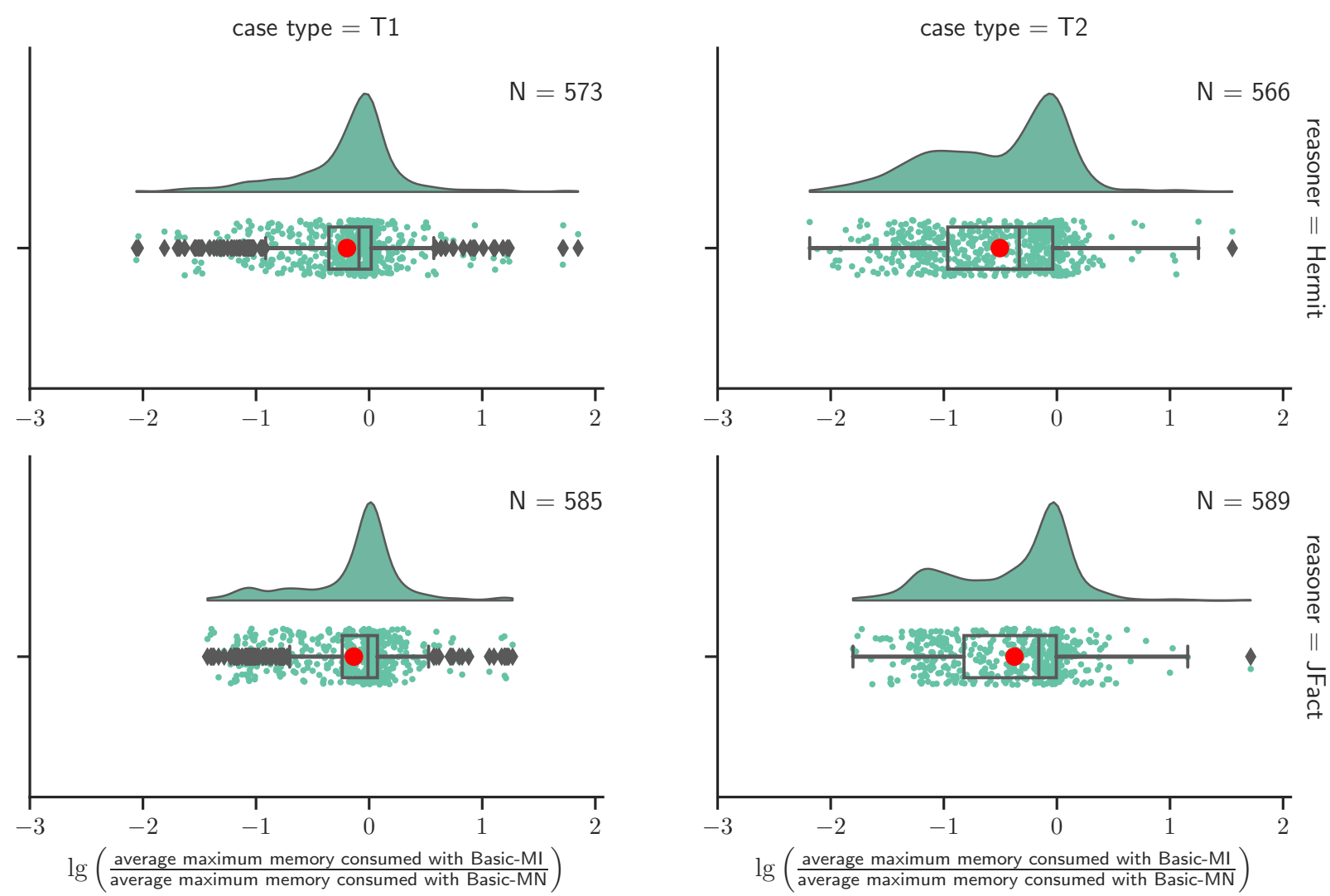

Figure 6.15: Distribution of maximum memory (VmHWM) consumed in average by Basic-MI and BasicMN in Full-M2
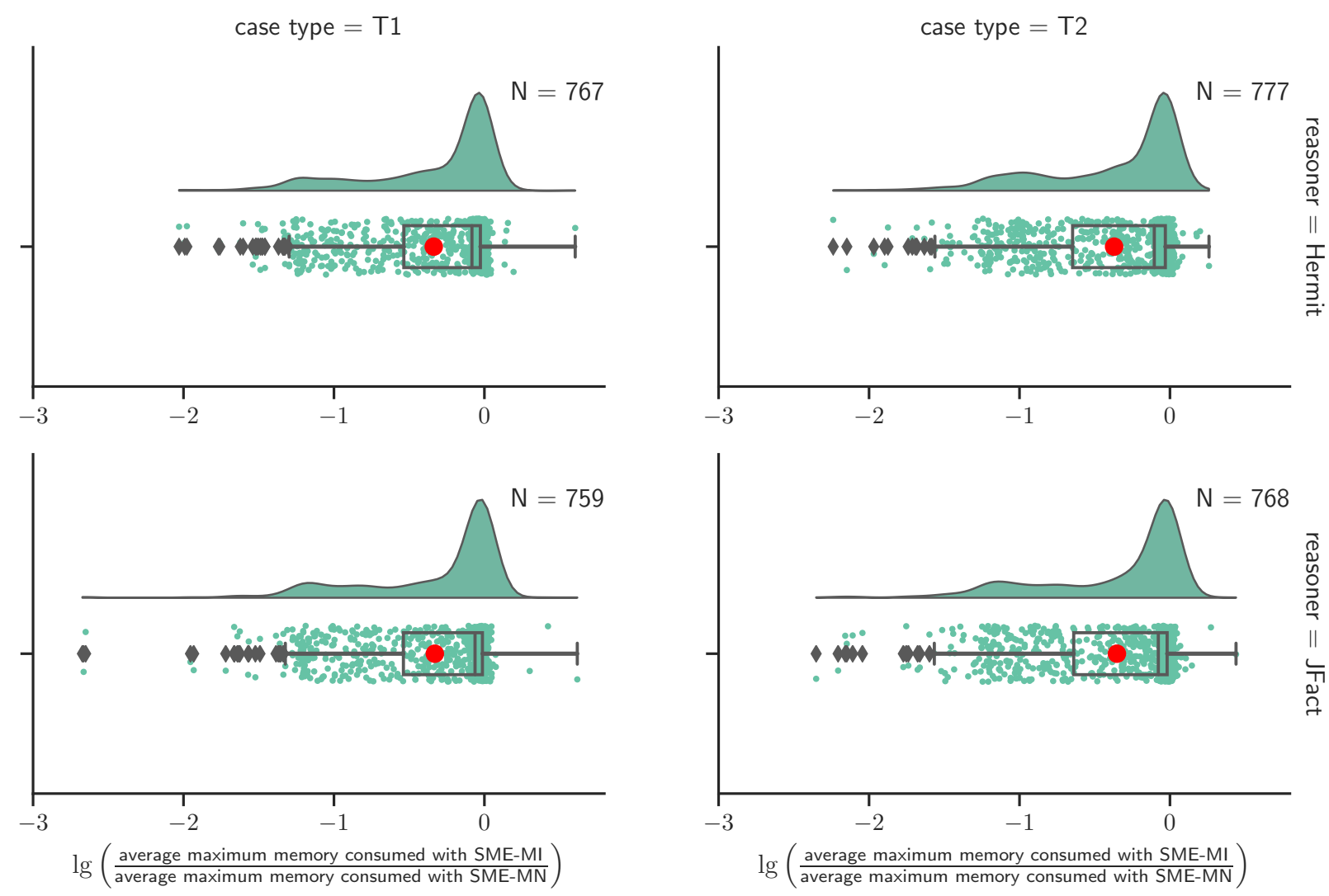

Figure 6.16: Distribution of maximum memory (VmHWM) consumed in average by SME-MI and SME-MN in Full-M2 

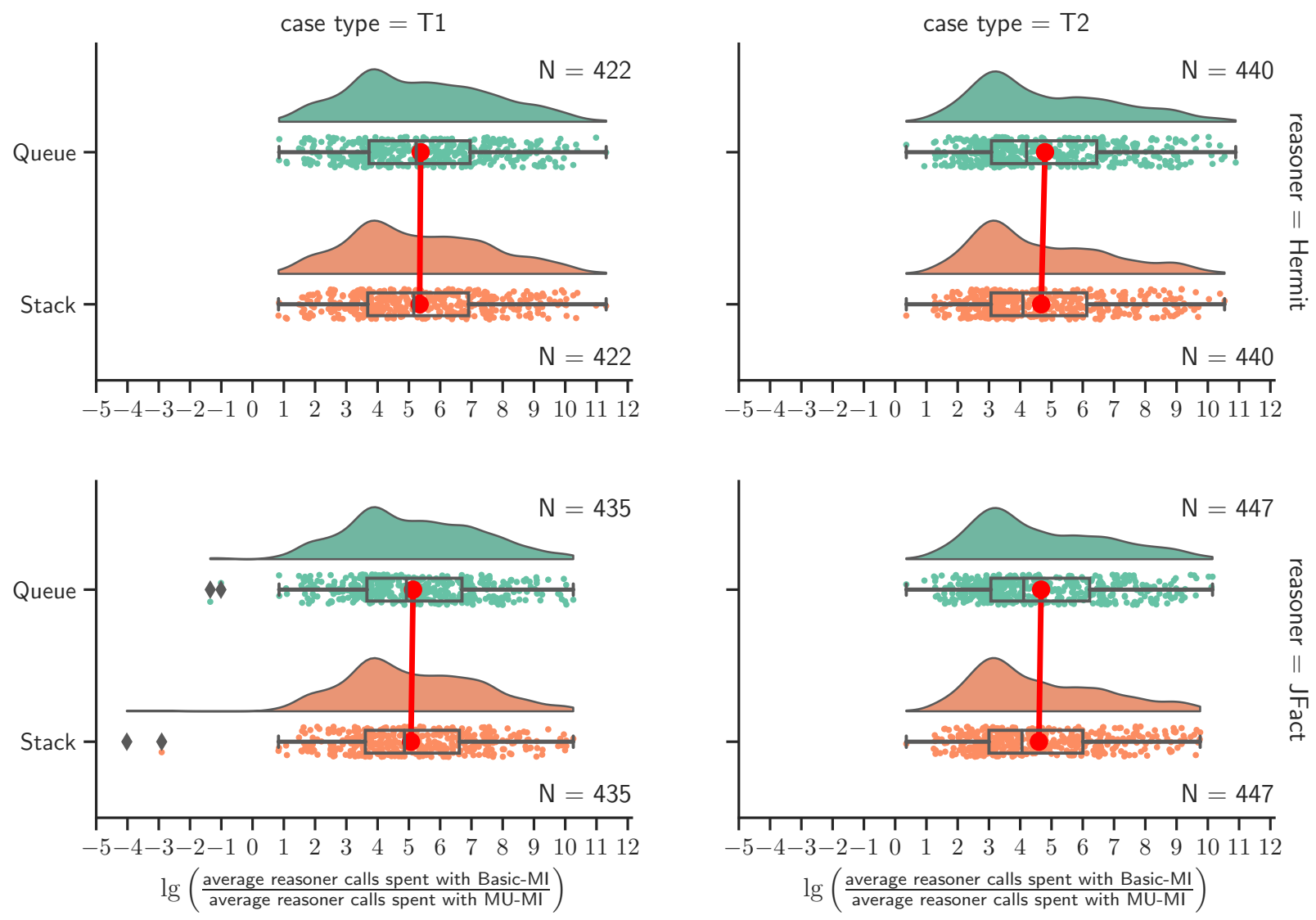

Figure 6.17: Distribution of log2FC for reasoner calls spent in average by Basic-MI and MU-MI in SingleM2 


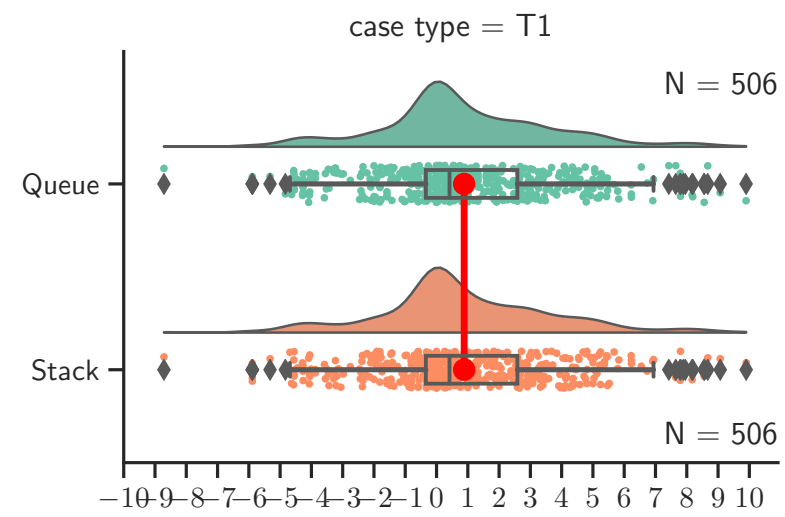

case type $=\mathrm{T} 2$
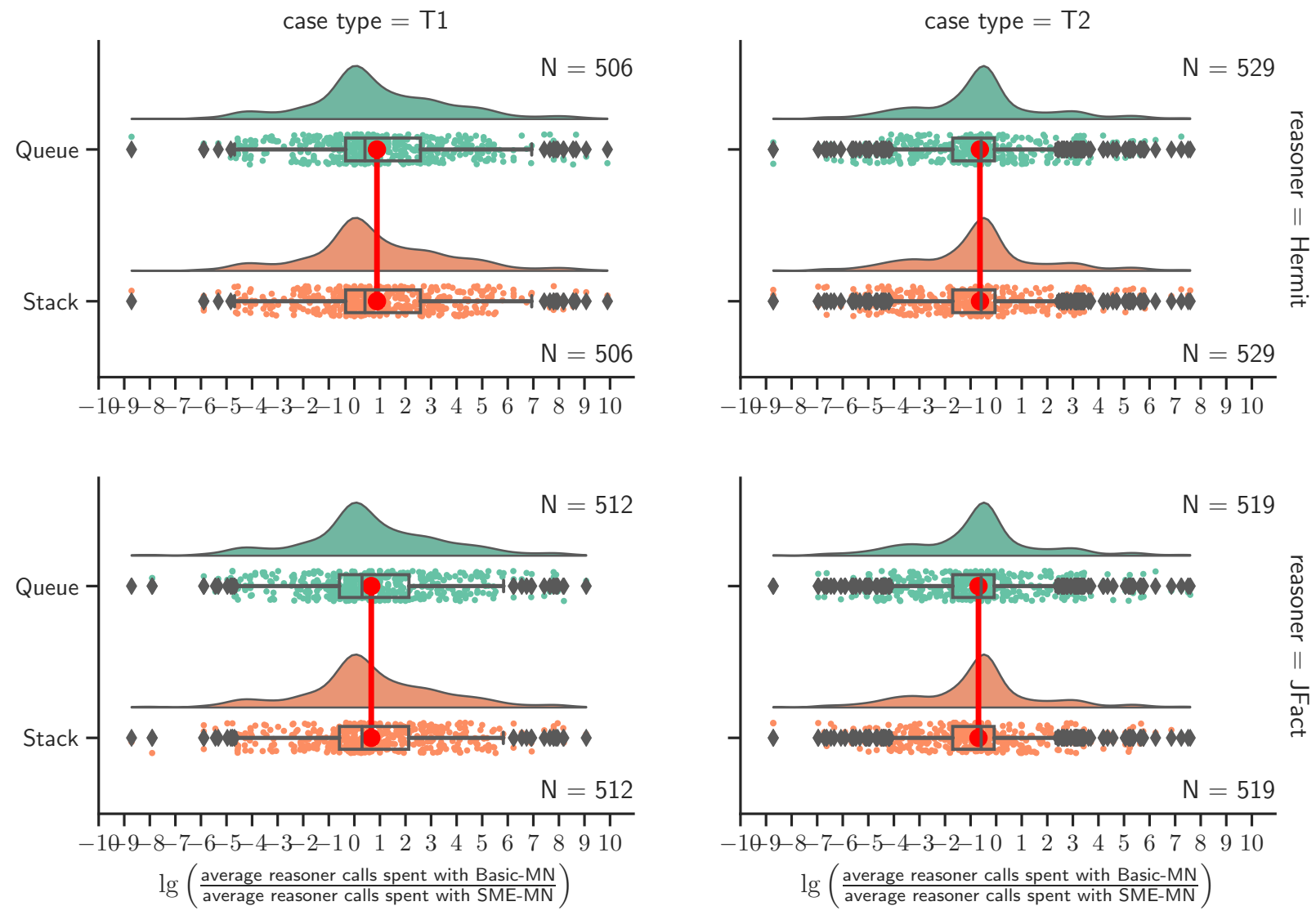

Figure 6.18: Distribution of log2FC for reasoner calls spent in average by Basic-MN and SME-MN in Single-M2 

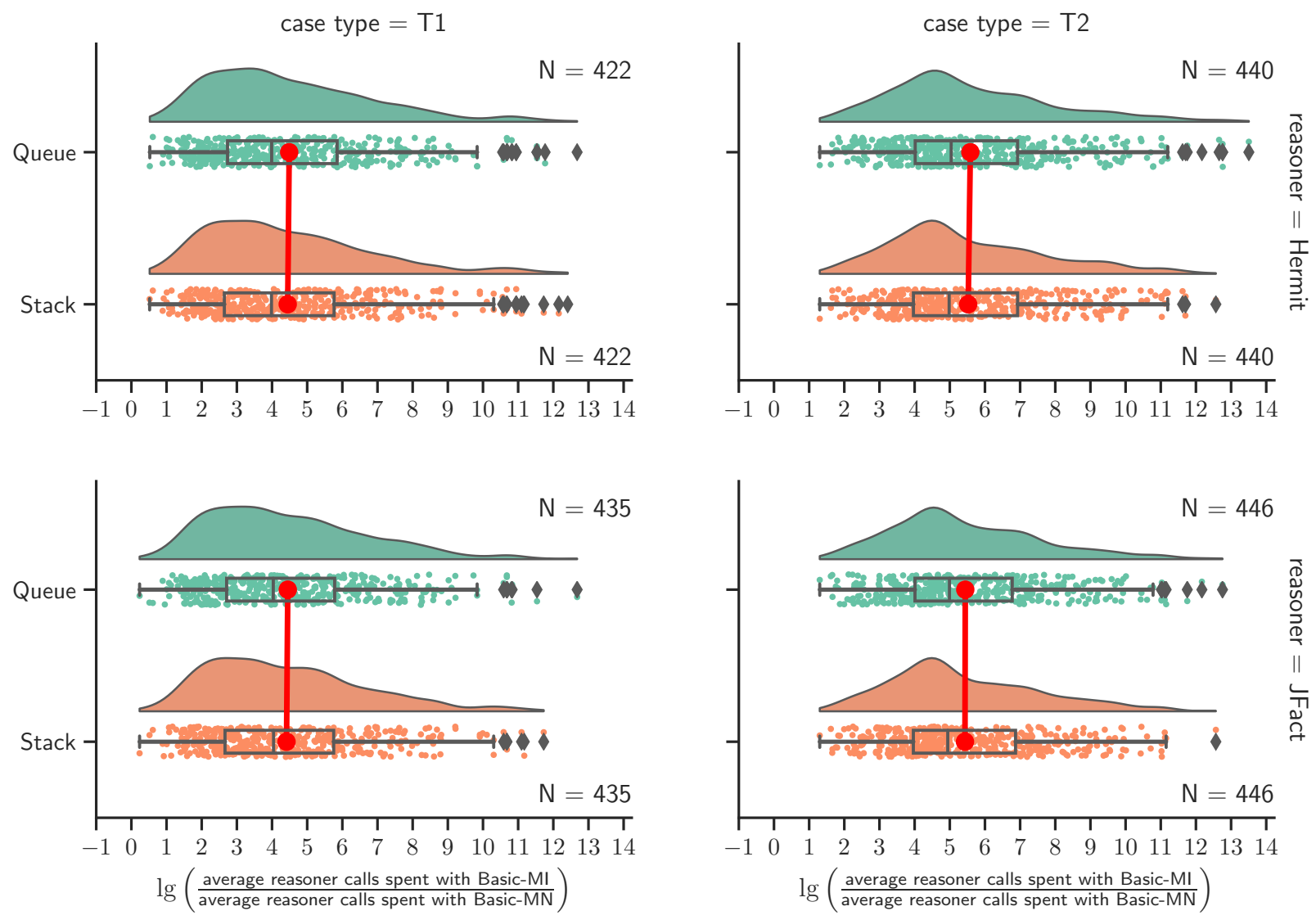

Figure 6.19: Distribution of log2FC for reasoner calls spent in average by Basic-MI and Basic-MN in Single-M2 

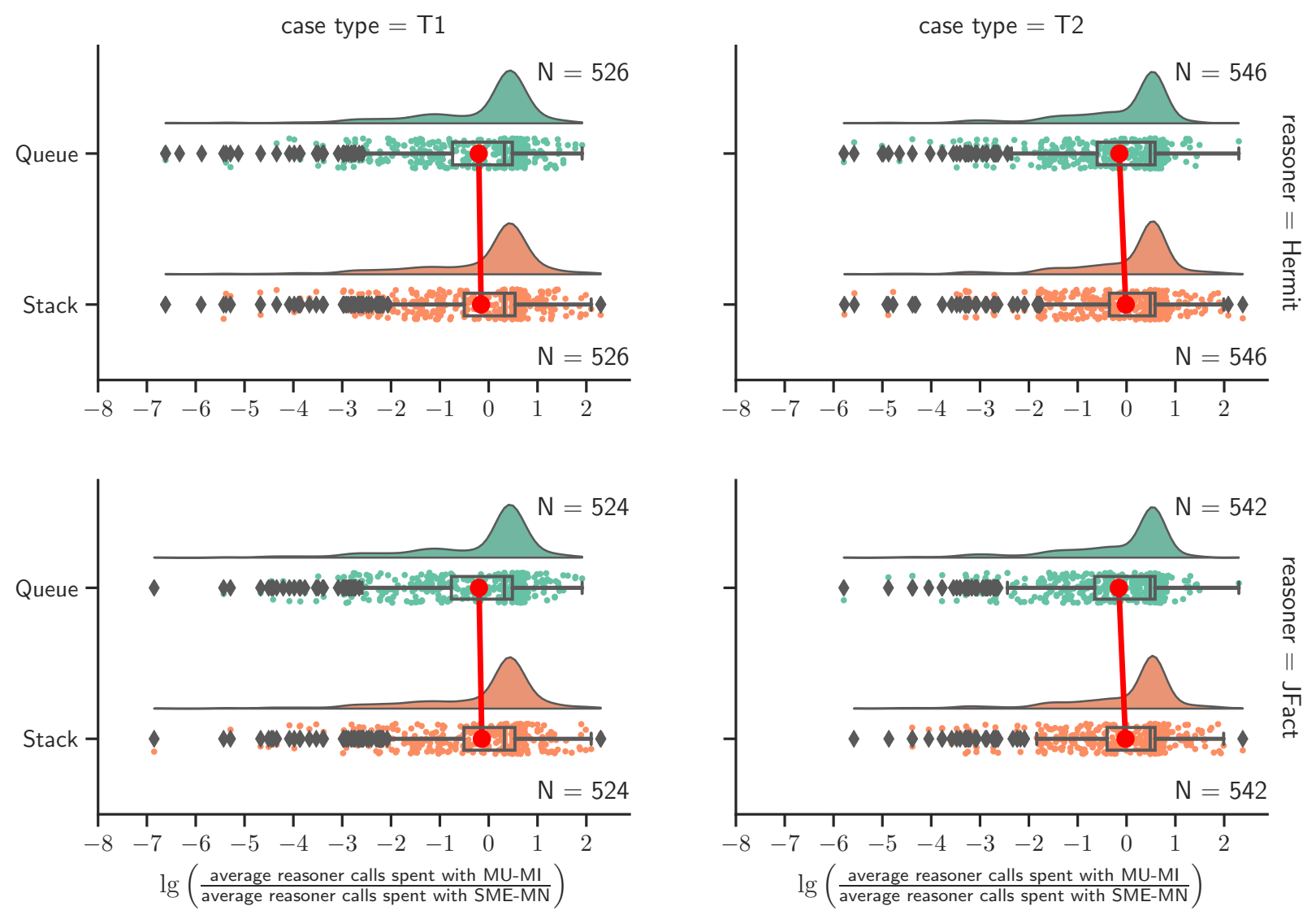

Figure 6.20: Distribution of log2FC for reasoner calls spent in average by MU-MI and SME-MN in SingleM2 

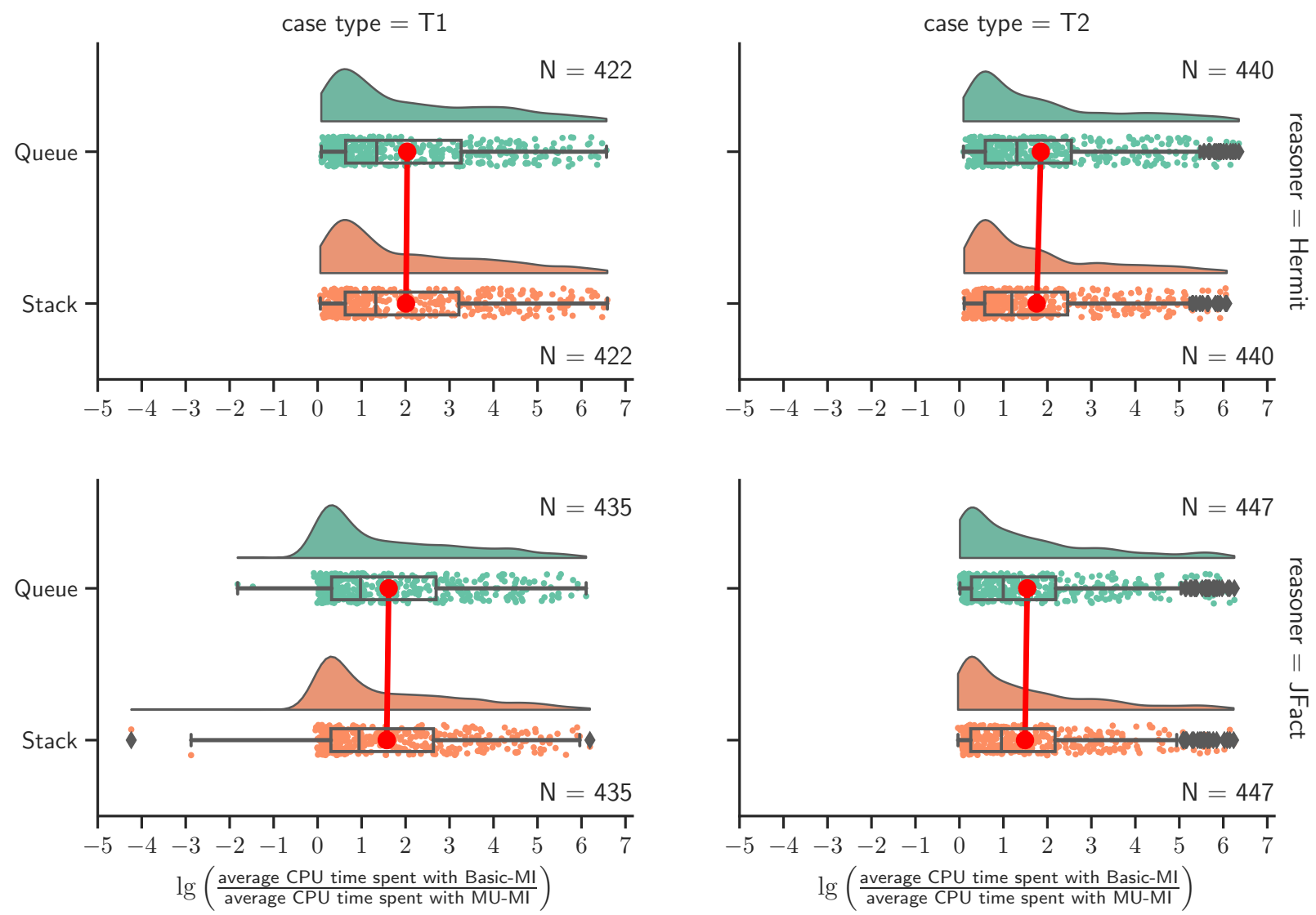

Figure 6.21: Distribution of log2FC for CPU time spent in average by Basic-MI and MU-MI in Single-M2 

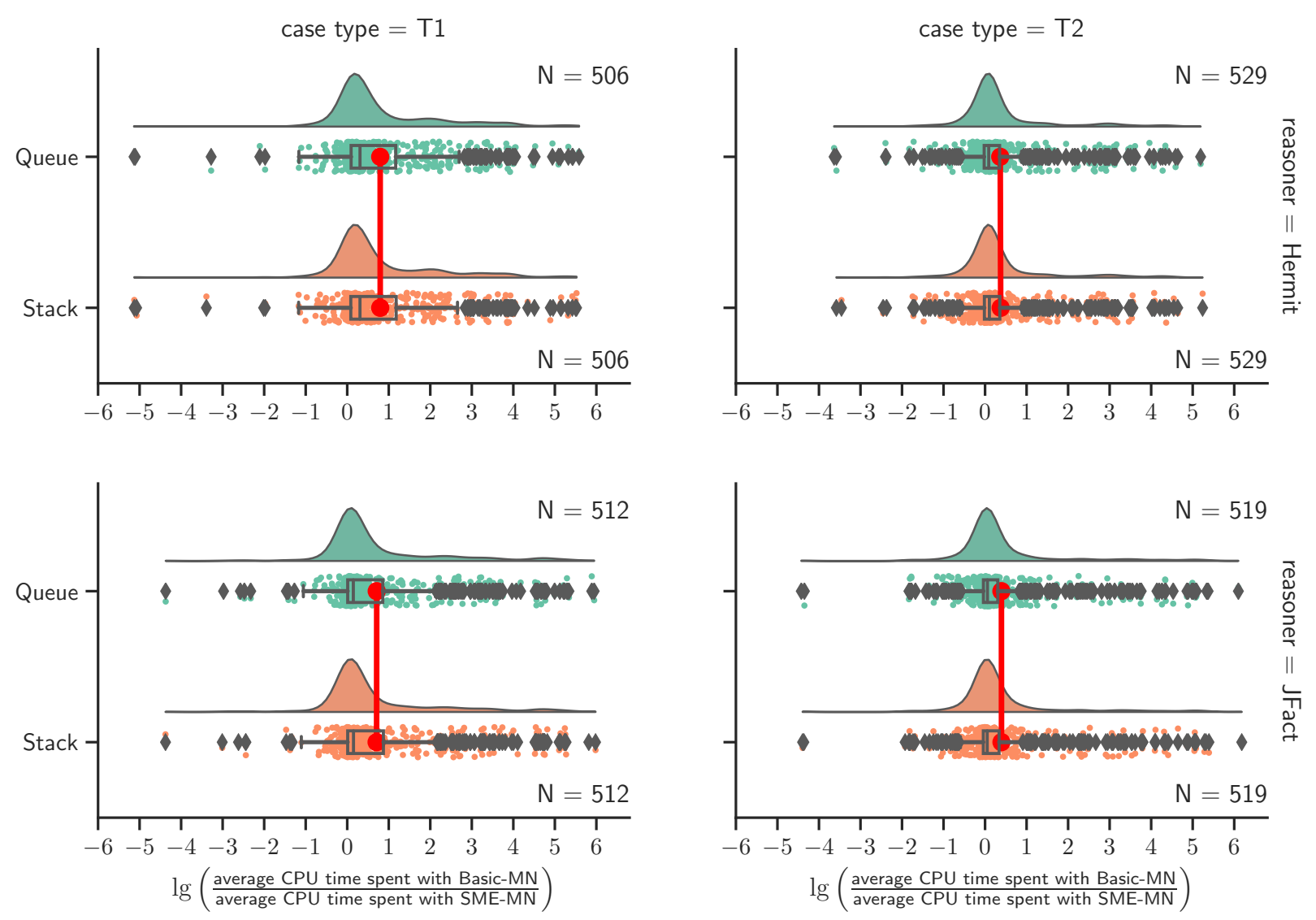

Figure 6.22: Distribution of log2FC for $C P U$ time spent in average by Basic-MN and SME-MN in SingleM2 

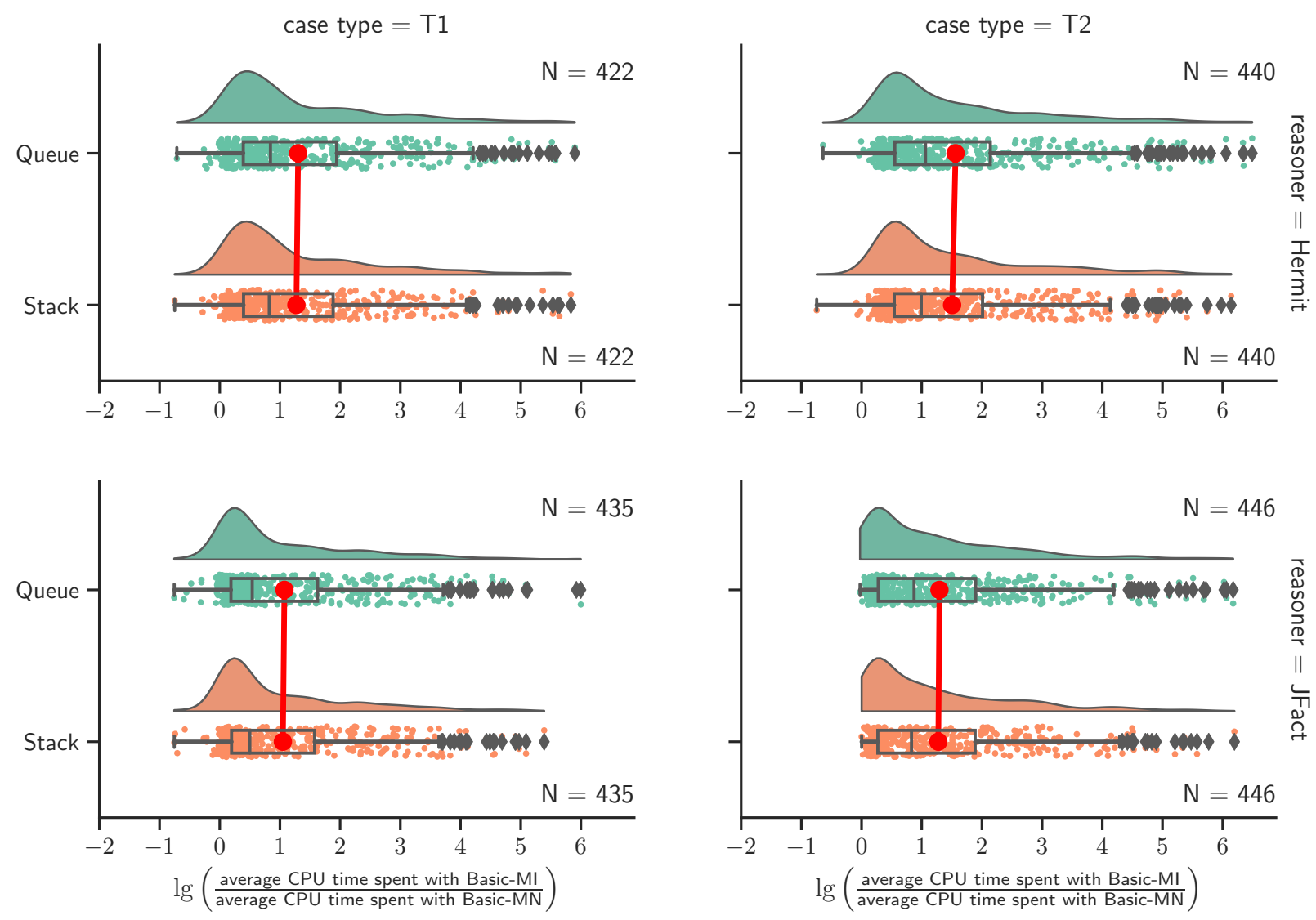

Figure 6.23: Distribution of log2FC for CPU time spent in average by Basic-MI and Basic-MN in Single-M2
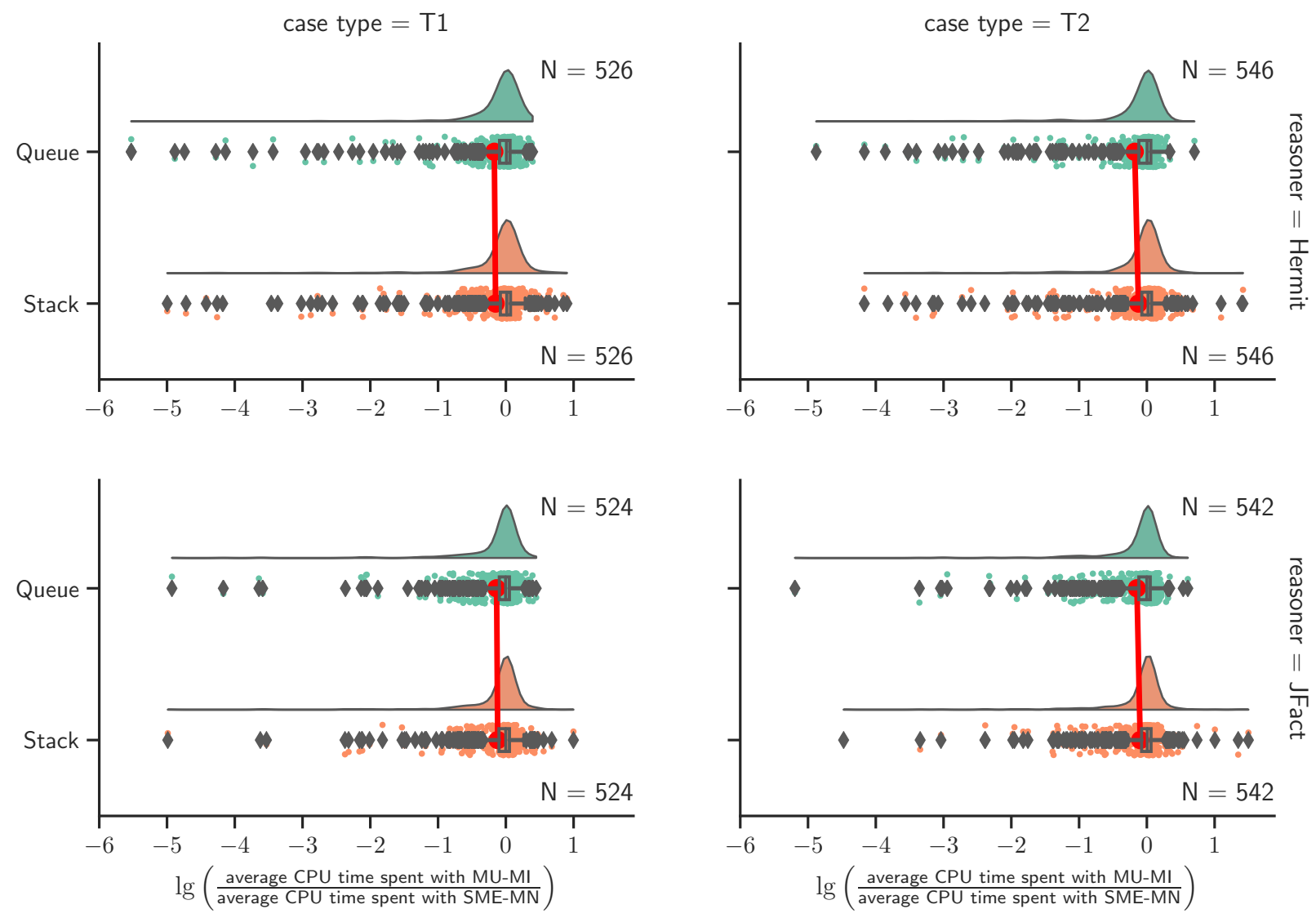

Figure 6.24: Distribution of log2FC for CPU time spent in average by $M U-M I$ and SME-MN in Single-M2 

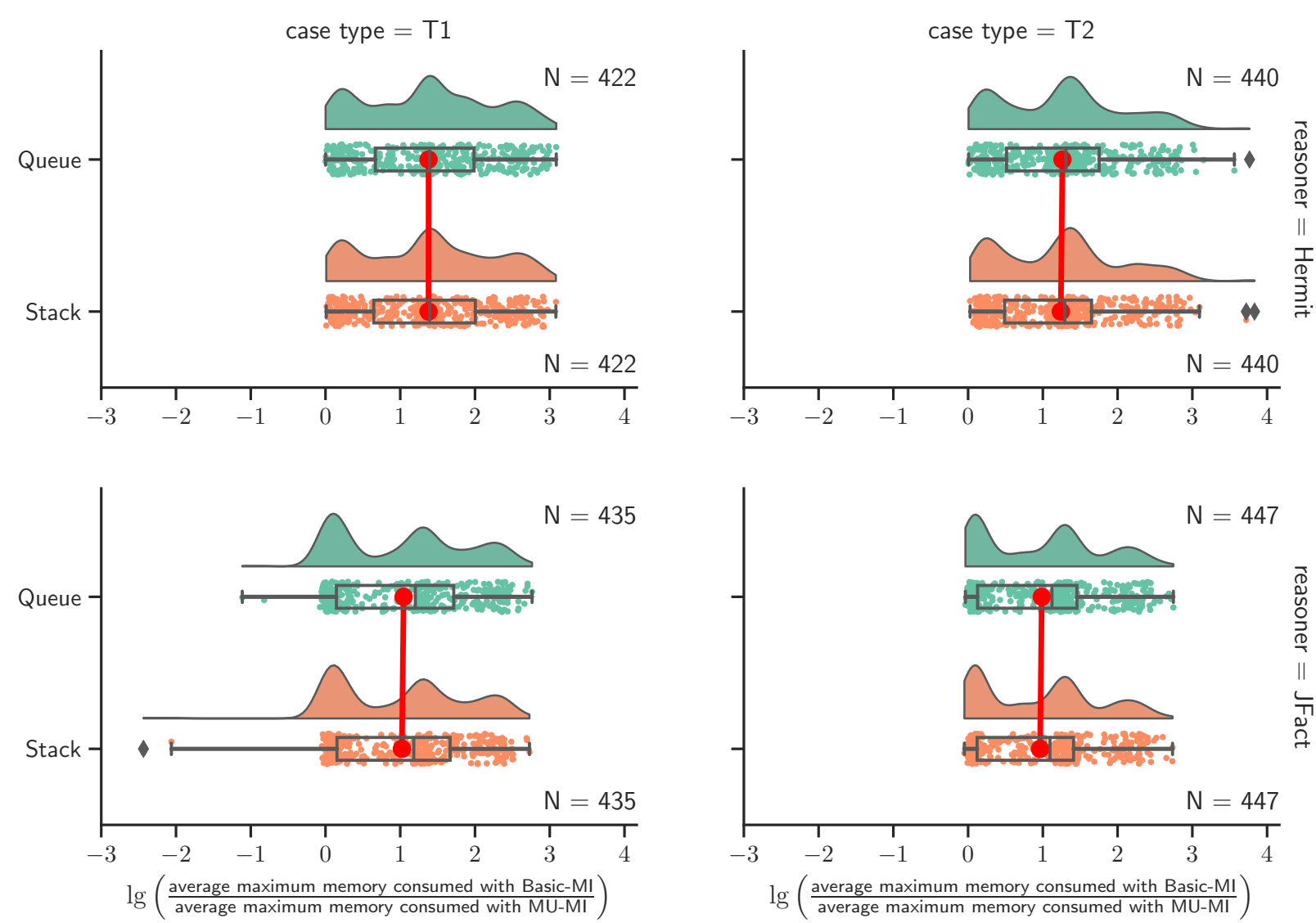

Figure 6.25: Distribution of maximum memory (VmHWM) consumed in average by Basic-MI and MU-MI in Single-M2 

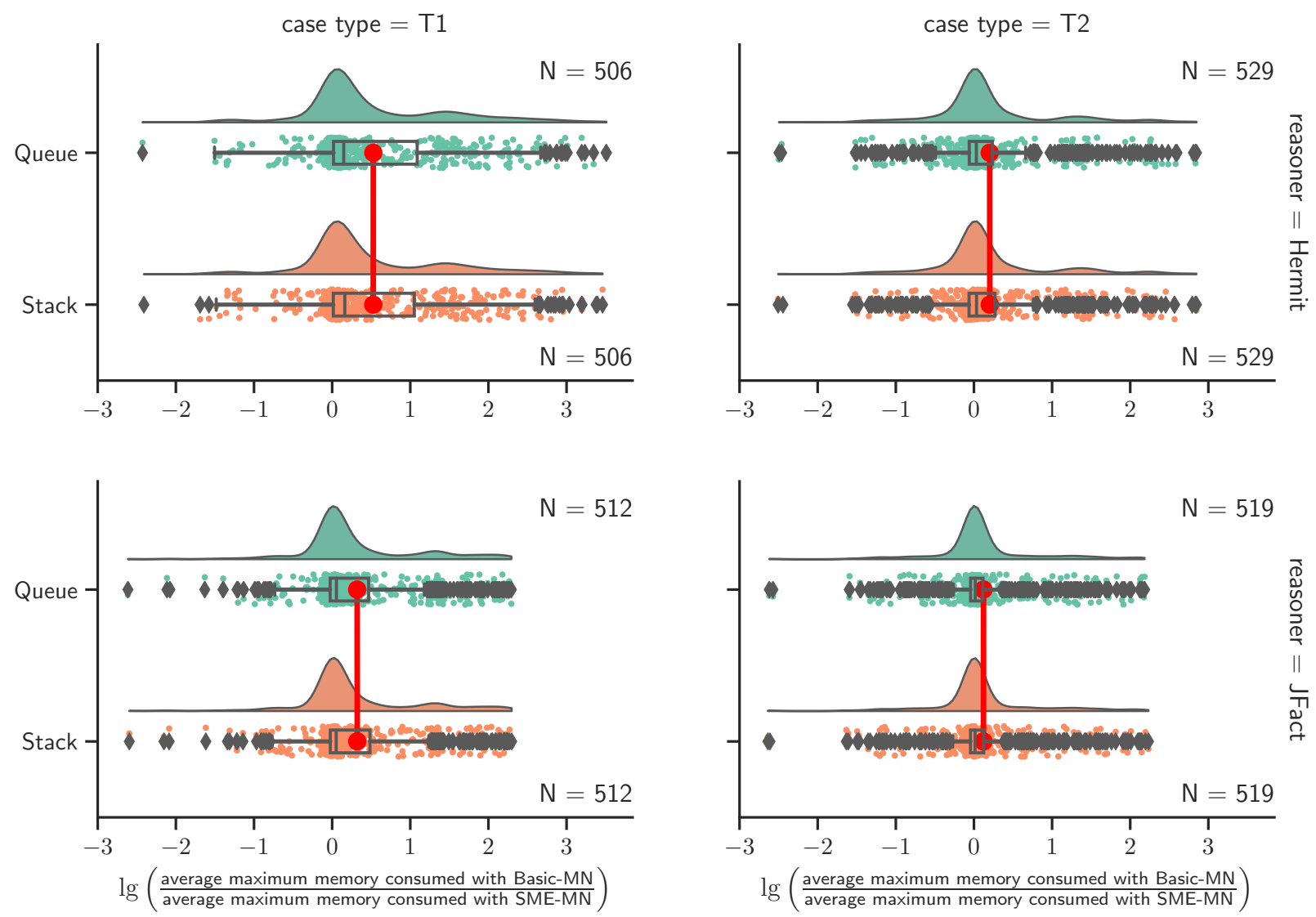

Figure 6.26: Distribution of maximum memory (VmHWM) consumed in average by Basic-MN and SME$M N$ in Single-M2 



Figure 6.27: Distribution of maximum memory (VmHWM) consumed in average by Basic-MI and Basic$M N$ in Single-M2 

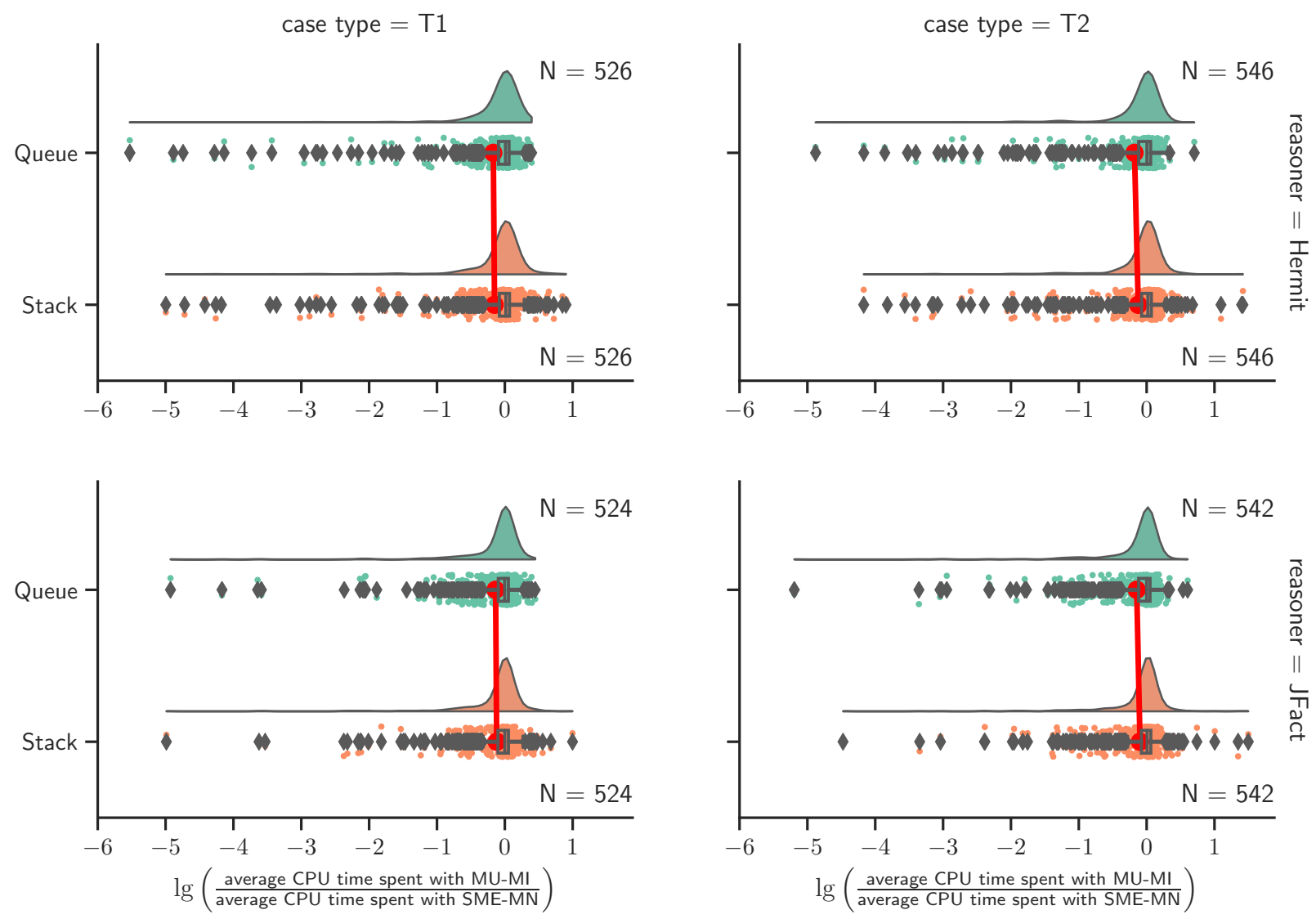

Figure 6.28: Distribution of maximum memory (VmHWM) consumed in average by $M U-M I$ and $S M E-M N$ in Single-M2 


\section{Chapter 7}

\section{Modularity-based Stratification}

The third research question introduced in Section 3.3 concerns is the multiple outcome problem. The size of MinImps and MaxNons is potentially exponential on the number of axioms in an ontology, and each of their elements can be as large as the whole ontology itself. Hence, manually performing the role of incision functions or selection functions can be unfeasible.

In Belief Change, this problem is usually addressed via orderings on the outcomes. The seminal work by Alchourrón, Gärdenfors and Makinson [AGM85] already includes an approach of this nature. The postulates of the classical AGM theory are related to relationships on the potential outcomes, which satisfy specific properties. Further, the AGM postulates and constructions are also equivalent to operations defined via orderings of formulas called Epistemic Entrenchments [Gär88]. Among similar proposals are Generalized Epistemic Entrenchments [Rot92], Ensconscement [Wil94] and Epistemic Relevance [Neb91] which vary on which restrictions they impose on such orderings and whether the apply to belief sets or belief bases.

Issues of the same nature occur in other areas such as inconsistency-tolerant reasoning, where stratification-based solutions have been proposed $\left[\mathrm{BCD}^{+}\right.$93, QP07], similar techniques have also been developed for default reasoning [BH95]. While stratifications do not necessarily represent linear orders, they are easier to obtain as they do not require a distinction between each pair of axioms. Hence, while ties may still occur in such approaches, a user may have to select between a fewer number of "best candidates" instead of choosing among all possible ones.

\subsection{Stratification for Belief Change in Description Logics}

Cóbe [Cób14] discusses some existing approaches to ontology stratification procedures to solve the problem of Ontology Merging. In Ontology Merging, the objective is to obtain a single ontology from a given set of ontologies, with consistency as a requirement. Since merging operations are closely related to Belief Change, these methods may also be applied to diminish or even solve the problem of multiple plausible outcomes in Ontology Repair.

The techniques presented by Cóbe [Cób14] do not use any extralogical feature (e.g. provenance), what aligns with this thesis' objectives. In particular, most of them rely on the syntax of axioms and reasoning alone. Some works, assume the existence of a convenient relationship and do not mention its source, while others assume it as an input given by the user [FUV83, Gär88, Rot92]. Even these studies still provide interesting guidelines concerning minimal change and relevance and are, in general, well equipped with theoretical results.

Notably, the generality or specificity of axioms can serve as a criterion to determine when they are more or less important (although there is not a consensus on which of them). Such a metric has been used in inconsistent tolerant reasoning [QP07], even though it lacks a commonly agreed definition for complex axioms, specially those that are not easily converted from OWL 2 to concept inclusions in DLs (as in Table 2.2).

Also, few studies use modularity sense [GW17, GSW18]. Guimarães and Wassermann propose a stratification method using the $T \perp^{*}$-LBMs [GW17], while Guimarães, Sattler and Wassermann 
investigate how $T$ and $\perp$-LBMs behave with the same method [GSW18]. Since both stdudies are part of this thesis, many of their results and definitions will be reproduced in this chapter along with some new results.

\subsection{Stratification Methods}

Before detailing the methods evaluated, this section introduces some fundamental concepts and establish conventions used throughout this chapter.

This thesis views ontologies as finite sets of formulas (finite belief bases) in a description logic. Further, the approach adopted here is the same employed by Benferhat et al. [BBPW17], but without the distinction between ABox and TBox axioms. Henceforth, a stratified ontology $\mathcal{O}$ to be a partitioned set of axioms: $\mathcal{O}=\left\{\mathcal{O}_{i}\right\}_{0 \leq i \leq n}$ (with $\mathcal{O}_{i} \cap \mathcal{O}_{j}=\emptyset$, if $i \neq j$ ). By convention, the most important axioms are those at the strata with the lowest index, that is, if $i<j$, then the axioms in $\mathcal{O}_{i}$ are more important than (or preferred over) the axioms in $\mathcal{O}_{j}$. Moreover, if an axiom $\alpha$ is at the stratum $\mathcal{O}_{i}$, than $\alpha$ has rank $i(\operatorname{rank}(\alpha)=i)$. Through this thesis the notions of ranks and partitions are used interchangeably.

The stratification approaches detailed in this section are induced by a partial order of the axioms. Formally, if $S$ is a set and $\prec$ a partial order on $S$ then, the stratification of $S$ induced by $\prec$ is such that, for each $a \in S$ :

$$
\operatorname{rank}(a)=\left\{\begin{array}{lr}
0, & \text { if } a \text { is minimal w.r.t. } \prec \\
1+\max _{\{b \in S \mid b \prec a\}} \operatorname{rank}(b), & \text { otherwise }
\end{array}\right.
$$

\subsubsection{Axiom Specificity}

Axiom specificity (AxS) was described by Qi and Pan [QP07], based on the work of Benferhat, Baida and Cuppens [BBC03] that used this notion of importance in access-control applications. A similar notion was applied in default logic terminologies [BH95]. This criterion considers the most specific axioms as the most important ones. According to the definition in [QP07] $\alpha_{1}=\mathrm{C}_{1} \sqsubseteq \mathrm{D}_{1}$ is more specific than $\alpha_{2}=\mathrm{C}_{2} \sqsubseteq \mathrm{D}_{2}$ (written $\alpha_{1} \prec$ AxS $\alpha_{2}$ ) in $\mathcal{O}$ if $\mathcal{O} \vDash \mathrm{C}_{1} \sqsubseteq \mathrm{C}_{2}$, but $\mathcal{O} \not \mathrm{C}_{2} \sqsubseteq \mathrm{C}_{1}$. Therefore, AxS is the stratification of axioms induced by $\prec_{\text {AxS }}$.

This thesis extends the definition of specificity $\left(\prec_{\mathrm{AxS}}\right)$ due to Qi and Pan [QP07] to other types of axioms, such as role (chain) inclusions, using an analogous methodology for comparison (e.g. check inclusion between the left-hand sides of two axioms). The axioms are divided into five categories: CBox axioms, which associate concepts; ABox axioms which associate individuals to concepts or roles, RBox axioms, representing those that relate roles and DBox axioms which involve datatypes and PBox which contain data properties. ABox axioms (concept and role assertions) are minimal axioms, as suggested by Cóbe [Cób14]. That is, given an ontology $\mathcal{O}$, if $\alpha$ is a class or role assertion, then $\alpha$ is minimal w.r.t. $\prec_{\mathrm{AxS}}$. Excluding relations with ABox axioms, axioms from different categories are incomparable.

This method is syntax dependent, i.e., two ontologies $\mathcal{O}_{1}$ and $\mathcal{O}_{2}$ can have equivalent axioms (for every $\alpha_{1} \in \mathcal{O}_{1}$ there exists $\alpha_{2} \in \mathcal{O}_{2}$, such that $\alpha_{1} \equiv \alpha_{2}$, and vice versa), and yet have distinct stratifications. It is possible to change the rank of an axiom by rewriting it in another way. Consider the axiom $\mathrm{A} \sqcap \exists \mathrm{r} . \mathrm{B} \sqsubseteq \mathrm{X}$, a simple absorption, a common optimization in reasoners, would rewrite it equivalently as $\mathrm{A} \sqsubseteq \forall \mathrm{r} . \neg \mathrm{B} \sqcup \mathrm{X}$ that is (usually) less specific than its original version. Moreover, this criterion ignores the right-hand side of axioms. Hence in the ontology $\mathcal{O}=\left\{\mathrm{A} \sqsubseteq \mathrm{C}_{1}, \mathrm{~B} \sqsubseteq \mathrm{C}_{2}, \mathrm{C}_{1} \sqsubseteq \mathrm{C}_{2}\right\}$, the axioms $\mathrm{A} \sqsubseteq \mathrm{C}_{1}$ and $\mathrm{B} \sqsubseteq \mathrm{C}_{2}$ would be placed in the same stratum, even though we could say that the first one is more specific than the because since $A$ and $C_{1}$ are subconcepts of $C_{2}$ [Cób14].

The dependency on classification to compute AxS also interferes with their application on inconsistent ontologies, as the hierarchies collapse. But still, it can be adapted to such cases with certain limitations [QP07].

Given the differences between $\mathcal{S R O I} \mathcal{Q}(\mathcal{D})$ axioms and their OWL 2 DL equivalents, in the implementation considered for the experiments, each axiom type is converted in a straightforward 
way to allow comparison. The remaining of this subsection details the conversion of OWL 2 DL axioms for each category as mentioned before. For clarity, Table 7.1 exhibits the category of each axiom type.

\begin{tabular}{ll}
\hline \multicolumn{1}{c}{ Axiom type } & Category \\
\hline Class Assertion & ABox \\
Same Individual & ABox \\
Different Individuals & ABox \\
Object Property Assertion & ABox \\
Negative Object Property Assertion & ABox \\
Data Property Assertion & ABox \\
Negative Data Property Assertion & ABox \\
Has Key & ABox \\
Subclass of & CBox \\
Equivalent Classes & CBox \\
Disjoint Classes & CBox \\
Disjoint Union & CBox \\
Object Property Domain & CBox \\
Data Property Domain & CBox \\
Object Property Range & CBox \\
Data Property Range & CBox \\
Functional Object Property & CBox \\
Inverse Functional Object Property & CBox \\
Reflexive Object Property & CBox \\
Irreflexive Object Property & CBox \\
Functional Data Property & CBox \\
Sub Object Property of & RBox \\
Equivalent Object Properties & RBox \\
Sub Object Property Chain of & RBox \\
Transitive Object Property & RBox \\
Symmetric Object Property & RBox \\
Asymmetric Object Property & RBox \\
Inverse Object Properties & RBox \\
Disjoint Object Properties & RBox \\
Datatype Definition & DBox \\
Sub Data Property of & PBox \\
Equivalent Data Properties & PBox \\
Disjoint Data Properties & PBox \\
\hline
\end{tabular}

Table 7.1: Mapping of OWL 2 DL logical axioms to axiom specificity categories

\section{ABox Category}

As mentioned before, ABox axioms are considered the most specific type of axiom. This group also includes Has Key axioms because they are specific enough to identify an entity uniquely. Hence axioms of this category are the minimal elements of the partial order $\prec_{\text {AxS }}$. 


\section{CBox Category}

These axioms express relations between concepts (classes in OWL 2). For each of these axioms, the conversion procedure extracts a concept inclusions representing that axiom. These concept inclusions are compared via specificity of their left-hand sides, as defined by Qi and Pan [QP07].

Next, this subsection details how this extraction is performed for each type of OWL 2 axiom in the CBox category.

Subclass of and Convertibles These are axioms which can be easily mapped to concept inclusions. This comprises axioms of types: Subclass of, Object Property Domain, Data Property Domain, Object Property Range, Data Property Range, Functional Object Property, Inverse Functional Object Property, Reflexive Object Property, Irreflexive Object Property and Functional Data Property. The mapping is detailed in Table 7.2.

\begin{tabular}{lll}
\hline \multicolumn{1}{c}{ Axiom type } & \multicolumn{1}{c}{ OWL 2 functional syntax } & \multicolumn{1}{c}{ Mapping } \\
\hline Subclass of & SubclassOf(C D) & $\mathrm{C} \sqsubseteq \mathrm{D}$ \\
Object Property Domain & ObjectPropertyDomain(r C) & $\exists \mathrm{r} . \top \sqsubseteq \mathrm{C}$ \\
Data Property Domain & DataPropertyDomain(p C) & $\exists \mathrm{p} . \top \sqsubseteq \mathrm{C}$ \\
Object Property Range & ObjectPropertyRange(r C) & $\top \sqsubseteq \forall \mathrm{r} . \mathrm{C}$ \\
Data Property Range & DataPropertyRange(p C) & $\top \sqsubseteq \forall \mathrm{p} . \mathrm{C}$ \\
Functional Object Property & FunctionalObjectProperty(r) & $\top \sqsubseteq \leq_{1} \mathrm{r} . \top$ \\
Inverse Functional Object Property & InverseFunctionalObjectProperty(r) & $\top \sqsubseteq \leq_{1} \mathrm{r}^{-} \cdot \top$ \\
Reflexive Object Property & ReflexiveObjectProperty(r) & $\top \sqsubseteq \exists \mathrm{r}$.Self \\
Irreflexive Object Property & IrreflexiveObjectProperty(r) & $\top \sqsubseteq \neg \exists \mathrm{r}$.Self \\
Functional Data Property & FunctionalDataProperty(p) & $\top \sqsubseteq \leq_{1} \mathrm{p} . \top$ \\
\hline
\end{tabular}

Table 7.2: Conversion of some $O W L 2 D L$ axioms to concept inclusions

After they are converted into concept inclusions, they are compared regarding axiom specificity with the same rules as in [QP07].

Equivalent Classes, Disjoint Classes and Disjoint Union Axioms In these cases, there are multiple possible concept inclusions that could be derived from a single OWL 2 axiom. Moreover, a Disjoint Union Axiom usually has to be broken down in two parts to be described by the usual DL syntax, one about the disjointness and the other about the union.

For a Equivalent Classes axiom $\alpha$, the generation of a equivalent set concept inclusions is very straightforward: $E_{\alpha}=\{\mathrm{A} \sqsubseteq \mathrm{B} \mid \mathrm{A}, \mathrm{B} \in \operatorname{sig}(\alpha)$ and $\mathrm{A} \neq \mathrm{B}\}$. Further, all elements in $E_{\alpha}$ have the same specificity. Hence, a random element of this set is selected to build the partial order $\prec_{A x S}$.

As for Disjoint Classes and Disjoint Union axioms, the conversion process builds the conjunction of all concepts in their signatures and makes it a subclass of $\perp$. That is, if $\alpha$ is a Disjoint Classes Disjoint Union axiom and $\mathrm{X}_{\alpha}=\sqcap_{\mathrm{A} \in \operatorname{sig}(\alpha)}$, the comparison considers $\mathrm{X}_{\alpha} \sqsubseteq \perp$ instead of $\alpha$ itself.

\section{RBox Category}

For these axioms the comparison extracts the left-hand side (LHS) of a role (chain) inclusion and compares specificity using an analogous criterion as the one used for concept inclusions. The remaining of this subsection details how to obtain a suitable LHS for each RBox axiom type (as defined in Table 7.1).

Sub Object Property of Axioms These axioms can be immediately converted to role inclusions in a DL sense. Hence, their LHS is evaluated as they are given. 
Transitive, Symmetric and Asymmetric Object Property Axioms As these axioms only deal with a single property, this property is return instead of a usual LHS.

Sub Property Chain of In this case, the chain of properties that composes the subproperty is returned as the LHS (see complex role inclusions in Table 2.2).

Equivalent, Inverse and Disjoint Object Properties These axioms could have multiple possible axioms and one cannot simply create a compound property represents a conjunction of roles. Therefore, all properties in their signatures are considered together to decided the partial order relations. Essentially, the process extended the original comparison in the following way: let $\alpha_{1}$ and $\alpha_{2}$ be RBox axioms, also let $R(\alpha)$ be the set of relevant object properties of the axiom $\alpha$, then:

- $\alpha_{1} \equiv \equiv_{\mathrm{AxS}} \alpha_{2}$ if for every $\mathrm{r}_{1} \in R\left(\alpha_{1}\right)$, there exists $\mathrm{r}_{2} \in R\left(\alpha_{2}\right)$ such that $\mathrm{r}_{1} \sqsubseteq \mathrm{r}_{2}$ and $\mathrm{r}_{2} \sqsubseteq \mathrm{r}_{1}$

- $\alpha_{1} \prec \mathrm{AxS}_{2} \alpha_{2}$ if for all $\mathrm{r}_{\mathrm{i}} \in R\left(\alpha_{i}\right) \mathrm{r}_{1} \sqsubseteq \mathrm{r}_{2}$.

- $\alpha_{2} \prec$ AxS $\alpha_{1}$ if for all $\mathrm{r}_{\mathrm{i}} \in R\left(\alpha_{i}\right) \mathrm{r}_{2} \sqsubseteq \mathrm{r}_{1}$.

- Otherwise, $\alpha_{1}$ and $\alpha_{2}$ are incomparable regarding $\prec$ AxS .

This definition maintains the properties of a partial order, in particular transitivity.

\section{PBox Category}

This category also concerns axioms which impose relations between roles, but only those that involve data properties. As with object properties in the RBox category, the comparison method for these axioms considers sets of properties instead of a single one to act as an entity to determine specificity. Next, this subsection describes the management of each of the types of axioms in the DBox category.

Sub Data Property of Axioms This axiom type is very straightforward: one can consider the sub data property given.

Equivalent and Disjoint Data Properties Axioms As with the Equivalent, Inverse and Disjoint Object Properties RBox axiom types, the process evaluates all data properties in the signature simultaneously and compares them using an analogous extension of the specificity comparison.

\section{DBox Category}

The only axiom type in this category is Datatype Definition. Unfortunately, there is no clear a way to compare this type of axiom regarding specificity. Hence, every axiom in this category is incomparable.

\subsubsection{Concept Name Specificity}

There is also an alternative ways to encode specificity besides AxS. One can establish a partial order of concepts: $\mathrm{C}$ is more specific than $\mathrm{D}(\mathrm{C} \prec \sqsubseteq \mathrm{D})$ in $\mathcal{O}$ if $\mathcal{O} \vDash \mathrm{C} \sqsubseteq \mathrm{D}$, but $\mathcal{O} \not \forall \mathrm{D} \sqsubseteq \mathrm{C}$. Let $\operatorname{rank}(\mathrm{C})$ be the rank of the concept name $\mathrm{C}$ for all concept names in $\mathcal{O}$ in the stratification obtained from $\prec_{\sqsubseteq}$, then $\alpha_{1}$ is more concept-specific than $\alpha_{2}$ (written $\alpha_{1} \prec_{\mathrm{CNS}} \alpha_{2}$ ) in $\mathcal{O}$ if $\min _{\mathrm{C} \in \operatorname{sig} \alpha_{1}} \operatorname{rank}(\mathrm{C})<$ $\min _{\mathrm{C} \in \operatorname{sig} \alpha_{2}} \operatorname{rank}(\mathrm{C})$. The concept name specificity (CNS) is the stratification of axioms induced by $\prec_{\mathrm{CNS}}$.

Even though this approach does not display the same syntax sensitivity as axiom specificity for absorption, it fails to capture differences between any pair of axioms whose most specific concepts are at the same level of specificity. Moreover, it also has the same issues when applied to inconsistent 
ontologies as AxS. This dependency on the hierarchy of concept names also implies that the axioms that do not include concepts (such as role inclusions) cannot be ranked by this method.

One notable way in which it differs from AxS is the handling of the assertions: instead of considering all of them equally important, it also considers concepts involved. For instance, if $\mathcal{O}=\{\mathrm{A} \sqsubseteq \mathrm{B}, \mathrm{A}(a), \mathrm{B}(b)\}$, the axioms $\mathrm{A}(a)$ and $\mathrm{B}(b)$ would attain the same rank by axiom specificity, however CNS would indicate that $\mathrm{A}(a)$ is more specific than $\mathrm{B}(b)$.

\subsection{3 $x$-AD Height}

The last class of stratification methods considered is based on the atomic decomposition (discussed in Subsection 2.4.4). These methods use the partial order determined by the dependency relation in the $x$-AD of $\mathcal{O}: \alpha_{1}$ is $x$-preferred to $\alpha_{2}$ (written $\alpha_{1} \prec x$-AD $\alpha_{2}$ ) in $\mathcal{O}$ if $\mathfrak{a}_{1} \prec \mathfrak{a}_{2}$ in $x$ - $\mathrm{AD}(\mathcal{O})$, where $\mathfrak{a}_{i}$ is the atom that contains the axiom $\alpha_{i}$ (since each axiom belongs to exactly one atom, this relation is well-defined). The $x$-AD height is the stratification of axioms induced by $\prec_{x-\mathrm{AD}}$. To shorten the notation, $\perp$-AD height will be referred as BAH and T-AD height as TAH.

Guimaraes and Wassermann defined the AD height only for $T \perp^{*}$-LBMs [GW17]. Their motivation was to use the $\mathrm{AD}$ to retrieve relevant formulas for an input in Belief Change operations, following the studies in Structured Belief Bases [Was01] and Local Change [HW02]. In contrast, this thesis focuses to $T$ and $\perp$-LBMs because the objective is to obtain good dependency relations rather than small modules. Compared to $T \perp^{*}$-locality, $T$ and $\perp$-locality preserve more information about class hierarchies which seem to be beneficial for specificity/generality and importance analysis [Del13]. This effect is lost in with $T \perp^{*}$ LBMs: the $T \perp^{*}-\mathrm{AD}$ of a pure taxonomy has only unconnected atoms.

In particular, $\perp$-LBMs preserve all superclass relationships for the concepts in its signature. In this way, axioms from lower atoms in the $\perp$-AD tend to deal with more general concepts, while higher deal with more specific ones, and the opposite happens with T-AD [Del13]. Hence, one can expect that BAH displays a strong negative correlation with specificity-based metrics, while TAH displays a positive one. The experiments also investigate $\perp-A D$ depth (BAD) and $T-A D$ depth (TAD) to get a clearer picture. The depth metrics are defined analogously to AD heights, but replacing $\prec_{x \text {-AD }}$ with $\succ_{x \text {-AD }}$ in the definition of rank.

The height is a way to estimate which axioms participate of more or fewer modules. An axiom with low height is more likely to be part of more genuine modules and thus also of more general modules, via the dependency relations [GW17]. Proposition 7.2.1 considers the case of removal of an axiom and the question whether modules can be preserved and shows that this metric acts as proxy for importance, if one intends to preserve as many modules as possible.

Proposition 7.2.1. Let $\mathcal{O}$ be an ontology, $x \in\{\perp, \top\}$ a locality notion, $\alpha \in \mathcal{O}$ an axiom and $\Sigma \subseteq \operatorname{sig}(\mathcal{O})$ a signature such that $\alpha \notin x-\operatorname{LBM}(\mathcal{O}, \Sigma)$. Then, $x-\operatorname{LBM}(\mathcal{O} \backslash\{\alpha\}, \Sigma)=x-\operatorname{LBM}(\mathcal{O}, \Sigma)$.

This proposition is an immediate consequence of the fact that $x$-locality w.r.t. $\Sigma$ (and any of its extensions) during the computation of $x$-LBMs, is decided on a "per axiom" basis (see Algorithm 12). As a consequence of Proposition 7.2.1, if $\alpha \prec_{x \text {-AD }} \beta$ then the removal of $\alpha$ affects more $x$-LBMs than the removal of $\beta$. Therefore, axioms with lower heights can be said to be more influential, thus more important.

As the locality-based modules and atomic decomposition are syntax-dependent, so are the $x$-AD heights and depths of the axioms. Therefore, all approaches we consider are syntax-dependent: AxS by ignoring the right-hand sides, CNS by considering only at the most specific concept in the class signature and the ones based on the atomic decomposition due to the definition of syntactic locality.

Concluding this section, the selected stratification methods are compared in Example 7.2.2, which uses the illustrative ontology Koalas (a modified version of the ontology Koala [Del13]) from Table 7.3 and that is inspired by the ontology repair scenario.

Example 7.2.2. Consider the ontology KoalaS shown in Table 7.3 and suppose that one would like to restore coherence (the concept KoalaWithPhD is unsatisfiable) by removing a minimal set of axioms. In other words, the operation $\operatorname{rev}($ KoalaS, $\{$ KoalaWithPhD $\sqsupseteq \perp\}, \emptyset)$ must be performed. 


\begin{tabular}{lrrrrrr}
\hline \multicolumn{1}{c}{ Axiom } & AxS & CNS & BAH & BAD & TAH & TAD \\
\hline$\alpha_{1}:$ Animal $\sqsubseteq \exists$ hasGender.Gender & 4 & 1 & 1 & 3 & 2 & 0 \\
$\alpha_{2}:$ Person $\sqsubseteq$ Animal & 3 & 1 & 2 & 2 & 1 & 1 \\
$\alpha_{3}:$ Person $\sqcap$ Marsupial $\sqsubseteq \perp$ & 1 & 1 & 3 & 1 & 0 & 2 \\
$\alpha_{4}:$ Marsupial $\sqsubseteq$ Animal & 3 & 2 & 2 & 2 & 1 & 1 \\
$\alpha_{5}:$ Koala $\sqsubseteq$ Marsupial & 2 & 1 & 3 & 1 & 0 & 2 \\
$\alpha_{6}:$ KoalaWithPhD $\equiv$ Koala $\sqcap \exists$ hasDegree. $\{P h D\}$ & 1 & 0 & 4 & 0 & 0 & 2 \\
$\alpha_{7}:$ : hasDegree.T $\sqsubseteq$ Person & 2 & 1 & 3 & 1 & 0 & 2 \\
$\alpha_{8}:$ Degree $(M S)$ & 0 & 1 & 0 & 4 & 1 & 0 \\
$\alpha_{9}:$ Degree $(P h D)$ & 0 & 1 & 0 & 4 & 1 & 0 \\
\hline
\end{tabular}

Table 7.3: The KoalaS ontology and the rankings of its axioms according to the five methods considered

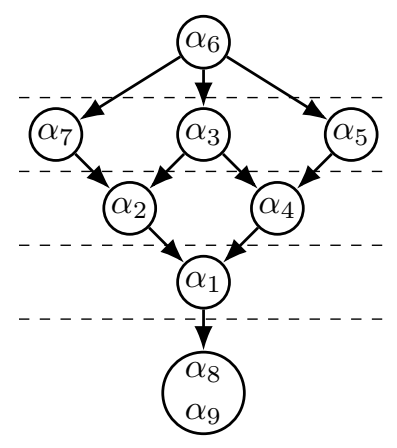

(a) $\perp-A D$ of KoalaS

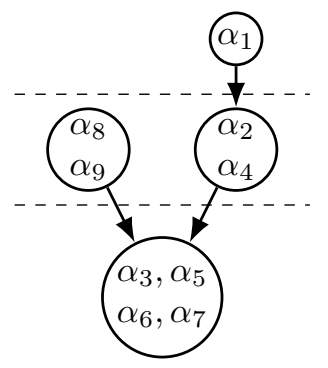

(b) $\top$-AD of KoalaS

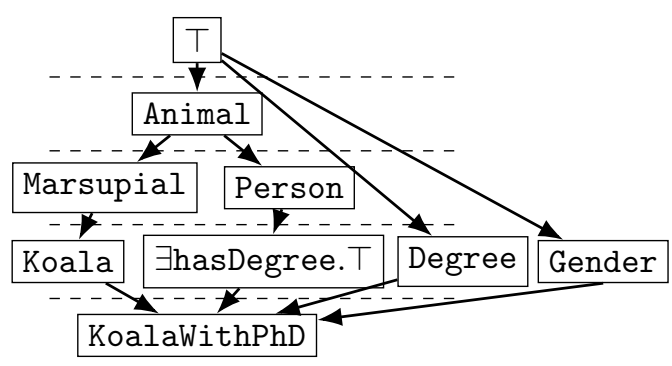

(c) Inferred concept hierarchy

Figure 7.1: Atomic decompositions and inferred concept hierarchy of the KoalaS ontology (dashed lines illustrate possible stratifications)

The candidates for a minimal removal are: $\alpha_{3}, \alpha_{5}, \alpha_{6}$ and $\alpha_{7}$. Removing any of them is enough to make the ontology coherent. TAH and TAH are unable to distinguish between them. Instead, if one follows axiom specificity, either $\alpha_{5}$ or $\alpha_{7}$ would be removed; using CNS or BAD any axiom could be removed, except $\alpha_{6}$, as KoalaWithPhD becomes the most specific concept due to its unsatisfiability. In contrast, since BAH is more likely to favour more general axioms (see Figure 7.1), it would tell us to remove (or fix) $\alpha_{6}$.

According to Table 7.3 and Figure 7.1 BAH and AxS are mostly inverses of each other as expected, that is, ignoring the concept assertions $\alpha_{8}$ and $\alpha_{9}$. Also, BAD is very similar to AxS and CNS. Section 7.5, verifies whether these correlations hold in an ontology corpus using statistical tools.

\subsection{Computing Stratified Constructions}

Benferhat et al. [BBPW17] propose an algorithm to repair ABoxes in stratified ontologies in DL-Lite. This algorithm is also based on Reiter's Hitting Set Tree (as Algorithm 1). Moreover, the authors mention it can be easily extended to other DLs. The method developed establishes a lexicographic (or multiset) ordering of the sets of axioms as defined in Definition 7.3.1.

Definition 7.3.1. Let $\mathcal{O}$ be a stratified ontology and $A, B \subseteq \mathcal{O}$. Then $A \preceq_{\text {lex }} B$, that is $A$ is preferred to $B$ if, for all $0 \leq i<j\left|\mathcal{O}_{i} \cap A\right|=\left|\mathcal{O}_{i} \cap B\right|$ and $\left|\mathcal{O}_{j} \cap A\right| \leq\left|\mathcal{O}_{j} \cap B\right|$. Further if $A \preceq_{\text {lex }} B$ and $B \preceq_{\text {lex }} A$ then $A=_{l e x} B$.

Generalising the algorithm from Benferhat et al. [BBPW17] to arbitrary ontologies and without making distinction between TBox and ABox axioms results in Algorithm 14. 


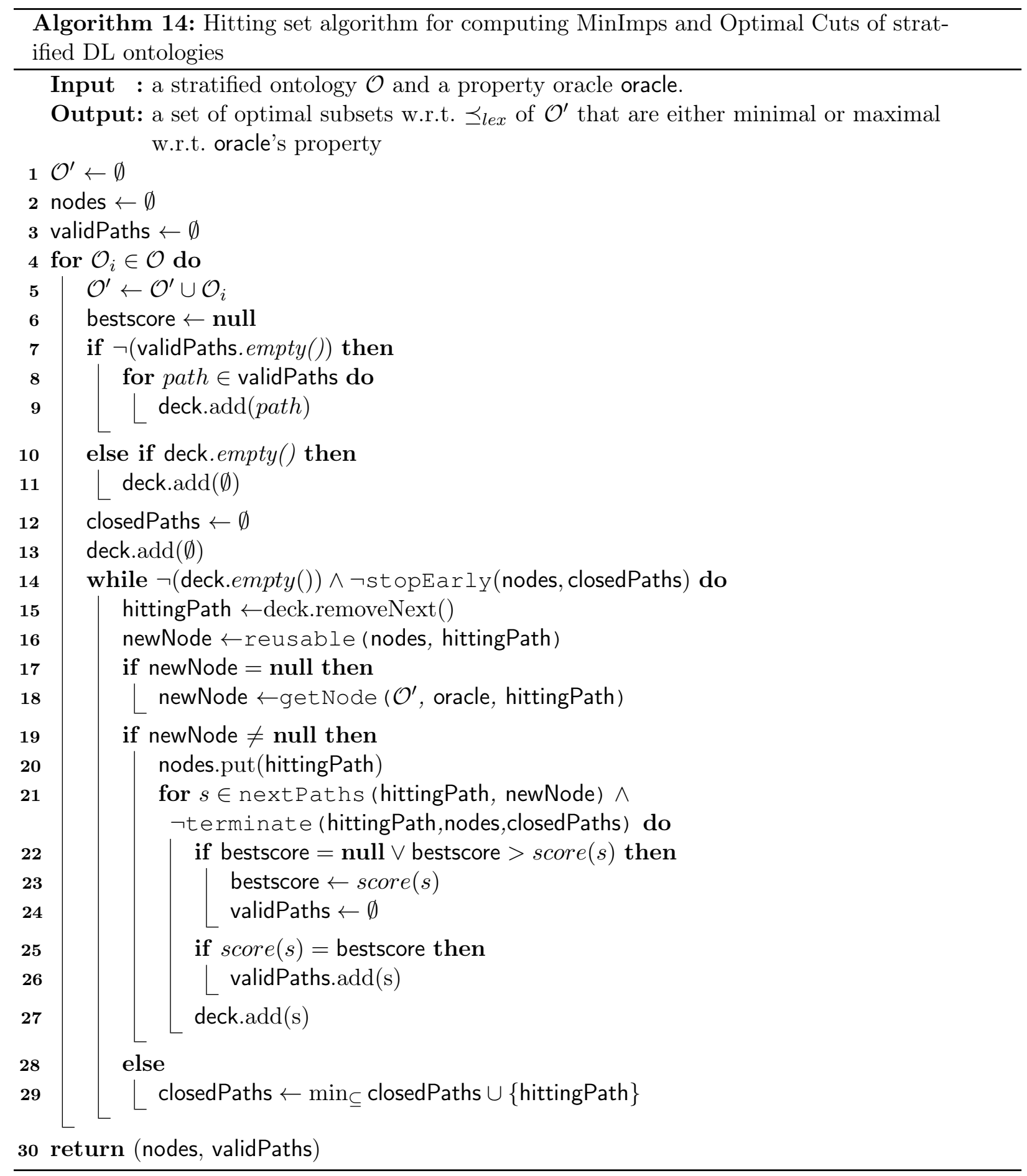


The functions getNode, nextPaths and reusable are implemented according to Algorithm 2.

The stratified version for MaxNons differs considerably from its MinImps counterpart because ranking is over the nodes and not the paths. The intuition for MaxNons is that at each stratum the best nodes are those with maximum cardinality, and they must always be supersets of the nodes computed for the previous strata. Hence, whenever the algorithm passes from one stratum to another, the best nodes are kept and converted into paths, thus forcing the new ones to be supersets of them. Algorithm 15 describes the resulting method. Note that the paths return are always because they do not provide any useful information. The returned in only present for symmetry.

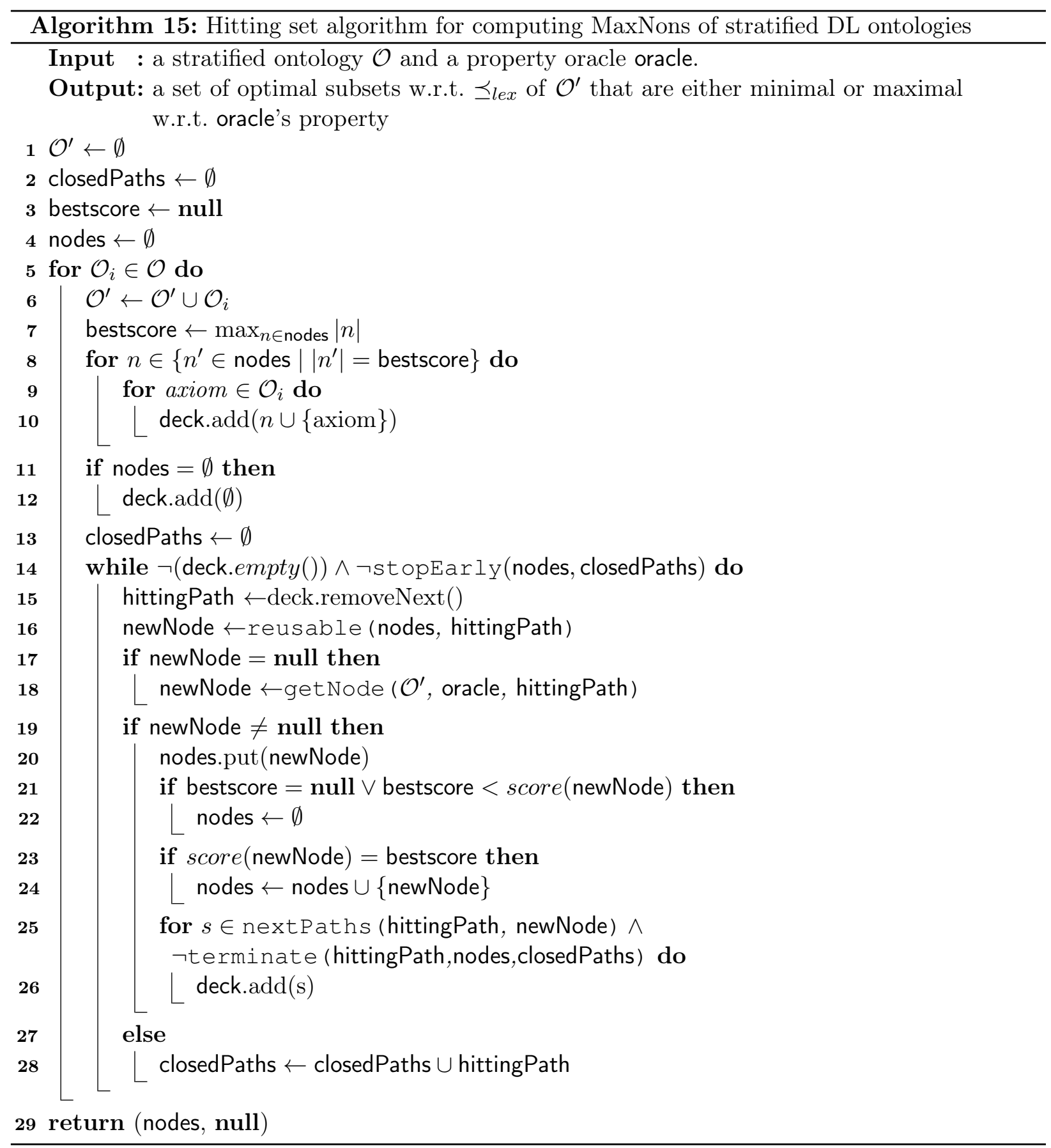

Algorithm 16 is a modularised version of Algorithm 14 inspired in the MU-MI method from Algorithm 13. However, instead of updating the module at each iteration, it only updates the module when a new strata is added.

Algorithm 16 relies on monotonicity on ontology enlargements (Proposition 2.4.8(2)) and on 


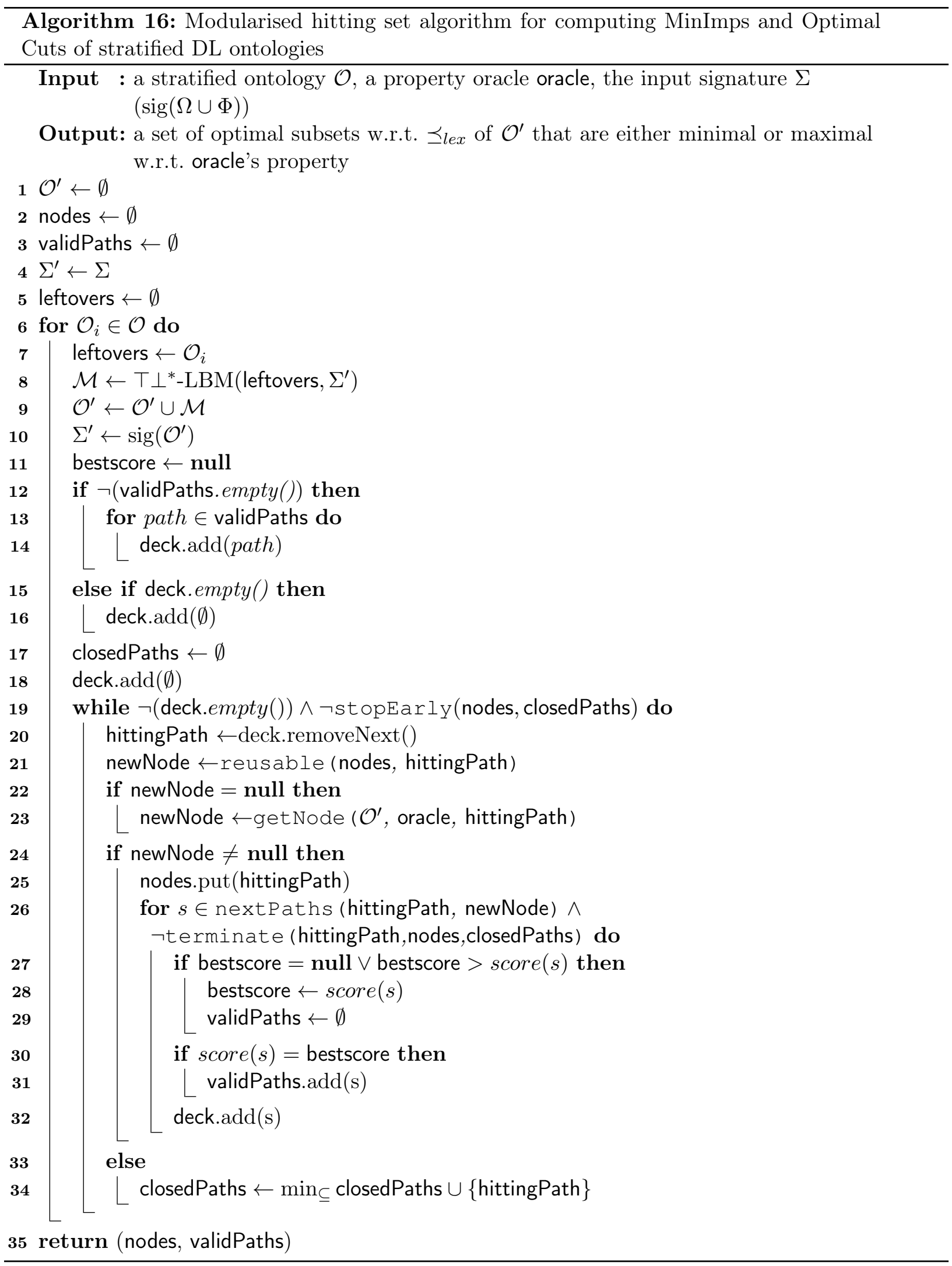


Corollary 4.2.3 to ensure that it can update the module at each strata. At the $k$-th execution of Line 7, let $\mathcal{M}_{k-1}$ be the module from the previous iteration then $\mathcal{M}_{k-1}=\top \perp^{*}-\operatorname{LBM}\left(\mathcal{O}_{0 \leq i \leq k-1}, \Sigma\right)$. Thus, this line computes the module for this iteration: $\mathcal{M}_{k}=\mathcal{M}_{k-1} \cup \top \perp^{*}-\operatorname{LBM}\left(\mathcal{O}_{0 \leq i \leq k} \backslash \mathcal{M}_{k-1}, \Sigma \cup\right.$ $\left.\operatorname{sig}\left(\mathcal{M}_{k-1}\right)\right)$.

In this case, the modularised algorithm for MaxNons (Algorithm 17) also enjoys the same benefits because the ontology considered grows at each step.

It also important to note that, given the correspondence of the closed paths returned in MinImps computation and the nodes computed by MaxNons (they represent $\subseteq$-optimal solutions), the paths returned by Algorithms 14 and 16 correspond to the nodes returned by Algorithms 15 and 17. Note that Algorithm 17 also returns instead of actual paths for the same reason as Algorithm 17: they do not provide anything useful outside the algorithm.

\subsection{Experiments}

There are two separate experiments to address question Q3. The first was already performed by Guimaraes, Sattler and Wassermann [GSW18]. This section presents the results of that experiment using the same data but adding TAD, which was not included in that study. Moreover, the correlation metric used is Kendall's $\tau$ instead of Spearman's $\rho$ because the former is more robust [CD10].

The corpus is the same snapshot of the Bioportal detailed in Subsection 5.4.1. In this case, the experiment evaluated 272 ontologies. These correspond to those that are in the OWL 2 DL profile with at least ten axioms, and whose stratification by all methods took less than 1 hour in the environment is detailed in Table 7.4. Moreover, all of them must have been successfully classified by Hermit (version 1.4.1.513, OWL API 5.1.4). The source code is available as a repository at GitLab ${ }^{1}$.

The second experiment carried on the environment M1 (see Table 5.1) compares AxS and BAH. According to the results of the experiments regarding axiom dispersion (which will be discussed in Subsection 7.5.1), AxS is the best approach among those that represent specificity (although by a thin margin), while BAH is the best AD-based metric. The evaluation computes the best MaxNons for inputs of case T1, using each of these stratification methods, then it compares how many MaxNons each one returned. In this, smaller is better because there would be fewer tied options for a user to select.

Note that the restriction to only cases of type T1 is only due to time constraints. AxS could also be employed in the computation of cases of type T2 (inconsistent related) because the input ontologies themselves are not inconsistent, they only become so if expanded by the input given. Additionally, the experiment could be made with MinImps too, but counting the number of paths returned instead of the nodes. The code and data for this second experiment are via the support material page (https://www.ime.usp.br/ ricardof/thesis_material).

The next section summarises and discusses the results obtained with the experiments as a whole. All in all, it presents results for axiom dispersion (how well the stratification methods distribute axioms in strata), correlation (how the methods are associated statistically) and the results for the novel experiment regarding the filtering of outcomes.

\subsection{Results}

\subsubsection{Axiom Dispersion}

Regarding dispersion, for each method, one can also measure the number of strata in the whole corpus, their relative size and how many pairs of equivalent axioms exist after stratification. The number of strata and their distribution by relative size (the percentage of axioms from the original ontology inside a stratum) show how often small and large strata occur for each method. The indistinguishable axioms rate (IAR) of a stratified ontology is the proportion of pairs of axioms in

\footnotetext{
${ }^{1}$ https://gitlab.com/rfguimaraes/owl2dlstratification/tags/womocoe2018
} 


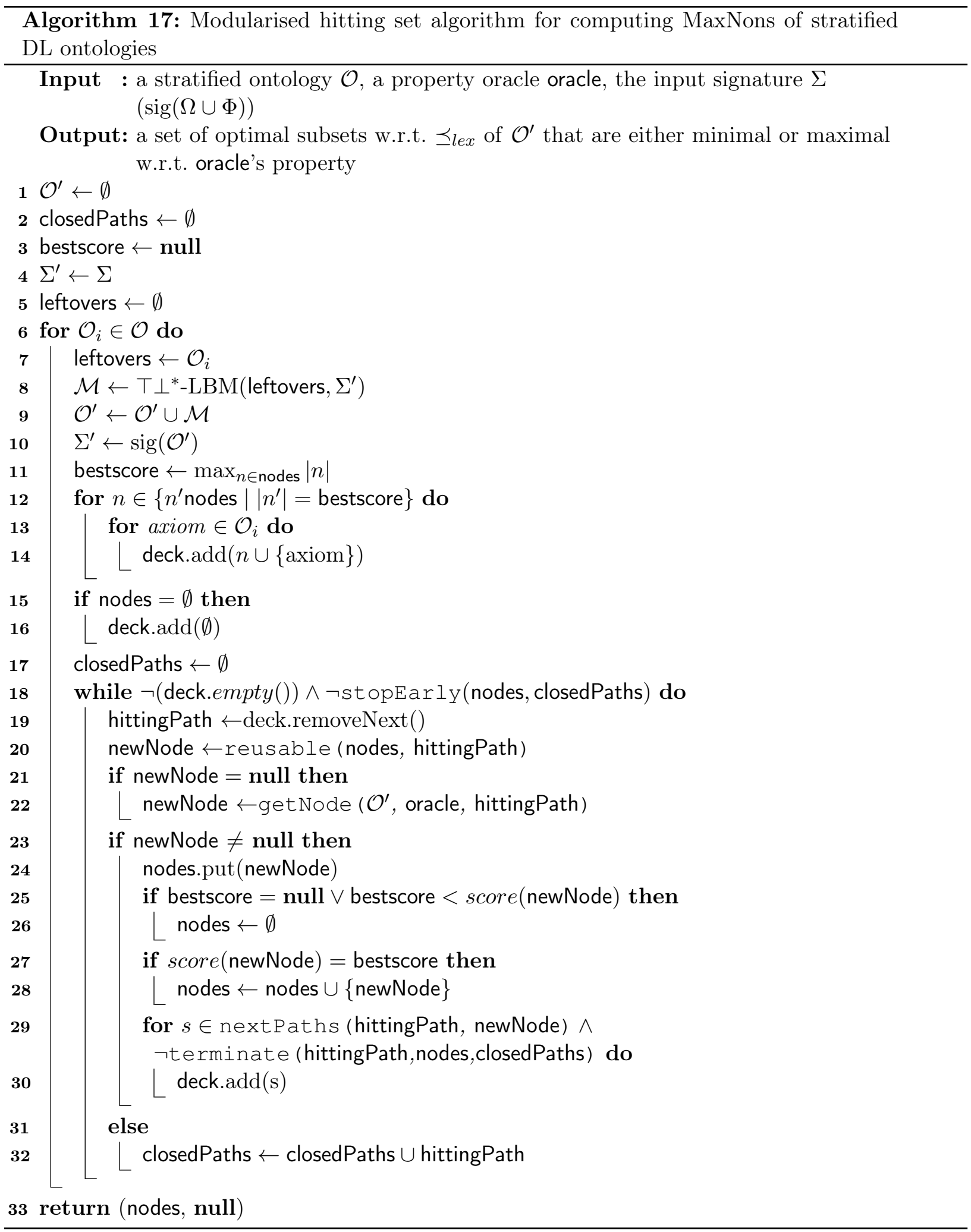




\begin{tabular}{lll}
\hline & Component & Machine 3 \\
\hline \multirow{3}{*}{ CPU } & Model & Intel i5-6200U \\
& Physical cores & 2 \\
& Baseline frequency & $2.3 \mathrm{GHz}$ \\
& Type & DDR 4 \\
Memory & Capacity & $8 \mathrm{~GB}$ \\
& Frequency & $2133 \mathrm{MT} / \mathrm{s}$ \\
\multirow{3}{*}{ Storage } & Access type & $\mathrm{SMP}$ \\
& Type & $\mathrm{HD}$ \\
\multirow{3}{*}{ Operating system } & Capacity & $1 \mathrm{~TB}$ \\
& Kernel & 4.17 \\
Javtribution & Fedora 26 \\
& JRE version & 1.8 \\
& Initial memory pool (-Xms) & $2 \mathrm{~GB}$ \\
& Maximum memory pool (-Xmx) & $6 \mathrm{~GB}$ \\
\hline
\end{tabular}

Table 7.4: Specification of machine 3 (M3) employed in the stratification comparison experiments

the ontology that have axioms with the same rank, i.e., it measures how far the ranking is from a strict total order (the 0 IAR situation).

In the case of the ontology KoalaS from Table 7.3, we have for $\perp$-AD height: 5 strata, with the smaller strata with approximately $11.11 \%$ of the axioms and the largest with around $33.33 \%$ of the axioms, and an IAR of roughly 0.1389 .

This subsection begins with an analysis of dispersion by looking at the cumulative histogram in Figure 7.2. For each method, there is a line that indicates the proportion of strata the method produced that have size up to a determined fraction of the original ontology. Each bin corresponds to increments of $10 \%$ of relative size. Besides, each method produces a different number of strata. This value appears as the " $N$ " near each method label in the legend shows its total number of strata in the corpus.

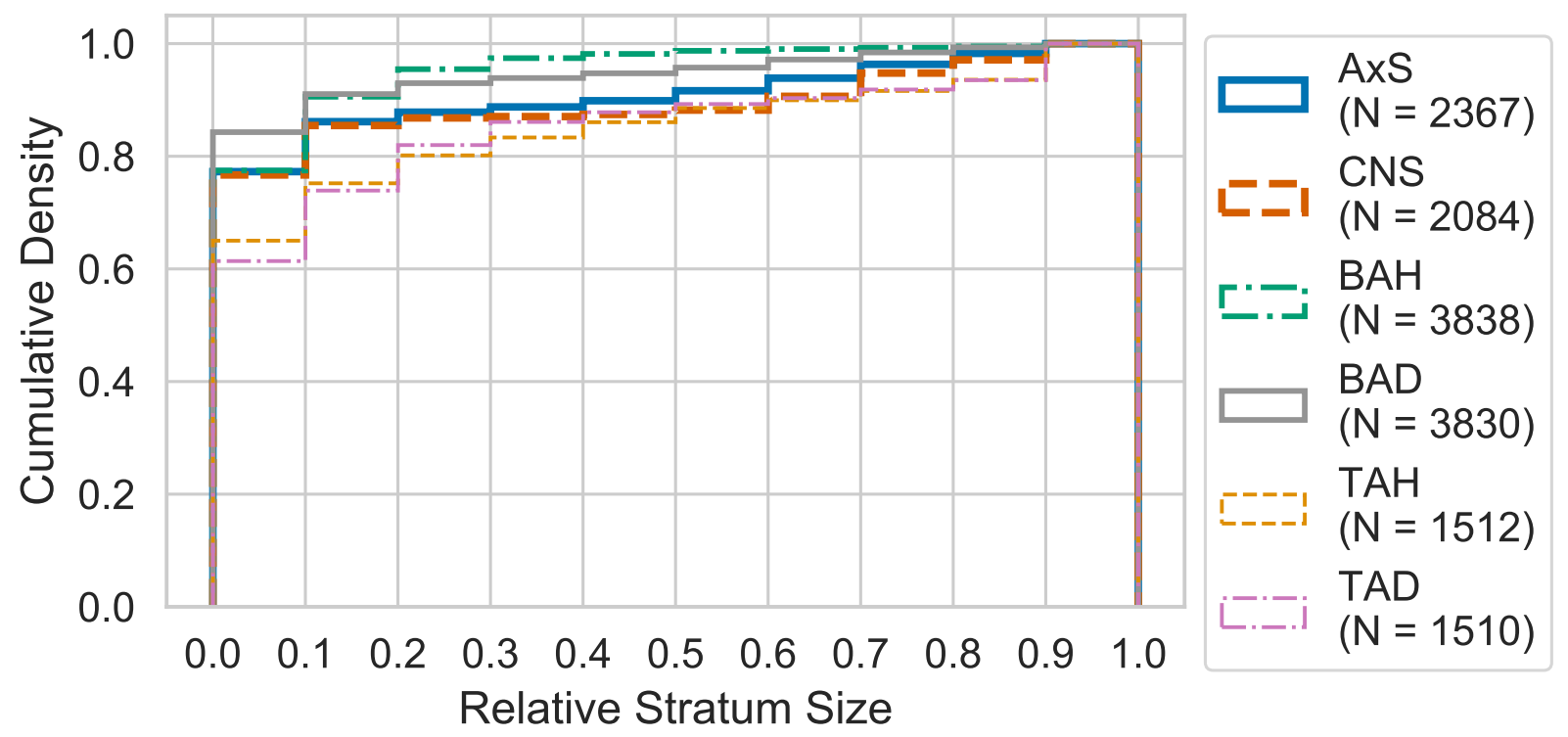

Figure 7.2: Cumulative density histograms of stratum relative sizes over the corpus.

For all methods, at least $60 \%$ of strata produced has size at most $10 \%$ of their original ontologies. 
This result is a good indicator that they produce useful rankings. Notice that BAH and BAD are clear winners regarding the total number of strata generated, while TAH and TAD are notably the worst methods in this aspect. Furthermore, BAH and BAD have the highest proportions of strata below the $20 \%$ mark and are the ones who gain the least as the relative size increases: the proportion of their strata with size $80 \%$ or more of the original ontology is negligible. In contrast, TAH and TAD have the highest proportions of bigger strata; almost $10 \%$ of the strata produced by these methods have a relative size of $90 \%$ or more.

Note that the small differences between the height / depth pairs occur due to atomic decompositions where the distance between the nodes at the top and the bottom is not unique (it depends on the pair of nodes), an example in this case is the T-AD of KoalaS in Figure 7.1b.

The specificity-based approaches not only generated a similar number of strata but also resemble each other in the distribution of strata by relative size, as one can observe by the closeness between their histograms' curves and their trends. Further, their relative stratum size distribution curves are mostly between the ones for $\perp$-AD based methods (which are above) and for T-AD based methods (which are below).

As discussed before, the IAR is one of the most distinctive characteristics to evaluate dispersion, as it gives us a clear indication of how likely it is that each method improves our ability to choose between two axioms. Figure 7.3 displays one violin plot for each method, their external shape (the "violin" is the estimated probability density function using kernel density estimation with smoothing factor given by Scott's rule [Sco92]), cut at the range of the observations. The width of the violin indicates the probability of an ontology having the given IAR value, considering the sample distribution. The internal elements are boxplots marking from the centre to the extremities median, interquartile range and $95 \%$ confidence interval.

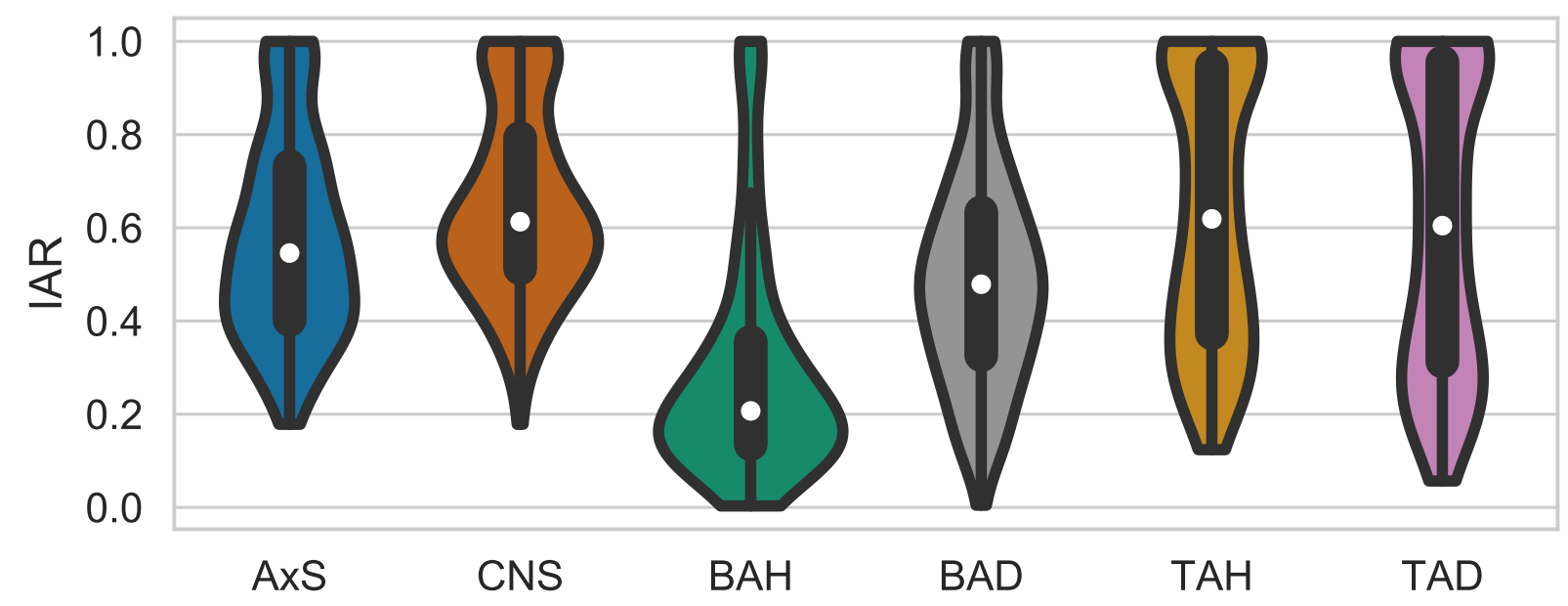

Figure 7.3: Distribution of the indistinguishable axioms rates

From Figure 7.3 , once can infer that $\perp$-AD height is the best approach regarding IAR, with more than a quarter of the 272 ontologies having IAR of less than 0.2. Additionally, the IAR of $\mathrm{BAH}$ in at least $75 \%$ of the corpus is below 0.5 , and the probability density decreases rapidly at higher IAR values. While $\perp-A D$ depth also has 0 IAR in some cases, its distribution is centred on its median, which is close to 0.5 IAR, and it has also attained 1 IAR in the corpus. Excluding BAH, all methods have medians above 0.4 IAR; in other words, for at least half of the ontologies, these methods display IAR of over $40 \%$.

As expected, AxS and CNS have similar IAR distributions. Also, there is a small dominance of AxS over CNS, as the median and quartiles of the former are marked at lower IARs. Still, given the proximity of both distributions and the higher variability of AxS when compared to CNS, there is no proper support to decide which one is better than the other from IAR alone. Neither specificitybased approaches nor T-AD height were able to produce any ranking with IAR smaller than 0.1, 
this is shown by the bottom limits of their (cut) violin plots.

\subsubsection{Correlation}

Here, the correlation between methods is verified in two ways. First, considering each ontology individually and measuring the correlation between the rankings in each. Second, normalising the ranks of the axioms in each ontology to the $[0,1]$ interval, joining the results for the whole corpus and only then measure correlation. The former is called local correlation and the latter corpus correlation.

This subsection begins with the local version. Figure 7.4 shows violin plots (similar to ones presented in Figure 7.3). Each violin plot depicts the distribution of the Kendall's correlation coefficient between two metrics for each ontology in the corpus. Only instances of correlation for which the p-value was below 0.05 are considered. The p-value, in this case, indicates the probability of obtaining the same Kendall's $\tau$ coefficient under the null hypothesis (that is, by chance alone). The value of $\mathrm{N}$ in the legend indicates how many rankings of the 272 passed this significance criterion per pair of methods. As with Figure 7.3, the violins' represent the kernel density estimation function with smoothing via Scott's rule [Sco92].

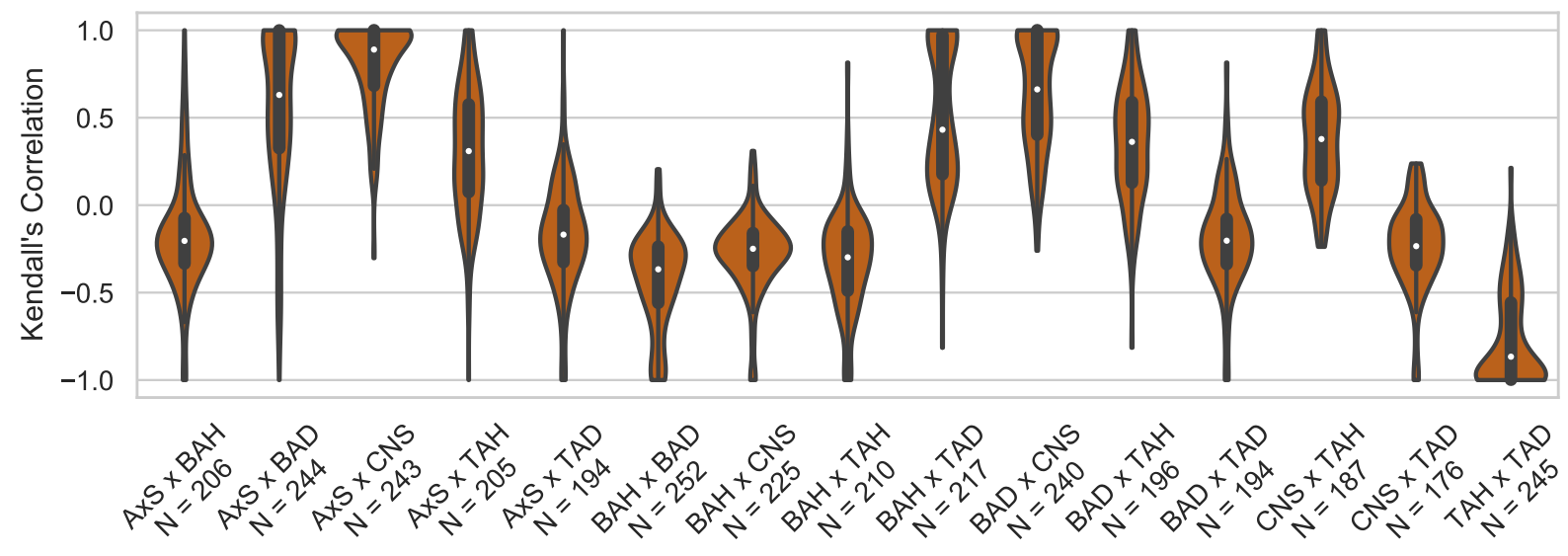

Figure 7.4: Distribution of correlation between the methods (for p-value $<0.05$ ).

As anticipated, CNS often has a very strong positive correlation with AxS and BAD, while AxS and BAD usually display a weaker, but considerable positive correlation. Generally, TAH correlates positively with CNS, AxS and BAD, but weakly. The correlations involving BAH are typically negative and weak (TAD being a moderate positive exception), even though one could expect it to display stronger negative associations with BAD and TAH. In most cases, TAD displays moderate or mild correlations with the other methods, with the opposite signal of TAH, the only exception being the generally inverse strong association with TAH.

In all pairs, most ontologies displayed a significant correlation ( $\mathrm{p}$-value $<0.05$ ), attesting for the reliability of the experiment.

Table 7.5 presents the corpus correlations instead. Note that, as mentioned, the evaluation procedure normalises the ranks of each axiom, in each stratified ontology to the interval $[0,1]$, after that it unifies all rankings for each method, and only them the methods are compared. For brevity, Table 7.5 omits the p-values because they were sufficiently small (the largest at the order of $10^{-} 16$ ). The corpus correlation is a way to measure how robust the correlations seen in Figure 7.4 are in particular, when damping the differences between the ontology sizes.

The corpus correlations Table 7.5 displays some associations akin to those seen in Figure 7.4. For example, the positive correlation between AxS and CNS, note that here it is only moderate. Other notable, but weak relationships are the inverse one between $\mathrm{BAH}$ and $\mathrm{BAD}$, and the direct between BAD and CNS. The others are too weak (below 0.3) to indicate any association reliably. 


\begin{tabular}{llr}
\hline \multicolumn{2}{c}{ Methods } & Kendall's $\tau$ \\
\hline AxS & BAH & -0.1423 \\
AxS & BAD & 0.2683 \\
AxS & CNS & 0.5538 \\
AxS & TAH & -0.0897 \\
AxS & TAD & 0.0878 \\
BAH & BAD & -0.2629 \\
BAH & CNS & -0.1317 \\
BAH & TAH & -0.0091 \\
BAH & TAD & 0.1019 \\
BAD & CNS & 0.3974 \\
BAD & TAH & 0.0536 \\
BAD & TAD & 0.0684 \\
CNS & TAH & 0.0483 \\
CNS & TAD & -0.0192 \\
TAH & TAD & -0.4956 \\
\hline
\end{tabular}

Table 7.5: Corpus correlation values between stratification methods (largest p-value: $2.8208 \times 10^{-16}$ )

\subsubsection{Filtering on Outcomes}

The third evaluation is novel, although more restricted. The last experiment concerning stratification compares AxS and BAH concerning the size of the MaxNons computed using Algorithm 17. Since these algorithms only return the best elements of the MaxNons, the fewer elements they return, the better. This experiment was executed for all inputs of type T1 generated. However, some did not terminate under the timeout of 5 minutes for both methods in M1 (see Table 5.1). The reasoner used was Hermit version 1.4.3.517 with OWL API 5.1.12 and the code is available at https://gitlab.com/rfguimaraes/owl-change/-/tags/0.0.4-alpha2 as a subproject of the experiments for Chapter 6. Excluding these entries, 660 cases remained where the MaxNons has been computed using the modularised Algorithm 1 for MaxNons and Algorithm 17 with AxS and BAH.

Figure 7.5 depicts the relationship between the total number of MaxNons and those considered optimal according to the lexicographic score using AxS. Figure 7.6 is analogous, but with BAH instead. In both figures, the dots are scaled to the number of observations because there are many overlapping pairs.

Figures 7.5 and 7.6 indicate that both stratification methods were effective at reducing the number of candidate MaxNons. While the original method had MaxNons with over 100 elements, AxS never selected more than 12 and BAH returned at most 6 . The data obtained shows that AxS reduced the number of candidates in 580 of the 660 instances, and BAH in 601 of the 660 .

This information however does not allow us to contrapose AxS and BAH. Figures 7.5 and 7.6 look very similar, for instance. Further, both methods had the same number of best candidates in 547 instances; BAH was better than AxS in 68 and worse in the remaining 45. Next, this subsection explores another way to compare AxS and BAH.

Let $i$ be one instance (ontology-input) pair of the 660 used in this experiment. For each instance, there is the number of best MaxNons with AxS, denoted $A x S(i)$, and there is number of best MaxNons with BAH, denoted $B A H(i)$. The difference $B A H(i)-A x S(i)$ in the corpus studied has mean -0.1121, and first, second (median) and third quartiles at 0 . Therefore, one can conclude that these methods were equally good, although BAH has a tiny advantage. 


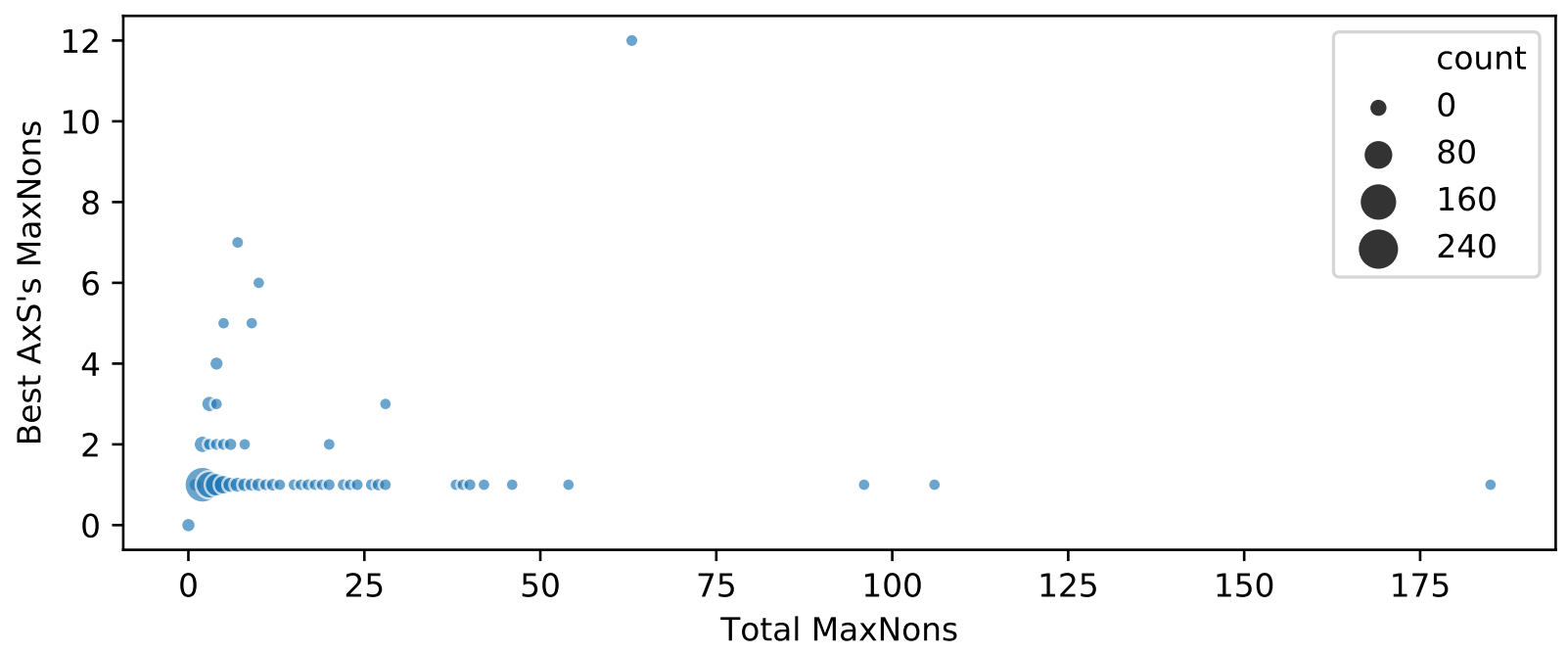

Figure 7.5: Scatter plot relating the number of best MaxNons according to AxS and the original size

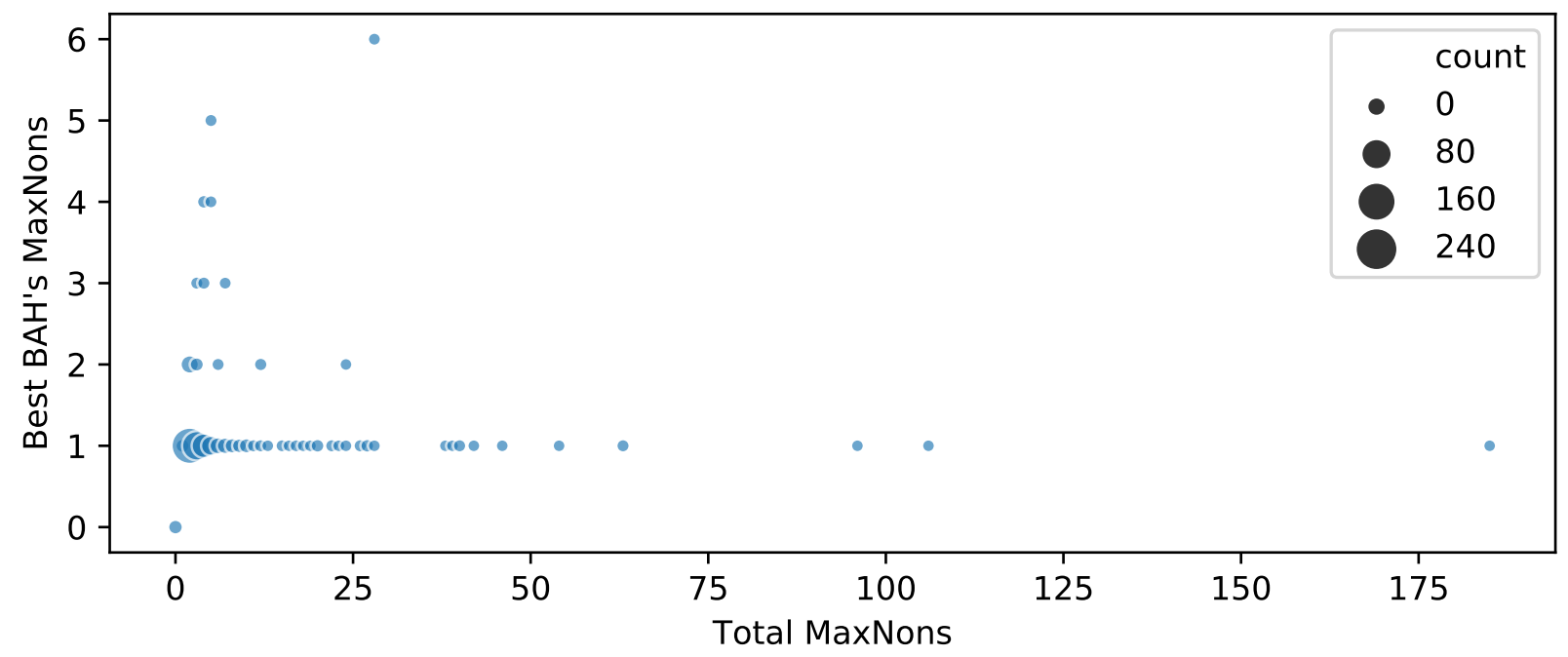

Figure 7.6: Scatter plot relating the number of best MaxNons according to BAH and the original size

\subsubsection{Discussion}

First, a disclaimer: all numerical results in Section 7.5 reflect the behaviour of the methods in a particular corpus. It may be the case that the overall results differ if we consider ontologies from a different domain, or include more ontologies from the same corpus.

The study regarding the effect on the choice problem evaluated modularity-based metrics defined over the atomic decomposition (introduced in Subsection 2.4.4). Using general metrics such as the size of strata and the number of incomparable axioms pairs in a corpus, some modularity approaches looked better than specificity ones and some seemed worse. Additionally, the methods were correlated statistically. While some expectations about the association of methods had been observed (such as positive association between CNS and AxS), many of those were not significant, being moderate or weaker.

For the specific task of computing the best MaxNons, this thesis compared AxS and BAH, still the results were not sufficient to decide which one is better, given that they displayed similar performance. This draw goes in the opposite direction of the other experimental results (especially concerning IAR) in which BAD was clearly better. Although, an implicit benefit of BAH is that it is always tractable, while AxS relies on reasoning. Still, the effects of computational complexity on processing time and memory is left as future work. 
Further, the stratification methods were evaluated using purely numerical metrics. Studies involving real users are needed to indicate whether the choices made via the proposed approaches are helpful in practice. In other words, it might be the case that the quality of the results returned is more important the number of MaxNons returned. 


\section{Chapter 8}

\section{Conclusion}

This thesis investigates the use of modularity strategies to make maintenance of Description Logic more feasible. More specifically, it addresses the high computational costs of ontology repair and the multiplicity of outcomes.

The main contributions consist of a better understanding of the relationship of modules and ontology repair constructions via the Belief Change framework, an evaluation of the impact of modularity in the costs of computing repairs and the study of module-based methods to rank solutions.

As a minor contribution, this work highlights the relationship between Ontology Repair and Belief Change as a continuation of the work by Matos et al. [MGSW19]. Furthermore, to facilitate the collaboration, it develops on the notation for the operations of the later theory that is more accessible for non-experts. The new symbology gets rid of cumbersome symbols for operations and constructions (they are hard to convey and remember) and generalises the set of unwanted sentences $\Omega$, which Ribeiro [Rib13] fixed beforehand while here it is an argument of the belief base operations. Consequently, both expansion and contraction become particular cases of revision, making the presentation of further results much cleaner.

One of the key topics is to determine how modules can connect with Belief Change constructions and postulates. In this sense, this thesis extended the proposal of Suntisrivaraporn et al. [SQJH08] by adapting their algorithm of MinImps computation with LBMs to MaxNons and devising a new method of module-based MinImps which updates the module iteratively. This later algorithm relies on Corollary 4.2.3, which stands as one of the main theoretical results in this work.

One of the key topics is to determine how modules can connect with Belief Change constructions and postulates. In this sense, this thesis extended the proposal of Suntisrivaraporn et al. [SQJH08] by adapting their algorithm of MinImps computation with LBMs to MaxNons and devising a new method of module-based MinImps which updates the module iteratively. This latter method, described in Algorithm 13, relies on Corollary 4.2.3, which stands as one of the main theoretical results in this work.

In addition to these algorithms, this study discusses the impact of modularity on incision and selection functions. In summary, for MinImps, the incision function is the same before and after modularisation, as the domain remains the same. In contrast for MaxNons, the selection function cannot be the same in general, unless the module is the whole ontology. Still, Chapter 4 identifies a bijection between the MaxNons before and after modularisation. Thus, to preserve the result, one needs to obtain a selection function that is isomorphic to the original. Therefore, the main remarks in this aspect is that modularisation via JPMs do not affect the postulates, and, under mild conditions, it does not even change the selection of the outcomes per se.

For the empirical portion of the research, the experiments aim to asses the impact of one of the most prominent modularity approaches, the syntactic locality-based modules, on the computational performance of MinImps and MaxNons algorithms. Note that, these algorithms are reasonerindependent (black-box) and are all based on the hitting set tree algorithm due to Reiter [Rei87]. This approach was selected not only due to the popularity but because previous studies have at- 
tested its efficiency and flexibility.

For the empirical portion of the research, the experiments aim to gauge the impact of one of the most prominent modularity approaches, the syntactic locality-based modules, on the computational performance of MinImps and MaxNons algorithms. Note that, these algorithms are reasonerindependent (black-box) and are all based on the hitting set tree algorithm due to Reiter [Rei87]. This approach was selected not only due to the popularity but because previous studies have attested its efficiency and flexibility [SQJH08, Hor11, CW15].

A key ingredient was missing for the experiments: a corpus. At the time of writing of this thesis, there is not a commonly agreed corpus for testing Belief Change (or Ontology Repair) operations. As discussed in Chapter 5, the approaches in the literature in this matter are ad hoc, with varying designs, generalisation and purpose. These issues motivated another contribution of this work: a corpus generator for Belief Change and Ontology Repair.

Section 5.1 introduced OWL2DL-CCC: a prototype tool that takes as input a set of OWL 2 DL ontologies and a set of error templates in JSON (with a predetermined structure) and outputs a set of ontologies with the faults as specified in the templates, together with supporting files.

The corpus for the experiments was built using the said generator, a dataset of ontologies and a data set of templates (used to modify ontologies and create actual inputs for repair). The ontology dataset consists of snapshot from the BioPortal curated by Matentzoglu and Parsia [MP17]. The case templates were devised based on studies found in the literature of Ontology Repair and related areas [Hor11, BFPS15, CW15].

After elaborating the corpus, the runtime environments were carefully prepared. Hermit and JFact figure as reasoners and the best heuristics for computing a single element of MinImps and MaxNons were determined according to the literature. The quantities measured correspond to the number of calls to the reasoner, processing time and memory consumed.

With the empirical, this thesis attests the benefits of using modules in Belief Base Change (and Ontology Repair) operations, at least in general. The savings on all three quantities evaluated (oracle calls, processing time and maximum memory consumed) were evident, in particular for reasoner calls as this measure is not affected by the overhead of module extraction. Note that in almost all cases, there were inputs for which the modules had a detrimental effect, especially when computing a single solution (the first closed path for MinImps, the first node for MaxNons).

Furthermore, in all cases, modules also reduce the percentage of timeouts. In summary, the verdict is that modules are beneficial for computing complete MinImps and MaxNons, and in more than half of the situations when computing only the "fastest" solution. Further, the correlations results indicate that modules help more in the most resources-consuming instances.

Interestingly, when computing the complete MinImps and MaxNons, MinImps is slightly superior to MaxNons. The opposite occurs when computing the fastest solution but without modularity. For a single solution with modularisation, the difference disappears as it is not possible to tell which is better any longer.

Other measurements were also taken into account to explain when modules are more or less beneficial or when MinImps or MaxNons become significantly better. However, the only clear association was between the gain with the addition of modularity and the absolute value of the quantity measured. That is, more resources consumed (before modularisation) were directly related to better is the impact of using modules. Thus, a proper method to predict when modules are beneficial is still lacking.

Regarding the mitigation of the choice problem, the approach proposed is via stratification methods. Two classification-based strategies were compared with four modularity-based ones. They were evaluated in a general setting, which considers how well it method splits the whole ontology. In this comparison, BAH has performed better than the others, notably when observing its IAR distribution.

More specifically for repair computing, the best classification-based stratification, AxS, was put against with the best of the modularity-based, BAH. Both methods managed to reduce the number of plausible outcomes to a more manageable size. Still, BAH had fewer outcomes in general, likely 
a reflection of its lower IAR distribution, although the benefits over AxS are only marginal.

\subsection{Future Works}

On the theoretical side, future works include investigating which modules are compatible with Pseudo Belief Change, the subarea which investigates operations that not necessarily satisfy inclusion. As with the Gentle Repairs, proposed by Baader et al. [BKNP18], the pseudo-operations allow partial recovery of the information lost when removing a formula [SMRW18, MGSW19].

The main experiment and the focus of Chapter 6, which concerns the impact of modularity, can also be extended in almost all of its parameters. First, it can be reproduced with another ontology dataset to observe how well the results obtained here generalise. Other reasoners, case specifications, modularity approaches are prime candidates for an extension. Another extension is to allow other heuristics in the computation of MinImps and MaxNons to validate the studies from the literature, e.g. due to Horridge [Hor11] and Cóbe and Wassermann [CW15].

A particularly important consideration is that the distribution of module relative sizes was not uniform. Most inputs had modules sizes of $20 \%$ or less of the original ontologies. Moreover, the sizes of $\Omega$ and $\Phi$ could be more vary more by using other templates or extending the case generator to produce such instances.

Also, all experiments in Chapter 6 could be reproduced using $\perp$ and T-LBMs: they are potentially larger but offer less overhead. Also, modularity approaches such as the inseparability-based modules computed via datalog reasoning [RKCH16] (which are smaller than LBMs) can be used for the same purpose as the LBMs. They enjoy most of the theoretical results shown in Chapter 4 and are at most as large as the LBMs. Besides, if one ignores the requirements of preserving Belief Change's postulates such as inclusion, the same studies can also be applied to other modules with yield justification-preserving sets (see Definition 2.4.2), such as the RBMs [NBM13].

Further, a more controlled corpus of ontologies (possibly, generated artificially) can aid in determining when modules are more or less beneficial, and when MinImps fare best against MaxNons methods and vice-versa.

The next step, considering the results obtained, is to find out the characteristics that make modularity more or less effective. In this way, a user or designer one can determine beforehand whether to use the repair techniques as they are or modularise first. The same investigation can be carried out to discover which approach to follow to repair an ontology: MaxNons-based or MinImpsbased. In this direction, it is also interesting to identify the cases in which Algorithm 13, the module update version of MinImps computation, dominates over the SME version.

Regarding the stratification methods, other proposals could be evaluated besides using the class hierarchy and the atomic decomposition. A notable one is the idea of syntactic connectivity or syntactic relevance which figures in Parikh's splitting languages and in Algorithms 7 and 10. Moreover, there is no comparison between stratification methods concerning the resources spent in their computation. Furthermore, the effect of modularity on the stratified algorithms in terms of performance (number of reasoner calls and computational resources) is also left as future work.

In terms of Belief Change, it would be relevant to extend the approaches devised here to other logics, for instance, non-monotonic ones. That would imply, however, in also extending existing modularity approaches to these logics.

Another topic left as future work is in the other direction. More specifically it concerns in the changes over the modular structure of an ontology (for instance via its atomic decomposition) after a repair operation. There are preliminary results in this direction are due to Klinov, Del Vescovo and Schneider [KDS12], and more conclusive answers are needed.

As for the artefacts produced, namely: the corpus generator, the ontology stratification library and the DL base change library, all of them have a wide margin for improvement. For the corpus generator, more concept constructors (see Table 2.1) should be included, only conjunction and disjunction are implemented; as well as optimisations when filtering entities during the instantiation phase. Also, OWL2DL-CCC can only work with inconsistent ontologies limitedly. Further, the 
specification format itself can be extended to provide more flexibility. Finally, better heuristics can be employed to optimise the generation of corpora. The two libraries still need refactoring and better integration with other reasoners outside the OWL API. 


\section{Bibliography}

[AGM85] Carlos E. Alchourrón, Peter Gärdenfors, and David Makinson. On the logic of theory change: Partial meet contraction and revision functions. Journal of Symbolic Logic, 50(2):510-530, 1985. 3, 10, 11, 15, 45, 103

[APW $\left.{ }^{+} 19\right]$ Micah Allen, Davide Poggiali, Kirstie Whitaker, Tom Rhys Marshall, and Rogier A. Kievit. Raincloud plots: a multi-platform tool for robust data visualization. Wellcome Open Research, 4:63, apr 2019. 68

[BBC03] Salem Benferhat, Rania El Baida, and Frédéric Cuppens. A stratification-based approach for handling conflicts in access control. In Elena Ferrari and David F. Ferraiolo, editors, $8^{\text {th }}$ ACM Symposium on Access Control Models and Technologies, SACMAT 2003, Villa Gallia, Como, Italy, June 2-3, 2003, Proceedings, pages 189-195. ACM, 2003. 104

[BBL05] Franz Baader, Sebastian Brandt, and Carsten Lutz. Pushing the EL envelope. In Leslie Pack Kaelbling and Alessandro Saffiotti, editors, IJCAI-05, Proceedings of the Nineteenth International Joint Conference on Artificial Intelligence, Edinburgh, Scotland, UK, July 30 - August 5, 2005, pages 364-369. Professional Book Center, 2005. 2

[BBPW17] Salem Benferhat, Zied Bouraoui, Odile Papini, and Eric Würbel. Prioritized assertional-based removed sets revision of dl-lite belief bases. Ann. Math. Artif. Intell., 79(1-3):45-75, 2017. 3, 104, 109

[BCD $\left.{ }^{+} 93\right]$ Salem Benferhat, Claudette Cayrol, Didier Dubois, Jérôme Lang, and Henri Prade. Inconsistency management and prioritized syntax-based entailment. In Proceedings of the $13^{\text {th }}$ International Joint Conference on Artificial Intelligence (IJCAI 1993), Chambéry, France, August 28 - September 3, 1993, pages 640-647. Morgan Kaufmann, 1993. 103

$\left[\mathrm{BCM}^{+}\right.$03] Franz Baader, Diego Calvanese, Deborah L. Mcguinness, Daniele Nardi, and Peter F. Patel-Schneider. The Description Logic Handbook: Theory, Implementation and Applications. Cambridge University Press, 2003. 37

[BFPS15] Piero A. Bonatti, Marco Faella, Iliana M. Petrova, and Luigi Sauro. A new semantics for overriding in description logics. Artif. Intell., 222:1-48, 2015. 53, 63, 122

[BH95] Franz Baader and Bernhard Hollunder. Priorities on defaults with prerequisites, and their application in treating specificity in terminological default logic. Journal of Automated Reasoning, 15(1):41-68, 1995. 103, 104

[BHLS17] Franz Baader, Ian Horrocks, Carsten Lutz, and Uli Sattler. An Introduction to Description Logic. Cambridge University Press, 2017. 2, 7, 8

[BKNP18] Franz Baader, Francesco Kriegel, Adrian Nuradiansyah, and Rafael Peñaloza. Making repairs in description logics more gentle. In Michael Thielscher, Francesca Toni, 
and Frank Wolter, editors, Principles of Knowledge Representation and Reasoning: Proceedings of the Sixteenth International Conference, KR 2018, Tempe, Arizona, 30 October - 2 November 2018, pages 319-328. AAAI Press, 2018. 13, 123

[BP10] Franz Baader and Rafael Peñaloza. Axiom pinpointing in general tableaux. J. Log. Comput., 20(1):5-34, 2010. 37

[BPS07] Franz Baader, Rafael Peñaloza, and Boontawee Suntisrivaraporn. Pinpointing in the description logic EL + . In Proceedings of the 20 $0^{\text {th }}$ International Workshop on Description Logics (DL 2007), Brixen-Bressanone, near Bozen-Bolzano, Italy, 8-10 June, 2007, volume 250 of CEUR Workshop Proceedings. CEUR-WS.org, 2007. 3, 37, 39

[BS08] Franz Baader and Boontawee Suntisrivaraporn. Debugging SNOMED CT using axiom pinpointing in the description logic EL + . In Ronald Cornet and Kent A. Spackman, editors, Proceedings of the Third International Conference on Knowledge Representation in Medicine, Phoenix, Arizona, USA, May 31st - June 2nd, 2008, volume 410 of CEUR Workshop Proceedings. CEUR-WS.org, 2008. 26, 37, 38, 60

[CD10] Christophe Croux and Catherine Dehon. Influence functions of the spearman and kendall correlation measures. Statistical Methods \& Applications, 19(4):497-515, may 2010. 67,113

[CHKS08] Bernardo Cuenca Grau, Ian Horrocks, Yevgeny Kazakov, and Ulrike Sattler. Modular Reuse of Ontologies: Theory and Practice. Journal of Artificial Intelligence Research, $31: 273-318,2008$. 6, 26, 27, 28, 31

[CHWKS10] Bernardo Cuenca Grau, Christian Halaschek-Wiener, Yevgeny Kazakov, and Boontawee Suntisrivaraporn. Incremental classification of description logics ontologies. Journal of Automated Reasoning, 44(4):337-369, 2010. 26

[CLMW17] Jieying Chen, Michel Ludwig, Yue Ma, and Dirk Walther. Zooming in on ontologies: Minimal modules and best excerpts. In Proceedings of the $16^{\text {th }}$ International Semantic Web Conference (ISWC 2017), Vienna, Austria, October 21-25, 2017, volume 10587 of Lecture Notes in Computer Science, pages 173-189. Springer, 2017. 26, 27

[Cób14] Raphael Cóbe. Integração entre múltiplas ontologias: reúso e gerência de conflitos. PhD thesis, Universidade de São Paulo, 2014. 20, 21, 39, 60, 103, 104

[CP00] Samir Chopra and Rohit Parikh. Relevance sensitive belief structures. Annals of Mathematics and Artificial Intelligence, 28(1-4):259-285, 2000. 25

[CPSK06] Bernardo Cuenca Grau, Bijan Parsia, Evren Sirin, and Aditya Kalyanpur. Modularity and web ontologies. In Proceedings of the $10^{\text {th }}$ International Conference on Principles of Knowledge Representation and Reasoning (KR 2006), Lake District of the United Kingdom, June 2-5, 2006, pages 198-209. AAAI Press, 2006. 26

[CW15] Raphael Cóbe and Renata Wassermann. Ontology repair through partial meet contraction. In Proceedings of the International Workshop on Defeasible and Ampliative Reasoning, DARe 2015, co-located with the 24th International Joint Conference on Artificial Intelligence (IJCAI 2015), Buenos Aires, Argentina, July 27, 2015., volume 1423 of CEUR Workshop Proceedings. CEUR-WS.org, 2015. 19, 20, 21, 23, 33, 37, 53, $61,63,122,123$

[Del13] Chiara Del Vescovo. The Modular Structure of an Ontology: Atomic Decomposition and its Applications. PhD thesis, University of Manchester, 2013. 8, 26, 27, 28, 29, 33, 34, 35, 38, 39, 108 
$\left[\mathrm{DKP}^{+}{ }^{12}\right]$ Chiara Del Vescovo, Pavel Klinov, Bijan Parsia, Ulrike Sattler, Thomas Schneider, and Dmitry Tsarkov. Syntactic vs. semantic locality: How good is a cheap approximation? In Proceedings of the $6^{\text {th }}$ International Workshop on Modular Ontologies (WOMO 2012), Graz, Austria, July 24, 2012, volume 875 of CEUR Workshop Proceedings. CEUR-WS.org, 2012. 28, 38

[DPSS11] Chiara Del Vescovo, Bijan Parsia, Ulrike Sattler, and Thomas Schneider. The modular structure of an ontology: Atomic decomposition. In Proceedings of the 22 ${ }^{\text {nd }}$ International Joint Conference on Artificial Intelligence (IJCAI 2011), Barcelona, Catalonia, Spain, July 16-22, 2011, pages 2232-2237. IJCAI/AAAI, 2011. 6, 26, 33, 34

[DQ15] Jianfeng Du and Guilin Qi. Tractable computation of representative abox repairs in description logic ontologies. In Proceedings of the $8^{\text {th }}$ International Conference on Knowledge Science, Engineering and Management (KSEM 2015), Chongqing, China, October 28-30, 2015, volume 9403 of Lecture Notes in Computer Science, pages 28-39. Springer, 2015. 3

[DQJ09] Jianfeng Du, Guilin Qi, and Qiu Ji. Goal-directed module extraction for explaining OWL DL entailments. In Lecture Notes in Computer Science, pages 163-179. Springer Berlin Heidelberg, 2009. 27

[FFK06] Marcelo A. Falappa, Eduardo L. Fermé, and Gabriele Kern-Isberner. On the logic of theory change: Relations between incision and selection functions. In Gerhard Brewka, Silvia Coradeschi, Anna Perini, and Paolo Traverso, editors, Proceedings of the $1^{r^{\text {th }}}$ European Conference on Artificial Intelligence (ECAI 2006) including Prestigious Applications of Intelligent Systems (PAIS 2006), Riva del Garda, Italy, August 29 September 1, 2006, volume 141 of Frontiers in Artificial Intelligence and Applications, pages 402-406. IOS Press, 2006. 14, 15

[FH11] Eduardo Fermé and Sven Ove Hansson. AGM 25 years. Journal of Philosophical Logic, 40(2):295-331, 2011. 3, 10

[Flo06] Giorgos Flouris. On Belief Change and Ontology Evolution. PhD thesis, University of Crete, 2006. 3, 11

[FUV83] Ronald Fagin, Jeffrey D. Ullman, and Moshe Y. Vardi. On the semantics of updates in databases. In Proceedings of the $2^{\text {nd }} A C M$ Symposium on Principles of Database Systems (SIGACT-SIGMOD 1983), Atlanta, Georgia, USA, March 21-23, 1983, pages 352-365. ACM, 1983. 103

[Gär88] Peter Gärdenfors. Knowledge in Flux: Modelling the Dynamics of Epistemic States. MIT Press, 1988. 103

[Gar89] James W. Garson. Modularity and Relevant Logic. Notre Dame Journal of Formal Logic, 30(2):207-223, 1989. 25, 26

[GKW14] William Gatens, Boris Konev, and Frank Wolter. Lower and upper approximations for depleting modules of description logic ontologies. In Proceedings of the $21^{\text {st }}$ European Conference on Artificial Intelligence (ECAI 2014), Prague, Czech Republic, August 18-22, 2014, volume 263 of Frontiers in Artificial Intelligence and Applications, pages 345-350. IOS Press, 2014. 26

[Gru93] Thomas R. Gruber. A translation approach to portable ontology specifications. Knowledge Acquisition, 5(2):199-220, jun 1993. 1 
[GSW18] Ricardo Guimarães, Uli Sattler, and Renata Wassermann. Ontology stratification methods: A comparative study. In Kristijonas Cyras, Tiago Oliveira, Matthew Williams, Loris Bozzato, Martin Homola, Till Mossakowski, and Antoine Zimmermann, editors, Proceedings of the Joint Proceedings of Reasoning with Ambiguous and Conflicting Evidence and Recommendations in Medicine (MedRACER 2018) and the $3^{\text {rd }}$ International Workshop on Ontology Modularity, Contextuality, and Evolution (WOMoCoE 2018) co-located with the $16^{\text {th }}$ International Conference on Principles of Knowledge Representation and Reasoning (KR 2018), Tempe, Arizona, USA, October 29th, 2018., volume 2237 of CEUR Workshop Proceedings, pages 51-62. CEURWS.org, 2018. 5, 35, 38, 40, 103, 104, 113

[GW17] Ricardo Guimarães and Renata Wassermann. Local change in ontologies with atomic decomposition. In Proceedings of the $3^{\text {rd }}$ Joint Ontology Workshops (JOWO 2017), Bozen-Bolzano, Italy, September 21-23, 2017, volume 2050 of CEUR Workshop Proceedings. CEUR-WS.org, 2017. 26, 34, 39, 103, 108

[Han91] Sven Ove Hansson. Belief contraction without recovery. Studia Logica, 50(2):251-260, 1991. 3,10

[Han93] Sven Ove Hansson. Reversing the Levi identity. Journal of Philosophical Logic, 22(6):637-669, 1993. 11

[Han94a] Sven Ove Hansson. Kernel contraction. Journal of Symbolic Logic, 59(03):845-859, 1994. 11,14

[Han94b] Sven Ove Hansson. Taking Belief Bases Seriously. In Logic and Philosophy of Science in Uppsala: Papers from the $9^{\text {th }}$ International Congress of Logic, Methodology and Philosophy of Science, pages 13-28. Springer Netherlands, Dordrecht, 1994. 3, 10, 11

[Han99] Sven Ove Hansson. A Textbook of Belief Dynamics: Theory Change and Database Updating. Applied Logic Series. Kluwer Academic Publishers, 1999. 10

$\left[\mathrm{HKP}^{+}\right.$09] Pascal Hitzler, Markus Krötzsch, Bijan Parsia, Peter F. Patel-Schneider, and Sebastian Rudolph. OWL 2 Web Ontology Language: Primer. W3C Recommendation, 27 October 2009. Available at http://www.w3.org/TR/owl2-primer/. 7

[Hor11] Matthew Horridge. Justification based explanation in ontologies. PhD thesis, University of Manchester, 2011. 3, 4, 5, 12, 19, 20, 21, 23, 26, 27, 28, 32, 37, 38, 53, 63, 88, 122,123

[HvHtT05] Zhisheng Huang, Frank van Harmelen, and Annette ten Teije. Reasoning with inconsistent ontologies. In Proceedings of the Nineteenth International Joint Conference on Artificial Intelligence (IJCAI 2005), Edinburgh, Scotland, UK, July 30 - August 5, 2005, pages 454-459. Professional Book Center, 2005. 33

[HW02] Sven Ove Hansson and Renata Wassermann. Local change. Studia Logica, 70(1):49-76, 2002. $3,11,14,15,25,26,108$

[IMMP17] Alexey Ignatiev, João Marques-Silva, Carlos Mencía, and Rafael Peñaloza. Debugging EL + ontologies through horn MUS enumeration. In Alessandro Artale, Birte Glimm, and Roman Kontchakov, editors, Proceedings of the 30th International Workshop on Description Logics, Montpellier, France, July 18-21, 2017, volume 1879 of CEUR Workshop Proceedings. CEUR-WS.org, 2017. 37

[JBQ19] Qiu Ji, Khaoula Boutouhami, and Guilin Qi. Resolving logical contradictions in description logic ontologies based on integer linear programming. IEEE Access, 7:7150071510, 2019. 20 
[JQH09] Qiu Ji, Guilin Qi, and Peter Haase. A relevance-directed algorithm for finding justifications of DL entailments. In Proceedings of the $4^{\text {th }}$ Asian Conference (ASWC 2009), Shanghai, China, December 6-9, 2009, volume 5926 of Lecture Notes in Computer Science, pages 306-320. Springer, 2009. 19, 33, 37

[Jun01] Ulrich Junker. QuickXPlain: Conflict Detection for Arbitrary Constraint Propagation Algorithms. In Christian Bessiere, Francois Laburthe, Pedro Meseguer, Jean-Charles Regin, Francesca Rossi, Babara Smith, and Toby Walsh, editors, Workshop on Modelling and Solving Problems with Constraints (CONS-1), co-located with the International Joint Conference on Artificial Intelligence (IJCAI- 2001), Seattle WA, August 2001., 2001. 23

[Kal06] Aditya Kalyanpur. Debugging and repair of OWL ontologies. PhD thesis, University of Maryland, College Park, MD, USA, 2006. 3

[Kaz08] Yevgeny Kazakov. RIQ and SROIQ are harder than SHOIQ. In Gerhard Brewka and Jérôme Lang, editors, Principles of Knowledge Representation and Reasoning: Proceedings of the Eleventh International Conference, KR 2008, Sydney, Australia, September 16-19, 2008, pages 274-284. AAAI Press, 2008. 2

[KDS12] Pavel Klinov, Chiara Del Vescovo, and Thomas Schneider. Incrementally updateable and persistent decomposition of OWL ontologies. In Proceedings of OWL: Experiences and Directions Workshop 2012 (OWLED 2012), Heraklion, Crete, Greece, May 27-28, 2012, volume 849 of CEUR Workshop Proceedings. CEUR-WS.org, 2012. 39, 123

[Ken38] M. G. Kendall. A new measure of rank correlation. Biometrika, 30(1-2):81-93, jun 1938. 67

[KLPW10] Boris Konev, Carsten Lutz, Denis K. Ponomaryov, and Frank Wolter. Decomposing description logic ontologies. In Proceedings of the $12^{\text {th }}$ International Conference on Principles of Knowledge Representation and Reasoning (KR 2010), Toronto, Ontario, Canada, May 9-13, 2010. AAAI Press, 2010. 25, 26

[KLWW09] Boris Konev, Carsten Lutz, Dirk Walther, and Frank Wolter. Formal properties of modularisation. In Modular Ontologies: Concepts, Theories and Techniques for Knowledge Modularization, Lecture Notes in Computer Science, pages 25-66. Springer Berlin Heidelberg, 2009. 25, 26, 29

[KM07] George Kourousias and David Makinson. Parallel interpolation, splitting, and relevance in belief change. Journal of Symbolic Logic, 72(3):994-1002, 2007. 25

[KPS ${ }^{+}$09] Roman Kontchakov, Luca Pulina, Ulrike Sattler, Thomas Schneider, Petra Selmer, Frank Wolter, and Michael Zakharyaschev. Minimal module extraction from dl-lite ontologies using QBF solvers. In Proceedings of the $21^{\text {st }}$ International Joint Conference on Artificial Intelligence (IJCAI 2009), Pasadena, California, USA, July 11-17, 2009, pages $836-841,2009.25,26,29,30$

[KPSG06] Aditya Kalyanpur, Bijan Parsia, Evren Sirin, and Bernardo Cuenca Grau. Repairing unsatisfiable concepts in OWL ontologies. In The Semantic Web: Research and Applications, $3^{\text {rd }}$ European Semantic Web Conference, ESWC 2006, Budva, Montenegro, June 11-14, 2006, Proceedings, volume 4011 of Lecture Notes in Computer Science, pages 170-184. Springer, 2006. 53

[KPSH05] Aditya Kalyanpur, Bijan Parsia, Evren Sirin, and James A. Hendler. Debugging unsatisfiable classes in OWL ontologies. J. Web Semant., 3(4):268-293, 2005. 19 
[KWZ10] Roman Kontchakov, Frank Wolter, and Michael Zakharyaschev. Logic-based ontology comparison and module extraction, with an application to DL-Lite. Artificial Intelligence, 174(15):1093-1141, 2010. 26

[Mak09] David Makinson. Propositional relevance through letter-sharing. Journal of Applied Logic, 7(4):377-387, 2009. 25

[Mat16] Nicolas Matentzoglu. Module-based classification of OWL ontologies. PhD thesis, University of Manchester, UK, 2016. 2, 4, 26, 35, 38, 53, 58, 59, 60, 61, 63, 67

[MBP13a] Nicolas Matentzoglu, Samantha Bail, and Bijan Parsia. A corpus of OWL DL ontologies. In Thomas Eiter, Birte Glimm, Yevgeny Kazakov, and Markus Krötzsch, editors, Informal Proceedings of the 26th International Workshop on Description Logics, Ulm, Germany, July 23 - 26, 2013, volume 1014 of CEUR Workshop Proceedings, pages 829-841. CEUR-WS.org, 2013. 53

[MBP13b] Nicolas Matentzoglu, Samantha Bail, and Bijan Parsia. A snapshot of the OWL web. In The Semantic Web - ISWC 2013 - 12 ${ }^{\text {th }}$ International Semantic Web Conference, Sydney, NSW, Australia, October 21-25, 2013, Proceedings, Part I, volume 8218 of Lecture Notes in Computer Science, pages 331-346. Springer, 2013. 63

[MGSW19] Vinícius Bitencourt Matos, Ricardo Ferreira Guimarães, Yuri David Santos, and Renata Wassermann. Pseudo-contractions as gentle repairs. In Carsten Lutz, Uli Sattler, Cesare Tinelli, Anni-Yasmin Turhan, and Frank Wolter, editors, Description Logic, Theory Combination, and All That - Essays Dedicated to Franz Baader on the Occasion of His 60th Birthday, volume 11560 of Lecture Notes in Computer Science, pages 385-403. Springer, 2019. xi, 6, 11, 12, 13, 14, 15, 27, 121, 123

[MMS14] Kodylan Moodley, Thomas Meyer, and Uli Sattler. Practical defeasible reasoning for description logics. In STAIRS 2014 - Proceedings of the tht European Starting AI Researcher Symposium, Prague, Czech Republic, August 18-22, 2014, volume 264 of Frontiers in Artificial Intelligence and Applications, pages 191-200. IOS Press, 2014. 53

[Moo10] Kodylan Moodley. Debugging and repair of description logic ontologies. Master's thesis, University of KwaZulu-Natal, 2010. 20, 26, 32, 37, 38, 60, 61, 88

[MP17] Nicolas Matentzoglu and Bijan Parsia. Bioportal snapshot 30.03.2017, 2017. 63, 122

[MW14] Francisco Martín-Recuerda and Dirk Walther. Axiom dependency hypergraphs for fast atomic decomposition of ontologies. In Proceedings of the $2^{\text {rth }}$ International Workshop on Description Logics (DL 2014), Vienna, Austria, July 17-20, 2014, volume 1193 of CEUR Workshop Proceedings, pages 299-310. CEUR-WS.org, 2014. 34, 39

[NBM13] Riku Nortje, Katarina Britz, and Thomas Meyer. Reachability modules for the description logic SRIQ. In Logic for Programming, Artificial Intelligence, and Reasoning, pages 636-652. Springer Berlin Heidelberg, 2013. 27, 29, 123

[Neb91] Bernhard Nebel. Belief revision and default reasoning: Syntax-based approaches. In Proceedings of the $2^{\text {nd }}$ International Conference on Principles of Knowledge Representation and Reasoning (KR 1991), Cambridge, MA, USA, April 22-25, 1991, pages 417-428. Morgan Kaufmann, 1991. 103

[Nor11] Riku Nortjé. Module extraction for inexpressive description logics. PhD thesis, University of South Africa, 2011. 37, 53, 60 
[OP18] Ana Ozaki and Rafael Peñaloza. Consequence-based axiom pinpointing. In Lecture Notes in Computer Science, pages 181-195. Springer International Publishing, 2018. 19

[Par99] Rohit Parikh. Beliefs, Belief Revision, and Splitting Languages. In Logic, Language and Computation, volume 2, chapter 13, pages 266-278. Center for the Study of Language and Information, Stanford, CA, USA, 1999. 25, 33

[Par09] Bijan Parsia. Topic-sensitive belief revision. PhD thesis, University of Maryland, 2009. 25

[Peñ09] Rafael Peñaloza. Axiom pinpointing in description logics and beyond. PhD thesis, Dresden University of Technology, 2009. 3, 19, 39

[Pep08] Pavlos Peppas. Belief revision. In Handbook of Knowledge Representation, pages 317-359. Elsevier, 2008. 10

[PMIMS17] Rafael Peñaloza, Carlos Mencía, Alexey Ignatiev, and Joao Marques-Silva. Lean kernels in description logics. In Semantic Web, pages 518-533. Springer International Publishing, 2017. 26, 27

[PS09] Christine Parent and Stefano Spaccapietra. An overview of modularity. In Modular Ontologies: Concepts, Theories and Techniques for Knowledge Modularization, volume 5445 of Lecture Notes in Computer Science, pages 5-23. Springer, 2009. 25, 26

[PS10] Bijan Parsia and Thomas Schneider. The modular structure of an ontology: An empirical study. In Proceedings of the 12 ${ }^{\text {th }}$ International Conference on Principles of Knowledge Representation and Reasoning (KR 2010), Toronto, Ontario, Canada, May 9-13, 2010. AAAI Press, 2010. 33

[PWCF15] Pavlos Peppas, Mary-Anne Williams, Samir Chopra, and Norman Y. Foo. Relevance in belief revision. Artificial Intelligence, 229:126-138, 2015. 25

[QLB06] Guilin Qi, Weiru Liu, and David Bell. A revision-based approach to handling inconsistency in description logics. Artificial Intelligence Review, 26(1-2):115-128, 2006. 3

[QP07] Guilin Qi and Jeff Z. Pan. A stratification-based approach for inconsistency handling in description logics. In Proceedings of the International Workshop on Ontology Dynamics (IWOD 2007), Innsbruck, Austria, June 7, 2007, volume 8396, 2007. 103, 104, 106

[RCH12] Ana Armas Romero, Bernardo Cuenca Grau, and Ian Horrocks. More: Modular combination of OWL reasoners for ontology classification. In Proceedings of the $11^{\text {th }}$ International Semantic Web Conference (ISWC 2012), Boston, MA, USA, November 11-15, 2012, volume 7649 of Lecture Notes in Computer Science, pages 1-16. Springer, 2012. 26

[Rei87] Raymond Reiter. A theory of diagnosis from first principles. Artificial Intelligence, 32(1):57-95, 1987. 20, 121, 122

[Rib13] Márcio Moretto Ribeiro. Belief Revision in Non-Classical Logics. Springer Briefs in Computer Science. Springer, 2013. 3, 10, 11, 12, 13, 14, 15, 16, 17, 18, 19, 20, 121

[RKCH16] Ana Armas Romero, Mark Kaminski, Bernardo Cuenca Grau, and Ian Horrocks. Module extraction in expressive ontology languages via datalog reasoning. Journal of Artificial Intelligence Research, 55:499-564, 2016. 27, 29, 123 
[Rot92] Hans Rott. Preferential belief change using generalized epistemic entrenchment. Journal of Logic, Language and Information, 1(1):45-78, 1992. 103

[RRW14] Fillipe Resina, Márcio Moretto Ribeiro, and Renata Wassermann. Algorithms for multiple contraction and an application to OWL ontologies. In Proceedings of the $3^{\text {rd }}$ Brazilian Conference on Intelligent Systems (BRACIS 2014), São Paulo, Brazil, October 18-22, 2014, pages 366-371. IEEE Computer Society, 2014. 22, 37

[RW09] Márcio Moretto Ribeiro and Renata Wassermann. Base revision for ontology debugging. Journal of Logic and Computation, 19(5):721-743, 2009. 10, 11

[SC03] Stefan Schlobach and Ronald Cornet. Non-standard reasoning services for the debugging of description logic terminologies. In Proceedings of the $18^{\text {th }}$ International Joint Conference on Artificial Intelligence (IJCAI 2003), Acapulco, Mexico, August 9-15, 2003, pages 355-362. Morgan Kaufmann, 2003. 12, 19, 37, 53

[Sch05] Stefan Schlobach. Debugging and semantic clarification by pinpointing. In Proceedings of the $2^{\text {nd }}$ European Semantic Web Conference (ESWC 2005), Heraklion, Crete, Greece, May 29 - June 1, 2005, volume 3532 of Lecture Notes in Computer Science, pages 226-240. Springer, 2005. 37

[Sco92] David W. Scott. Multivariate Density Estimation: Theory, Practice, and Visualization. John Wiley \& Sons Inc, 1992. 116, 117

[SMRW18] Yuri David Santos, Vinícius Bitencourt Matos, Márcio Moretto Ribeiro, and Renata Wassermann. Partial meet pseudo-contractions. International Journal of Approximate Reasoning, 103:11-27, December 2018. 26, 123

[SQJH08] Boontawee Suntisrivaraporn, Guilin Qi, Qiu Ji, and Peter Haase. A modularizationbased approach to finding all justifications for OWL DL entailments. In Proceedings of the $3^{\text {rd }}$ Asian Semantic Web Conference (ASWC 2008), Bangkok, Thailand, December 8-11, 2008, volume 5367 of Lecture Notes in Computer Science, pages 1-15. Springer, 2008. 4, 19, 20, 26, 27, 28, 35, 37, 38, 39, 53, 60, 61, 88, 121, 122

[SSZ09] Ulrike Sattler, Thomas Schneider, and Michael Zakharyaschev. Which kind of module should I extract? In Proceedings of the 22 ${ }^{\text {nd }}$ International Workshop on Description Logics (DL 2009), Oxford, UK, July 27-30, 2009, volume 477 of CEUR Workshop Proceedings. CEUR-WS.org, 2009. 28, 29, 30, 31, 32, 88

[Sun08] Boontawee Suntisrivaraporn. Module Extraction and Incremental Classification: A Pragmatic Approach for $\mathcal{E}^{+}$Ontologies. In Lecture Notes in Computer Science, pages 230-244. Springer Berlin Heidelberg, 2008. 26

[Sun09] Boontawee Suntisrivaraporn. Polynomial time reasoning support for design and maintenance of large-scale biomedical ontologies. PhD thesis, Dresden University of Technology, 2009. 26

[TP12] Dmitry Tsarkov and Ignazio Palmisano. Chainsaw: a metareasoner for large ontologies. In Proceedings of the $1^{\text {st }}$ International Workshop on OWL Reasoner Evaluation (ORE2012), Manchester, UK, July 1 ${ }^{\text {st }}$, 2012, volume 858 of CEUR Workshop Proceedings. CEUR-WS.org, 2012. 26

[Tsa12] Dmitry Tsarkov. Improved algorithms for module extraction and atomic decomposition. In Proceedings of the $25^{\text {th }}$ International Workshop on Description Logics (DL 2012), Rome, Italy, June 7-10, 2012, volume 846 of CEUR Workshop Proceedings. CEUR-WS.org, 2012. 27, 29, 34 
[Was00] Renata Wassermann. An algorithm for belief revision. In Proceedings of the rth International Conference on Principles of Knowledge Representation and Reasoning (KR 2000), Breckenridge, Colorado, USA, April 11-15, 2000, pages 345-352. Morgan Kaufmann, 2000. 11, 20

[Was01] Renata Wassermann. On structured belief bases. In Frontiers in Belief Revision, pages 349-367. Springer Netherlands, Dordrecht, 2001. 108

[Wil94] Mary-Anne Williams. On the logic of theory base change. In Proceedings of the $4^{\text {th }}$ European Workshop on Logics in Artificial Intelligence, (JELIA 1994), York, UK, September 5-8, 1994, volume 838 of Lecture Notes in Computer Science, pages 86-105. Springer, 1994. 103

[YJW14] Y. Yuan, S. Ju, and X. Wen. Evaluative multiple revision based on core beliefs. Journal of Logic and Computation, 25(3):781-804, sep 2014. 13 